\title{
Cerebrovascular Dysfunction and Degeneration in Alzheimer's Disease Pathophysiology
}

Dominic Dimitri Quintana

West Virginia University, ddquintana@mix.wvu.edu

Follow this and additional works at: https://researchrepository.wvu.edu/etd

Part of the Molecular and Cellular Neuroscience Commons, and the Nervous System Diseases

\section{Commons}

\section{Recommended Citation}

Quintana, Dominic Dimitri, "Cerebrovascular Dysfunction and Degeneration in Alzheimer's Disease Pathophysiology" (2020). Graduate Theses, Dissertations, and Problem Reports. 7941.

https://researchrepository.wvu.edu/etd/7941

This Dissertation is protected by copyright and/or related rights. It has been brought to you by the The Research Repository @ WVU with permission from the rights-holder(s). You are free to use this Dissertation in any way that is permitted by the copyright and related rights legislation that applies to your use. For other uses you must obtain permission from the rights-holder(s) directly, unless additional rights are indicated by a Creative Commons license in the record and/ or on the work itself. This Dissertation has been accepted for inclusion in WVU Graduate Theses, Dissertations, and Problem Reports collection by an authorized administrator of The Research Repository @ WVU.

For more information, please contact researchrepository@mail.wvu.edu. 
Graduate Theses, Dissertations, and Problem Reports

2020

Cerebrovascular Dysfunction and Degeneration in Alzheimer's

Disease Pathophysiology

Dominic Dimitri Quintana

Follow this and additional works at: https://researchrepository.wvu.edu/etd

Part of the Molecular and Cellular Neuroscience Commons, and the Nervous System Diseases

Commons 


\title{
Cerebrovascular Dysfunction and Degeneration in Alzheimer's Disease Pathophysiology
}

\author{
Dominic D. Quintana
}

\author{
Dissertation submitted \\ to the School of Medicine \\ at West Virginia University
}

in partial fulfillment of the requirements for the degree of

\section{Doctor of Philosophy in}

Neuroscience

\author{
James W. Simpkins, Ph.D., Chair \\ Lisa M. Salati, Ph.D., Vice Pres. of Grad. Edu. \\ Candice M. Brown, Ph.D. \\ Werner J. Geldenhuys, Ph.D. \\ Paul R. Lockman, Ph.D. \\ Elizabeth B. Engler-Chiurazzi, Ph.D.
}

Department of Neuroscience

Morgantown, West Virginia

2020

Keywords: Alzheimer's Disease, Cerebrovasculature, Cerebral Amyloid Angiopathy, Hypoperfusion, Endothelial Cells, Mitochondrial Dysfunction 


\title{
ABSTRACT
}

\section{Cerebrovascular Dysfunction and Degeneration in Alzheimer's Disease Pathophysiology}

\author{
Dominic D. Quintana
}

Alzheimer's disease (AD) is a terminal illness and the most common form of dementia, which disproportionately affects the aged population. The pathophysiology of $\mathrm{AD}$ is characterized by neurodegeneration that slowly progresses, affecting regions of the brain that are involved in learning, memory, language, and executive function. In patients with the disease, early symptoms include non-disruptive forgetfulness that evolves into the inability to form new memories and ultimately the loss of autonomy at late stages. Histopathological hallmarks in the brain from patients with $\mathrm{AD}$ is the presence of amyloid- $\beta$ (A $\beta$ )-plaques and neurofibrillary tangles (NFT) deposited in the parenchyma. Since the discovery of these hallmarks, the majority of $\mathrm{AD}$ research has disproportionately focused on $\mathrm{A} \beta$-plaques and NFT. Although the etiology of AD remains unknown, considerable advances have been made describing the cellular, molecular, and genetic contributions to the disease. Aging is the important risk factor for the development of $\mathrm{AD}$, many other factors that increase the risk of developing AD later in life are vascular in nature. The function of the cardiovascular system is known to decline during healthy aging, and the same is true for the cerebrovasculature. Empirical evidence has demonstrated a decline cerebrovascular function in AD that exceeds the decline that occurs in healthy aging. Cerebrovascular dysfunction is the major contributor to the development of hypoperfusion and hypometabolism in patients diagnosed with AD. Cerebral amyloid angiopathy (CAA) is a neuropathological condition defined by the abnormal accumulation of $A \beta$ on the walls of the cerebrovasculature. CAA occurs in as many as $90 \%$ of patients with $\mathrm{AD}$ and is implicated in the weakening of the walls of cerebral blood vessels. The occurrence of microhemorrhages, aneurysms, and microinfarctions are pathological manifestations associated with weakened walls of cerebral blood vessels in the brains of patients with confirmed AD. Noteworthy, cerebrovascular dysfunction, hypoperfusion, and hypometabolism occur before the onset of A $\beta$-plaque and NFT deposition in the brain of patients and animal models with AD. These findings provide a compelling basis that suggest a prominent role of dysfunctional cerebrovasculature in the etiology and for the progression of AD.

Although the overwhelming evidence that implicates cerebrovascular dysfunction in $\mathrm{AD}$, a thorough account of the changes that occur to the cerebrovasculature nor the mechanisms that drive these changes during the development and progression of AD has not been previously reported. The overarching goal(s) of this work are to; (1) provide a thorough 
description of the changes that occur to the cerebrovasculature during age and the progression of $\mathrm{AD}$; (2) describe the mechanisms involved in cerebrovascular damage in AD; and (3) characterize the degeneration that results from cerebrovascular hypoperfusion. These overarching goals were achieved by completing five separate studies. Described in study 1 , we investigated the effects of hypoxia on astrocytic mitochondria by assessing mitochondrial fission-fusion dynamics, reactive oxygen species production, synthesis of ATP, and mitophagy. Overall, we found a drastic mitochondrial network change that is triggered by metabolic crisis during hypoxia; these changes are followed by mitochondrial degradation and retraction of astrocytic extensions during reoxygenation. In study 2 , we provide a novel model for the gradual development of cerebrovascular hypoperfusion in mice. Cerebrovascular hypoperfusion developed over 34-days by inserting an ameroid constrictor ring and microcoil bilaterally around the external carotid arteries. We investigated the neurodegenerative effects of hypoperfusion in mice by assessing both gray and white matter pathology. Histopathological analyses of the brain revealed neuronal and axonal degeneration as well as necrotic lesions. The most severely affected regions were located in the hippocampus and corpus callosum. Described in study 3, we performed a series of experiments to investigate the effects of $A \beta$ on cerebrovascular endothelial cells. In this study, we focused on characterizing the changes to mitochondrial oxidative phosphorylation, superoxide production, mitochondrial calcium, ATP synthesis, and endothelial cell death. These results describe a mechanism for mitochondrial degeneration caused by the production of mitochondrial superoxide, which was driven by increased mitochondrial $\mathrm{Ca}^{2+}$ uptake. We found that persistent superoxide production injures mitochondria and disrupts electron transport in cerebrovascular endothelial cells. In study 4, we developed a method to evaluate the cerebrovasculature of the whole-brain and constructed analyses to assess the angioarchitecture. We used vascular corrosion casting method to replicate the cerebrovasculature in adult mice and used MicroCT to acquire volumetric imaging data of the cerebrovascular network at a resolution required to investigate the microvasculature. Our analyses of the cerebrovasculature evaluated the morphology, topology, and organization of the angioarchitecture. With these developments, we investigated the effects of age and progression of disease on the cerebrovasculature in wild type mice and the triple transgenic mouse model of AD. Study 5 provides data describing degenerative changes to the microvascular network that progress with age in the triple transgenic mouse model of AD. These changes to the microvasculature occurred early, before the onset of A $\beta$-plaque deposition and NFT development.

Overall, this body of work provides evidence of an early cerebrovascular disruption in the etiology of $\mathrm{AD}$ that progresses with age. $\mathrm{A} \beta$ mediates early cerebrovascular damage through direct interaction with vascular endothelial cells. Microvascular degeneration can lead to hypoperfusion which damages both gray and white matter. Hypoperfusion-associated hypoxia may mediate parenchymal damage by disrupting mitochondrial fission-fusion dynamics and enhancing mitophagy. These data provide a basis for the development of novel therapeutic strategies that target the changes to the cerebrovasculature for the treatment of AD. These observations may substantiate a prophylactic strategy for the treatment of AD by preventing the initial factors that lead to compromised cerebrovasculature. 
This dissertation is dedicated to my parents and two brothers

for their endless love, support and encouragement 


\section{ACKNOWLEDGEMENTS}

I would like to thank Dr. James W. Simpkins for his excellent mentorship and guidance. Through your expert instruction and guidance, you have provided me with the knowledge and wisdom that will help me become the best research scientist that I can be.

I would like to thank my committee members, Dr. Candice M. Brown, Dr. Elizabeth B. EnglerChiurazzi, Dr. Lisa M. Salati, Dr. Werner J. Geldenhuys, and Dr. Paul R. Lockman for their guidance and insight, which has helped me navigate through this project.

I would also like to thank past and current members of the Simpkins laboratory; Dr. Saumyendra N. Sarkar for his guidance and many discussions of the literature; Dr. Elizabeth B. Engler-Chiurazzi for her expertise in statistical analyses; Dr. Xuefang Ren and Dr. Heng $\mathrm{Hu}$ for their efforts through the Stroke Core Facility to perform many experimental surgeries used in the herein project; Dr. Stephanie Rellick for her assistance with running several biochemical assays; and Deborah R. Corbin for her assistance with experiments and maintaining our mouse colony.

A special thanks go to Yamini Anantula, Jorge A. Garcia, and Sara E. Lewis for their considerable assistance with performing experiments.

I would also like to thank Dr. Ashley E. Russell, Dr. John Z. Cavendish, Keyana N. Porter, and Dr. Sujung Jun for their support and friendship. 


\section{Table of Contents}

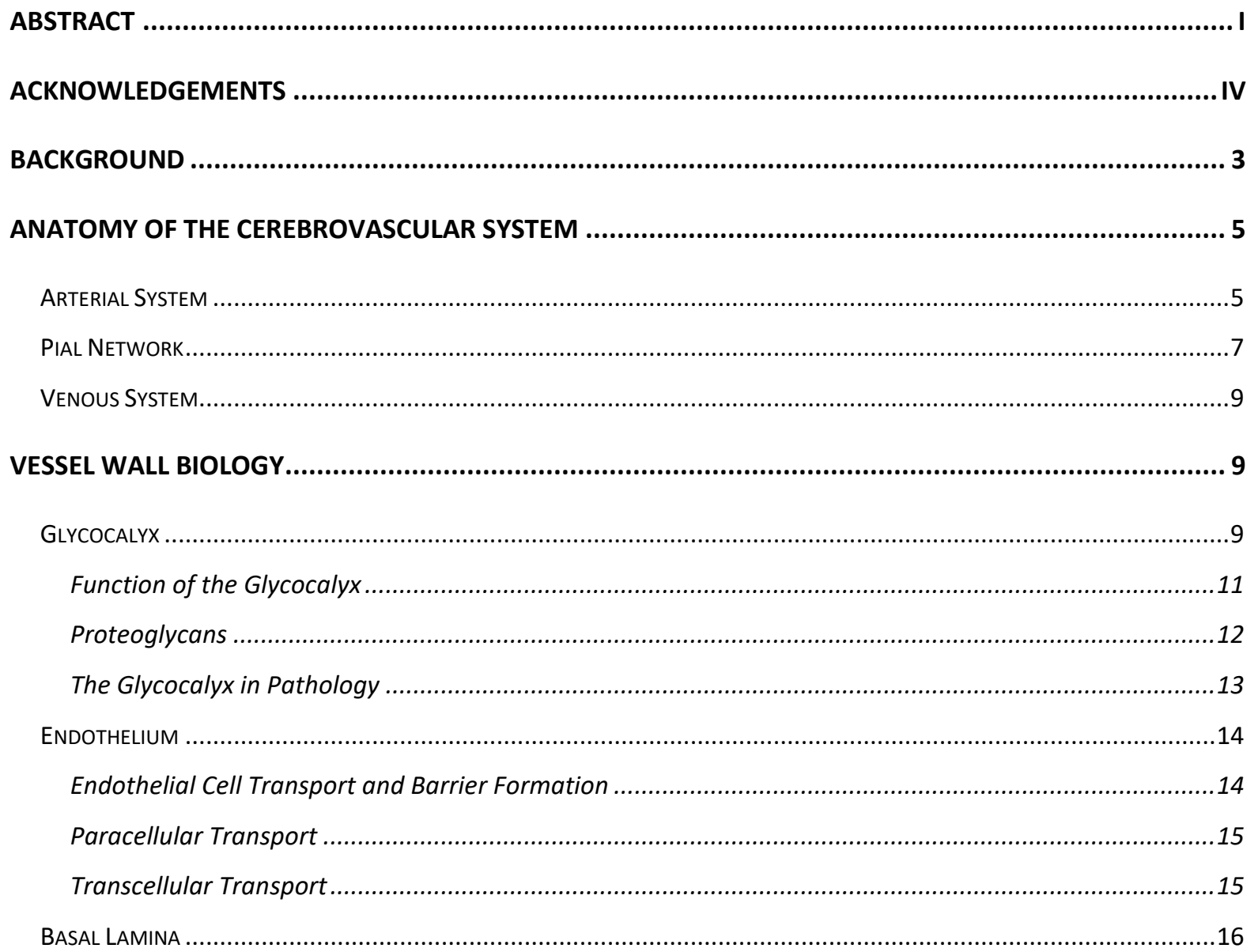

\section{CELLULAR AND MOLECULAR BIOLOGY OF THE CEREBROVASCULAR SYSTEM .................................... 19}

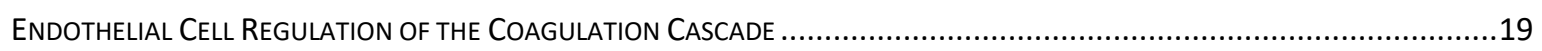

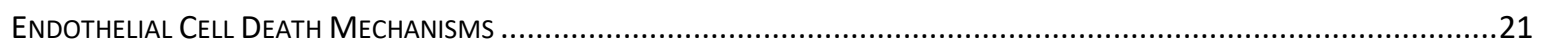

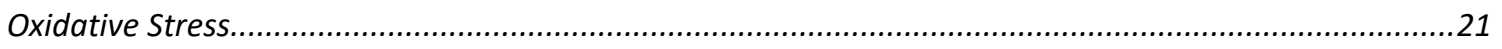

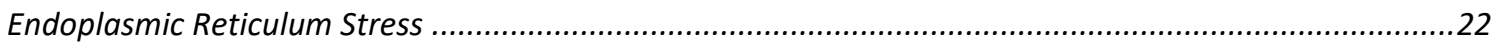

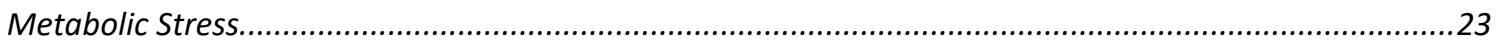

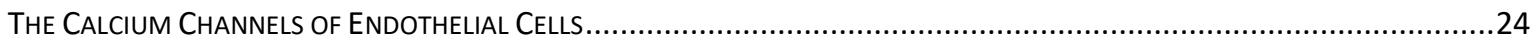

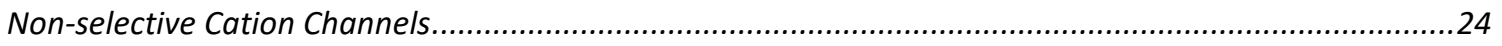

HEMODYNAMICS AND HEMORHEOLOGY OF THE VASCULAR HIERARCHY ........................................ 25

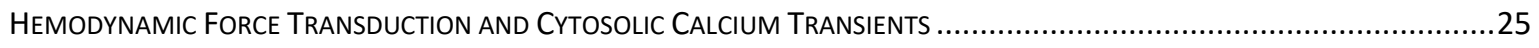

Hemodynamic Forces Regulate AngIOgenesis ANd VASCUlar MaINTENANCE Signaling .......................................26

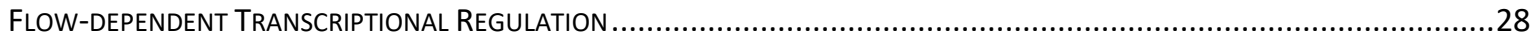




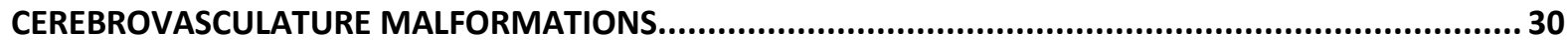

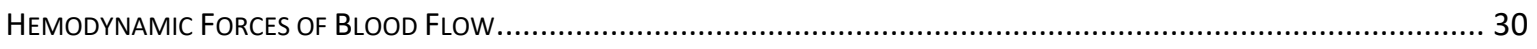

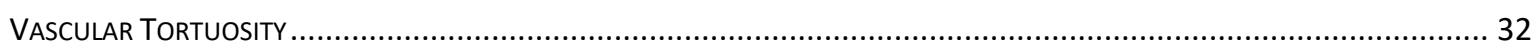

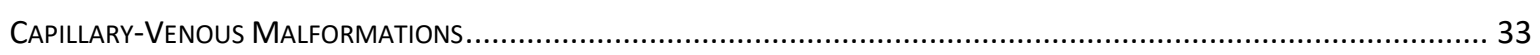

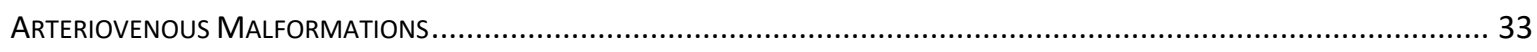

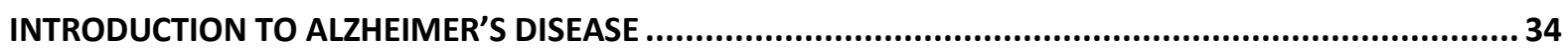

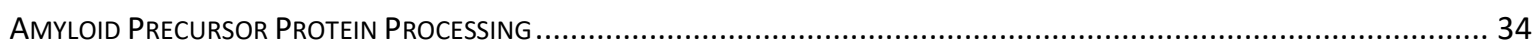

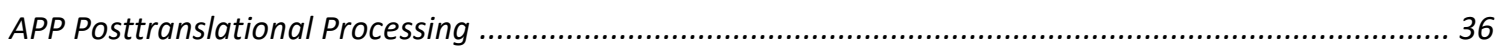

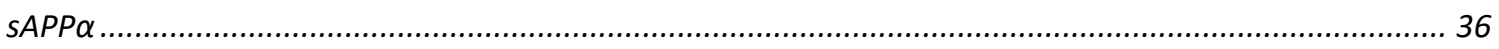

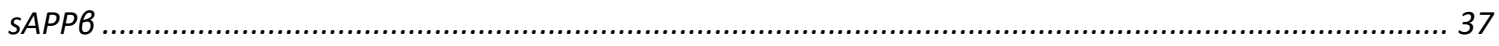

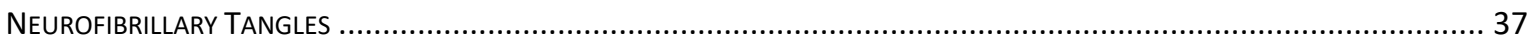

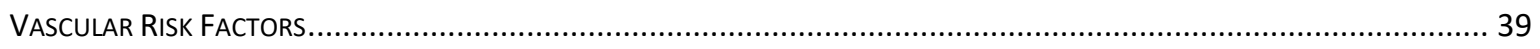

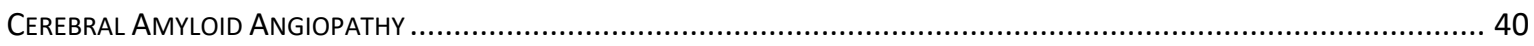

ALIGNMENT OF STUDY AIMS WITH PROJECT GOALS......................................................................... 42

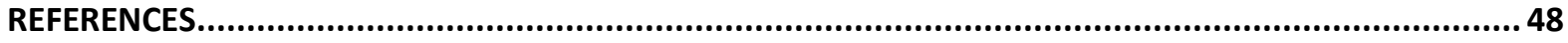

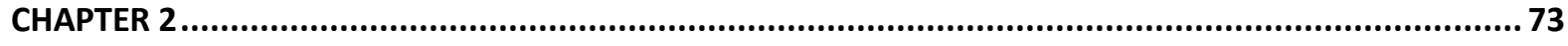

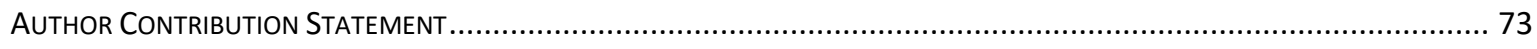

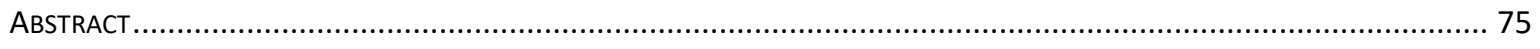

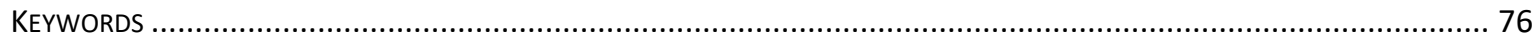

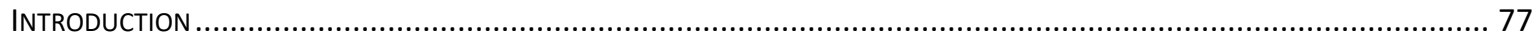

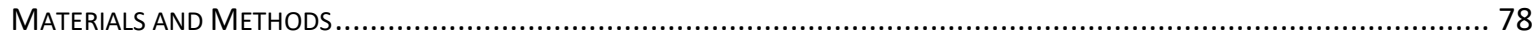

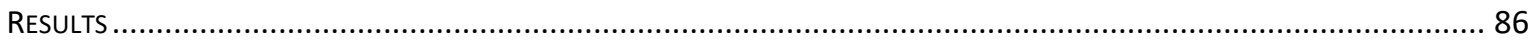

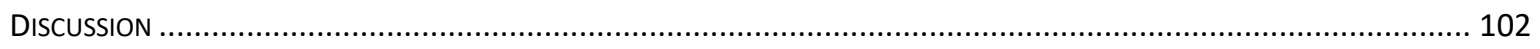

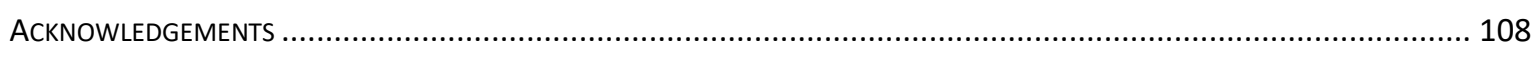

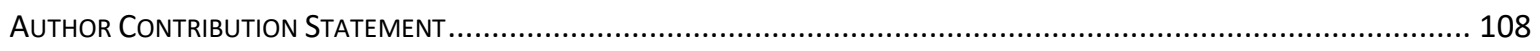

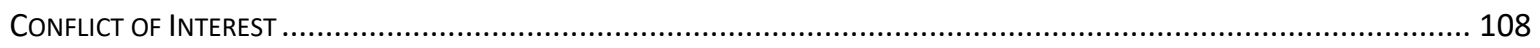

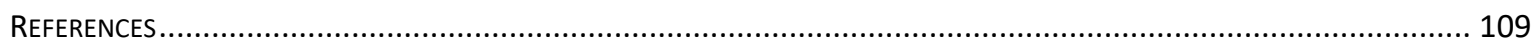

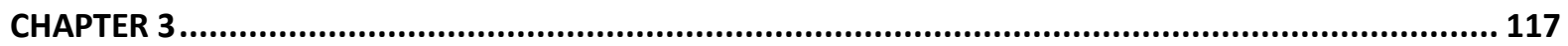

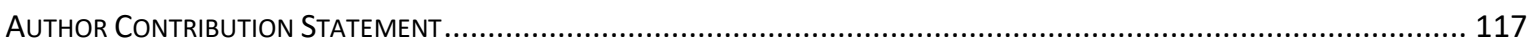

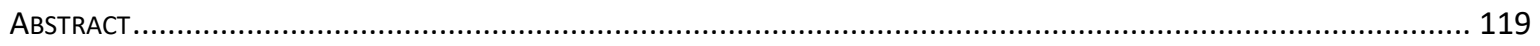

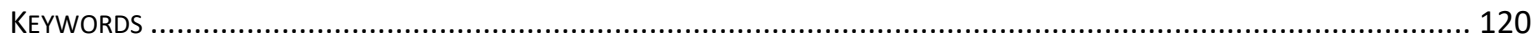




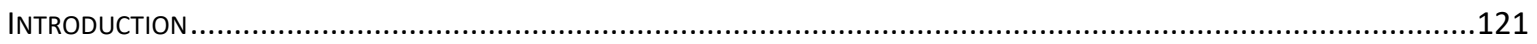

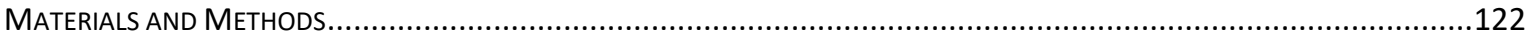

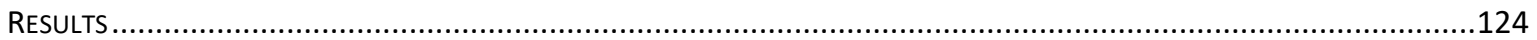

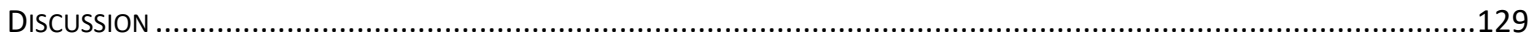

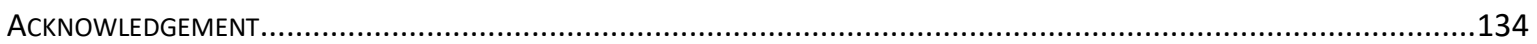

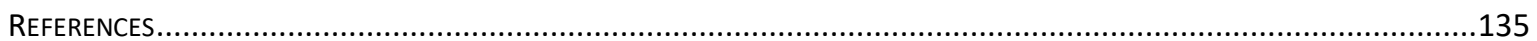

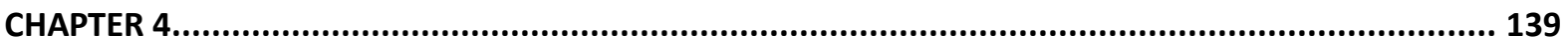

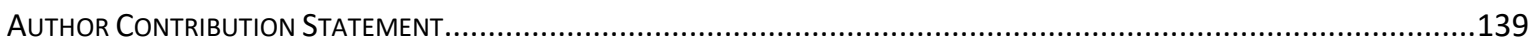

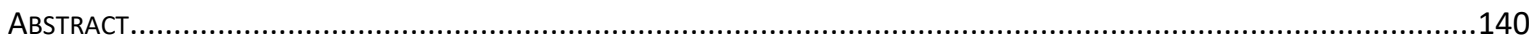

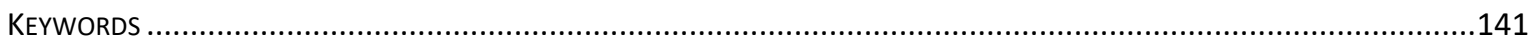

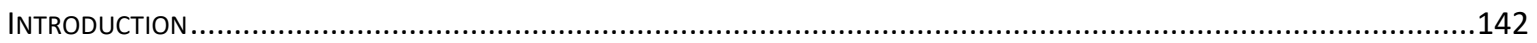

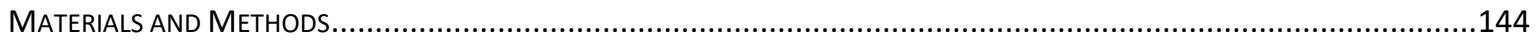

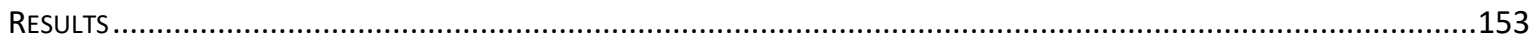

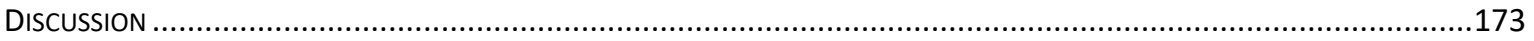

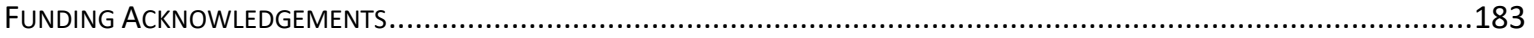

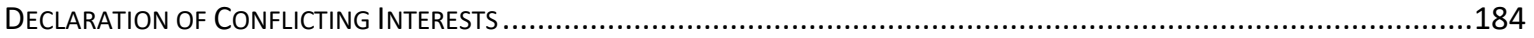

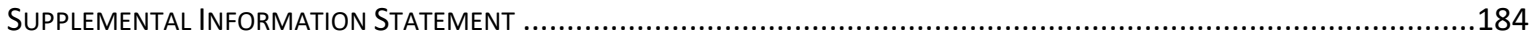

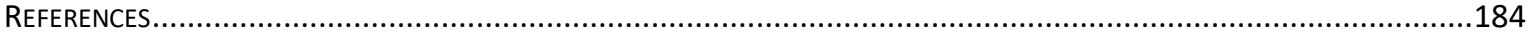

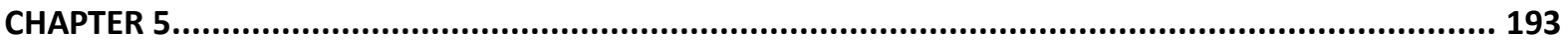

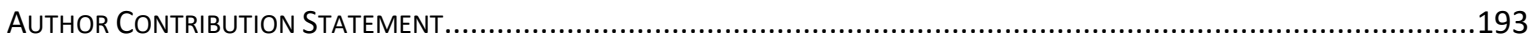

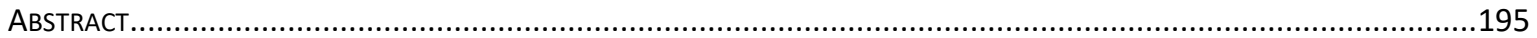

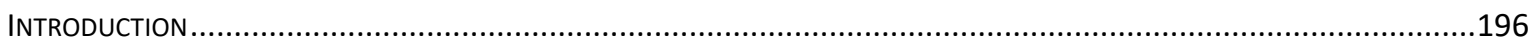

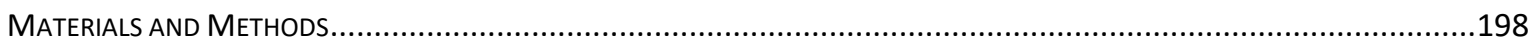

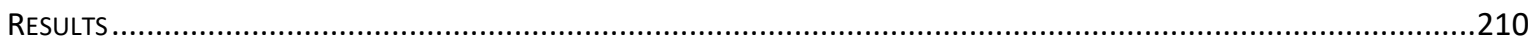

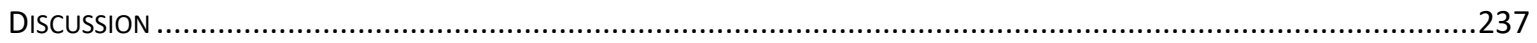

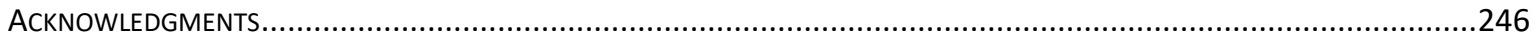

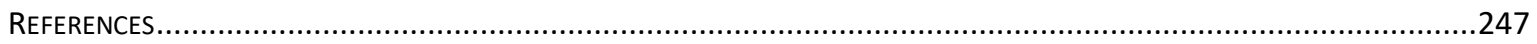

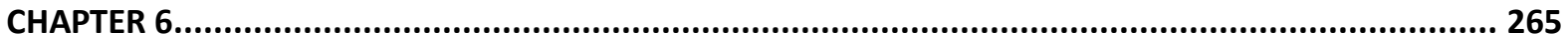

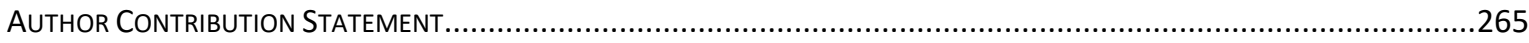

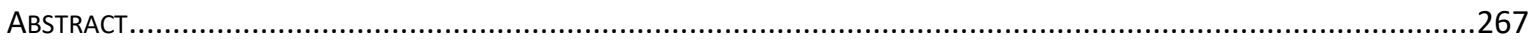

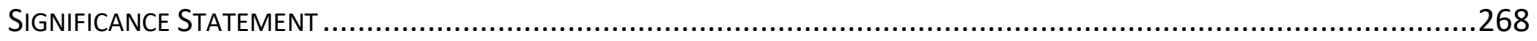

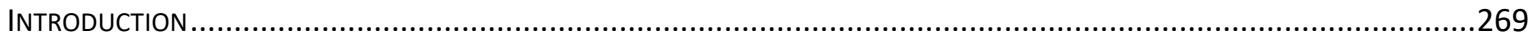

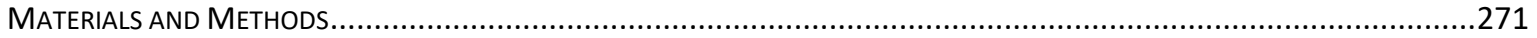


RESULTS

DISCUSSION

REFERENCES. 306

\section{Publications}

Quintana, D.D., Garcia, J.A., Anantula, Y., Rellick, S.L., Engler-Chiurazzi, E.B., Sarkar, S.N., Brown, C.M., Simpkins, J.W., 2020. Amyloid- $\beta$ Causes Mitochondrial Dysfunction via a Ca2+-Driven Upregulation of Oxidative Phosphorylation and Superoxide Production in Cerebrovascular Endothelial Cells. J. Alzheimers. Dis. 75, 119-138. https://doi.org/10.3233/JAD-190964

Quintana, D.D., Garcia, J.A., Sarkar, S.N., Jun, S., Engler-Chiurazzi, E.B., Russell, A.E., Cavendish, J.Z., Simpkins, J.W., 2018a. Hypoxia-reoxygenation of primary astrocytes results in a redistribution of mitochondrial size and mitophagy. Mitochondrion. https://doi.org/10.1016/J.MITO.2018.12.004

Quintana, D.D., Lewis, S.E., Anantula, Y., Garcia, J.A., Sarkar, S.N., Cavendish, J.Z., Brown, C.M., Simpkins, J.W., 2019. The cerebral angiome: High resolution MicroCT imaging of the whole brain cerebrovasculature in female and male mice. Neuroimage. https://doi.org/10.1016/j.neuroimage.2019.116109

Quintana, D.D., Ren, X., Hu, H., Engler-Chiurazzi, E.B., Rellick, S.L., Lewis, S.E., Povroznik, J.M., Simpkins, J.W., Alvi, M., 2018b. Gradual common carotid artery occlusion as a novel model for cerebrovascular Hypoperfusion. Metab. Brain Dis. 33, 2039-2044. https://doi.org/10.1007/s11011-018-0312-5 


\section{Chapter 1}

\section{Introduction and Literature Review}




\section{Introduction and Literature Review}

Ageing is a terminal condition, characterized by a slow progressive loss of an organism's viable state. The complex network of factors that determine an individual's unique pattern of aging is dependent of the genetic, environmental, and circumstantial elements that occurs throughout life. Aging is unavoidable. However, the pathway that we take during aging can be refined with technological and biomedical advancement, so that our course through life is a pleasant one.

\section{Background}

The cerebrovascular system is a complex array of interconnected pathways that participate in a stringently regulated transport of fluid throughout an elaborate network of vessels. These systems provide crucial functions that maintain metabolic and homeostatic states of the cerebral tissue. Under physiological conditions, the hemodynamic demand of the brain fluctuates in accordance to the specific condition and activity of the cerebral tissue. Frequent changes in neuronal activity during normal function determine specific hemodynamic parameters to be met at the submicron level. Therefore, to match these functional requirements, the cerebrovasculature must maintain a highly dynamic and sensitive state to remain sufficiently responsive to the fluctuating demand of the central nervous tissue. Due to this highly sensitive nature, the cerebrovascular system is exceptionally responsive to pathological conditions. It is likely that even the smallest dysregulation of physiological cerebrovascular signaling or function will manifest into vascular dysfunction implicating the dynamic 
properties of the system and would compromise its regulatory potential and likely result in a failure to meet hemodynamic demand of the cerebral tissue.

Vascular pathology is a known risk factor for the development of Alzheimer's disease (AD) and other dementias. However, the precise role of dysfunctional vasculature in $\mathrm{AD}$ progression remains unknown. The aim of this research is to identify the mechanism by which the accumulation of amyloid- $\beta(\mathrm{A} \beta)$ on or within the cerebrovasculature exerts structural and functional changes associated with vascular damage and degeneration, and to determine how these changes influence the progression of $\mathrm{AD}$.

A role for the dysfunction of the cerebrovasculature in the development and progression of $\mathrm{AD}$ is likely to occur. A number of reports made in human $\mathrm{AD}$ show regional hypoperfusion, hypometabolism, and blood-brain barrier hyperpermeability; the hallmark pathology of dysfunctional vasculature (de la Torre 2004; Brundel et al. 2012b; Brundel et al. 2012a). To date, a large prevalence of patients with $A D$ are at risk for developing severe vascular conditions including, hemorrhagic stroke, spontaneous cerebral emboli, cerebral microinfarctions, and microhemorrhages (Chi et al. 2013; Brundel et al. 2012a; Purandare and Burns 2009; Tolppanen et al. 2013). The increased prevalence of developing these severe vascular conditions is substantial evidence indicating a degrading cerebrovascular integrity in patients with AD. Recently, a body of evidence has emerged documenting cerebrovascular dysfunction preceding cognitive decline in AD patients (de la Torre 2010; Jellinger 2010; Kalaria 2010). Although, vascular issues are co-morbid in $90 \%$ of all AD cases, a significant deficit exists in the field that adequately describes the role for the cerebrovascular system in the neurodegeneration in $\mathrm{AD}$. 
The entirety of this research has been conducted to assess the following hypothesis; the accumulation of $A \beta$ on the cerebral microvasculature compromises capillary function resulting in cerebrovascular hypoperfusion and vascular degeneration.

The hypothesis will be assessed by means of completing the following research aims: (1) to identify the specific structural and functional changes that occur to the cerebral vasculature during the progression of $\mathrm{AD}$; (2) to characterize the time-course of cerebral amyloid angiopathy (CAA) development in 3xTg AD mice and to evaluate its pathogenicity on cerebral microcirculation; and (3) to assess the hemodynamic state that follows the changes to the cerebrovasculature in aging $3 \times \mathrm{Tg} \mathrm{AD}$ mice.

\section{Anatomy of the Cerebrovascular System}

\section{Arterial System}

Arterial blood supply can enter the cranium through two pairs of cerebral arteries, the carotid arteries and the vertebral arteries (Figure 1.1). The vertebral arteries supply blood to the upper part of the spinal cord, brain stem, cerebellum and posterior part of the brain. The vertebral arteries lie on the surface of the brain stem and join at the medulla oblongata, forming the basilar artery. Projecting laterally from the vertebral arteries over the surface of the brain stem, are the posterior inferior cerebellar arteries. Further along the vertebral arteries, the anterior spinal arteries project toward the midline, fusing into a single anterior spinal artery over the medulla oblongata. The basilar artery that is formed by the joining of the two vertebral arteries ascends superiorly over the basilar sulcus ventral to the pons, where it then terminates by branching into the two posterior cerebral arteries at the Ponto mesencephalic junction close 
to the pituitary stalk. The basilar artery projects a number of branches laterally, including the anterior inferior cerebellar arteries, labyrinthine arteries, pontine arteries, and superior cerebellar arteries. The posterior cerebral arteries supply blood to the posterior aspect of the brain, are formed from the branching of the basilar artery. The posterior cerebral arteries project a number of branches into the cortex, including the anterior temporal branching that is distributed between the uncus and the anterior part of the fusiform gyrus. The posterior temporal branching of the posterior cerebral arteries extends into the fusiform and the inferior temporal gyri. The lateral occipital branching of the posterior cerebral artery further branch into the anterior, middle, and posterior inferior temporal arteries. The medial occipital branch extends into the calcarine, cuneus, gyrus lingualis, and the posterior aspect of the occipital lobe. A stroke involving the posterior cerebral arteries, can cause the development of prosopagnosia, defects in the oculomotor nerve, defects of the facial nerve, Horner's syndrome and visual field defects. The posterior cerebral arteries communicate blood flow to the middle cerebral arteries via posterior communicating arteries. The middle cerebral arteries project laterally to the sulcus then branching to the lateral cerebral cortex, temporal lodes, and insular cortices. Following a stroke that involved the middle cerebral arteries, paralysis, sensory loss, Broca's aphasia, Wernicke's aphasia, and contralateral neglect syndrome can result. Converging on the middle cerebral arteries, the carotid arteries constitute the second route of arterial blood entering the cranium. Branching from the middle cerebral arteries, the anterior cerebral arteries extend frontally then loop back around between the two hemispheres at the midline fissure above the corpus callosum and continue to extend posteriorly. The anterior cerebral arteries supply blood to the frontal lobes and to the superior medial parietal lobes. Anterior cerebral artery syndrome can result following a stroke that involved the anterior 
cerebral arteries. Anterior cerebral artery syndrome is characterized by the loss of sensory perception and weakness to the foot and lower leg contralateral to the lesion. The anterior cerebral arteries are joined by a conduit noted as the anterior communicating artery. Together, the posterior cerebral arteries, posterior communicating arteries, internal carotid arteries, anterior cerebral arteries, and the anterior communicating artery composes an arterial system known as the circle of Willis. The circle of Willis is a ring-like structure formed by anastomoses that serves a function of redundancy allowing collateral circulation of cerebral blood flow. This redundancy is advantageous in the event of an occlusion of any artery that composes the circle, allowing the delivery of blood from the remaining anastomotic segments.

\section{Pial Network}

Consisting of large veins and arteries, the pial network forms a large number of loops within the venous and arterial networks. These loops serve vital functions in the maintenance of continuous blood flow by providing redundant pathways of blood delivery to cortical areas that protect cerebral tissue against ischemic perturbations in blood flow (Blinder et al. 2010). In a mathematical simulation of stroke, the occlusion of any pial vessel is compensated for by the shunting of blood flow from other areas to the occluded region (Schaffer et al. 2006). In contrast to these vascular loops providing redundancy of blood flow, arteriovenous anastomoses are another form of vascular loop that seem to contribute to instead of protecting against pathology (Duvernoy et al. 1981). The development of arteriovenous anastomoses is problematic in that these new networks provide pathways that allow blood flow to bypass capillary networks and flow directly into the venous system. 


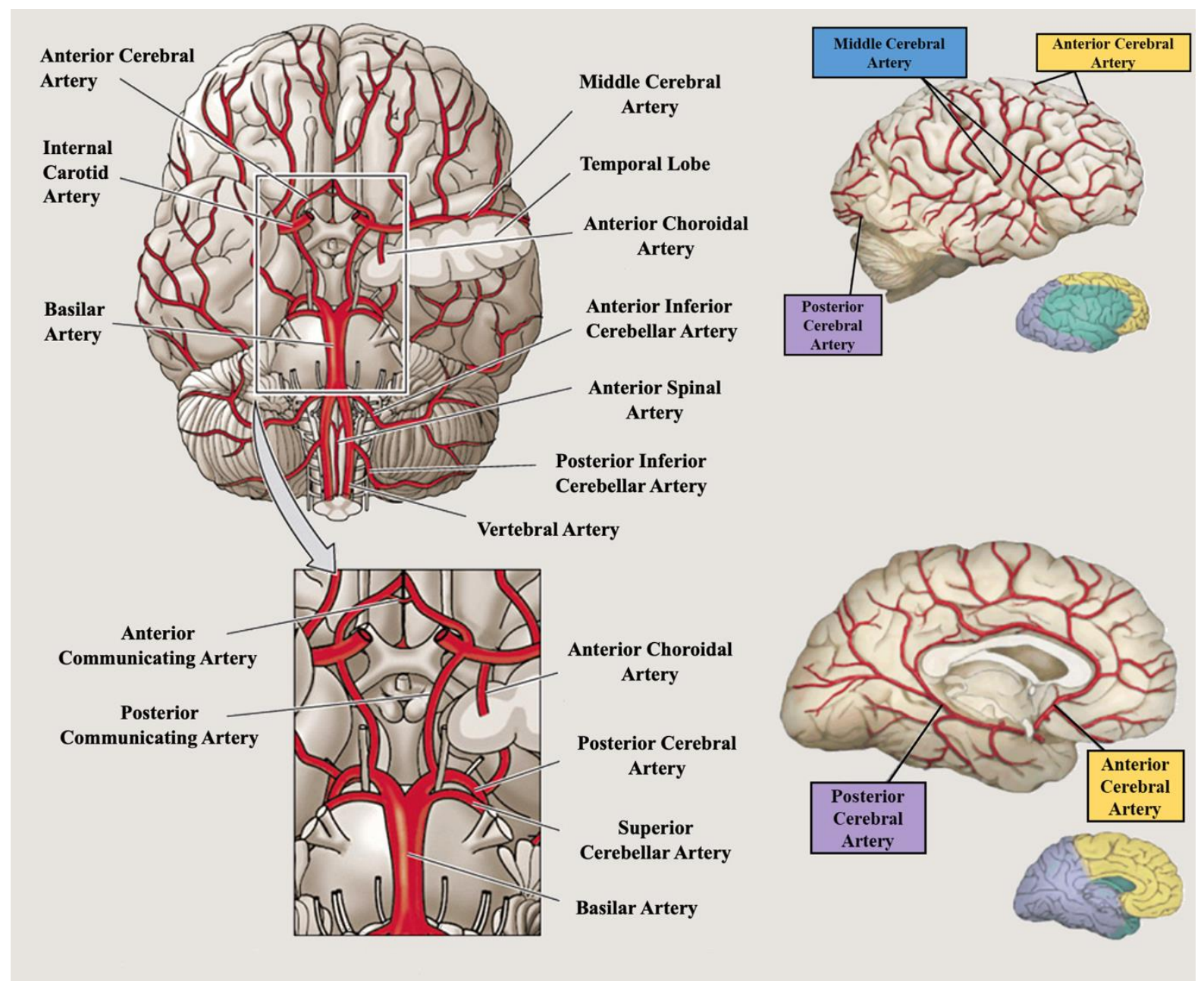

Figure 1.1. Diagram of the major cerebral arteries. Ventral perspective of the major cerebral arteries including the vertebral arteries, internal carotid arteries. Magnified ventral perspective depicting the major cerebral arteries that form the circle of Willis. Sagittal perspective of the anterior cerebral arteries, middle cerebral arteries, and posterior cerebral arteries on the surface of the brain. Sagittal perspective at the midline of the brain depicting the anterior cerebral artery and posterior cerebral artery within the internal structures of a hemisphere of the brain. 


\section{Venous System}

The organization of the venous system is inverse of the arterial system in that many venioles systematically join into larger veins and these veins feed blood to the sinus. The cerebral venous system often classifies into five divisions, the external and internal veins, the meningeal veins, dural sinuses, and the posterior fossa veins (Kiliç and Akakin 2008). The cerebral veins are often divided into internal and external veins that correlates to the area of the brain that they drain. The external venous system consists of the cortical veins and the sagittal sinus that drains the superficial surface of the cerebral hemispheres (Figure 1.2, top). The internal venous system consists of the straight sinus, sigmoid sinus, and the transverse sinus including the deeper cortical veins (Figure 1.2, bottom). Of the external division, consisting of the superior, inferior, and middle cerebral veins, which drain into the basal vein (Kiliç and Akakin 2008).

\section{Vessel Wall Biology}

\section{Glycocalyx}

The glycocalyx is the innermost lumenal layer of the vascular wall extending from the lumen to the internal elastic lamina. The glycocalyx is a carbohydrate-rich matrix that lines the surface of endothelial cells and is thought to maintain this position via interactions of proteoglycans and glycoproteins, which form a structural backbone that facilitate the incorporation of other molecules from blood plasma and endothelial derived. The glycocalyx 

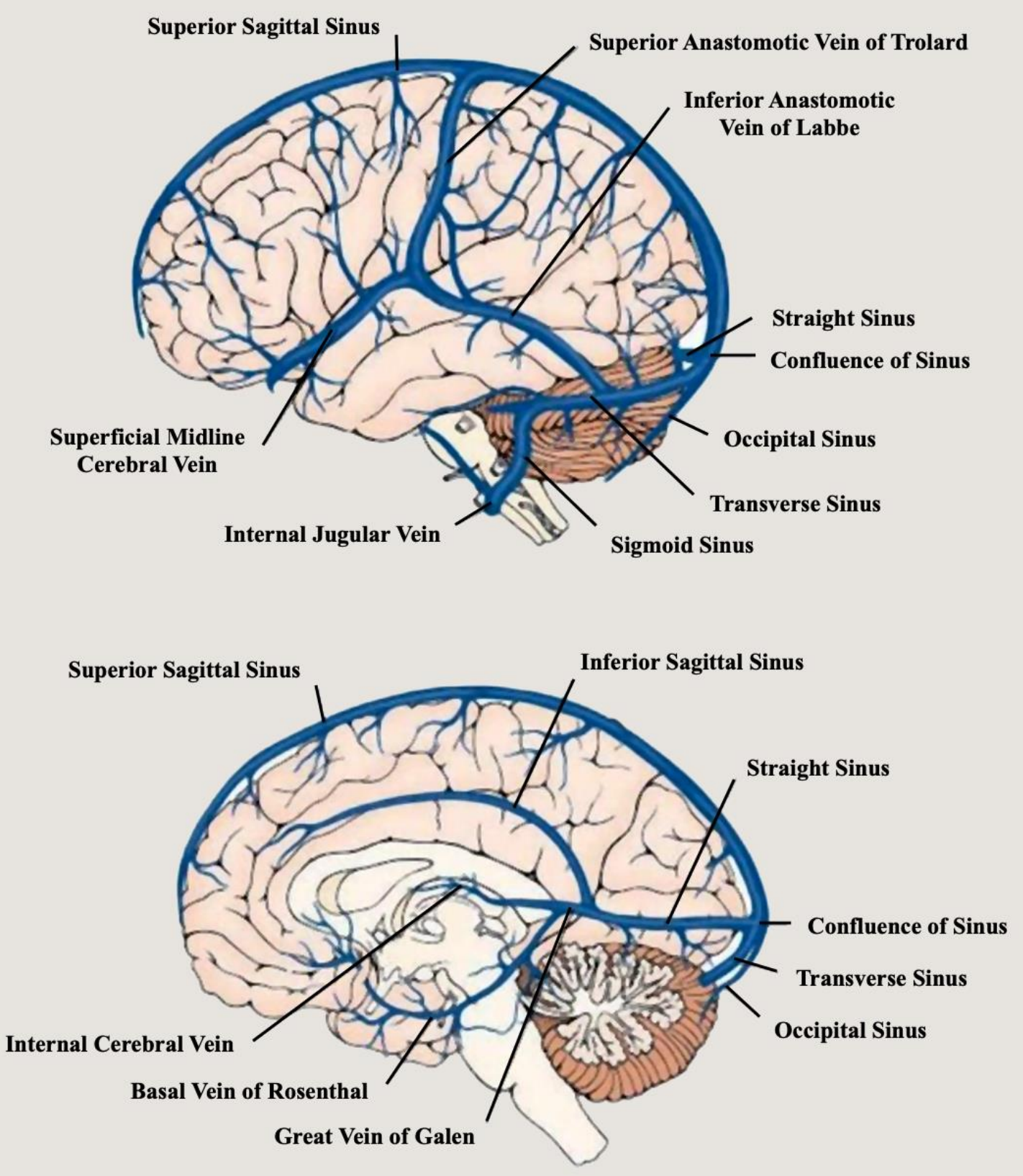

Figure 1.2. Diagram of the major cerebral veins and sinus. Sagittal perspective of the surface of the brain and at the midline of the major cerebral sinus and veins including, superior sagittal sinus, confluence of sinus, straight sinus, sigmoid sinus, transverse sinus, superior anastomotic vein of Trolard, inferior anastomotic vein of Labbe, superficial midline cerebral vein, internal jugular vein, great vein of Galen, basal vein of Rosenthal, and internal cerebral vein. 
is generally considered associated with the endothelium and is often identified as the endothelial glycocalyx. Additionally, the composition of the glycocalyx differs between its endothelial exposed surface and the surface exposed to the vascular lumen. The lumenal surface of the glycocalyx exists in a dynamic state with the soluble components in the blood plasma and flow mediated shearing. Blood plasma derived soluble components are incorporated into a network of proteoglycans, glycoproteins, and glycosaminoglycans to form the glycocalyx as a positive function to blood flow. However, as blood flow increases the shearing off rate of the glycocalyx increases proportionally.

\section{Function of the Glycocalyx}

The endothelial glycocalyx is an influential factor of vascular permeability (Vink and Duling 2000; Henry and Duling 1999). The glycocalyx contributes to endothelial barrier formation via steric and electrostatic hindrance (Ueda et al. 2004). Many of the glycosaminoglycan chains are heavily sulfated resulting in the glycocalyx having a net negative charge (Ueda et al. 2004). Experimental neutralization of the glycocalyx charge increases the uptake of albumin in cultured endothelial cells (Ueda et al. 2004) and increased permeability of fluorescent dextrans (van Haaren et al. 2005). The glycocalyx also functions in repulsing red blood cells from the endothelium (Vink and Duling 1996). The repulsion of red blood cells from the lumenal wall of the endothelium composes a red blood cell (RBC) exclusion zone (Vink and Duling 1996). The interaction between red blood cells and the glycocalyx influence local blood viscosity and blood flow resistance (Pries and Secomb 2003; Lipowsky 2005).

Exposure of the glycocalyx to high shear stress has been reported to result in an increase in the amount of hyaluronan in the glycocalyx by approximately two-fold (Gouverneur et al. 
2006) suggesting a mechanism for sensing shear stress by endothelial cells. Shear stress has also been shown to reduce the thickness of the glycocalyx in the mouse carotid artery whereas regions that maintain laminar flow had a relatively thick glycocalyx layer (van den Berg et al. 2006). Shear stress is thought to be signaled through the glycocalyx core proteins that are then transduced into specific cell signaling processes such as nitric oxide production and reorganization of the cytoskeleton (Tarbell and Pahakis 2006).

\section{Proteoglycans}

Proteoglycans are considered the most important molecular component of the glycocalyx, serving as the major structural component of the matrix, and are termed the "backbone" molecule. A number of variations of proteoglycans exist that differ relative to the specific core protein the molecule contains. The particular core protein that is incorporated into a proteoglycan determines the size and number of glycosaminoglycans that can be attached to the complex and determine whether the complex is attached to the endothelial surface. The core proteins syndecans and glypicans result in a firm attachment to the cell membrane via membrane-spanning domain of a glycosylphosphatidylinositol domain in syndecans or glypicans, respectively (Carey 1997; Fransson et al. 2004). The proteoglycan core proteins mimecan, perlecan, versican, decorin, and biglycan are secreted after their assembly and glycosaminoglycan chain modification, resulting in soluble proteoglycans that diffuse into the blood stream or reside in the glycocalyx (Iozzo 1994; Kinsella et al. 2004). Syndecan-1 proteoglycan is often addressed as a heparin sulfate proteoglycan, while containing similar numbers of heparin sulfate and chondroitin sulfate chains (Mulivor and Lipowsky 2004). Syndecans are stringently regulated by endothelial cell activation and by chemokine signaling (Tkachenko et al. 2005). 
There are five different types of glycosaminoglycan chains, keratan sulfate, heparin sulfate, chondroitin sulfate, dermatan sulfate, and hyaluronan (Funderburgh 2000; Laurent and Fraser 1992). Heparin sulfate, dermatan sulfate, and chondroitin sulfate containing proteoglycans are synthesized in the endoplasmic reticulum and Golgi apparatus of endothelial cells. The glycosaminoglycan chains are composed to linear polymers of disaccharides with variable lengths that are modified by sulfation or deacetylation to a variable extent. The composition of the disaccharides includes uronic acid and hexosamine. The specific classification of glycosaminoglycan depends on the incorporation of uronic acid or hexosamine and the specific pattern of sulfation (Esko and Selleck 2002).

In the vasculature, heparin sulfates proteoglycans represent as much as $50-90 \%$ of the total amount of proteoglycans present in the glycocalyx (Pries et al. 2000; Ihrcke et al. 1993). Chondroitin sulfate is the second most abundant glycosaminoglycan found in the endothelial glycocalyx (Rapraeger et al. 1985). Hyaluronan is a polymeric molecule that does not link to any core proteins but can be found attached to its assembly molecule, hyaluronan synthase that is located on the cytosolic region of the cell membrane (Weigel et al. 1997).

\section{The Glycocalyx in Pathology}

The endothelial glycocalyx provides a number of indispensable roles to blood vessels that are protective, including the regulation on vascular permeability, limiting red blood cell interaction with vessel walls, sensing of shear stress, and signal transduction. Ischemia/reperfusion injury has been shown to reduce the thickness of the glycocalyx in rat mesenteric venioles (Mulivor and Lipowsky 2004) likely caused by the shedding of glycosaminoglycan chains from the glycocalyx. 


\section{Endothelium}

Endothelial cells are the principal cells of vessels and form a layer called the endothelium, which is the innermost cell compartment of all hierarchy of vessels (Figure 1.3). The endothelium forms a size-selective semipermeable barrier between the interstitium and the blood plasma. Endothelial barrier formation regulates the passage of plasma proteins, solutes, and fluid across the endothelium via transcytosis and paracellular permeability. The approximate cell thickness varies from $0.1 \mu \mathrm{m}$ in the capillaries and veins to $1 \mu \mathrm{m}$ in the aorta. Endothelial cells are oriented to the direction of blood flow and overlap immediately adjacent cells of the endothelium. The membranes of adjacent cells are separated by an intercellular space of 15 to $20 \mathrm{~nm}$ is size. The lumenal surface of the endothelium is covered by a glycoprotein coat termed the glycocalyx. The endothelial cells are supported by the basal lamina, an acellular extracellular matrix layer present to all vessels. The basal lamina is located at the basolateral surface of the endothelium, where is provides attachment and supports endothelial processes such as migration and proliferation. In vitro studies have demonstrated that endothelial cells cultured on different basal lamina components produces different rates of gap junctions, cell proliferation, membrane proteins, and actin messenger RNA (Kocher and Madri 1989; Grant et al. 1991).

\section{Endothelial Cell Transport and Barrier Formation}

Endothelial cell transport is a crucial process that maintains the viability of cerebral tissue. Endothelial cell transport mechanisms allow the influx of nutrients and signaling molecules from periphery into the brain via both passive and active mechanisms. The passive movement of solutes from the blood to the extralumenal space occurs via the paracellular pathway - the space between endothelial cells. In contrast, the active movement of molecules 
from blood to the extralumenal space occurs via transcytotic pathway that involves ATPdependent cellular machinery. Paracellular transport and transcellular transport are typically considered to be non-interacting independent pathways that can modulate endothelial barrier function. However, recent progress in the field is beginning to reveal that these pathways cooperate in some physiological processes such as the maintenance of tissue fluid homeostasis.

\section{Paracellular Transport}

Paracellular transport is innately coupled to the permeability of the endothelium and in most cases is an effective transport pathway for solutes smaller than $3 \mathrm{~nm}$ in radius (Komarova and Malik 2010). Therefore, large macromolecules must follow the transcellular transport pathways for entrance to the extralumenal compartment. In paracellular transport, the permeability of the endothelium to solutes is governed by the presence of cell-cell junction proteins that function by holding adjacent endothelial cells together on the endothelium. There are two classes of cell-cell junction proteins present on the endothelial cell membrane that restrict permeability, tight junction proteins and adherens junction proteins (Irudayanathan et al. 2017). While tight junctions and adherens junctions primarily function to promote adhesion of opposing cells in the monolayer and to mediate selective permeability, gap junctions assemble to form channels between immediately adjacent endothelial cells that allow the passage of water, ions, and solutes, and participate in paracellular signaling between endothelial cells. Together, these interendothelial junction proteins form a highly size-selective barrier that can be regulated by modulating the density of these proteins.

\section{Transcellular Transport}

Transcellular transport is an energy-dependent process that can occur via a receptordependent or receptor-independent pathway. The transport of molecules across endothelial 
cells requires an extensive network of cellular machinery that serve in functions such as endocytosis, endocytic trafficking, cargo localization, and exocytosis. The initial step of transcytosis occurs at cholesterol rich invaginations termed caveolae, where a complex chain of molecular events trigger the endocytosis of cargo molecule(s) by caveolar fission from the lumenal surface of the membrane (Minshall et al. 2002; Predescu et al. 2001; Tuma and Hubbard 2003). Endocytosis of the cargo molecule(s) result in the formation of an endocytic vesicle that must enter the correct vesicular trafficking pathway for proper delivery to the ablumenal surface of the cell. After endocytosis, the cargo-containing vesicle enters the secretory pathway and localizes to the appropriate docking site on the inner surface of the ablumenal membrane. Once docked, the cargo can be released by exocytosis via vesicular membrane fusion with the plasma membrane (Minshall et al. 2002). Transcytosis of molecules can be regulated at nearly all stages of the transcellular transport pathway by protein complexes and processes that function in receptor-mediated recruitment of cargo to caveolae domains for endocytosis and/ or vesicle sorting and recycling. As such, the selectivity of molecules that are transported transcellularly by these cellular mechanisms allows the endothelial barrier to maintain a stringently regulated permeability to very specific molecules.

\section{Basal Lamina}

The basal lamina borders the ablumenal surface of endothelial cells and forms an essentially continuous boundary between the endothelium and underlying structures of the intima (Figure 1.3). The basal lamina is a major source of support and attachment of the endothelial layer but also influences vascular permeability and the initiation of blood clotting (Kramer et al. 1984). The basal lamina consists of the lamina rara (inner zone) and the lamina densa (dense fibrillar zone) (Makhoul and Dattilo 1998). The lamina rara is composed 
primarily of the glycoprotein laminin, whereas the lamina densa is composed of collagen type IV. The basal lamina provides a lithe substrate for cell attachment, allowing the endothelium to accommodate mechanical displacement caused by cardiac pulsations and with the torsion of vessels (Ts'ao and Glagov 1970).

The basal lamina is a major source of structural fortification of the vascular wall, primarily attributed by the type IV collagen-rich composition of the lamina densa. The covalently stabilized, polygonal framework of the type IV collagen chains afford the mechanical resilience of the dense fibrillary layer (Wang and Shuaib 2007; Schnittler et al. 1993). Collagen is a proline-rich protein of helical structure and is essential for the attachment of endothelial cells to the subcellular matrix (Madri and Pratt 1986; Ingber and Folkman 1989). Endothelial cells are attached to the collagen component of the lamina densa via self-assembled laminin of the lamina rara (Wang and Shuaib 2007; Schnittler et al. 1993). Fibronectin redistributes mechanical force between the extracellular matrix and the integrin receptors on the surface of vascular smooth muscle cells (vSMC) (Laurent et al. 2005). Microvascular endothelial cells preferentially attach and migrate to fibronectin, opposed to laminin and collagen (Makhoul and Dattilo 1998). Entactin binds both laminin and collagen.

In addition, the basal lamina is composed of glycoproteins, adhesion molecules (laminin, fibronectins, entactin, and thrombospondin), proteoglycans (heparin sulfate), and fibrils (type IV and V collagen) (Schnittler et al. 1993). The reticular layer of the endothelium is composed of type I and III collagen, which are deposited by both vSMCs and endothelial cells. While, type IV collagen is produced exclusively by endothelial cells. 


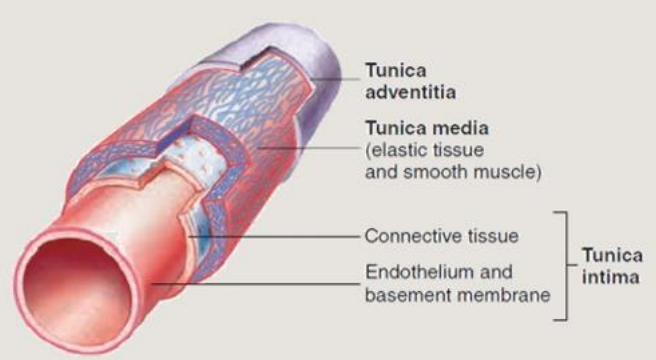

(a) Elastic arteries. The tunica media is mostly elastic connective tissue. Elastic arteries recoil when stretched, which prevents blood pressure from falling rapidly.

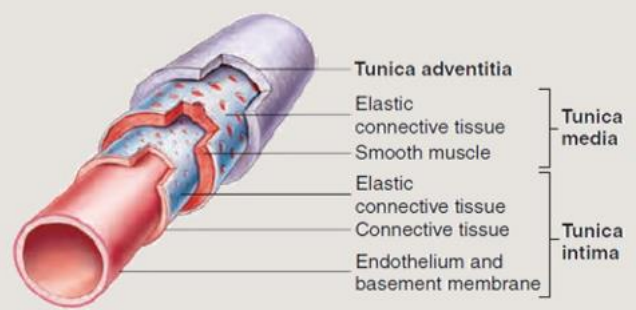

(b) Muscular arteries. The tunica media is a thick layer of smooth muscle. Muscular arteries regulate blood flow to different regions of the body.

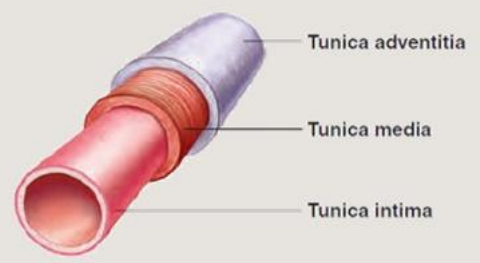

(c) Arterioles. All three tunics are present; the tunica media consists of only one or
two layers of circular smooth muscle cells.

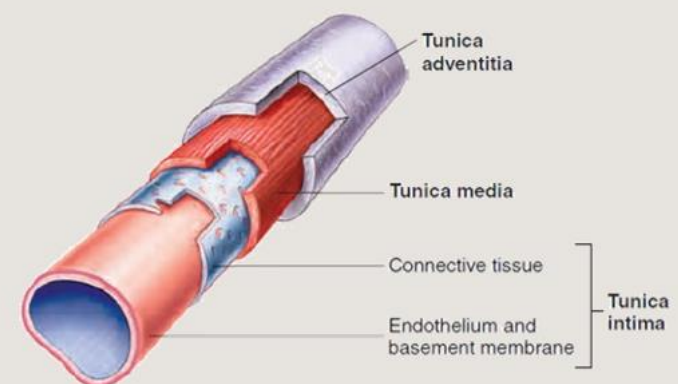

(g) Large veins. All three tunics are present. The tunica media is thin but can regulate vessel diameter because blood pressure in the venous system is low. The predominant layer is the tunica adventitia.

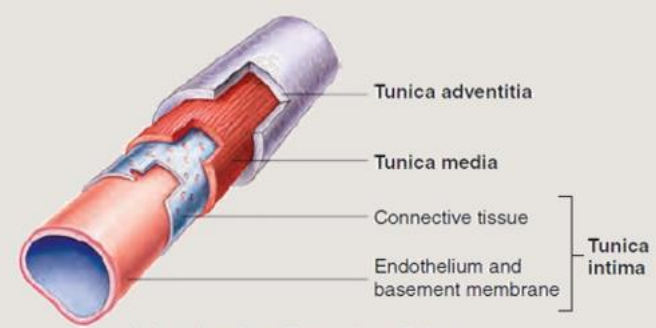

(f) Small and medium veins. All three tunics are present.

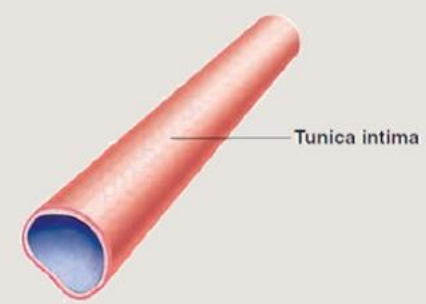

(e) Venules. Only the tunica intima resting on a delicate layer of dense connective tissue is present.

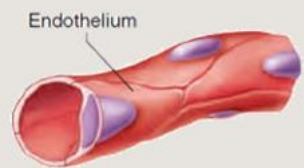

(d) Capillaries. Walls consist of only a simple endothelium surrounded by delicate loose connective tissue.

Figure 1.3. Diagram depicting the layers of the vessel wall specific to vessel hierarchy. The composition of the vessel wall and the specific layers that it comprises provides physical attributes to the vessels related to their function. 


\section{Cellular and Molecular Biology of the Cerebrovascular System}

\section{Endothelial Cell Regulation of the Coagulation Cascade}

Arguably, the most important function mediated by the vascular endothelial cell is the inhibition of the coagulation cascade for the prevention of thrombus formation. The coagulation cascade can be triggered by various forms of physiological stress including; vascular wall injury, circulatory stasis, and hypercoagulable state. For instance, the vasoconstriction caused by a vascular spasm can result in injury to the lining of blood vessels, exposing collagen fibers of the basal lamina to circulating platelets in the lumen of blood vessels. Platelet adherence to collagen fibers forms a 'platelet plug' followed by the deposition of fibrin that forms a mesh (clot).

Initiation of the coagulation cascade is mediated by an enzyme complex consisting of factor VIIa and the tissue factor (TF) catalytic accelerator, which catalyzes the conversion of clotting factor IX and X to coagulation factor IXa and Xa, respectively. Coagulation factor X conversion to activated coagulation factor Xa can occur by the binding of factor IXa to cofactor VIII. Factor $\mathrm{Xa}$ is a prothrombin-converting enzyme to active thrombin, a protease that cleaves fibrinogen, liberating fibrin. Self-assembly of fibrin results in the formation of a mesh, the initial structure of a clot and the core of a thrombus. The fibrin lattice forms on the surface of blood vessels, where it captures circulating erythrocytes and platelets furthering the growth of the thrombus. The fibrin lattice at the core of the thrombus is the therapeutic target of the tissue plasminogen activator (tPA) intervention. 
Interestingly, the head group moiety of the phospholipid, phosphatidylserine is an essential catalytic substrate that is required by the various protease complexes of the coagulation cascade. However, healthy cells stringently maintain phosphatidylserine exclusively to the inner leaflet of the cell membrane. Therefore, the phosphatidylserine must be sourced by a readily available and rapidly modulated mechanism.

Degranulation of platelets provides the phosphatidylserine-rich substrate for the coagulation cascade to occur. For essentially every mechanistic step of the coagulation cascade, vascular endothelial cells provide an inhibitory mechanism that prevents clot formation. Endothelial cells can prevent platelet activation by inhibiting several signaling mechanisms that initiate platelet degranulation: (1) production of ectoenzymes that catabolize the purine nucleotides ATP and ADP; (2) inhibition of thrombin; (3) and prevent the exposure of collagen from the basal lamina. In addition, the release of prostacyclin and nitric oxide (NO) also inhibit platelet activation by antagonizing the activity of the factor VIIa-TF complex, preventing the proteolytic activation of clotting factor IX and $\mathrm{X}$, respectively. Thrombomodulin is an endothelial surface protein that alters the conversion of fibrinogen to fibrin by binding and modifying thrombin, causing it to instead, proteolytically activate protein C. Activated protein C binding to endothelial cell derived protein S inactivates factor VIII and $\mathrm{V}$, inhibiting the actions of coagulation factor IXa and Xa, respectively. Lastly, the liberation of fibrin from fibrinogen can be halted by thrombin inhibition via endothelial cell-derived heparin sulfate-mediated activation of plasma-derived antithrombin III. 


\section{Endothelial Cell Death Mechanisms}

\section{Oxidative Stress}

Reactive oxygen species (ROS) are highly reactive molecules that are generated from the partial reduction of molecular oxygen. Endothelial cells produce several protein catalysts of ROS production; phagocyte oxidase (Phox), NADPD-dependent oxidase (Nox); and in rodents, xanthine oxidase. In endothelial cells, ROS generation may serve as a second messenger signaling molecule that participate in endothelial cell proliferation, barrier formation, vasorelaxation, and vascular remodeling (Rhee et al. 2000). The Nox enzyme has been implicated in the dysfunction of endothelial cell in pathology, including heart failure, cardiac hypertrophy, atherosclerosis, hypertension, and Alzheimer's disease (Keaney 2005).

At low concentrations, $\mathrm{H}_{2} \mathrm{O}_{2}$ can increase the expression of ICAM-1 and the major histocompatibility complex (MHC) class I, but not E-selectin or the vascular cell adhesion molecule-1 (VCAM-1) on the endothelial cell membrane (Bradley et al. 1993). Large amounts of $\mathrm{H}_{2} \mathrm{O}_{2}$ can trigger endothelial cell apoptosis and necrosis. The molecular events leading to endothelial cell apoptosis are mediated by p38 mitogen-activate protein kinase (MAPK) and the c-Jun N-terminal kinase (JNK). Both of which are activated by apoptosis-signaling kinase1 (ASK1)-mediated cascade.

ROS-induced activation of ASK1 can occur via several actions. (1) ROS can lead to a reduced amount of glutathione and thioredoxin in endothelial cells, both of which keep ASK1 inactive upon binding (Song and Lee 2003; Liu and Min 2002). (2) Protein 14-3-3 binds and inhibits ASK1 in endothelial cells that are not activated. Upon activation, ROS can release ASK1 from protein 14-3-3, allowing apoptotic signal transduction (Zhang et al. 1999). (3) Activation of ASK1 can occur by the ROS-mediated activation of protein kinase D (PKD). 
ROS induces the phosphorylation of PKD, triggering its translocation form the surface of the endothelial cell membrane and into the cytosol where is interacts with ASK1 resulting in the autophosphorylation and oligomerization for the activation of ASK1. Experimental inhibition of PDK prevents the ROS-induced activation of ASK1 and JNK, preventing endothelial cell apoptosis (Zhang et al. 2005).

JNK activation via ASK1 is an essential step in the signaling mechanism for endothelial cell apoptosis. The activation of JNK induces the proteolytic activation of Bid, causing its insertion into the mitochondrial membrane where it interacts with the protein Bax. Binding of Bax, causes it dimerization and the initiation of mitochondrial release of several effector molecules. Cytochrome c release from the mitochondria can bind to apoptotic proteaseactivating factor (APAF1), resulting in the activation of procaspase-9. The active caspase-9 results in the activation of effector caspase- 3 and -6 that leads to apoptotic cell death. In addition to cytochrome c, mitochondria release second mediator of apoptotic cell death (SMAD), which augments caspase-9 activation of caspase- 3 by inhibiting the $\mathrm{X}$-linked inhibitor of apoptosis protein, a regulation molecule of caspase-3. Overall, these signaling pathways for endothelial cell apoptosis during oxidative stress indicate a central role for ASK1 in the activation of JNK-Bid/Bax-cytochrome c-dependent apoptosis.

\section{Endoplasmic Reticulum Stress}

The accumulation of misfolded proteins in the endoplasmic reticulum (ER) can trigger a stress response (Marciniak and Ron 2006). Unfolded proteins are sensed by ER resident proteins PKR-like ER kinase (PERK), activating transcription factor 6 (ATF6), and inositolrequiring enzyme 1 (IRE1). These transmembrane proteins contain ER stress sensing domains within the ER lumen and cytosolic domains that transduce signaling that initiate the expression 
of genes involved in protein degradation or apoptotic cell death (Lin et al. 2008). Activation of PERK, a serine/threonine protein kinase, phosphorylates eukaryotic initiation factor $2 \alpha$ (eIF2 $\alpha$ ) that serves as a cofactor to the $80 \mathrm{~S}$ ribosome assembly, thereby preventing ER protein loading by attenuating the synthesis of new proteins. The activation of eIF $2 \alpha$ can also induce the translation of ATF4, which increases the expression of CHOP, a transcription factor that promotes apoptotic cell death. Activated IRE1 cleaves XBP-1 mRNA, producing a transcription factor of genes involved in protein degradation. Activation and binding of IRE1 to TNF receptor-associated factor 2 (TRAF2) couples ER stress to the activation of ASK1JNK (Urano et al. 2000; Matsuzawa et al. 2002).

\section{Metabolic Stress}

Hyperglycemia or the metabolism of free fatty acids can elicit common proinflammatory and oxidative stress signaling. Such as, hyperglycemia can cause the glycation of extracellular plasma proteins and cellular proteins resulting in the formation of advanced glycation end products. Advanced glycation end products bind to the putative receptor on endothelial cells that activate protein kinase $\mathrm{C}$ signaling pathways. In addition, hyperglycemia can upregulate the expression of extracellular matrix and procoagulation proteins, decrease endothelial cell proliferation, inhibit fibrinolysis, and increase endothelial cell apoptosis. Metabolism of sphingolipids results in the production of ceramide that causes endothelial cell death, at low concentrations. The PI3K-Akt pathway is a major pathway in insulin signaling that regulates metabolic function. In endothelial cells, Akt activation causes the phosphorylation of eNOS at Ser-1177, resulting in the release of NO (Fulton et al. 1999; Dimmeler et al. 1999). Mice with dysfunctional Akt-eNOS pathway signaling demonstrate impaired endothelium-dependent vasodilation (Abe et al. 1998). This suggests that metabolic 
stress can have direct consequences on endothelial cell function, including the regulation of blood flow.

\section{The Calcium Channels of Endothelial Cells}

\section{Non-selective Cation Channels}

Non-selective cation channels (NSCC) allow many types of cations through the channel $\left(\mathrm{Na}+, \mathrm{K}+, \mathrm{Ca}^{2+}\right)$. A recent report documents NSCC members of the purinergic ligand-gated receptor channel family in cultured vascular endothelial cells. The P2X4 receptors have been observed in primary cultures of endothelial cells from the microvasculature (Yamamoto et al. 2000b). The P2X4 receptor is functionally involved in ATP and shear stress induced $\mathrm{Ca}^{2+}$ influx (Yamamoto et al. 2000a).

Receptor-activated cation channels (RACC) have been described in endothelial cells. The larger number of these channels are activated by signaling mechanism involving phospholipase C. RACCs that are activated by $\mathrm{Ca}^{2+}$ signaling are also permeable to $\mathrm{Ca}^{2+}$, therefore forming a positive feedback loop for intracellular $\left[\mathrm{Ca}^{2+}\right]$. There is a number of $\mathrm{Ca}^{2+}-$ permeable NSCCs that are activated by vasoactive compounds (Nilius 1990; Nilius et al. 1993a; Nilius and Riemann 1990; Nilius et al. 1993b). The channel-mediated current is slow activating and is only observed under physiological intracellular $\left[\mathrm{Ca}^{2+}\right]$ and is not observed when $\mathrm{Ca}^{2+}$ is buffered (Kamouchi et al. 1999). Additionally, experimentally loading cells with $\mathrm{Ca}^{2+}$ via pipette injection does not cause channel activation and current. NSCC can be activated by the depletion of store operated $\mathrm{Ca}^{2+}$ using store-depletion inhibitor of sarcoplasmic reticulum $\mathrm{Ca}^{2+}$-ATPase (SERCA) pumps with thapsigargin and tert-butyl-benzohydroquinone. The production of inositol 1, 4, 5-triphosphate is critical for NSCC-mediated influx of $\mathrm{Ca}^{2+}$ (Kamouchi et al. 1999). 
Redox-sensitive NSCC respond to oxidative stress-produced superoxide anions, which in turn, activates 28-pS NSCC in cultured endothelial cells. The activated channel produces a current that is equally permeable to $\mathrm{Na}+, \mathrm{K}+$, and $\mathrm{Ca} 2+$. In a study aimed to characterize the mechanism of redox-sensitive NSCC activation in cultured arterial endothelial cells, demonstrated that the administration of the cytosolic oxidant metabolite, oxidized glutathione (GSSG) resulted in channel activation and membrane depolarization, suggesting that membrane depolarization of endothelial cells in vivo occurs during oxidative stress (Koliwad et al. 1996a). In vascular endothelial cell that were exposed to oxidative stress resulted in an increase in intracellular $\mathrm{Na}+$ content and abolished an agonist-stimulated influx of external $\mathrm{Ca}^{2+}$ (Koliwad et al. 1996b). However, in quiescent vascular endothelial cells, oxidative stress induced an increase uptake of $\mathrm{Ca}^{2+}$ when no agonist was administered (Koliwad et al. 1996b). The study noted that the discrepancy of $\mathrm{Ca}^{2+}$ influx may be a direct cause of the membrane depolarization that occurs during oxidant-induced activation of the channel, which may limit $\mathrm{Ca}^{2+}$ influx. Furthermore, the activation and opening of the channel occurs in two states, independent of intracellular $\left[\mathrm{Ca}^{2+}\right]$ and stores (Koliwad et al. 1996b).

\section{Hemodynamics and Hemorheology of the Vascular Hierarchy}

\section{Hemodynamic Force Transduction and Cytosolic Calcium Transients}

Hemodynamic shear stress induces an increase in intracellular $\left[\mathrm{Ca}^{2+}\right]$, which serves as a key regulator of many flow-associated biochemical and physiological processes of the vascular endothelium (Kwan et al. 2003). Furthermore, endothelial cells respond differently to 
varying flow conditions at the level of single-cell oscillations of intracellular $\left[\mathrm{Ca}^{2+}\right]$, which may indicate an endothelial-dependent modulation of cerebrovascular physiology (Helmlinger et al. 1996). The rise in intracellular $\left[\mathrm{Ca}^{2+}\right]$ induced by flow-associated shear stress can potentially originate from the influx of $\mathrm{Ca}^{2+}$ (Yao et al. 2000; Parekh and Penner 1997) or from intracellular $\mathrm{Ca}^{2+}$ stores through activated $\mathrm{IP}_{3}$ or arachidonic acid signaling (Nilius and Droogmans 2001; Parekh and Penner 1997; Prasad et al. 1993; Oike et al. 1994). In a study using rat endothelial cells, chelation of extracellular $\mathrm{Ca}^{2+}$ with EGTA completely attenuated the flow-induced rise of intracellular $\left[\mathrm{Ca}^{2+}\right]$, suggesting that $\mathrm{Ca}^{2+}$ influx alone is responsible for the hemodynamic shear stress-induced $\mathrm{Ca}^{2+}$ transient (Kwan et al. 2003). This observation was consistent with other studies measuring the same phenomenon (Kanai et al. 1995; Schwarz et al. 1992a; Jow and Numann 1999). When intracellular $\mathrm{Ca}^{2+}$ stores are experimentally depleted with inhibitors of endoplasmic reticulum $\mathrm{Ca}^{2+}$-ATPase, flow-induced $\mathrm{Ca}^{2+}$ influx became sensitized (Kwan et al. 2003). This sensitization resulted in an exaggerated increase of $\mathrm{Ca}^{2+}$ influx in response to a relatively modest exposure to shear stress (Kwan et al. 2003). Whereas, the experimental increase of intracellular $\mathrm{Ca}^{2+}$ stores resulted in a reduced flowinduced $\mathrm{Ca}^{2+}$ influx (Kwan et al. 2003).

\section{Hemodynamic Forces Regulate Angiogenesis and Vascular Maintenance Signaling}

Cerebrovascular endothelial cells are continually exposed to shear stress imposed by blood flow, compressive forces exerted by blood pressure, and to tension transmitted through the extracellular matrix (Freund et al. 2012). Laminar flow occurs when movement of a fluid is unidirectional whereas non-laminar flow occurs when the flow of fluid becomes disturbed or turbulent. Physiological levels of shear stress that occur during laminar flow are generally 
thought to be protective of the vasculature (Dimmeler et al. 1996; Yoshizumi et al. 2003). Under physiological conditions, shear stress falls within the range of 1-7 $\mathrm{Pa}$, whereas in veins shear stress ranges $0.1-0.6 \mathrm{~Pa}$ (Malek et al. 1999). Under physiological conditions, shear stress maintains the endothelial lining of blood vessels in a mitotically quiescent state.

Mechanosensory signal transduction of shear stress is thought to be mediated by ion channels (Ando et al. 1988; Nilius and Droogmans 2001; Nilius et al. 1997), cation channels (Schwarz et al. 1992b), and stretch sensitive channels (Nilius et al. 1997). Recent evidence has demonstrated that perturbation of shear stress result in a transient wave of intracellular calcium (Ando et al. 1988) that occur alongside a considerable increase of nitric oxide (NO) production. A study has documented that cultured human umbilical vascular endothelial cells (HUVEC) cell migration along a VEGF gradient is reduced under physiological shear stress equal to what is experienced in the venous system (Song and Munn 2011). Initial gradients of VEGF induced vessel sprouting whereas negative gradients inhibited sprouting but instead assumed a sheetlike growth pattern. This suggests that vascular endothelial cells integrate signals from VEGF gradients and signaling from hemodynamic forces to obtain proper growth and caliber (Song and Munn 2011).The study demonstrated that cells treated with an NOS inhibitor result endothelial cell migration that are insensitive to shear stress. This suggests that NO production under a physiological range serves to maintain vascular endothelial cell in a non-angiogenic state. However, when shear stress exceeds physiological range, endothelial cells respond by entering a state of activated angiogenesis that is mediated by nitric oxide synthase modulation (Zhou et al. 1998; Williams et al. 2006). Experimental inhibition of NOS in vivo has been demonstrated to increase vascular density (Pipili-Synetos et al. 1993). 


\section{Flow-dependent Transcriptional Regulation}

Endothelial cell transcriptome can dramatically change in response to shear stress (Chiu et al. 2005; Ohura et al. 2003; Sun et al. 1993). Cultured HUVEC exposed to different levels of shear stress have shown to alter the regulation of up to 350 genes. Of these, 190 genes were upregulated under laminar flow and 166 genes were found to be down regulated compared to cells maintained under static conditions (Wragg et al. 2014). Of these genes, a pattern of proangiogenic gene expression emerged that was dependent on cell exposure to laminar or stasis flow shear stress. This pattern of gene expression seemed to contrast each other via analogous signaling molecules. Such as, under laminar flow shear stress, VEGF-A is upregulated while shear stress during flow stasis, VEGF-B was upregulated (Carmeliet et al. 1996; Ferrara et al. 1996).

Cultured HUVEC exposed to 1.2 $\mathrm{Pa}$ or $0 \mathrm{~Pa}$ for a period of 24-hours demonstrated as many as 35 microRNA (miRNA) species to be upregulated and as many as 26 miRNAs to be downregulated (Sun et al. 1993), albeit a shear stress force of 1.2 is above physiological range. This shear stress-dependent change in miRNAs is likely contributing to the shift in the transcription profile in these cells after the exposure to shear stress forces.

\section{Flow-dependent Transcription in Pathology}

Several genes regulated by shear stress have been directly linked with vascular pathology. C-type lectin sub family 14 member CLEC14A is expressed 10-fold higher in HUVEC cultured in static conditions compared to cells exposed to shear stress of laminar flow of 2.0 Pa for 24-hours, suggesting it to be a shear stress regulated gene (Mura et al. 2012). CLEC14A is a highly angiogenic molecule that also participates in endothelial cell migration 
and the tube formation of a vessel (Mura et al. 2012). The pathological upregulation of CLEC14A expression has been linked to the development of atherosclerotic plaques and to tumors in the ovaries, bladder, liver, and breast (Khan et al. 2019).

ROBO4 is a vascular specific analogue of the roundabout axon guidance receptor family and has been implicated in tumor development. The function of ROBO4 at the cerebrovasculature is dependent on the activation state of the endothelium (Huminiecki and Bicknell 2000). In cultured HUVEC maintained under static conditions contained a 50-fold greater expression of $\mathrm{ROBO} 4$ compared to cells maintained under laminar shear stress at $2 \mathrm{~Pa}$ for 24-hours (Mura et al. 2012; Sheldon et al. 2009). In activated endothelium, ROBO4 is proangiogenic, participating in endothelial cell migration and vascular tube formation by its expression at the angiogenic sprout in tip cells where it contributes to the formation of filopodia (Sheldon et al. 2009). In contrast, a number of studies have accumulated evidence suggesting that ROBO4 promotes the quiescent state of the endothelium by inhibiting VEGF signaling via ROBO4 interactions with the vascular netrin receptor UNC5B (Marlow et al. 2010). In one study, the inhibition of ROBO4 and UNC5B with antibodies resulted in elevated angiogenesis and structural integrity defects (Marlow et al. 2010). These observations were supported further in a study that documented an elevated level of angiogenesis and degraded vascular barrier integrity in ROBO4 -/- knockout mice (Koch and Haustein 1983). It appears that when endothelial cell are maintained under a shear stress-dependent activated state the function of ROBO4 is antiangiogenic but when endothelial cells are maintained under a static state of low shear stress, ROBO4 functions in a proangiogenic fashion.

TIE1 is an angioprotein receptor that is exclusively expressed on the surface of endothelial cells (Partanen et al. 1992). The pro-inflammatory role of TIE1 is mediated by 
signaling the upregulation of cell adhesion molecules VCAM-1, E-selectin, and ICAM-1 to the lumenal surface of vascular endothelial cells (Chan et al. 2008). TIE1 has been implicated in inflammatory diseases of the endothelium, such as atherosclerosis (Chan et al. 2008; Woo and Baldwin 2011) but also in arthritis and in the pathogenic angiogenesis that occurs in tumors (Kaipainen et al. 1994; Lin et al. 1999; Shahrara et al. 2002). Observations made both in vitro and in vivo suggest TIE1 to be negatively regulated by shear stress caused by laminar flow (Woo and Baldwin 2011; Porat et al. 2004). During angiogenesis, TIE1 is thought to activate endothelial cells causing these cells to exit their quiescent state. Endothelial quiescence and integrity is partially maintained by TIE2 signaling (Papapetropoulos et al. 2000). During angiogenesis, TIE1 dimerizes with TIE2 resulting in the functional inhibition of TIE2 mediated signaling, including the maintenance of endothelial cell quiescence (Seegar et al. 2010).

\section{Cerebrovasculature Malformations}

\section{Hemodynamic Forces of Blood Flow}

The flow of blood through the cerebrovasculature exerts mechanical forces on the wall(s) of blood vessels. The characteristic mechanical force is dependent of the direction in that the redistribution of force occurs on the surface of the vessel wall. A stress is defined as the force per unit area, acting on a surface. The cerebrovasculature is subjected to several forms of stress including, (Aaron and Gosline 1981) longitudinal stress, (Aelvoet et al. 1992) radial stress, and (Albelda and Buck 1990) circumferential stress that result directly by the transport

of blood through the vessel (Patrick and McIntire 1996; Nerem and Girard 1990). The composition of the vascular wall allows blood vessels to accommodate for these mechanical 
disturbances within large vessels. Of larger vessels, the circumferential arrangement of elastic fibers in the tunica media provides structural stability and ductility to the vascular wall (Aaron and Gosline 1981; Dobrin et al. 1990). However, small vessels, such as capillaries do not contain the medial or adventitial layers of the vessel wall. In capillaries including microvessels, structural resilience of the vessel wall is provided by the basal lamina of the intimal layer, containing high levels of type IV collagen (Davies et al. 1994; Dobrin et al. 1990). In addition, endothelial cells of all vessels can mitigate mechanical stress by forming specialized protein complexes that anchor to the cytoskeleton. In vascular endothelial cells, stress fibers are rigid, formed of myosin II and actin filaments that are anchored to the plasma membrane by integrin via linker proteins at regions of tight contact mediated by focal adhesions (Pavalko and Otey 1994). The result of this specific configuration of structural, junctional, and anchoring proteins is the assembly of an intracellular retiform structure that is no longer limited by the structural resilience of the plasma membrane, but by the cytoskeletal protein having the lowest affinity.

In response to acute fluctuations of mechanical stress, such as the stretching of the endothelial cell monolayer, the cytoskeleton and support structures can undergo a realignment to fortify the integrity of the vessel wall and adapt to changes in the direction of mechanical stress (SF et al. 1990; Patrick and McIntire 1996). Blood flow can exert a shear stress on the lumenal surface of vessels and can stimulate endothelial cell elongation, alignment of the cell in the direction of flow, cytoskeletal reorganization, upregulate adhesion molecules, and vessel dilation (Davies et al. 1994; Thurston and Baldwin 1994; Davies and Tripathi 1993; Patrick and McIntire 1996; Nerem and Girard 1990; Resnick and Cimbrone 1995).

In response to chronic states of stress, the size of the vessel lumen and wall thickness can be modulated to the rate of blood flow specific to the vessel (Woollard and Harpman; 
Holman 1965; Hughes 1935). The literature suggests that the modulation of lumen size and wall thickness of vessels occurs to maintain a constant shear stress between $15-20$ dynes $/ \mathrm{cm}^{2}$ for vessel segments and approximately 100 dynes $/ \mathrm{cm}^{2}$ at branch points (Kamiya and Togawa 1980; Nerem and Girard 1990).

\section{Vascular Tortuosity}

The capillary network provides the greatest fluidic resistance to blood flow (Gould et al. 2017; Schmid et al. 2017), reducing the velocity of blood and intralumenal pressure of venous supply. If the hemodynamic forces of blood flow is not dampened and allowed to reach venous circulation, pathogenic manifestations to venous vessels may occur. High flow rates in veins and venioles can injure the vessel wall, causing abnormal deposition of the basement membrane and dysmorphic vascular structure. Degradation of elastin in arterial and arteriolar wall causes aneurysmal dilation and vessel elongation, resulting in vessels becoming tortuous (Dobrin et al. 1988). Elastin deficiency has been implicated in the formation of tortuous vessels in patients with arterial tortuosity syndrome and in transgenic mice (Taarnhøj et al. 2008; Nakamura et al. 2002; Coucke et al. 2006; Yanagisawa et al. 2002; Carta et al. 2009). Interestingly, the degradation of type IV collagen from the basal lamina causes vessels to rupture, but does not cause vessels to become tortuous (Dobrin et al. 1988).

Vessel tortuosity can have deleterious effects to cerebral blood flow. The degree of curvature of a tortuous vessel is proportional to the magnitude of disturbance it imparts to the flow of blood through the affected vessel. The exaggerated curvature of tortuous vessels causes blood flow to become turbulent, increasing the shear stress to local vascular wall. The glycocalyx is a layer of glycoprotein complexes that are formed on the innermost surface of vascular endothelial cells where it comes into direct contact with the flow of blood. A major 
function of the glycocalyx is the formation of an RBC exclusion zone that maintains the laminar flow of blood by repulsing RBC from the surface of endothelial cells. The shear stress resulting from the turbulent flow of blood causes the attrition of the glycocalyx, exposing the surface of endothelial cell membranes. Exposure of the endothelial cell membrane to blood allows recruitment of circulating leukocytes by binding to cell-adhesion molecules on the endothelial cell surface. Binding to cell-adhesion molecules causes leukocyte stalling and inflammation at the site of leukocyte attachment. This localized inflammation initiates several deleterious events; (1) increases vascular permeability; (2) stimulation of the coagulation cascade; (3) and focal damage to tissue.

\section{Capillary-Venous Malformations}

Capillary-venous malformations (CVMs) are collections of enlarged capillaries with irregular structure. The thin walls of CVMs and their apparent lack of elastic component cause leakiness of the malformed network. These capillary networks lack both feeding and draining vessels but instead are contiguous to other malformed capillaries that constitute the network (Rigamonti et al. 1988). The weak structural integrity of these vascular formations results in cerebral hemorrhage in $41 \%$ of persons and focal neurologic deficits in $35-50 \%$ of patients (Brunereau et al. 2000; Siegel 1998; Zabramski et al. 1994; Rigamonti et al. 1987).

\section{Arteriovenous Malformations}

Arteriovenous malformation (AVM) is the pathogenic development of an arteriovenous anastomosis, which is a common configuration of the vascular network, often found in vascular beds of the skin and gut (Hadley 2007). Pathogenic AVMs occur from the improper connection between an artery and a vein, in which blood flow bypasses the capillary 
network; and instead is feed directly into venous supply (Yakes 2004; Frey et al. 2018). Anomalous AVMs form pathways of low fluidic resistance for blood flow between arteries and veins (Yakes 2004). These low resistance pathways permit high velocity blood flow, increasing the shear stress burdened by the vessel wall (Yakes 2004). As fluid tends to flow through the pathway of least resistance, and due to the pressure differential between the feeding artery and vein, a complete lack of capillary perfusion occurs. Low capillary network perfusion can instigate adverse events; including, the degranulation of platelets, initiating the coagulation cascade; the attrition of the glycocalyx, exacerbating blood clot initiation and stimulating local inflammation; and reducing blood flow-dependent gene transcription, causing the destabilization and regression of capillary vessels.

\section{Introduction to Alzheimer's Disease}

Alzheimer's disease is a devastating neurodegenerative disease that affects our most human nature, our cognition. To our current knowledge, $\mathrm{AD}$ is the most complex disease to have ever affected our society. Presently, no effective treatment exists to halt or slow the progression or prevent manifestation of $\mathrm{AD}$.

\section{Amyloid Precursor Protein Processing}

Synthesis of amyloid- $\beta$ occurs via the proteolytic cleavage of the amyloid precursor protein (APP). The gene encoding APP is located on chromosome 21 and contains 18 exons. The APP is a single transmembrane spanning preproprotein that occurs in three major isoforms that are determined by alternative splicing and are identified by the number of amino acids the 
protein contains (695, 751, and 770) (Chasseigneaux and Allinquant 2012). While APP is a ubiquitously expressed protein, there seems to be a cell type specific bias for the particular production of an isoform. Such as, the 695 isoform of APP lacks the encoding regions from exon 7 and 8 , is found highly expressed in neuronal cells and relatively low in non-neuronal cells (Haass et al. 1991; Sandbrink et al. 1994). Whereas, in non-neuronal cell types the predominant variants of APP is the 751 and 770 isoform, which lack exon 8 or the latter contain all 18 exons, respectively (Yoshikai et al. 1990). Noteworthy, the two homologs of APP termed APP like protein 1 and 2 (APLP1 and APLP2) show similar cell type bias, where APLP1 is only found in the brain while APLP2 is ubiquitous (Wasco et al. 1992; Wasco et al. 1993; Coulson et al. 2000). Sequencing data of APP cDNA led to predictions indicating that the protein functions as a 695 amino acid glycosylated integral membrane cell surface receptor protein (Kang et al. 1987). Although, the precise physiological role of this protein is yet to be identified, empirical evidence indicates a possible role in nervous system development, transcription regulation, cell adhesion, synaptic plasticity, axonal transport, and synaptogenesis (Gralle and Ferreira 2007; Chasseigneaux and Allinquant 2012).

The APP protein is classified as a type 1 integral membrane protein characterized by its large extracellular domain, hydrophobic domain, and a relatively short c-terminal intracellular domain (AICD). The large extracellular domain contains several subdomains including, an E1 and E2 region and a Kunitz protease inhibitor (KPI) domain, which is characteristically missing in the 695 isoform of APP (Kang and Müller-Hill 1990; Rohan de Silva et al. 1997). Interestingly, APP isoforms containing the KPI domain are found elevated in AD (Menéndez-González et al. 2005), likely caused by modulated splicing in neurons from isoform 695 of APP to a KPI domain-containing isoform of the protein (Bordji et al. 2010). 
The shift to KPI-containing APP isoforms in neurons is observed following NMDA receptor activation and is temporally associated with elevated $\mathrm{A} \beta$ production by neuronal cells (Bordji et al. 2010).

\section{APP Posttranslational Processing}

APP processing can occur via two alternate pathways involving secretases and proteases, each pathway producing a different final product. In the non-amyloidogenic pathway, $\alpha$-secretase cleaves APP at amino acid number 17 that is located within the A $\beta$ sequence, resulting in the secretion of the soluble APP ( $\operatorname{sAPP} \alpha)$ extracellular domain and an 83 amino acid c-terminal (C83) fragment associated with the membrane. The $\mathrm{C} 83$ fragment is further processed by $\gamma$-secretase to produce the P3 peptide fragment and APP AICD. Processing of APP via the amyloidogenic pathway involves sequential cleavage by $\beta$-secretase and $\gamma$-secretase. Processing by $\beta$-secretase is decisive to whether APP processing occurs through the amyloidogenic or non-amyloidogenic pathway. Cleavage by $\beta$-secretase occurs within the $\mathrm{A} \beta$ sequence at amino acid residue number 1 and 11 of APP resulting in the formation of a soluble APP $\beta$ (SAPP $\beta$ ) product and a membrane-associated 99 amino acid cterminal fragment (C99) (Cole and Vassar 2007). The C99 fragment is further processed by $\gamma$ secretase producing AICD and the pathogenic A $\beta$ peptide (Hartmann et al. 1997).

$\mathbf{S A P Q}$

$\mathrm{sAPP} \alpha$ has been found to participate is a number of physiological functions, including neurite outgrowth, synaptogenesis, and cell adhesion (Mattson 1997; Gakhar-Koppole et al. 2008). In vivo studies with APP deficient mice has shown that administration of sAPP $\alpha$ is effective at ameliorating the abnormalities caused by reduced APP signaling (Ring et al. 2007), indicating that the activity of sAPP $\alpha$ is exerted extracellularly. In addition, studies have found 
that sAPP $\alpha$ functions as a growth factor (HERZOG et al. 2004; Siemes et al. 2006) which regulates adult and embryonic neural stem cell proliferation (Ohsawa et al. 1999; Caille et al. 2004). In vivo, sAPPa is neuroprotective (Mattson et al. 1993; Furukawa et al. 1996; Han et al. 2005; Ma et al. 2009) and can promote learning and memory in animals (Meziane et al. 1998; Taylor et al. 2008).

$s A P P \beta$

Unlike sAPP $\alpha$, the sAPP $\beta$ exerts no neuroprotective effects (Furukawa et al. 1996). Cytotoxicity is also observed from the cleavage product of the $\mathrm{N}$-terminal fragment of sAPP $\beta$, where is serves as a ligand for cell surface death receptor 6 , initiating apoptosis via caspase 6 activation (Nikolaev et al. 2009).

\section{Neurofibrillary Tangles}

Neurofibrillary tangles (NFT) are intracellular basophilic aggregates of filaments in paired helical conformation (PHF) (Kidd 1963). The PHF conformation has a diameter of 10 - $15 \mathrm{~nm}$ and a periodicity of $160 \mathrm{~nm}$ (Kidd 1963). NFTs acquire a morphology that is dependent of the neuron in which it resides. In addition to AD, NFT are found in the CNS of patients affected with subacute sclerosing panencephalitis, postencephalitic Parkinsonism, dementia pugilistica, and the parkinsonian-amyotrophic lateral sclerosis-dementia complex of Guam (Wisniewski et al. 1979). In AD, NFT are composed primarily of the microtubuleassociated protein (MAP) tau (Grundke-Iqbal et al. 1986; Kosik et al. 1986). The hyperphosphorylation of the tau protein causes its polymerization into a filamentous structure. The density of tau inclusions correlates with cognitive decline in AD. 
Microtubule-associated protein(s) are proteins that interact with the microtubules of the cytoskeleton. The MAP tau protein is found abundant in the axons of neurons where it participates in the regulation of axonal transport and stabilizes the tubulin protofilament polymer (Barbier et al. 2019; Binder et al. 1985; Black et al. 1996). Tauopathies are a group of related diseases caused by the loss of function of the tau protein. In addition to AD, tauopathies have been implicated in a number of neurodegenerative diseases including, frontal temporal dementia and progressive supranuclear palsy (Brion et al. 1986). The native conformation of the tau protein is an essentially unfolded state that resembles a random coil configuration (Binder et al. 2005). For tau to undergo polymerization, the protein must undergo major conformational changes. One such conformational states that is able to polymerize is the Alz50 conformation.

In $\mathrm{AD}$-vulnerable neurons, aggregated tau is initially found in the Alz50 state, in which the conformation of the peptide sequence places the amino-terminus in contact with the third microtubule-binding domain (aa 312 - 322). A second conformational change occurs following NFT assembly, in which the Alz50 state undergoes a conformational change to the tau- 66 fold state. The tau- 66 conformation is attained when the proline-rich region of the tau peptide sequence makes contact with the microtubule-binding repeats (MTBR) and partially overlaps the N-terminal binding site (Ghoshal et al. 2001; García-Sierra et al. 2003). Studies suggest that the phosphorylation of tau serves as an inducer for the conformational shift in favor of Alz50 state (García-Sierra et al. 2003). Whereas, C- and/or N-terminal truncation of the peptide favors tau attaining the tau-66 fold state (Binder et al. 2005). 


\section{Vascular Risk Factors}

Past research has firmly documented that vascular related factors increase the risk of developing $\mathrm{AD}$ and other dementias (Mazza et al. 2011). During the previous years, a number of large-scale and long-term population-based clinicopathologic studies have delineated the significant contribution of vascular related risk factors in AD (Kivipelto et al. 2001; Hofman et al. 1997; Skoog et al. 1996; Chui et al. 2006). These studies include Goteborg Study of 70 Year Olds; The Rotterdam Study (Hofman et al. 1997); Honolulu-Asia Aging Study (Launer et al. 2000); The National Heart, Lung, and Blood Institute Twin Study; Studies in Uppsala, Sweden, and Kuopio; Zutphen Elderly Study; Halian Longitudinal Study on Aging; The Framingham Study; The Washington Heights Columbia Aging Study; Nun Study; Chicago Health and Aging Project, and the Bronx Aging Study. Together, these population studies have highlighted the contribution of vascular risk factors to the development of $\mathrm{AD}$ and other dementias, including smoking, diabetes mellitus, hypertension, lipids, hyperinsulinemia, homocysteine, physical inactivity, and fat intake in the development of AD (Santos et al. 2017). The Rotterdam Study was one of the first large-scale studies to investigate the relationship between atherosclerosis and the apolipoprotein E $\varepsilon 4$ allele (ApoE4) with the etiology of $\mathrm{AD}$ and other dementias. The Rotterdam Study revealed an interaction of atherosclerosis with ApoE4 and an association between atherosclerosis and the two major subtypes of dementia, $\mathrm{AD}$ and $\mathrm{VaD}$, but not other forms of dementia (Hofman et al. 1997). The Honolulu-Asia Aging Study (Launer et al. 2000) demonstrated that the risk of developing late-age $\mathrm{AD}$ in patients untreated for hypertension during middle-age increased by $40 \%$, higher than $20 \%$ attributable risk of carrying the $\varepsilon 4$ allele of the APOE gene (Farrer et al. 1997; Slooter et al. 1998). An association between hypertension and the development AD has also 
been reported in a population study in Kuopio and Joensuu, eastern Finland (Kivipelto et al. 2001) and in a 15-year follow up study (Skoog et al. 1996). Postmortem histopathological analysis of patients with $\mathrm{AD}$ and hypertension demonstrate increased deposition of senile plaques and neurofibrillary tangles (Sparks et al. 1995).

The majority of these vascular risk factors have shown to be associated with subcortical lesions, white matter hyperintensities (WMH) (de Leeuw et al. 1999; Liao et al. 1997; Longstreth et al. 1996; Veldink et al. 1998), lacunar infarcts (Bernick et al. 2001; Fisher 1982; Kazui et al. 2000), and cerebral microhemorrhages (Viswanathan and Chabriat 2006). Evidence suggests that therapeutic intervention of hypertension may delay the progression of WMH (Dufouil et al. 2005). Population-based studies have clearly linked cognitive impairment with cognitive decline and the onset of dementia (Longstreth et al. 1996; Burton et al. 2004; de Groot et al. 2000; Swan et al. 1998).

Strong evidence suggests that in the largest group of patients with dementia, $\mathrm{VaD}$ and $\mathrm{AD}$, vascular factors contribute to the disease etiology. Because clinical dementia presents itself as a combination of neurodegenerative and vascular features, the classification criteria used to distinguish $\mathrm{VaD}$ and $\mathrm{AD}$ apart is difficult. Therefore, it is urgent that further research is conducted which aims to identify diagnostic markers in patients with diverse vascular components contributing to the major dementias.

\section{Cerebral Amyloid Angiopathy}

Cerebral amyloid angiopathy (CAA) is a term that describes the pathologic deposition of amyloid- $\beta$ on the walls of cerebral blood vessels. As many as $90 \%$ of patients diagnosed with AD have comorbid CAA and an estimated 30\% of non-demented aged individuals are affected with CAA (Attems 2005; Love et al. 2009; Weller et al. 2009). CAA can occur both 
hereditarily and sporadically and is classified based on the presence or absence of accumulated amyloid- $\beta$ on cerebral capillaries. Different pathological features affect the vasculature depending on the presence or absence of capillary deposition. CAA type 1, common in AD patients, is strongly associated with the apoE4 allele, demonstrates capillary deposition of amyloid- $\beta$, while CAA type 2 does not affect capillaries and only affects larger blood vessels (Attems and Jellinger 2004; Thal et al. 2008).

The precise origin of amyloid- $\beta$ in CAA is unknown. One theory suggests that the source of amyloid- $\beta$ is blood (Glenner et al. 1984). Smooth muscle cells are also thought to be a potential source of amyloid- $\beta$ (Wisniewski and Wegiel 1994). Yet, cerebral capillaries, which lack smooth muscle cells, also present amyloid- $\beta$ accumulation (Herzig et al. 2006). Increasing evidence suggests a neuronal source of amyloid- $\beta$ that accumulates on the vasculature via the perivascular lymphatic fluid where under pathological conditions accumulates (Weller et al. 1998; Burgermeister et al. 2000; Van Dorpe et al. 2000; Herzig et al. 2004; Weller et al. 2008; Vidal et al. 2009).

In human and animal studies have demonstrated pathological alterations of the cerebrovasculature, such as fibrinoid necrosis, microaneurysms, dilation of arteries, and thickening of the tunica media (Maeda et al. 1993; Zekry et al. 2003; Auriel and Greenberg 2012). Blockage of perivascular drainage pathway due to amyloid- $\beta$ may alter the elimination of metabolites from the brain and impair transport of nutrients across the BBB (Weller et al. 2008; Carrano et al. 2012; Hartz et al. 2012). Dyshomeostasis of brain metabolites may lead to cognitive impairment. Vascular damage in proximity to amyloid- $\beta$ deposition is associated with cerebral microbleeds (Verbeek et al. 2009; Schrag et al. 2010). 
In transgenic mice overexpressing human amyloid- $\beta$ in neurons, develop CAA (Calhoun et al. 1999). Experimental evidence suggests that the ratio of $A \beta_{1-40} / A \beta_{1-42}$ is influential to the overall severity of CAA (Herzig et al. 2007; Herzig et al. 2004). In mice constitutively expressing the Dutch type APP mutation, display a high ratio of $A \beta_{1-40} / A \beta_{1-42}$, go on to develop severe CAA (Herzig et al. 2004) while a lower ratio of $\mathrm{A} \beta_{1-40} / \mathrm{A} \beta_{1-42}$ results

in a relatively less severe CAA development (Herzig et al. 2004), indicating that $\mathrm{A} \beta_{1-40}$ and $\mathrm{A} \beta_{1-42}$ can cause CAA onset and that the majority of vascular deposition is from $A \beta_{1-40}$. Although, contrasting data has been reported demonstrating in mice producing solely $A \beta_{1-42}$ develop CAA while mice producing $A \beta_{1-40}$ do not (Kim et al. 2007). These findings may indicate a possible synergistic role of both $A \beta_{1-40}$ and $A \beta_{1-42}$, where the pathogenic interactions of these peptides compromise amyloid- $\beta$ clearance in a self-propagating manner, which causes the development and progression of CAA.

\section{Alignment of Study Aims with Project Goals}

Our motives for conducting this research is to address the shortcomings in the scientific field that focuses on investigating cerebrovascular involvement in the pathogenesis and progression of $\mathrm{AD}$. We have conducted a series of studies that elaborate on the; (1) cellular regulation of mitochondrial dynamics and metabolism after ischemic injury; (2) neuronal injury and degeneration during cerebrovascular hypoperfusion; (3) mechanisms involved in mediating the cytotoxic properties of the $A \beta$ peptide on the vascular endothelium; (4) methodology and analyses necessary for a thorough evaluation of the cerebrovasculature in 
adult mice; and (5) age- and disease-related changes to the cerebral angioarchitecture in the triple transgenic mouse model of AD.

Study 1: Described in chapter 2, we performed a series of experiments to better understand the cellular response to metabolic stress after hypoxia. We focused on the cellular regulation of mitochondrial fission-fusion dynamics and the effects these changes have on mitochondrial function and mitophagy. The aims of this study are in line with the overarching goals of the proposed research project in that the data produced by the study will provide important information regarding the cellular adaptation and deleterious changes to mitochondria during conditions of oxygen deprivation, such as during cerebrovascular hypoperfusion.

Age-related deficiencies in the ability of cells to maintain homeostatic proteostasis can contribute to the sensitivity of cells to protein aggregates present in the extracellular microenvironment (Labbadia and Morimoto 2015). The proteostasis network is facilitated by the lysosomal and endosomal system, where autophagy is a central regulator that maintains homeostatic regulation of this system (Nixon 2013). Neurons with disrupted proteostatic regulation display tau inclusion similar to those in AD (Lambert et al. 2013). Disruptions in autophagy are linked to the pathology of tau. Thus, understanding the mechanistic link between age-related deficiencies in autophagy and the increased burden to the proteostasis network by hypoperfusion-related hypoxia in $\mathrm{AD}$ would provide important insight into the early events in the development of AD. These fundamental observations will provide a critical perspective of the transition of a potentially early reversible event to a chronic, irreversible disease that transcends its dependents on initial factors favoring its development and propagation. 
Astrogliosis is an early event that occurs before the deposition of A $\beta$-plaques in AD (Heneka et al. 2005). However, the relationship between dysfunctional astrocytes and the amyloidosis and tau pathology in AD remain poorly characterized. Astrocytes are heavily involved in $A \beta$ catabolism. In vitro studies have demonstrated that the exposure of astrocytes to $\mathrm{A} \beta$ causes the astrocytes to become activated resulting in the transition of metabolic phenotype, increased inflammatory marker expression, and coordinated calcium signaling (Heneka et al. 2005; Kuchibhotla et al. 2009). Preventing astrocytic activation in APP/PS1 mice accelerates the development of A $\beta$-plaques (Kraft et al. 2013). Damage to the brain can cause reactive astrogliosis (Burda and Sofroniew 2014) that is characterized by abnormal proliferation and hypertrophy. In $\mathrm{AD}$, astrogliosis is associated with downregulated aquaporin 4, which can compromise A $\beta$ clearance via glymphatic flow (Heneka et al. 2005). In the triple transgenic mouse model of $\mathrm{AD}$, astrocytes are atrophied, containing reduced number of branches in the entorhinal cortex at 1-month of age and in the frontal cortex at 3-months of age (Kulijewicz-Nawrot et al. 2012).

The data presented in study 1 provides a description of the changes to astrocytes that can be attributed to hypoperfusion during the early stages of $\mathrm{AD}$, including the induction of autophagy, dysregulated metabolism, and astrocytic activation. These key events are especially important to document, as the pre-symptomatic changes to cellular function that participate in the early pathophysiology of AD remain largely unknown.

Study 2: Chapter 3 describes an in vivo study in mice that aimed to elucidate the neurodegeneration that occurs from the gradual induction of cerebrovascular hypoperfusion. To achieve cerebrovascular hypoperfusion that progresses gradually, we developed a 
procedure that involves the insertion of a microcoil and an ameroid constrictor ring around the common carotid arteries. This surgical procedure resulted in cerebrovascular hypoperfusion that developed over a period of 34-days. We focused on characterizing the damage to the gray and white matter of the brain in hypoperfused mice. The aims of this study were in line with the overarching goals of this research project in that the data provided will inform us of the neurodegeneration in $\mathrm{AD}$ that is attributed to hypoperfusion.

A substantial reduction of cerebral blood flow occurs in humans and in rodents with $\mathrm{AD}$. It is understood that cerebral blood flow begins to decline during the early stages of $\mathrm{AD}$, prior to the deposition of $\mathrm{A} \beta$-plaques and neurofibrillary tangles. The magnitude of hypoperfusion correlates well with the severity of cognitive decline in patients diagnosed with dementia. Although cerebrovascular hypoperfusion is closely associated with cognitive decline in patients, no relationship exists linking region-specific cerebrovascular hypoperfusion with the density of A $\beta$-plaque deposition and NFT formation. This differential association with behavioral phenotypes of $\mathrm{AD}$ and the histopathology of the brain may indicate regional differences of vascular competency and the spare capacity of blood vessels to 'ramp-up' cellular processes during a state of stress. The unique status of age-associated changes to normal cellular efficiency and perseverance of functions performed by blood vessels in an individual may determine if/when a milestone is reached favoring the development of agerelated diseases, such as $\mathrm{AD}$.

Study 3: The study described in chapter 4 aimed to elaborate on the mechanisms of cytotoxicity of $A \beta$ on cerebrovascular endothelial cells. We focused on the metabolic function of endothelial cells following the exposure to $A \beta$ then evaluated mitochondrial function, 
reactive oxygen species production, and calcium dysregulation involved in endothelial cell death. The aims of this study were in line with the research goals of this project in that the data will provide critical information describing the deleterious effects of vascular endothelial cells interaction with $\mathrm{A} \beta$. These observations will also provide an account of the pathogenic changes to vascular endothelial cells following the initial exposure to $A \beta$.

Age-related deficiencies in the ability of the vascular endothelium to maintain physiological function during cell stress can provide the initial sensitivity required to initiate a pathogenic mechanism for the development of age-related diseases. The extent of the agerelated deficiencies of the endothelium may determine the probability of developing AD later in life. This initial insult to the vascular endothelium late in life is likely caused by the $A \beta$ peptide. Endothelial cell injury can result in compromised vascular function, including transendothelial transport. Deficient transendothelial cell transport will lead to a progressive accumulation of $A \beta$ in the parenchyma and on walls of blood vessels, enhancing endothelial cell stress and progressing cellular dysfunction. Obtaining a description of these initial insults to vascular endothelial cells following exposure to $A \beta$ will provide insight into the early insufficiencies of cellular function and reveal reversible events that can lead to the development of novel therapies for the prevention and treatment of clinically diagnosed AD.

Study 4: Described in chapter 5, we performed a study that aimed to develop a procedure that can be used to acquire volumetric data of the cerebrovasculature from the whole-brain. We developed a procedure using a corrosion cast paradigm to create replicas of the cerebrovascular network of the adult mouse brain. Additionally, we optimized an imaging routine that produced a single volumetric dataset of the cerebrovasculature of the entire brain at a resolution 
compatible with the investigation of the microvasculature. We focused on developing the methodology and analyses required to perform a thorough investigation of the cerebrovasculature in adult mice. Our analyses of the angioarchitecture provided a description of the morphometric, topological, and organization of the cerebrovascular network.

Previous methodology applied for the investigation of the cerebrovasculature have largely been limited to relatively small volumetric datasets. This limitation arises from technological constraints that are associated with optical imaging approaches. Common to most imaging paradigms is the tradeoff between resolution and field of view. Therefore, the constraint of acquiring useful imaging data can either be limited in the ability of detecting small structures or limits to the maximum size $(\mathrm{x}, \mathrm{y}, \mathrm{z})$ of the data set. Additionally, the physical properties of tissue can severely restrict the transmission of light into the deep structures of the specimen but can also cause light scattering that reduces the accuracy of image acquisition. Using the corrosion cast method for the cerebrovasculature allowed for the complete removal of tissue around vessels, which considerably enhanced the quality of images collected. These methodological developments described in study 4 are essential for a sufficient assessment of the cerebrovasculature during normal aging and disease.

Study 5: Described in chapter 6 is a study that applied the methodological developments outlined above and covered in detail in chapter 4 to investigate the age- and disease-related changes to the cerebrovasculature. We assessed changes to the angioarchitecture in a triple transgenic mouse model of $\mathrm{AD}$ during age and the progression of the disease. Our analyses focused on characterizing the changes to the microvasculature over the whole-brain and in key brain regions that function in the known cognitive deficits observed in patients with $\mathrm{AD}$. We 
provided a detailed assessment of the fine-scale changes to the microvasculature by measuring the topological and network properties of these vessels. The aims of this study are in line with the overarching goals of this research project by providing information regarding the onset, severity, and pattern of progression of the cerebrovascular degeneration in the triple transgenic mouse model of $\mathrm{AD}$ with age.

Although vascular dysfunction and associated factors are will know to occur at some point during the development of $\mathrm{AD}$, the precise time-course of these changes in $\mathrm{AD}$ remains poorly characterized. These important observations will provide a perspective to how the cerebrovasculature changes during aging and in disease. These details may provide useful biomarkers to identify nascent pathology towards the development of $\mathrm{AD}$ where it can be used to identify abnormal form normal age-related changes to the cerebrovasculature.

\section{References}

Aaron B. B., Gosline J. M. (1981) Elastin as a random-network elastomer: A mechanical and optical analysis of single elastin fibers. Biopolymers 20, 1247-1260.

Abe H., Yamada N., Kamata K., Kuwaki T., Shimada M., Osuga J., Shionoiri F., et al. (1998) Hypertension, hypertriglyceridemia, and impaired endothelium-dependent vascular relaxation in mice lacking insulin receptor substrate-1. J. Clin. Invest. 101, $1784-1788$.

Aelvoet G. E., Jorens P. G., Roelen L. M. (1992) Genetic aspects of the Klippel-Trenaunay syndrome. Br. J. Dermatol. 126, 603-607.

Albelda S. M., Buck C. A. (1990) Integrins and other cell adhesion molecules. FASEB J. 
Off. Publ. Fed. Am. Soc. Exp. Biol. 4, 2868-2880.

Ando J., Komatsuda T., Kamiya A. (1988) Cytoplasmic calcium response to fluid shear stress in cultured vascular endothelial cells. Vitr. Cell. Dev. Biol. J. Tissue Cult. Assoc. 24, 871-877.

Attems J. (2005) Sporadic cerebral amyloid angiopathy: pathology, clinical implications, and possible pathomechanisms. Acta Neuropathol. 110, 345-359.

Attems J., Jellinger K. A. (2004) Only cerebral capillary amyloid angiopathy correlates with Alzheimer pathology--a pilot study. Acta Neuropathol. 107, 83-90.

Auriel E., Greenberg S. M. (2012) The pathophysiology and clinical presentation of cerebral amyloid angiopathy. Curr. Atheroscler. Rep. 14, 343-350.

Barbier P., Zejneli O., Martinho M., Lasorsa A., Belle V., Smet-Nocca C., Tsvetkov P. O., Devred F., Landrieu I. (2019) Role of Tau as a Microtubule-Associated Protein: Structural and Functional Aspects. Front. Aging Neurosci. 11, 204.

Berg B. M. van den, Spaan J. A. E., Rolf T. M., Vink H. (2006) Atherogenic region and diet diminish glycocalyx dimension and increase intima-to-media ratios at murine carotid artery bifurcation. Am. J. Physiol. Heart Circ. Physiol. 290, H915-20.

Bernick C., Kuller L., Dulberg C., Longstreth W. T. J., Manolio T., Beauchamp N., Price T. (2001) Silent MRI infarcts and the risk of future stroke: the cardiovascular health study. Neurology 57, 1222-1229.

Binder L. I., Frankfurter A., Rebhun L. I. (1985) The distribution of tau in the mammalian central nervous system. J. Cell Biol. 101, 1371-1378.

Binder L. I., Guillozet-Bongaarts A. L., Garcia-Sierra F., Berry R. W. (2005) Tau, tangles, and Alzheimer's disease. Biochim. Biophys. Acta 1739, 216-223.

Black M. M., Slaughter T., Moshiach S., Obrocka M., Fischer I. (1996) Tau is enriched on dynamic microtubules in the distal region of growing axons. J. Neurosci. 16, 36013619.

Blinder P., Shih A. Y., Rafie C., Kleinfeld D. (2010) Topological basis for the robust distribution of blood to rodent neocortex. Proc. Natl. Acad. Sci. U. S. A. 107, 12670- 
12675.

Bordji K., Becerril-Ortega J., Nicole O., Buisson A. (2010) Activation of Extrasynaptic, But Not Synaptic, NMDA Receptors Modifies Amyloid Precursor Protein Expression Pattern and Increases Amyloid- Production. J. Neurosci. 30, 15927-15942.

Bradley J. R., Johnson D. R., Pober J. S. (1993) Endothelial activation by hydrogen peroxide. Selective increases of intercellular adhesion molecule-1 and major histocompatibility complex class I. Am. J. Pathol. 142, 1598-1609.

Brion J. P., Flament-Durand J., Dustin P. (1986) Alzheimer's disease and tau proteins. England.

Brundel M., Bresser J. de, Dillen J. J. van, Kappelle L. J., Biessels G. J. (2012a) Cerebral microinfarcts: a systematic review of neuropathological studies. J. Cereb. Blood Flow Metab. 32, 425-436.

Brundel M., Heringa S. M., Bresser J. de, Koek H. L., Zwanenburg J. J. M., Jaap Kappelle L., Luijten P. R., Biessels G. J. (2012b) High prevalence of cerebral microbleeds at 7Tesla MRI in patients with early Alzheimer's disease. J. Alzheimers. Dis. 31, 259-263.

Brunereau L., Labauge P., Tournier-Lasserve E., Laberge S., Levy C., Houtteville J. P. (2000) Familial form of intracranial cavernous angioma: MR imaging findings in 51 families. French Society of Neurosurgery. Radiology 214, 209-216.

Burda J. E., Sofroniew M. V. (2014) Reactive gliosis and the multicellular response to CNS damage and disease. Cell Press.

Burgermeister P., Calhoun M. E., Winkler D. T., Jucker M. (2000) Mechanisms of cerebrovascular amyloid deposition. Lessons from mouse models. Ann. N. Y. Acad. Sci. 903, 307-316.

Burton E. J., Kenny R. A., O’Brien J., Stephens S., Bradbury M., Rowan E., Kalaria R., Firbank M., Wesnes K., Ballard C. (2004) White matter hyperintensities are associated with impairment of memory, attention, and global cognitive performance in older stroke patients. Stroke 35, 1270-1275.

Caille I., Allinquant B., Dupont E., Bouillot C., Langer A., Müller U., Prochiantz A. (2004) 
Soluble form of amyloid precursor protein regulates proliferation of progenitors in the adult subventricular zone. Development 131, 2173-2181.

Calhoun M. E., Burgermeister P., Phinney A. L., Stalder M., Tolnay M., Wiederhold K. H., Abramowski D., et al. (1999) Neuronal overexpression of mutant amyloid precursor protein results in prominent deposition of cerebrovascular amyloid. Proc. Natl. Acad. Sci. U. S. A. 96, 14088-14093.

Carey D. J. (1997) Syndecans: multifunctional cell-surface co-receptors. Biochem. J. 327 ( Pt 1, 1-16.

Carmeliet P., Ferreira V., Breier G., Pollefeyt S., Kieckens L., Gertsenstein M., Fahrig M., et al. (1996) Abnormal blood vessel development and lethality in embryos lacking a single VEGF allele. Nature 380, 435-439.

Carrano A., Hoozemans J. J. M., Vies S. M. van der, Horssen J. van, Vries H. E. de, Rozemuller A. J. M. (2012) Neuroinflammation and blood-brain barrier changes in capillary amyloid angiopathy. Neurodegener. Dis. 10, 329-331.

Carta L., Wagenseil J. E., Knutsen R. H., Mariko B., Faury G., Davis E. C., Starcher B., Mecham R. P., Ramirez F. (2009) Discrete contributions of elastic fiber components to arterial development and mechanical compliance. Arterioscler. Thromb. Vasc. Biol. 29, 2083-2089.

Chan B., Yuan H.-T., Ananth Karumanchi S., Sukhatme V. P. (2008) Receptor tyrosine kinase Tie-1 overexpression in endothelial cells upregulates adhesion molecules. Biochem. Biophys. Res. Commun. 371, 475-479.

Chasseigneaux S., Allinquant B. (2012) Functions of A $\beta$, sAPP $\alpha$ and sAPP $\beta$ : similarities and differences. J. Neurochem. 120, 99-108.

Chi N.-F., Chien L.-N., Ku H.-L., Hu C.-J., Chiou H.-Y. (2013) Alzheimer disease and risk of stroke: a population-based cohort study. Neurology 80, 705-711.

Chiu J.-J., Lee P.-L., Chang S.-F., Chen L.-J., Lee C.-I., Lin K. M., Usami S., Chien S. (2005) Shear stress regulates gene expression in vascular endothelial cells in response to tumor necrosis factor-alpha: a study of the transcription profile with complementary DNA microarray. J. Biomed. Sci. 12, 481-502. 
Chui H. C., Zarow C., Mack W. J., Ellis W. G., Zheng L., Jagust W. J., Mungas D., et al. (2006) Cognitive impact of subcortical vascular and Alzheimer's disease pathology. Ann. Neurol. 60, 677-687.

Cole S. L., Vassar R. (2007) The Alzheimer's disease Beta-secretase enzyme, BACE1. Mol. Neurodegener. 2, 22.

Coucke P. J., Willaert A., Wessels M. W., Callewaert B., Zoppi N., Backer J. De, Fox J. E., et al. (2006) Mutations in the facilitative glucose transporter GLUT10 alter angiogenesis and cause arterial tortuosity syndrome. Nat. Genet. 38, 452-457.

Coulson E. J., Paliga K., Beyreuther K., Masters C. L. (2000) What the evolution of the amyloid protein precursor supergene family tells us about its function. Neurochem. Int. 36, 175-84.

Davies P. F., Robotewskyj A., Griem M. L. (1994) Quantitative studies of endothelial cell adhesion. Directional remodeling of focal adhesion sites in response to flow forces. $J$. Clin. Invest. 93, 2031-2038.

Davies P. F., Tripathi S. C. (1993) Mechanical stress mechanisms and the cell: An endothelial paradigm. Circ Res.

Dimmeler S., Fleming I., Fisslthaler B., Hermann C., Busse R., Zeiher A. M. (1999) Activation of nitric oxide synthase in endothelial cells by Akt-dependent phosphorylation. Nature 399, 601-605.

Dimmeler S., Haendeler J., Rippmann V., Nehls M., Zeiher A. M. (1996) Shear stress inhibits apoptosis of human endothelial cells. FEBS Lett. 399, 71-74.

Dobrin P. B., Schwarcz T. H., Baker W. H. (1988) Mechanisms of arterial and aneurysmal tortuosity. Surgery 104, 568-571.

Dobrin P. B., Schwarcz T. H., Mrkvicka R. (1990) Longitudinal retractive force in pressurized dog and human arteries. J. Surg. Res. 48, 116-120.

Dorpe J. Van, Smeijers L., Dewachter I., Nuyens D., Spittaels K., Haute C. Van Den, Mercken M., et al. (2000) Prominent cerebral amyloid angiopathy in transgenic mice overexpressing the london mutant of human APP in neurons. Am. J. Pathol. 157, 1283- 
1298.

Dufouil C., Chalmers J., Coskun O., Besançon V., Bousser M.-G., Guillon P., MacMahon S., et al. (2005) Effects of blood pressure lowering on cerebral white matter hyperintensities in patients with stroke: the PROGRESS (Perindopril Protection Against Recurrent Stroke Study) Magnetic Resonance Imaging Substudy. Circulation 112, 1644-1650.

Duvernoy H. M., Delon S., Vannson J. L. (1981) Cortical blood vessels of the human brain. Brain Res. Bull. 7, 519-579.

Esko J. D., Selleck S. B. (2002) Order out of chaos: assembly of ligand binding sites in heparan sulfate. Annu. Rev. Biochem. 71, 435-471.

Farrer L. A., Cupples L. A., Haines J. L., Hyman B., Kukull W. A., Mayeux R., Myers R. H., Pericak-Vance M. A., Risch N., Duijn C. M. van (1997) Effects of age, sex, and ethnicity on the association between apolipoprotein $\mathrm{E}$ genotype and Alzheimer disease. A meta-analysis. APOE and Alzheimer Disease Meta Analysis Consortium. JAMA 278, $1349-1356$.

Ferrara N., Carver-Moore K., Chen H., Dowd M., Lu L., O’Shea K. S., Powell-Braxton L., Hillan K. J., Moore M. W. (1996) Heterozygous embryonic lethality induced by targeted inactivation of the VEGF gene. Nature 380, 439-442.

Fisher C. M. (1982) Lacunar strokes and infarcts: a review. Neurology 32, 871-876.

Fransson L.-A., Belting M., Cheng F., Jönsson M., Mani K., Sandgren S. (2004) Novel aspects of glypican glycobiology. Cell. Mol. Life Sci. 61, 1016-1024.

Freund J. B., Goetz J. G., Hill K. L., Vermot J. (2012) Fluid flows and forces in development: functions, features and biophysical principles. Development 139, 12291245.

Frey S., Cantieni T., Vuillemin N., Haine A., Kammer R., Tengg-Kobligk H. von, Obrist D., Baumgartner I. (2018) Angioarchitecture and hemodynamics of microvascular arteriovenous malformations. PLoS One 13, e0203368.

Fulton D., Gratton J. P., McCabe T. J., Fontana J., Fujio Y., Walsh K., Franke T. F., 
Papapetropoulos A., Sessa W. C. (1999) Regulation of endothelium-derived nitric oxide production by the protein kinase Akt. Nature 399, 597-601.

Funderburgh J. L. (2000) Keratan sulfate: structure, biosynthesis, and function. Glycobiology 10, 951-958.

Furukawa K., Barger S. W., Blalock E. M., Mattson M. P. (1996) Activation of K+ channels and suppression of neuronal activity by secreted $\beta$-amyloid-precursor protein. Nature 379, 74-78.

Gakhar-Koppole N., Hundeshagen P., Mandl C., Weyer S. W., Allinquant B., Müller U., Ciccolini F. (2008) Activity requires soluble amyloid precursor protein $\alpha$ to promote neurite outgrowth in neural stem cell-derived neurons via activation of the MAPK pathway. Eur. J. Neurosci. 28, 871-882.

García-Sierra F., Ghoshal N., Quinn B., Berry R. W., Binder L. I. (2003) Conformational changes and truncation of tau protein during tangle evolution in Alzheimer's disease. $J$. Alzheimers. Dis. 5, 65-77.

Ghoshal N., García-Sierra F., Fu Y., Beckett L. A., Mufson E. J., Kuret J., Berry R. W., Binder L. I. (2001) Tau-66: evidence for a novel tau conformation in Alzheimer's disease. J. Neurochem. 77, 1372-1385.

Glenner G. G., Wong C. W., Quaranta V., Eanes E. D. (1984) The amyloid deposits in Alzheimer's disease: their nature and pathogenesis. Appl. Pathol. 2, 357-369.

Gould I. G., Tsai P., Kleinfeld D., Linninger A. (2017) The capillary bed offers the largest hemodynamic resistance to the cortical blood supply. J. Cereb. Blood Flow Metab. 37, $52-68$.

Gouverneur M., Spaan J. A. E., Pannekoek H., Fontijn R. D., Vink H. (2006) Fluid shear stress stimulates incorporation of hyaluronan into endothelial cell glycocalyx. Am. J. Physiol. Heart Circ. Physiol. 290, H458-2.

Gralle M., Ferreira S. T. (2007) Structure and functions of the human amyloid precursor protein: The whole is more than the sum of its parts. Prog. Neurobiol. 82, 11-32. Grant D. S., Lelkes P. I., Fukuda K., Kleinman H. K. (1991) Intracellular mechanisms 
involved in basement membrane induced blood vessel differentiation in vitro. Vitr. Cell. Dev. Biol. J. Tissue Cult. Assoc. 27A, 327-336.

Groot J. C. de, Leeuw F. E. de, Oudkerk M., Gijn J. van, Hofman A., Jolles J., Breteler M. M. (2000) Cerebral white matter lesions and cognitive function: the Rotterdam Scan Study. Ann. Neurol. 47, 145-151.

Grundke-Iqbal I., Iqbal K., Tung Y. C., Quinlan M., Wisniewski H. M., Binder L. I. (1986) Abnormal phosphorylation of the microtubule-associated protein tau (tau) in Alzheimer cytoskeletal pathology. Proc. Natl. Acad. Sci. U. S. A. 83, 4913-4917.

Haaren P. M. A. van, VanBavel E., Vink H., Spaan J. A. E. (2005) Charge modification of the endothelial surface layer modulates the permeability barrier of isolated rat mesenteric small arteries. Am. J. Physiol. Heart Circ. Physiol. 289, H2503-7.

Haass C., Hung A. Y., Selkoe D. J. (1991) Processing of beta-amyloid precursor protein in microglia and astrocytes favors an internal localization over constitutive secretion. $J$. Neurosci. 11, 3783-93.

Hadley G. (2007) Basic Histology. J. Anat. 211, 412-412.

Han P., Dou F., Li F., Zhang X., Zhang Y.-W., Zheng H., Lipton S. A., Xu H., Liao F.-F. (2005) Suppression of Cyclin-Dependent Kinase 5 Activation by Amyloid Precursor Protein: A Novel Excitoprotective Mechanism Involving Modulation of Tau Phosphorylation. J. Neurosci. 25, 11542-11552.

Hartmann T., Bieger S. C., Brühl B., Tienari P. J., Ida N., Allsop D., Roberts G. W., et al. (1997) Distinct sites of intracellular production for Alzheimer's disease A $\beta 40 / 42$ amyloid peptides. Nat. Med. 3, 1016-1020.

Hartz A. M. S., Bauer B., Soldner E. L. B., Wolf A., Boy S., Backhaus R., Mihaljevic I., et al. (2012) Amyloid- $\beta$ contributes to blood-brain barrier leakage in transgenic human amyloid precursor protein mice and in humans with cerebral amyloid angiopathy. Stroke 43, 514-523.

Helmlinger G., Berk B. C., Nerem R. M. (1996) Pulsatile and steady flow-induced calcium oscillations in single cultured endothelial cells. J. Vasc. Res. 33, 360-369. 
Heneka M. T., Sastre M., Dumitrescu-Ozimek L., Dewachter I., Walter J., Klockgether T., Leuven F. Van (2005) Focal glial activation coincides with increased BACE1 activation and precedes amyloid plaque deposition in APP[V717I] transgenic mice. $J$. Neuroinflammation 2, 22.

Henry C. B., Duling B. R. (1999) Permeation of the luminal capillary glycocalyx is determined by hyaluronan. Am. J. Physiol. 277, H508-14.

Herzig M. C., Nostrand W. E. Van, Jucker M. (2006) Mechanism of cerebral beta-amyloid angiopathy: murine and cellular models. Brain Pathol. 16, 40-54.

Herzig M. C., Paganetti P., Staufenbiel M., Jucker M. (2007) BACE1 and mutated presenilin-1 differently modulate Abeta40 and Abeta42 levels and cerebral amyloidosis in APPDutch transgenic mice. Neurodegener. Dis. 4, 127-135.

Herzig M. C., Winkler D. T., Burgermeister P., Pfeifer M., Kohler E., Schmidt S. D., Danner S., et al. (2004) Abeta is targeted to the vasculature in a mouse model of hereditary cerebral hemorrhage with amyloidosis. Nat. Neurosci. 7, 954-960.

HERZOG V., Kirfel G., Siemes C., Schmitz A. (2004) Biological roles of APP in the epidermis. Eur. J. Cell Biol. 83, 613-624.

Hofman A., Ott A., Breteler M. M., Bots M. L., Slooter A. J., Harskamp F. van, Duijn C. N. van, Broeckhoven C. Van, Grobbee D. E. (1997) Atherosclerosis, apolipoprotein E, and prevalence of dementia and Alzheimer's disease in the Rotterdam Study. Lancet (London, England) 349, 151-154.

Holman E. (1965) Abnormal arteriovenous communications. Great variability of effects with particular reference to delayed development of cardiac failure. Circulation 32, 10011009.

Hughes A. F. (1935) Studies on the Area Vasculosa of the Embryo Chick: I. The First Differentiation of the Vitelline Artery. J. Anat. 70, 76-122.5.

Huminiecki L., Bicknell R. (2000) In silico cloning of novel endothelial-specific genes. Genome Res. 10, 1796-1806.

Ihrcke N. S., Wrenshall L. E., Lindman B. J., Platt J. L. (1993) Role of heparan sulfate in 
immune system-blood vessel interactions. Immunol. Today 14, 500-505.

Ingber D. E., Folkman J. (1989) Mechanochemical switching between growth and differentiation during fibroblast growth factor-stimulated angiogenesis in vitro: role of extracellular matrix. J. Cell Biol. 109, 317-330.

Iozzo R. V (1994) Perlecan: a gem of a proteoglycan. Matrix Biol. 14, 203-208.

Irudayanathan F. J., Wang N., Wang X., Nangia S. (2017) Architecture of the paracellular channels formed by claudins of the blood-brain barrier tight junctions. Ann. N. Y. Acad. Sci. 1405, 131-146.

Jellinger K. A. (2010) Prevalence and impact of cerebrovascular lesions in Alzheimer and lewy body diseases. Neurodegener. Dis. 7, 112-115.

Jow F., Numann R. (1999) Fluid flow modulates calcium entry and activates membrane currents in cultured human aortic endothelial cells. J. Membr. Biol. 171, 127-139.

Kaipainen A., Vlaykova T., Hatva E., Böhling T., Jekunen A., Pyrhönen S., Alitalo K. (1994) Enhanced expression of the tie receptor tyrosine kinase mesenger RNA in the vascular endothelium of metastatic melanomas. Cancer Res. 54, 6571-6577.

Kalaria R. N. (2010) Vascular basis for brain degeneration: faltering controls and risk factors for dementia. Nutr. Rev. 68 Suppl 2, S74-87.

Kamiya A., Togawa T. (1980) Adaptive regulation of wall shear stress to flow change in the canine carotid artery. Am. J. Physiol. - Hear. Circ. Physiol. 8, 14-21.

Kamouchi M., Mamin A., Droogmans G., Nilius B. (1999) Nonselective cation channels in endothelial cells derived from human umbilical vein. J. Membr. Biol. 169, 29-38.

Kanai A. J., Strauss H. C., Truskey G. A., Crews A. L., Grunfeld S., Malinski T. (1995) Shear stress induces ATP-independent transient nitric oxide release from vascular endothelial cells, measured directly with a porphyrinic microsensor. Circ. Res. 77, 284 293.

Kang J., Lemaire H.-G., Unterbeck A., Salbaum J. M., Masters C. L., Grzeschik K.-H., Multhaup G., Beyreuther K., Müller-Hill B. (1987) The precursor of Alzheimer's disease amyloid A4 protein resembles a cell-surface receptor. Nature 325, 733-736. 
Kang J., Müller-Hill B. (1990) Differential splicing of Alzheimer's disease amyloid A4 precursor RNA in rat tissues: PreA4(695) mRNA is predominantly produced in rat and human brain. Biochem. Biophys. Res. Commun. 166, 1192-200.

Kazui S., Levi C. R., Jones E. F., Quang L., Calafiore P., Donnan G. A. (2000) Risk factors for lacunar stroke: a case-control transesophageal echocardiographic study. Neurology 54, 1385-1387.

Keaney J. F. J. (2005) Oxidative stress and the vascular wall: NADPH oxidases take center stage. United States.

Khan K. A., McMurray J. L., Mohammed F., Bicknell R. (2019) C-type lectin domain group 14 proteins in vascular biology, cancer and inflammation. FEBS J. 286, 3299-3332.

Kidd M. (1963) Paired helical filaments in electron microscopy of Alzheimer's disease. Nature 197, 192-193.

Kiliç T., Akakin A. (2008) Anatomy of cerebral veins and sinuses. Front. Neurol. Neurosci. 23, 4-15.

Kim J., Onstead L., Randle S., Price R., Smithson L., Zwizinski C., Dickson D. W., Golde T., McGowan E. (2007) Abeta40 inhibits amyloid deposition in vivo. J. Neurosci. 27, $627-33$.

Kinsella M. G., Bressler S. L., Wight T. N. (2004) The regulated synthesis of versican, decorin, and biglycan: extracellular matrix proteoglycans that influence cellular phenotype. Crit. Rev. Eukaryot. Gene Expr. 14, 203-234.

Kivipelto M., Helkala E. L., Laakso M. P., Hänninen T., Hallikainen M., Alhainen K., Soininen H., Tuomilehto J., Nissinen A. (2001) Midlife vascular risk factors and Alzheimer's disease in later life: longitudinal, population based study. BMJ 322, 14471451.

Koch N., Haustein D. (1983) Association of surface IgM with two membrane proteins on murine B lymphocytes detected by chemical crosslinking. Mol. Immunol. 20, 33-37.

Kocher O., Madri J. A. (1989) Modulation of actin mRNAs in cultured vascular cells by matrix components and TGF-beta 1. Vitr. Cell. Dev. Biol. J. Tissue Cult. Assoc. 25, 
$424-434$.

Koliwad S. K., Elliott S. J., Kunze D. L. (1996a) Oxidized glutathione mediates cation channel activation in calf vascular endothelial cells during oxidant stress. J. Physiol. 495 ( Pt 1, 37-49.

Koliwad S. K., Kunze D. L., Elliott S. J. (1996b) Oxidant stress activates a non-selective cation channel responsible for membrane depolarization in calf vascular endothelial cells. J. Physiol. 491 ( Pt 1, 1-12.

Komarova Y., Malik A. B. (2010) Regulation of endothelial permeability via paracellular and transcellular transport pathways. Annu. Rev. Physiol. 72, 463-493.

Kosik K. S., Joachim C. L., Selkoe D. J. (1986) Microtubule-associated protein tau (tau) is a major antigenic component of paired helical filaments in Alzheimer disease. Proc. Natl. Acad. Sci. U. S. A. 83, 4044-4048.

Kraft A. W., Hu X., Yoon H., Yan P., Xiao Q., Wang Y., Gil S. C., et al. (2013) Attenuating astrocyte activation accelerates plaque pathogenesis in APP/PS1 mice. FASEB J. 27, $187-198$.

Kramer R. H., Bensch K. G., Davison P. M., Karasek M. A. (1984) Basal lamina formation by cultured microvascular endothelial cells. J. Cell Biol. 99, 692-698.

Kuchibhotla K. V., Lattarulo C. R., Hyman B. T., Bacskai B. J. (2009) Synchronous hyperactivity and intercellular calcium waves in astrocytes in Alzheimer mice. Science (80-. ). 323, 1211-1215.

Kulijewicz-Nawrot M., Verkhratsky A., Chvátal A., Syková E., Rodríguez J. J. (2012) Astrocytic cytoskeletal atrophy in the medial prefrontal cortex of a triple transgenic mouse model of Alzheimer's disease. J. Anat. 221, 252-262.

Kwan H.-Y., Leung P.-C., Huang Y., Yao X. (2003) Depletion of intracellular Ca2+ stores sensitizes the flow-induced Ca2+ influx in rat endothelial cells. Circ. Res. 92, 286-292.

la Torre J. C. de (2004) Alzheimer's disease is a vasocognopathy: a new term to describe its nature. Neurol. Res. 26, 517-524.

la Torre J. C. de (2010) Vascular risk factor detection and control may prevent Alzheimer's 
disease. Ageing Res. Rev. 9, 218-225.

Labbadia J., Morimoto R. I. (2015) The Biology of Proteostasis in Aging and Disease. Annu. Rev. Biochem. 84, 435-464.

Lambert J. C., Ibrahim-Verbaas C. A., Harold D., Naj A. C., Sims R., Bellenguez C., Jun G., et al. (2013) Meta-analysis of 74,046 individuals identifies 11 new susceptibility loci for Alzheimer's disease. Nat. Genet. 45, 1452-1458.

Launer L. J., Ross G. W., Petrovitch H., Masaki K., Foley D., White L. R., Havlik R. J. (2000) Midlife blood pressure and dementia: the Honolulu-Asia aging study. Neurobiol. Aging 21, 49-55.

Laurent S., Boutouyrie P., Lacolley P. (2005) Structural and genetic bases of arterial stiffness. Hypertens. (Dallas, Tex. 1979) 45, 1050-1055.

Laurent T. C., Fraser J. R. (1992) Hyaluronan. FASEB J. Off. Publ. Fed. Am. Soc. Exp. Biol. 6, 2397-2404.

Leeuw F. E. de, Groot J. C. de, Oudkerk M., Witteman J. C., Hofman A., Gijn J. van, Breteler M. M. (1999) A follow-up study of blood pressure and cerebral white matter lesions. Ann. Neurol. 46, 827-833.

Liao D., Cooper L., Cai J., Toole J., Bryan N., Burke G., Shahar E., Nieto J., Mosley T., Heiss G. (1997) The prevalence and severity of white matter lesions, their relationship with age, ethnicity, gender, and cardiovascular disease risk factors: the ARIC Study. Neuroepidemiology 16, 149-162.

Lin J. H., Walter P., Yen T. S. B. (2008) Endoplasmic reticulum stress in disease pathogenesis. Annu. Rev. Pathol. 3, 399-425.

Lin W. C., Li A. F., Chi C. W., Chung W. W., Huang C. L., Lui W. Y., Kung H. J., Wu C. W. (1999) tie-1 protein tyrosine kinase: a novel independent prognostic marker for gastric cancer. Clin. cancer Res. an Off. J. Am. Assoc. Cancer Res. 5, 1745-1751.

Lipowsky H. H. (2005) Microvascular rheology and hemodynamics. Microcirculation 12, 515.

Liu Y., Min W. (2002) Thioredoxin promotes ASK1 ubiquitination and degradation to inhibit 
ASK1-mediated apoptosis in a redox activity-independent manner. Circ. Res. 90, 12591266.

Longstreth W. T. J., Manolio T. A., Arnold A., Burke G. L., Bryan N., Jungreis C. A., Enright P. L., O’Leary D., Fried L. (1996) Clinical correlates of white matter findings on cranial magnetic resonance imaging of 3301 elderly people. The Cardiovascular Health Study. Stroke 27, 1274-1282.

Love S., Miners S., Palmer J., Chalmers K., Kehoe P. (2009) Insights into the pathogenesis and pathogenicity of cerebral amyloid angiopathy. Front. Biosci. (Landmark Ed. 14, 4778-4792.

Ma T., Zhao Y., Kwak Y.-D., Yang Z., Thompson R., Luo Z., Xu H., Liao F.-F. (2009) Statin's Excitoprotection Is Mediated by sAPP and the Subsequent Attenuation of Calpain-Induced Truncation Events, Likely via Rho-ROCK Signaling. J. Neurosci. 29, $11226-11236$.

Madri J. A., Pratt B. M. (1986) Endothelial cell-matrix interactions: in vitro models of angiogenesis. J. Histochem. Cytochem. Off. J. Histochem. Soc. 34, 85-91.

Maeda H., Matsumoto M., Handa N., Hougaku H., Ogawa S., Itoh T., Tsukamoto Y., Kamada T. (1993) Reactivity of cerebral blood flow to carbon dioxide in various types of ischemic cerebrovascular disease: evaluation by the transcranial Doppler method. Stroke 24, 670-675.

Makhoul R. G., Dattilo J. (1998) The Basic Science of Vascular Disease.

Malek A. M., Alper S. L., Izumo S. (1999) Hemodynamic shear stress and its role in atherosclerosis. JAMA 282, 2035-2042.

Marciniak S. J., Ron D. (2006) Endoplasmic reticulum stress signaling in disease. Physiol. Rev. 86, 1133-1149.

Marlow R., Binnewies M., Sorensen L. K., Monica S. D., Strickland P., Forsberg E. C., Li D. Y., Hinck L. (2010) Vascular Robo4 restricts proangiogenic VEGF signaling in breast. Proc. Natl. Acad. Sci. U. S. A. 107, 10520-10525.

Matsuzawa A., Nishitoh H., Tobiume K., Takeda K., Ichijo H. (2002) Physiological roles of 
ASK1-mediated signal transduction in oxidative stress- and endoplasmic reticulum stress-induced apoptosis: advanced findings from ASK1 knockout mice. Antioxid. Redox Signal. 4, 415-425.

Mattson M. P. (1997) Cellular actions of beta-amyloid precursor protein and its soluble and fibrillogenic derivatives. Physiol. Rev. 77, 1081-1132.

Mattson M. P., Cheng B., Culwell A. R., Esch F. S., Lieberburg I., Rydel R. E. (1993)

Evidence for excitoprotective and intraneuronal calcium-regulating roles for secreted forms of the beta-amyloid precursor protein. Neuron 10, 243-54.

Mazza M., Marano G., Traversi G., Bria P., Mazza S. (2011) Primary cerebral blood flow deficiency and Alzheimer's disease: shadows and lights. J. Alzheimers. Dis. 23, 375389.

Menéndez-González M., Pérez-Pinera P., Martínez-Rivera M., Calatayud M. T., Blázquez Menes B. (2005) APP Processing and the APP-KPI Domain Involvement in the Amyloid Cascade. Neurodegener. Dis. 2, 277-283.

Meziane H., Dodart J.-C., Mathis C., Little S., Clemens J., Paul S. M., Ungerer A. (1998) Memory-enhancing effects of secreted forms of the -amyloid precursor protein in normal and amnestic mice. Proc. Natl. Acad. Sci. 95, 12683-12688.

Minshall R. D., Tiruppathi C., Vogel S. M., Malik A. B. (2002) Vesicle formation and trafficking in endothelial cells and regulation of endothelial barrier function. Histochem. Cell Biol. 117, 105-112.

Mulivor A. W., Lipowsky H. H. (2004) Inflammation- and ischemia-induced shedding of venular glycocalyx. Am. J. Physiol. Heart Circ. Physiol. 286, H1672-80.

Mura M., Swain R. K., Zhuang X., Vorschmitt H., Reynolds G., Durant S., Beesley J. F. J., et al. (2012) Identification and angiogenic role of the novel tumor endothelial marker CLEC14A. Oncogene 31, 293-305.

Nakamura T., Lozano P. R., Ikeda Y., Iwanaga Y., Hinek A., Minamisawa S., Cheng C.-F., et al. (2002) Fibulin-5/DANCE is essential for elastogenesis in vivo. Nature 415, 171175. 
Nerem R. M., Girard P. R. (1990) Hemodynamic influences on vascular endothelial biology. Toxicol. Pathol. 18, 572-582.

Nikolaev A., McLaughlin T., O’Leary D. D. M., Tessier-Lavigne M. (2009) APP binds DR6 to trigger axon pruning and neuron death via distinct caspases. Nature 457, 981-989.

Nilius B. (1990) Permeation properties of a non-selective cation channel in human vascular endothelial cells. Pflugers Arch. 416, 609-611.

Nilius B., Droogmans G. (2001) Ion channels and their functional role in vascular endothelium. Physiol. Rev. 81, 1415-1459.

Nilius B., Droogmans G., Gericke M., Schwarz G. (1993a) Nonselective ion pathways in human endothelial cells. EXS 66, 269-280.

Nilius B., Riemann D. (1990) Ion channels in human endothelial cells. Gen. Physiol. Biophys. 9, 89-111.

Nilius B., Schwartz G., Oike M., Droogmans G. (1993b) Histamine-activated, non-selective cation currents and $\mathrm{Ca} 2+$ transients in endothelial cells from human umbilical vein. Pflugers Arch. 424, 285-293.

Nilius B., Viana F., Droogmans G. (1997) Ion channels in vascular endothelium. Annu. Rev. Physiol. 59, 145-170.

Nixon R. A. (2013) The role of autophagy in neurodegenerative disease. Nature Publishing Group.

Ohsawa I., Takamura C., Morimoto T., Ishiguro M., Kohsaka S. (1999) Amino-terminal region of secreted form of amyloid precursor protein stimulates proliferation of neural stem cells. Eur. J. Neurosci. 11, 1907-1913.

Ohura N., Yamamoto K., Ichioka S., Sokabe T., Nakatsuka H., Baba A., Shibata M., et al. (2003) Global analysis of shear stress-responsive genes in vascular endothelial cells. $J$. Atheroscler. Thromb. 10, 304-313.

Oike M., Droogmans G., Nilius B. (1994) Mechanosensitive Ca2+ transients in endothelial cells from human umbilical vein. Proc. Natl. Acad. Sci. U. S. A. 91, 2940-2944.

Papapetropoulos A., Fulton D., Mahboubi K., Kalb R. G., O’Connor D. S., Li F., Altieri D. 
C., Sessa W. C. (2000) Angiopoietin-1 inhibits endothelial cell apoptosis via the Akt/survivin pathway. J. Biol. Chem. 275, 9102-9105.

Parekh A. B., Penner R. (1997) Store depletion and calcium influx. Physiol. Rev. 77, 901930.

Partanen J., Armstrong E., Mäkelä T. P., Korhonen J., Sandberg M., Renkonen R., Knuutila S., Huebner K., Alitalo K. (1992) A novel endothelial cell surface receptor tyrosine kinase with extracellular epidermal growth factor homology domains. Mol. Cell. Biol. 12, 1698-1707.

Patrick C. W. J., McIntire L. V (1996) Bioengineering contributions in vascular biology at the cellular and molecular level. Trends Cardiovasc. Med. 6, 122-129.

Pavalko F. M., Otey C. A. (1994) Role of Adhesion Molecule Cytoplasmic Domains in Mediating Interactions with the Cytoskeleton. Proc. Soc. Exp. Biol. Med. 205, 282-293.

Pipili-Synetos E., Sakkoula E., Maragoudakis M. E. (1993) Nitric oxide is involved in the regulation of angiogenesis. Br. J. Pharmacol. 108, 855-857.

Porat R. M., Grunewald M., Globerman A., Itin A., Barshtein G., Alhonen L., Alitalo K., Keshet E. (2004) Specific induction of tie1 promoter by disturbed flow in atherosclerosis-prone vascular niches and flow-obstructing pathologies. Circ. Res. 94, $394-401$.

Prasad A. R., Logan S. A., Nerem R. M., Schwartz C. J., Sprague E. A. (1993) Flow-related responses of intracellular inositol phosphate levels in cultured aortic endothelial cells. Circ. Res. 72, 827-836.

Predescu S. A., Predescu D. N., Palade G. E. (2001) Endothelial transcytotic machinery involves supramolecular protein-lipid complexes. Mol. Biol. Cell 12, 1019-1033.

Pries A. R., Secomb T. W. (2003) Rheology of the microcirculation. Clin. Hemorheol. Microcirc. 29, 143-148.

Pries A. R., Secomb T. W., Gaehtgens P. (2000) The endothelial surface layer. Pflugers Arch. 440, 653-666.

Purandare N., Burns A. (2009) Cerebral emboli in the genesis of dementia. J. Neurol. Sci. 
283, 17-20.

Rapraeger A., Jalkanen M., Endo E., Koda J., Bernfield M. (1985) The cell surface proteoglycan from mouse mammary epithelial cells bears chondroitin sulfate and heparan sulfate glycosaminoglycans. J. Biol. Chem. 260, 11046-11052.

Resnick N., Cimbrone M. A. (1995) Hemodynamic forces are complex regulators of endothelial gene expression. FASEB J. 9, 874-882.

Rhee S. G., Bae Y. S., Lee S. R., Kwon J. (2000) Hydrogen peroxide: a key messenger that modulates protein phosphorylation through cysteine oxidation. Sci. STKE 2000, pe1.

Rigamonti D., Drayer B. P., Johnson P. C., Hadley M. N., Zabramski J., Spetzler R. F. (1987) The MRI appearance of cavernous malformations (angiomas). J. Neurosurg. 67, $518-524$.

Rigamonti D., Hadley M. N., Drayer B. P., Johnson P. C., Hoenig-Rigamonti K., Knight J. T., Spetzler R. F. (1988) Cerebral cavernous malformations. Incidence and familial occurrence. N. Engl. J. Med. 319, 343-347.

Ring S., Weyer S. W., Kilian S. B., Waldron E., Pietrzik C. U., Filippov M. A., Herms J., et al. (2007) The Secreted -Amyloid Precursor Protein Ectodomain APPs Is Sufficient to Rescue the Anatomical, Behavioral, and Electrophysiological Abnormalities of APPDeficient Mice. J. Neurosci. 27, 7817-7826.

Rohan de Silva H. A., Jen A., Wickenden C., Jen L. S., Wilkinson S. L., Patel A. J. (1997) Cell-specific expression of beta-amyloid precursor protein isoform mRNAs and proteins in neurons and astrocytes. Brain Res. Mol. Brain Res. 47, 147-56.

Sandbrink R., Masters C. L., Beyreuther K. (1994) Beta A4-amyloid protein precursor mRNA isoforms without exon 15 are ubiquitously expressed in rat tissues including brain, but not in neurons. J. Biol. Chem. 269, 1510-7.

Santos C. Y., Snyder P. J., Wu W. C., Zhang M., Echeverria A., Alber J. (2017) Pathophysiologic relationship between Alzheimer's disease, cerebrovascular disease, and cardiovascular risk: A review and synthesis. Elsevier Inc.

Schaffer C. B., Friedman B., Nishimura N., Schroeder L. F., Tsai P. S., Ebner F. F., Lyden P. 
D., Kleinfeld D. (2006) Two-photon imaging of cortical surface microvessels reveals a robust redistribution in blood flow after vascular occlusion. PLoS Biol. 4, e22.

Schmid F., Tsai P. S., Kleinfeld D., Jenny P., Weber B. (2017) Depth-dependent flow and pressure characteristics in cortical microvascular networks. PLoS Comput. Biol. 13, e1005392.

Schnittler H. J., Franke R. P., Akbay U., Mrowietz C., Drenckhahn D. (1993) Improved in vitro rheological system for studying the effect of fluid shear stress on cultured cells. Am. J. Physiol. 265, C289-98.

Schrag M., McAuley G., Pomakian J., Jiffry A., Tung S., Mueller C., Vinters H. V, et al. (2010) Correlation of hypointensities in susceptibility-weighted images to tissue histology in dementia patients with cerebral amyloid angiopathy: a postmortem MRI study. Acta Neuropathol. 119, 291-302.

Schwarz G., Callewaert G., Droogmans G., Nilius B. (1992a) Shear stress-induced calcium transients in endothelial cells from human umbilical cord veins. J. Physiol. 458, 527538.

Schwarz G., Droogmans G., Nilius B. (1992b) Shear stress induced membrane currents and calcium transients in human vascular endothelial cells. Pflugers Arch. 421, 394-396.

Seegar T. C. M., Eller B., Tzvetkova-Robev D., Kolev M. V, Henderson S. C., Nikolov D. B., Barton W. A. (2010) Tie1-Tie2 interactions mediate functional differences between angiopoietin ligands. Mol. Cell 37, 643-655.

SF G., PS H., JC M., EJ M. (1990) Cyclic Biaxial Strain of Pulmonary Artery Endothelial Cells Causes an Increase in Cell Layer-Associated Fibronectin. Am. J. Respir. Cell Mol. Biol. 3.

Shahrara S., Volin M. V, Connors M. A., Haines G. K., Koch A. E. (2002) Differential expression of the angiogenic Tie receptor family in arthritic and normal synovial tissue. Arthritis Res. 4, 201-208.

Sheldon H., Andre M., Legg J. A., Heal P., Herbert J. M., Sainson R., Sharma A. S., Kitajewski J. K., Heath V. L., Bicknell R. (2009) Active involvement of Robo1 and Robo4 in filopodia formation and endothelial cell motility mediated via WASP and 
other actin nucleation-promoting factors. FASEB J. Off. Publ. Fed. Am. Soc. Exp. Biol. 23, 513-522.

Siegel A. M. (1998) Familial cavernous angioma: an unknown, known disease. Denmark.

Siemes C., Quast T., Kummer C., Wehner S., Kirfel G., Müller U., Herzog V. (2006)

Keratinocytes from APP/APLP2-deficient mice are impaired in proliferation, adhesion and migration in vitro. Exp. Cell Res. 312, 1939-1949.

Skoog I., Lernfelt B., Landahl S., Palmertz B., Andreasson L. A., Nilsson L., Persson G., Odén A., Svanborg A. (1996) 15-year longitudinal study of blood pressure and dementia. Lancet (London, England) 347, 1141-1145.

Slooter A. J., Cruts M., Kalmijn S., Hofman A., Breteler M. M., Broeckhoven C. Van, Duijn C. M. van (1998) Risk estimates of dementia by apolipoprotein E genotypes from a population-based incidence study: the Rotterdam Study. Arch. Neurol. 55, 964-968.

Song J. J., Lee Y. J. (2003) Differential role of glutaredoxin and thioredoxin in metabolic oxidative stress-induced activation of apoptosis signal-regulating kinase 1. Biochem. J. $373,845-853$.

Song J. W., Munn L. L. (2011) Fluid forces control endothelial sprouting. Proc. Natl. Acad. Sci. U. S. A. 108, 15342-15347.

Sparks D. L., Scheff S. W., Liu H., Landers T. M., Coyne C. M., Hunsaker J. C. 3rd (1995) Increased incidence of neurofibrillary tangles (NFT) in non-demented individuals with hypertension. J. Neurol. Sci. 131, 162-169.

Sun Y., Hegamyer G., Colburn N. H. (1993) PCR-direct sequencing of a GC-rich region by inclusion of 10\% DMSO: application to mouse c-jun. Biotechniques 15, 372-374.

Swan G. E., DeCarli C., Miller B. L., Reed T., Wolf P. A., Jack L. M., Carmelli D. (1998) Association of midlife blood pressure to late-life cognitive decline and brain morphology. Neurology 51, 986-993.

Taarnhøj N. C. B. B., Munch I. C., Sander B., Kessel L., Hougaard J. L., Kyvik K., Sørensen T. I. A., Larsen M. (2008) Straight versus tortuous retinal arteries in relation to blood pressure and genetics. Br. J. Ophthalmol. 92, 1055-1060. 
Tarbell J. M., Pahakis M. Y. (2006) Mechanotransduction and the glycocalyx. J. Intern. Med. 259, 339-350.

Taylor C. J., Ireland D. R., Ballagh I., Bourne K., Marechal N. M., Turner P. R., Bilkey D. K., Tate W. P., Abraham W. C. (2008) Endogenous secreted amyloid precursor protein$\alpha$ regulates hippocampal NMDA receptor function, long-term potentiation and spatial memory. Neurobiol. Dis. 31, 250-260.

Thal D. R., Griffin W. S. T., Vos R. A. I. de, Ghebremedhin E. (2008) Cerebral amyloid angiopathy and its relationship to Alzheimer's disease. Acta Neuropathol. 115, 599609.

Thurston G., Baldwin A. L. (1994) Endothelial actin cytoskeleton in rat mesentery microvasculature. Am. J. Physiol. - Hear. Circ. Physiol. 266.

Tkachenko E., Rhodes J. M., Simons M. (2005) Syndecans: new kids on the signaling block. Circ. Res. 96, 488-500.

Tolppanen A.-M., Lavikainen P., Solomon A., Kivipelto M., Soininen H., Hartikainen S. (2013) Incidence of stroke in people with Alzheimer disease: a national register-based approach. Neurology 80, 353-358.

Ts'ao C. H., Glagov S. (1970) Basal endothelial attachment. Tenacity at cytoplasmic dense zones in the rabbit aorta. Lab. Invest. 23, 510-516.

Tuma P. L., Hubbard A. L. (2003) Transcytosis: crossing cellular barriers. Physiol. Rev. 83, 871-932.

Ueda A., Shimomura M., Ikeda M., Yamaguchi R., Tanishita K. (2004) Effect of glycocalyx on shear-dependent albumin uptake in endothelial cells. Am. J. Physiol. Heart Circ. Physiol. 287, H2287-94.

Urano F., Wang X., Bertolotti A., Zhang Y., Chung P., Harding H. P., Ron D. (2000) Coupling of stress in the ER to activation of JNK protein kinases by transmembrane protein kinase IRE1. Science 287, 664-666.

Veldink J. H., Scheltens P., Jonker C., Launer L. J. (1998) Progression of cerebral white matter hyperintensities on MRI is related to diastolic blood pressure. Neurology 51, 
$319-320$.

Verbeek M. M., Kremer B. P. H., Rikkert M. O., Domburg P. H. M. F. Van, Skehan M. E., Greenberg S. M. (2009) Cerebrospinal fluid amyloid $\beta 40$ is decreased in cerebral amyloid angiopathy. Ann. Neurol. 66, 245-249.

Vidal R., Barbeito A. G., Miravalle L., Ghetti B. (2009) Cerebral amyloid angiopathy and parenchymal amyloid deposition in transgenic mice expressing the Danish mutant form of human BRI2. Brain Pathol. 19, 58-68.

Vink H., Duling B. R. (1996) Identification of distinct luminal domains for macromolecules, erythrocytes, and leukocytes within mammalian capillaries. Circ. Res. 79, 581-589.

Vink H., Duling B. R. (2000) Capillary endothelial surface layer selectively reduces plasma solute distribution volume. Am. J. Physiol. Heart Circ. Physiol. 278, H285-9.

Viswanathan A., Chabriat H. (2006) Cerebral microhemorrhage. Stroke 37, 550-555.

Wang C. X., Shuaib A. (2007) Critical role of microvasculature basal lamina in ischemic brain injury. Prog. Neurobiol. 83, 140-148.

Wasco W., Bupp K., Magendantz M., Gusella J. F., Tanzi R. E., Solomon F. (1992) Identification of a mouse brain cDNA that encodes a protein related to the Alzheimer disease-associated amyloid beta protein precursor. Proc. Natl. Acad. Sci. 89, 1075810762.

Wasco W., Gurubhagavatula S., Paradis M. d., Romano D. M., Sisodia S. S., Hyman B. T., Neve R. L., Tanzi R. E. (1993) Isolation and characterization of APLP2 encoding a homologue of the Alzheimer's associated amyloid $\beta$ protein precursor. Nat. Genet. 5, 95-100.

Weigel P. H., Hascall V. C., Tammi M. (1997) Hyaluronan synthases. J. Biol. Chem. 272, 13997-14000.

Weller R. O., Boche D., Nicoll J. A. R. (2009) Microvasculature changes and cerebral amyloid angiopathy in Alzheimer's disease and their potential impact on therapy. Acta Neuropathol. 118, 87-102.

Weller R. O., Massey A., Newman T. A., Hutchings M., Kuo Y. M., Roher A. E. (1998) 
Cerebral amyloid angiopathy: amyloid beta accumulates in putative interstitial fluid drainage pathways in Alzheimer's disease. Am. J. Pathol. 153, 725-733.

Weller R. O., Subash M., Preston S. D., Mazanti I., Carare R. O. (2008) Perivascular drainage of amyloid-beta peptides from the brain and its failure in cerebral amyloid angiopathy and Alzheimer's disease. Brain Pathol. 18, 253-266.

Williams J. L., Weichert A., Zakrzewicz A., Silva-Azevedo L. Da, Pries A. R., Baum O., Egginton S. (2006) Differential gene and protein expression in abluminal sprouting and intraluminal splitting forms of angiogenesis. Clin. Sci. (Lond). 110, 587-595.

Wisniewski H. M., Wegiel J. (1994) Beta-amyloid formation by myocytes of leptomeningeal vessels. Acta Neuropathol. 87, 233-241.

Wisniewski K., Jervis G. A., Moretz R. C., Wisniewski H. M. (1979) Alzheimer neurofibrillary tangles in diseases other than senile and presenile dementia. Ann. Neurol. 5, 288-294.

Woo K. V., Baldwin H. S. (2011) Role of Tie1 in shear stress and atherosclerosis. Trends Cardiovasc. Med. 21, 118-123.

Woollard H. H., Harpman J. A. THE RELATION BETWEEN THE SIZE OF THE ARTERY AND THE CAPILLARY BED IN THE EMBRYO.

Wragg J. W., Durant S., McGettrick H. M., Sample K. M., Egginton S., Bicknell R. (2014) Shear stress regulated gene expression and angiogenesis in vascular endothelium. Microcirculation 21, 290-300.

Yakes W. F. (2004) Endovascular management of high-flow arteriovenous malformations. Semin. Intervent. Radiol. 21, 49-58.

Yamamoto K., Korenaga R., Kamiya A., Ando J. (2000a) Fluid shear stress activates Ca(2+) influx into human endothelial cells via P2X4 purinoceptors. Circ. Res. 87, 385-391.

Yamamoto K., Korenaga R., Kamiya A., Qi Z., Sokabe M., Ando J. (2000b) P2X(4) receptors mediate ATP-induced calcium influx in human vascular endothelial cells. Am. J. Physiol. Heart Circ. Physiol. 279, H285-92.

Yanagisawa H., Davis E. C., Starcher B. C., Ouchi T., Yanagisawa M., Richardson J. A., 
Olson E. N. (2002) Fibulin-5 is an elastin-binding protein essential for elastic fibre development in vivo. Nature 415, 168-171.

Yao X., Kwan H. Y., Chan F. L., Chan N. W., Huang Y. (2000) A protein kinase G-sensitive channel mediates flow-induced $\mathrm{Ca}(2+)$ entry into vascular endothelial cells. FASEB J. Off. Publ. Fed. Am. Soc. Exp. Biol. 14, 932-938.

Yoshikai S., Sasaki H., Doh-ura K., Furuya H., Sakaki Y. (1990) Genomic organization of the human amyloid beta-protein precursor gene. Gene 87, 257-63.

Yoshizumi M., Abe J.-I., Tsuchiya K., Berk B. C., Tamaki T. (2003) Stress and vascular responses: atheroprotective effect of laminar fluid shear stress in endothelial cells: possible role of mitogen-activated protein kinases. J. Pharmacol. Sci. 91, 172-176.

Zabramski J. M., Wascher T. M., Spetzler R. F., Johnson B., Golfinos J., Drayer B. P., Brown B., Rigamonti D., Brown G. (1994) The natural history of familial cavernous malformations: results of an ongoing study. J. Neurosurg. 80, 422-432.

Zekry D., Duyckaerts C., Belmin J., Geoffre C., Moulias R., Hauw J.-J. (2003) Cerebral amyloid angiopathy in the elderly: vessel walls changes and relationship with dementia. Acta Neuropathol. 106, 367-373.

Zhang L., Chen J., Fu H. (1999) Suppression of apoptosis signal-regulating kinase 1-induced cell death by 14-3-3 proteins. Proc. Natl. Acad. Sci. U. S. A. 96, 8511-8515.

Zhang W., Zheng S., Storz P., Min W. (2005) Protein kinase D specifically mediates apoptosis signal-regulating kinase 1-JNK signaling induced by $\mathrm{H} 2 \mathrm{O} 2$ but not tumor necrosis factor. J. Biol. Chem. 280, 19036-19044.

Zhou A., Egginton S., Hudlická O., Brown M. D. (1998) Internal division of capillaries in rat skeletal muscle in response to chronic vasodilator treatment with alpha1-antagonist prazosin. Cell Tissue Res. 293, 293-303. 


\section{CHAPTER 2}

\section{Hypoxia-Reoxygenation of Primary Astrocytes Results in a Redistribution of Mitochondrial Size and Mitophagy}

Dominic D. Quintana, Jorge A. Garcia, Saumyendra N. Sarkar, Sujung Jun, Elizabeth B. Engler-Chiurazzi, Ashley E. Russell, John Z. Cavendish, James W. Simpkins

Center for Basic and Translational Stroke Research

Rockefeller Neuroscience Institute

West Virginia University

Morgantown, West Virginia, 26506

\section{Author Contribution Statement}

DDQ designed studies, conducted studies and composed the manuscript. JAG, AER and JZC conducted studies. SNS, SJ and EBEC revised the manuscript. JWS designed studies and revised the manuscript. 


\section{Abstract}

Astrocytes serve to maintain proper neuronal function and support neuronal viability, but remain largely understudied in research of cerebral ischemia. Astrocytic mitochondria are core participants in the metabolic activity of astrocytes. The objective of this study is to assess astrocyte mitochondrial competence during hypoxia and post-hypoxia reoxygenation and to determine cellular adaptive and pathological changes in the mitochondrial network. We hypothesize that during metabolic distress in astrocytes; mitochondrial networks undergo a shift in fission-fusion dynamics that results in a change in the morphometric state of the entire mitochondrial network. This mitochondrial network shift may be protective during metabolic distress by priming mitochondrial size and facilitating mitophagy. We demonstrated that hypoxia and post-hypoxia reoxygenation of rat primary astrocytes results in a redistribution of mitochondria to smaller sizes evoked by increased mitochondrial fission. Excessive mitochondrial fission corresponded to Drp-1 dephosphorylation at Ser 637, which preceded mitophagy of relatively small mitochondria. Reoxygenation of astrocytes marked the initiation of elevated mitophagic activity primarily reserved to the perinuclear region where a large number of the smallest mitochondria occurred. Although, during hypoxia astrocytic ATP content was severely reduced, after reoxygenation ATP content returned to near normoxic values and these changes mirrored mitochondrial superoxide production. Concomitant with these changes in astrocytic mitochondria, the number of astrocytic extensions declined only after 10-hours post-hypoxic reoxygenation. Overall, we posit a drastic mitochondrial network change that is triggered by a metabolic crisis during hypoxia; these changes are followed by mitochondrial degradation and retraction of astrocytic extensions during reoxygenation. 


\section{Keywords}

Astrocytes, Mitochondria, Oxygen Deprivation, Fission and Fusion, Mitophagy, Drp-1, LC3 


\section{Introduction}

Stroke is a cerebrovascular event characterized by severe cerebral ischemia that is the fifth leading cause of death and is the primary contributor of adult disability in the U.S. (Kochanek et al., 2014; Mozaffarian et al., 2015). During stroke there is a cerebral cellular energy crisis caused by a decline in the delivery of the substrates, glucose and molecular oxygen, resulting in a compromised synthesis of ATP through a collapse of oxidative phosphorylation and glycolysis (Rossi et al., 2007). Insufficient cellular ATP content interrupts a wide range of indispensable ATP-dependent processes, including ion balances across neuronal membranes (Hansen and Nedergaard, 1988; Silver et al., 1997). An elevation of free cytosolic $\mathrm{Ca}^{2+}$ levels via voltage-gated and receptor-gated calcium channels is the central effector initiating the massive release of extracellular glutamate, and the primary cause of excitotoxicity in ischemia (Katayama et al., 1991; Duffy and MacVicar, 1996; Parpura and Haydon, 2000). Clearance of synaptic glutamate is a core function of astrocytes that helps protect against glutamate toxicity (Rothstein et al., 1996).

Also, astrocytes are critical for providing neurons with a source of glutamine necessary for glutamate production through a process known as the glutamate-glutamine cycle (Waniewski and Martin, 1986; Chaudhry et al., 2002). Embargo of glutamine delivery to neurons by the blockade of astrocytic conversion of glutamate to glutamine has been reported to reduce the potassium-evoked glutamate release in experimental models of focal ischemia, reducing infarct size (Paulsen and Fonnum, 1989; Swanson et al., 1990). Astrocytic mitochondria are key organelles that allow astrocytes to participate in such extensive metabolic activities (Nehlig et al., 2004; Lovatt et al., 2007). 
Here we investigated the effects of hypoxia and post-hypoxia reoxygenation on astrocytic mitochondrial structure, including mitochondrial dimensions and content, as well as the underlying mechanism(s) and functions of these dynamic changes. This research provides evidence of early mitochondrial fission during hypoxia-reoxygenation that may participate in the damaging effects of ischemic insult to the central nervous system.

\section{Materials and Methods}

\section{Preparation of Primary Astrocytic Cultures}

Primary astrocytic cultures were prepared using a method previously reported, which generated an $85 \%$ yield of astrocytes, and a $15 \%$, and $<1 \%$ yield of progenitor cells and microglia, respectively (Almeida and Medina, 1998). Embryonic day 19 pregnant rats were deeply anesthetized with isoflurane. After confirming deep anesthetization via tail pinch, rats received a 3-inch vertical incision to the lower abdomen. Once the incision was made, the embryos including the placenta and amniotic sacks were extracted from the uterus. Each embryo was removed from the amniotic sack by creating a small 0.5 -centimeter incision and gently palpating the fetus out. As the fetuses were extracted from the amniotic sack, the heads were removed with sharp scissors and placed in ice cold HBSS buffer (HyClone, Thermo Fisher Scientific, Waltham, MA) while the remaining fetus were prepared. Fetal brains were removed by making a small incision across the sagittal suture. Once the brains were removed, the cerebellum was removed and discarded. The remaining brain tissue was removed from the meninges with small forceps. The meninges free tissue was placed into a 50 milliliter centrifuge tube containing ice cold Dulbecco's modified Eagle's medium (DMEM) (HyClone, 
Thermo Fisher Scientific, Waltham, MA). Once all fetal brains were removed, the tissue was centrifuged at 10,000 times gravity for 3 minutes and subsequently resuspended in ice cold DMEM (HyClone, Thermo Fisher Scientific, Waltham, MA). Tissue was then minced by pipetting to dissociate aggregations of cells. Dissociated cells were then filtered through a 40micron filter then cultured in DMEM (HyClone, Thermo Fisher Scientific, Waltham, MA) supplemented with $10 \%$ fetal bovine serum and $1 \%$ penicillin streptomycin for 7 days to allow sufficient time for cellular differentiation. After the growth phase, primary astrocytes were plated on 6 centimeter culture dishes, 96-well plates, or 6-well culture plates containing sterilized 22 X $22 \mathrm{~mm}$ glass coverslips at a seeding density of 200,000, 10,000, and 50,000 cells per well, respectively. Primary astrocytes were allowed 24-hours of growth before labeling procedure.

\section{Hypoxia Treatment and Imaging Preparation}

Mitochondrial labeling preceded experimental treatment. Mitochondrial labeling in all three experimental conditions was done at the same time so that any time-relevant effects of the labeling procedure would occur uniformly. Mitochondrial labeling was performed with 70nM of MitoTracker Red CMXRos (Invitrogen, Molecular Probes, Eugene, OR) diluted in prewarmed $\left(37^{\circ} \mathrm{C}\right)$ DMEM culture medium then allowed to incubate with primary astrocytes for 20 minutes before washing 2 times with prewarmed DMEM. Hypoxic treatment was administered as $0.4 \%$ oxygen in nitrogen gas balance (Airgas USA, LLC, St. Louis, MO) for 3 hours while incubated at $37{ }^{\circ} \mathrm{C}$. After the 3 hours of hypoxia, primary astrocytes were removed from the hypoxic enclosure and allowed a 10-hour reoxygenation period at normoxic, atmospheric oxygen level. The experimental timeline was designed so that all experimental groups completed treatment at the same time. After treatment, coverslips containing astrocytes 
were washed once with $0.1 \mathrm{M}$ phosphate buffer saline (PBS) at physiological $\mathrm{pH}$ then fixated with $3.7 \%$ paraformaldehyde at physiological $\mathrm{pH}$ for 15 minutes then subsequently washed 3 times with 0.1M PBS for 5 minutes. After washing, free aldehyde groups were quenched with $0.3 \mathrm{M}$ glycine in $0.1 \mathrm{M}$ PBS at $7.4 \mathrm{pH}$ for 10 minutes followed by washing 2 times for 5 minutes. Coverslips containing astrocytes were mounted on glass specimen slides with a medium containing 3\% polyvinyl alcohol/ glycerin/ Tris-HCl / DABCO.

\section{Image Acquisition and Analysis}

Microscopy images were acquired with a Zeiss LSM 510 violet confocal microscope with a $63 \mathrm{X}$ magnification oil objective running on a Zen platform. Several criteria were developed to select astrocytes for inclusion in the study. First, astrocytes were inspected and, astrocytes that did not show signs of imminent death or nascent apoptosis were selected; second, astrocytes had to show canonical morphology, including well-developed extensions and appropriate size; and third, astrocytes had to be adequately spaced apart so that a single astrocyte's boundaries could easily be determined. Seven normoxic, six hypoxic, and six hypoxic-reoxygenation astrocytes were selected from 3 cultures per group for a detailed assessment of mitochondria structure features. Image analysis was conducted with ImageJ software (ImageJ 1.48v, Wayne Rasband, National Institutes of Health, USA) using the particle analysis function to provide mitochondrial estimates. The following parameters were determined: area distributions, length, diameter, area, total mitochondrial mass, and roundness. Mitochondrial size distributions were calculated by sorting the entire content of astrocytic mitochondria into specific area categories $(0.004-0.79,0.80-1.59,1.60-2.39,2.40-3.19,>3.20$ $\mu \mathrm{m}^{2}$ ). Once the total number of mitochondria have been sorted into their respective size categories, the number of mitochondria that fell within each size range was counted; these 
numbers were used to calculate the percentage of total mitochondria that fall within each size range by dividing the sum of mitochondria per size range by the total number of mitochondria in the astrocyte. Mitochondrial mass was calculated by summating the total area of mitochondria for each astrocyte then dividing this by the number of astrocytes for that group. Mitochondrial aspect ratio $(\mathrm{AR}=$ major axis/ minor axis) and form factor $(\mathrm{FF}=1 / 4 \mathrm{x}$ (area / perimeter $\left.{ }^{2}\right)$ ) were used to describe the full array of mitochondrial size and complexity as

previously reported (Koopman et al., 2005b). Average astrocytic area and astrocytic extensions were calculated to determine the effects of hypoxia and post-hypoxic reoxygenation on astrocytic morphology. Astrocytic extensions were classified into three categories, primary, secondary, and tertiary. Extensions with origins of the soma were deemed primary extensions, extensions with origins of primary extensions were termed secondary extensions, and extensions with origins of secondary extensions were termed tertiary extensions.

\section{Three-Dimensional Surface Reconstruction}

Three-dimensional surface reconstructions of deconvolved primary z-stack data sets collected by confocal microscopy were generated by Imaris (Bitplane). Z-stacks were collected on a Zeiss LSM 710 confocal microscope running on the Zen 2 platform with a C-Apochromat $63 \mathrm{x} / 1.20$ objective. Parameters for z-stack image collection were as follows, a pinhole set to 0.7 Airy units, a frame size of 4096 X 4096, and a pixel dwell of $0.79 \mu$ s to generate 16-bit images with a digital zoom of 1 . Images in z-stacks were set to be collected at a step size of $0.100 \mu \mathrm{m}$ for the entire thickness of the cell. 


\section{Western Blots}

Primary astrocytes were cultured in 6 centimeter culture dishes $(n=5 /$ group) in DMEM supplemented with $10 \%$ FBS and $1 \%$ penicillin streptomycin at a seeding density of $\approx 200,000$ cells per dish. After 72-hours, astrocytes were either maintained at normoxia, exposed to 3-hours of hypoxia, or 3-hours hypoxia then 10-hours reoxygenation. To determine autophagic flux, an LC3 turnover assay was performed (Mizushima et al., 2010). Lysosomal degradation of LC3 II was inhibited by treating cells with $30 \mu \mathrm{M}$ Chloroquine (Sigma-Aldrich,

St. Louis, MO) diluted in DMEM prior to hypoxic exposure. Astrocytes treated with $10 \mu \mathrm{M}$ forskolin or $10 \mu \mathrm{M}$ rolipram were used as positive controls for phosphorylated Drp-1 at Ser637. After exposure, cells were lifted from culture dishes in $4 \mathrm{~mL}$ of $0.1 \mathrm{M}$ PBS then collected into $15 \mathrm{~mL}$ conical tubes. Suspended cells were pelleted by centrifugation at $3000 \mathrm{rpm}$ for 2minutes. After centrifugation, supernatant was decanted and discarded. Primary astrocyte pellets were lysed with $250 \mu \mathrm{L}$ RIPA buffer ( $0.5 \%$ deoxycholate/ $0.1 \%$ sodium dodecyl sulfate/ $150 \mathrm{mM}$ sodium chloride/ 50mM ( $\mathrm{pH} 8.0)$ Tris- $\mathrm{HCl}$ ) supplemented with $2 \mu \mathrm{L} / \mathrm{mL}$ protease and phosphatase inhibitors (EMD Millipore, Billerica, MA). Astrocytic lysates were then briefly sonicated then centrifuged at $12,000 \mathrm{rpm}$ for 20-minutes to pellet cellular debris, supernatant was collected in new $2 \mathrm{~mL}$ microcentrifuge tubes. Protein concentrations were estimated using Pierce Protein Assay Reagent (Thermo Scientific, Rockford, IL) to normalize protein content variability between culture dishes. Lysates were prepared in 4X Laemmle buffer containing $10 \% \beta$ mercaptoethanol and placed in a heated water bath for 10 -minutes to denature the protein samples. Protein samples were resolved by SDS-PAGE 4-20\% precast gels, MiniPROTEAN TGX (Bio Rad, USA) then transferred onto a PVDF Immunobilon membrane (EMD Millipore, Billerica, MA). Membranes were blocked with blocking buffer (LI-COR 
Bioscience, Lincoln, NE) supplemented with $0.1 \%$ Tween 20 for 45 -minutes. Membranes were incubated with affinity purified primary antibodies raised in rabbit or mouse targeted against phosphorylated-dynamin related protein 1 at Ser 637 (pDrp-1), Drp-1 (Cell Signaling, Danvers, MA), microtubule-associated 1A/1B-light chain 3 (LC3) (Sigma-Aldrich, St. Louis, MO), and $\beta$-actin (Santa Cruz Biotechnology, Dallas, TX) at a dilution of 1:500 for 24-hours at $4{ }^{\circ} \mathrm{C}$. Membranes were washed 3 times for 15 -minutes with TBST (20mM Tris- $\mathrm{HCl} / 500 \mathrm{mM}$ $\mathrm{NaCl} / 0.1 \%$ Tween 20) solution then incubated with IRDye 800CW and IRDaye 680RD (LICOR Biosciences, Lincoln, NE) secondary goat antibodies targeted against rabbit or mouse IgG (Molecular Probes, Eugene, OR) for 4-hours. Blots were washed 5 times for 15-minutes with TBST then fluorescence imaged with an Odyssey imaging system (LI-COR Biosciences, Lincoln, NE). Blot images were analyzed using Image Studio Lite 4.0 (LI-COR Biosciences, Lincoln, NE) then analyzed for fluorescence intensity normalized to $\beta$-actin.

\section{Mitochondrial Autophagy Measurement}

Primary astrocytes were plated in 6-well culture plates containing $22 \mathrm{~mm} \mathrm{X} \mathrm{22mm} \mathrm{glass}$ cover slips at a seeding density of 50,000 cells per well and incubated at $37{ }^{\circ} \mathrm{C}$ for 24 -hours. Prior to hypoxic treatment, astrocytic mitochondria were labeled with 70nM of MitoTracker Red CMXRos for 20-minutes then washed twice with pre-warmed $\left(37^{\circ} \mathrm{C}\right)$ DMEM culture medium. After receiving hypoxic or hypoxic reoxygenation treatment, cells were rinsed with ice-cold 0.1M PBS at physiological pH then quickly fixated with 3.7\% paraformaldehyde at physiological pH. Post-fixation, cells were washed 3 times for 5-minutes with $0.1 \mathrm{M}$ PBS. Free aldehyde groups were quenched with $0.3 \mathrm{M}$ glycine in $0.1 \mathrm{M}$ PBS at a $\mathrm{pH}$ of 7.4 followed by washing 2 times for 5-minutes with $0.1 \mathrm{M}$ PBS. Cells were then permeabilized with $0.025 \%$ Triton X-100 in PBS for 20-minutes and washed 3 times for 5-minutes with PBS. After 
permeabilization, cells were blocked with $2 \%$ BSA for 45 -minutes. Cells were then incubated with affinity purified IgG primary antibodies targeted against LC3 raised in rabbit (SigmaAldrich, St. Louis, MO) at a dilution of 1:500 in a solution containing 1\% BSA, 0.01\% Tween 20 in $0.1 \mathrm{M}$ PBS for 24-hours. After incubation with primary antibodies, cells were washed 3 times for 5-minutes with PBS containing $0.01 \%$ tween 20. Cells were then incubated with Alexa 488 secondary goat antibodies targeted against rabbit IgG (Invitrogen, Carlsbad, CA). Alexa 488 secondary antibodies were diluted to 1:1000 in a solution containing $10 \%$ goat serum, $0.01 \%$ Tween 20 in PBS and allowed to incubate with the cells for 4-hours followed by 3 times wash for 5 minutes. Cells were counterstained with DAPI nuclear stain (Molecular Probes, Eugene, OR) then mounted with a medium containing 3\% polyvinyl alcohol/glycerin/ Tris-HCl/ DABCO. Primary astrocytes were then imaged on a Zeiss LSM 710 violet confocal with a $63 \mathrm{X}$ magnification oil objective running on the Zen 2 platform. Confocal micrographs were analyzed for immunofluorescence colocalization of LC3 with MitoTracker labeled mitochondria as an indication of nascent mitophagy. LC3-MitoTracker colocalization was used to calculate the percentage of mitochondria in each mitochondrial size range undergoing mitophagy. Confocal micrographs were also used to calculate immunofluorescence intensity as a function of distance from the nucleus as an indication of LC3 localization.

\section{Mitochondrial ATP Production}

Mitochondrial ATP production was quantified using the CellTiter-Glo luminescent ATP assay kit (Promega, Madison WI) according to manufacturer's guidelines. Astrocytes were plated on 96-well black-walled assay plates and seeded at a density of 10,000 cells per well. After 24-hours of growth phase, cells received hypoxic treatment with or without reoxygenation and were allowed to equilibrate at room temperature for 30-minutes before the 
ATP assay was implemented. Luciferase luminescence was measured with a plate reader (BioTek). ATP was calculated in ng per 10,000 cells using an ATP standard curve. The standard curve was created immediately prior to running the assay to prevent ATP degradation. Standard curve was prepared by serial tenfold dilutions of ATP in DMEM.

\section{Mitochondrial Superoxide Production}

Time-dependent pre- and post-hypoxic reoxygenation associated superoxide $\left(\mathrm{O}_{2}^{\bar{z}}\right)$ production by mitochondria was measured with MitoSOX Red (Invitrogen), in accordance to manufacturer's guidelines. Measurements of $\mathrm{O}_{2}^{-}$were recorded at 3-hours before hypoxic exposure and $0,0.5,1,2,4,8$, and 12-hours post-hypoxia, during the reoxygenation period. Primary astrocytes were plated on 96-well assay plates containing DMEM at a seeding density of 15,000 cells per well. Astrocytes were then cultured to $95-100 \%$ confluency before experimentation. 5-minutes prior to receiving MitoSOX Red, astrocyte DMEM media was replaced with HBSS media to conduct the assay. Each well containing astrocytes received $5 \mu \mathrm{M}$ MitoSOX Red and allowed to incubate at $37^{\circ}$ for 16 -minutes. Fluorescence indication of $\mathrm{O}_{2}^{\bar{z}}$ levels were detected via an excitation of $510 \mathrm{~nm}$ and emission of $580 \mathrm{~nm}$ with a plate reader (BioTek). Fluorescence intensity was detected by the plate reader and computed into mean fluorescence values, these values were then used to calculate the percentage change from normoxia for each time point.

\section{Statistics}

All data were obtained using cells from at least four different animals/preparations. Results are depicted as means +/- SEM for the number of determinants (n) for each of the three experimental groups (normoxia, 3-hours hypoxia, and 3-hours hypoxia then 10-hours 
reoxygenation). Statistical analysis used was either student's t-test or ANOVA, with Bonferroni or Dunnett post-hoc tests. Specific statistical analysis used in each experiment is noted in the individual figure legends. Bonferroni's multiple comparison analysis was used to compare all combinations of the three experimental groups. Dunnett's post hoc test was used for the comparison of the experimental groups with the normoxia group (control group). P < 0.05 was considered significant. The following number of astrocytes' mitochondria were quantified: 7 normoxic astrocytes, 6 hypoxic astrocytes, and 6 hypoxic-reoxygenation astrocytes. For LC3 colocalization studies, the following number of astrocytes' mitochondria were quantified: 7 normoxic astrocytes, 5 hypoxic astrocytes, and 6 hypoxic-reoxygenation astrocytes.

\section{Results}

\section{Mitochondrial Size Measurements}

Astrocytic exposure to hypoxia demonstrated the dynamic ability of mitochondria to undergo fission and fusion in response to changes in environmental oxygen pressure (Figure 2.1A and B). Hypoxic exposure for 3-hours with or without 10-hours reoxygenation resulted in a redistribution of mitochondrial size to a larger number of smaller mitochondria.

Primary astrocytes that were exposed to 3-hours hypoxia with no reoxygenation showed the largest redistribution to smaller sized mitochondria compared to the 3-hours hypoxia then 10-hours reoxygenation and normoxia groups (Figure 2.1C). In the normoxia group, $35.86 \%$ of mitochondria fell within the $0.004-0.79 \mu \mathrm{m}^{2}$ range. After 3-hours of hypoxia these values increase to $64.59 \%(\mathrm{p}=0.0004)$ and after 10-hours of post-hypoxic reoxygenation the percentage of mitochondria that fell within the $0.004-0.79 \mu \mathrm{m}^{2}$ range 


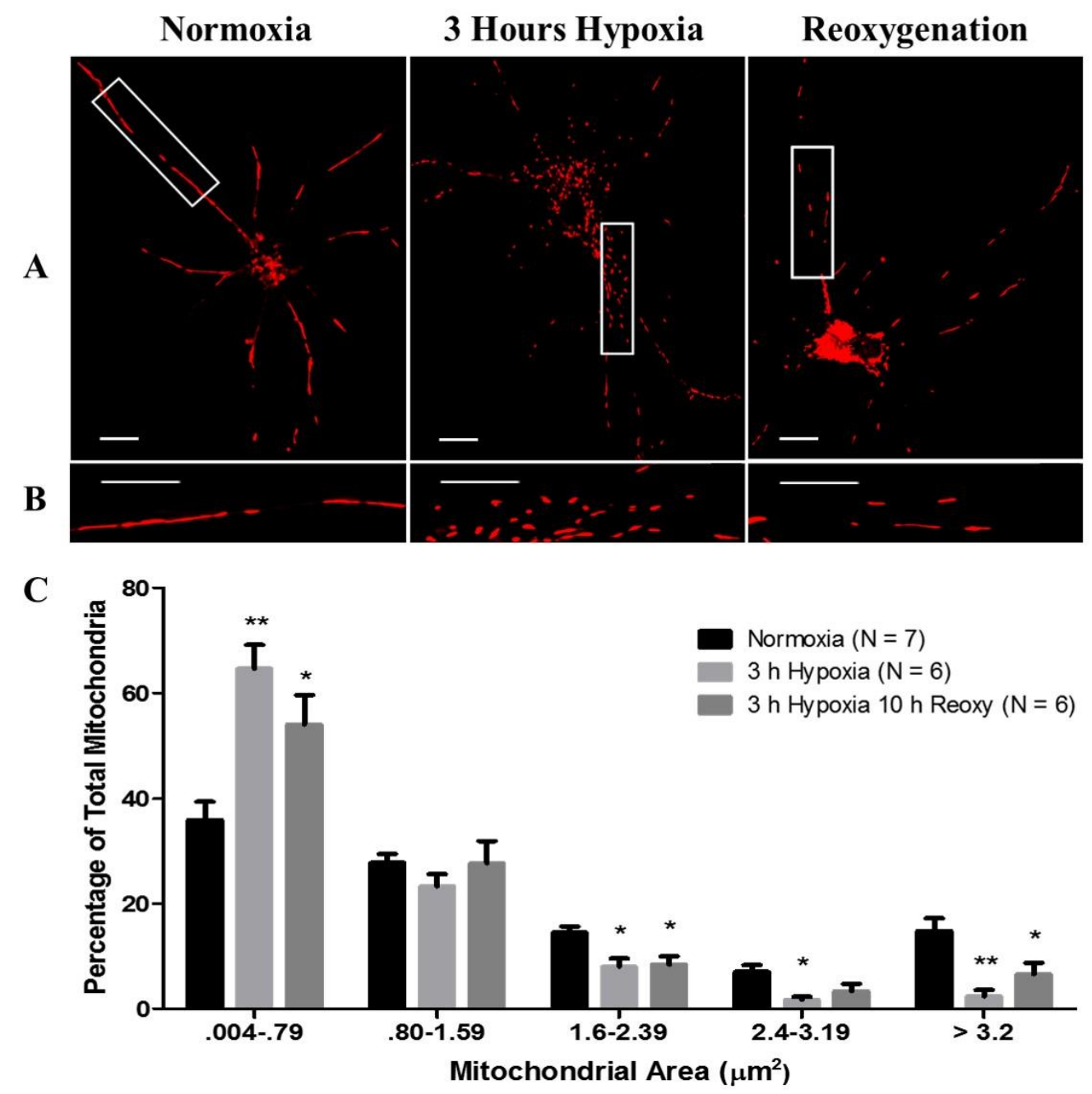

Figure 2.1. Hypoxic exposure induces excessive mitochondrial fission. (A and $B$ ) Micrographs depicting a 63X magnification of labeled mitochondria with MitoTracker Red CMXRos (Red) in primary rat astrocytes incubated under normoxia, 3-hours hypoxia and no reoxygenation, and 3-hours hypoxia then 10-hours reoxygenation. Row (B) contains magnified images of row (A). Scale bar $10 \mu \mathrm{m}$. Panel (C) contains a bar graph (mean $+/-$ SEM) depicting data of individual mitochondrial area $\left(\mu \mathrm{m}^{2}\right)$ as percent of the total mitochondria per astrocyte for each experimental group; normoxia $(n=550)$, 3-hours hypoxia $(\mathrm{n}=743)$, 3-hours hypoxia then 10-hours reoxygenation $(\mathrm{n}=312)$. Two-way ANOVA, Bonferroni's multiple comparison test was used to determine the level of significance between the experimental groups: $(*, p<0.05 ; * *, p<0.01)$. 
remained significantly elevated at $54.03 \%(\mathrm{p}=0.01)$ compared to normoxia. This observed increase in smaller mitochondria was associated with an observed reduction of larger mitochondria falling within $1.6-2.39,2.4-3.19$, and $>3.2 \mu \mathrm{m}^{2}$ range (Figure 2.1C). The average mitochondrial area differed between the groups; the normoxia group contained mitochondria with an average area of $1.69 \mu \mathrm{m}^{2}$ compared with $0.86 \mu \mathrm{m}^{2}(\mathrm{p}<0.01)$ and $1.08 \mu \mathrm{m}^{2}$ $(\mathrm{p}<0.05)$ for the 3-hours hypoxia and 3-hours hypoxia then 10-hours reoxygenation groups, respectively (Figure 2.2A). Mitochondrial swelling is a known phenomenon associated with calcium-induced cytochrome-c release from mitochondria during instances of mitochondrialinduced apoptosis (Kobayashi et al., 2003; Ichimura, 2011). To further make it clear that nascent cell death was absent during data collection and to determine if mitochondrial swelling contributes to the reported mitochondrial measurements, mitochondrial length and diameter were measured. Mitochondrial length was measured as the maximum distance between any two points within the mitochondrial boundaries. Mitochondrial diameter was measured as the minimum distance between two opposing points of mitochondrial boundaries. Astrocytes that were maintained under normoxia demonstrated a mean length of $2.70 \mu \mathrm{m}$ compared to 1.29 $\mu \mathrm{m}(\mathrm{p}<0.0001)$ and $2.05 \mu \mathrm{m}(\mathrm{p}<0.0001)$ after 3-hours of hypoxia and 10-hours post-hypoxic reoxygenation, respectively. Additionally, these data demonstrate a significant amelioration of mean mitochondrial length after 10-hours post-hypoxic reoxygenation $(\mathrm{p}<0.0001)$ compared to 3-hours hypoxia, albeit significantly reduced compared to normoxia (Figure 2.2B). In contrast to measurements of mitochondrial length, mitochondrial diameter did not depict any notable change between normoxia, 3-hours hypoxia, and 10-hours post-hypoxic reoxygenation (Figure 2.2C). 
Dephosphorylation of Drp-1 at Ser 637 by calcineurin is an essential step in mitochondrial fission (Cereghetti et al., 2008), and phosphorylation of Drp-1 at Ser 637 by PKA favors mitochondrial fusion (Chang and Blackstone, 2007; Cribbs and Strack, 2007). We therefore assessed Drp-1 phosphorylation at Ser 637 as percentage of total Drp-1 via Western blot analysis in primary astrocytes maintained under normoxia, 3-hours of hypoxia, and 10hours post-hypoxia reoxygenation. Our Western blot data revealed that astrocytes maintained under normoxic conditions contained $3.27 \%$ of total Drp-1 phosphorylated at Ser 637 whereas, astrocytes exposed to 3-hours of hypoxia contained 2.17\% ( $\mathrm{p}=0.0492)$ of Drp-1 phosphorylated at Ser 637 compared to normoxia. After 10-hours of post-hypoxia reoxygenation, Drp-1 phosphorylation at Ser 637 increased to $4.92 \%(\mathrm{p}=0.0058)$ of control Drp-1 phosphorylation (Figure 2.2D).

\section{Mitochondrial Population and Mass}

Interestingly, the total number of mitochondria per astrocyte was different among all three groups. In the normoxic control group, there were 79 mitochondria per astrocyte whereas the 3-hours hypoxia with no reoxygenation contained 124 mitochondria per astrocyte. However, after 10-hours of reoxygenation the number of mitochondria decreased to 52 per astrocyte $(\mathrm{p}=0.046)$ compared to normoxia (Figure 2.3A). To determine if the changes in mitochondrial population after hypoxia and reoxygenation affect the total mitochondrial content in astrocytes, we calculated the total mitochondrial area per astrocyte. Astrocytes that were maintained at normoxia contained a total mitochondrial area of $123 \mu \mathrm{m}^{2}$; however, following 3-hours of hypoxia the total mitochondrial area was reduced to $102 \mu \mathrm{m}^{2}$ and after 10-hours reoxygenation the total mitochondrial area was further reduced to $57 \mu \mathrm{m}^{2}(\mathrm{p}=0.0218)$ compared to normoxia (Figure 2.3B). 

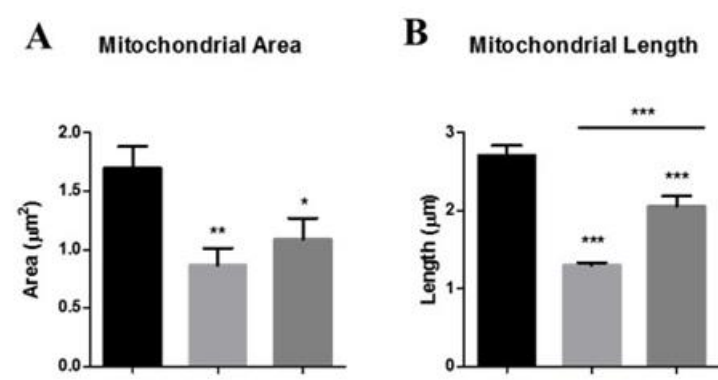

C Mitochondrial Diameter

D
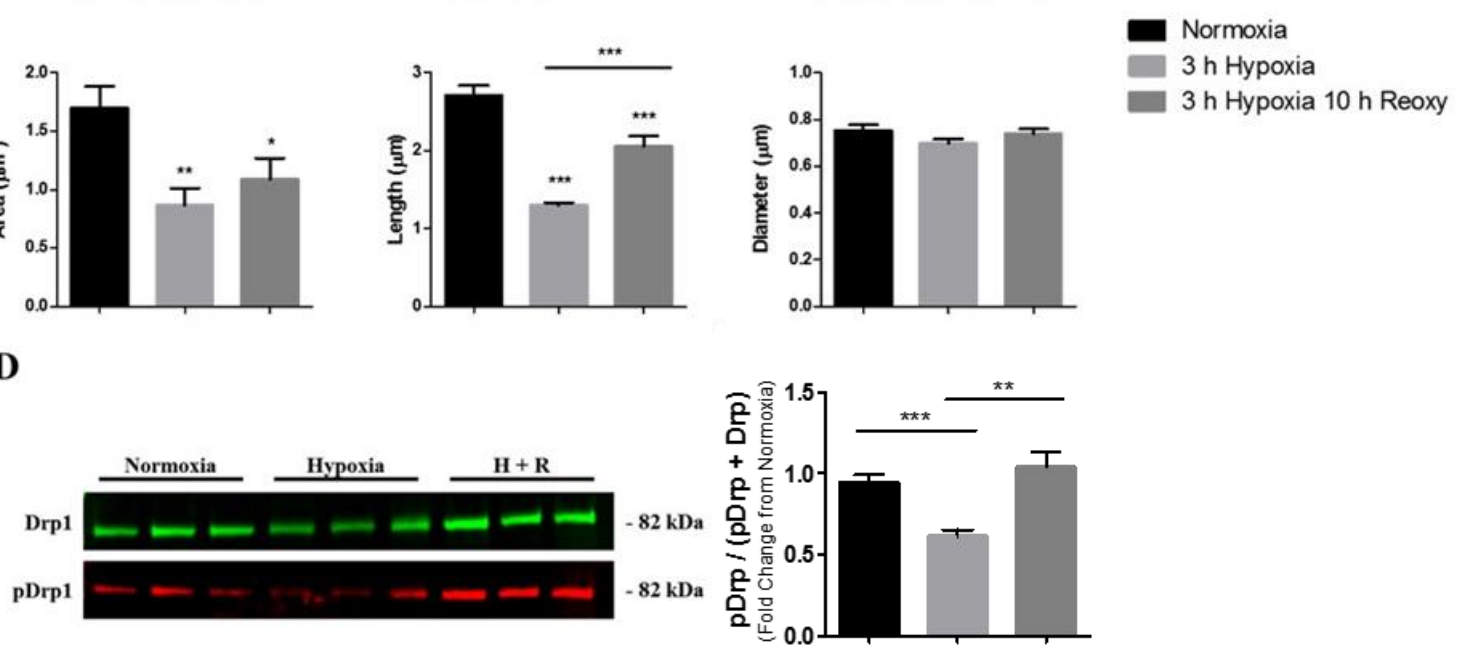

Figure 2.2. Measurements of mitochondria dimensions in astrocytes (A-C) after exposure to hypoxia and post-hypoxia reoxygenation. Bar graph (mean +/- SEM) depicting data of (A) mitochondrial area, (B) mitochondrial length, and (C) mitochondrial diameter as average of total mitochondria per astrocyte for each experimental group; normoxia $(\mathrm{n}=$ 550), 3-hours hypoxia $(\mathrm{n}=743), 3$-hours hypoxia then 10-hours reoxygenation $(\mathrm{n}=312)$.

(D) Characterization of Drp-1 phosphorylation after hypoxia and reoxygenation. SDSPAGE immunoblots indicate a reduced Drp-1 phosphorylation after hypoxic treatment and a subsequent increase with reoxygenation. Western blot analysis of whole cell lysates were determined by anti-pDrp-1 (Ser 637) antibodies (red) and anti-Drp-1 (green) for normoxia $(\mathrm{n}=11), 3$-hours hypoxia $(\mathrm{n}=14)$, and 3-hours hypoxia then 10 -hours reoxygenation $(\mathrm{H}+$ $\mathrm{R}, \mathrm{n}=16$ ). Bar graph (mean +/- SEM) depicting pDrp-1 data as the percentage of total Drp1. Panels A-C were analyzed with a one-way ANOVA, Bonferroni's multiple comparison test to determine the level of significance between the experimental groups $\left(^{*}, \mathrm{p}<0.05 ; * *\right.$, $\mathrm{p}<0.01 ; * * *, \mathrm{p}<0.0001)$. Panel D was analyzed with a one-tailed t-test to determine the level of significance between the experimental groups $(*, p<0.05 ; * *, p<0.01)$. 


\section{Mitochondrial Fragmentation}

Rounded mitochondria are indicative of fragmentation (Brustovetsky et al., 2009) and cellular stress (Dubinsky and Levi, 1998). Therefore, we measured mitochondrial roundness to assess the extent of mitochondrial fragmentation. These data are depicted on a scale from 0 to $1 ; 1$ being a perfect circle. Notably, astrocytes exposed to hypoxia displayed an alteration of mitochondrial morphology to a more rounded shape. Astrocytes that were maintained under normoxia contained mitochondria that scored an average of 0.36 on the roundness index. After 3-hours of hypoxia this score increased to $0.53(\mathrm{p}=0.0001)$ then decreased to $0.50(\mathrm{p}=0.0001)$ after 10-hours of reoxygenation (Figure 2.4A). A reduction of mitochondrial network complexity can result from fragmented mitochondria and increased mitochondrial fission. Therefore, mitochondrial aspect ratio (AR) and form factor (FF) was calculated to describe mitochondrial network complexity. Mitochondria that measure greater AR are larger mitochondria whereas mitochondria that score greater on FF contain more complex branching (Koopman et al., 2005a; b). Under normoxic conditions, mitochondria demonstrated an AR and FF of 4.49 and 2.58, respectively. After hypoxia exposure, the AR and FF decreased to 2.06 and 1.44, respectively indicating less complex mitochondrial networks. Furthermore, 10hours of post-hypoxic reoxygenation resulted in a slight amelioration of both AR (3.03) and FF (1.83) (Figure 2.4A-D).

\section{Mitochondrial Autophagy}

Excessive mitochondrial fragmentation may predispose mitochondria to loss of membrane potential and buffering capacity resulting in mitochondria being targeted for autophagy (Ishihara et al., 2003; Legros et al., 2003). Therefore, we next determined the extent of mitochondrial autophagy after exposure to hypoxia and post-hypoxia reoxygenation and 

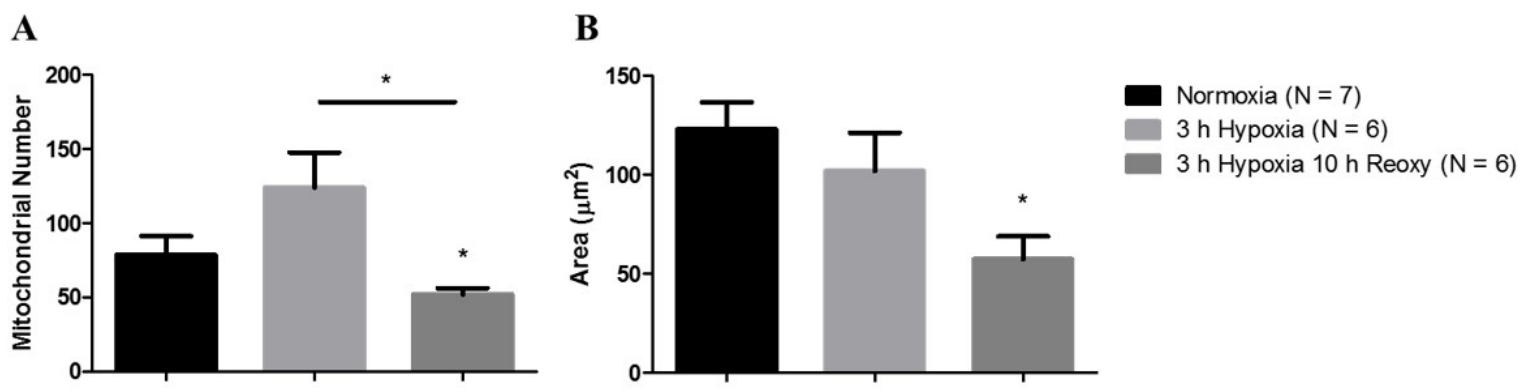

Figure 2.3. Astrocytic mitochondrial content fluctuates in response to hypoxia and reoxygenation. Bar graphs (mean +/- SEM) depicting the average total number of mitochondria per astrocyte $(A)$ and total area $\left(\mu \mathrm{m}^{2}\right)$ occupied by mitochondria per astrocyte (B) for each experimental group, normoxia (left, $n=7$ ), 3-hours hypoxia (center, $n=6$ ), and 3-hours hypoxia then 10-hours reoxygenation (right, $\mathrm{n}=6$ ). One-way ANOVA, Bonferroni's multiple comparison test was used to determine the level of significance between the experimental groups $(*, p<0.05)$. 
further to determine if smaller mitochondria are targeted for autophagy. During autophagy, cytosolic LC3 I is cleaved then lipidated before being inserted as LC3 II into the autophagosome membrane (Tanida et al., 2004). We first measured the conversion of LC3 I into LC3 II by calculating the ratio of LC3 II/ (LC3 I + LC3 II) (Mizushima and Yoshimori, 2007) via Western blot to determine the effects of hypoxia and post hypoxia reoxygenation on the number of autophagosomes. Astrocytes exposed to 3-hours hypoxia then 10-hours reoxygenation showed an RFU (relative fluorescence unit) of $0.436(\mathrm{p}<0.0001)$ conversion to LC3 II compared to 0.316 and 0.194 LC3 II conversion in the 3-hours hypoxia and normoxia groups, respectively (Figure 2.5A-B). Changes in LC3 II corresponded with a decrease in VDAC to $0.728(\mathrm{p}<0.02)$ and $0.803(\mathrm{p}<0.05)$ fold of normoxia in astrocytes exposed to 3hours hypoxia and 3-hours hypoxia then 10-hours reoxygenation, respectively (Figure 2.5C). We next performed an LC3 turnover assay to determine autophagic flux as an indication of autophagic induction (Mizushima and Yoshimori, 2007; Klionsky et al., 2008; Rubinsztein et al., 2009; Mizushima et al., 2010). The measurement of autophagic flux is centered on the notion that LC3 II is degraded in the autolysosome during autophagy. The LC3 turnover assay was performed by inhibiting lysosomal acidification with $30 \mu \mathrm{M}$ chloroquine prior to cells receiving hypoxic exposure. Astrocytes maintained at normoxia demonstrated an LC3 II turnover at 2.54 compared to $4.01(\mathrm{p}<0.006)$ and $7.91(\mathrm{p}<0.0001)$ in astrocytes exposed to 3hours hypoxia and 3-hours hypoxia then 10-hours reoxygenation, respectively (Figure 2.5D). In addition, under normoxic conditions, LC3 II was $204.04 \%(\mathrm{p}<0.0009)$ greater in astrocytes treated with $30 \mu \mathrm{M}$ chloroquine compared to astrocytes that were not treated with chloroquine (Figure 2.5E). These data demonstrating differences in LC3 II levels under basal conditions (normoxia) between astrocytes treated with chloroquine and without chloroquine rule out 


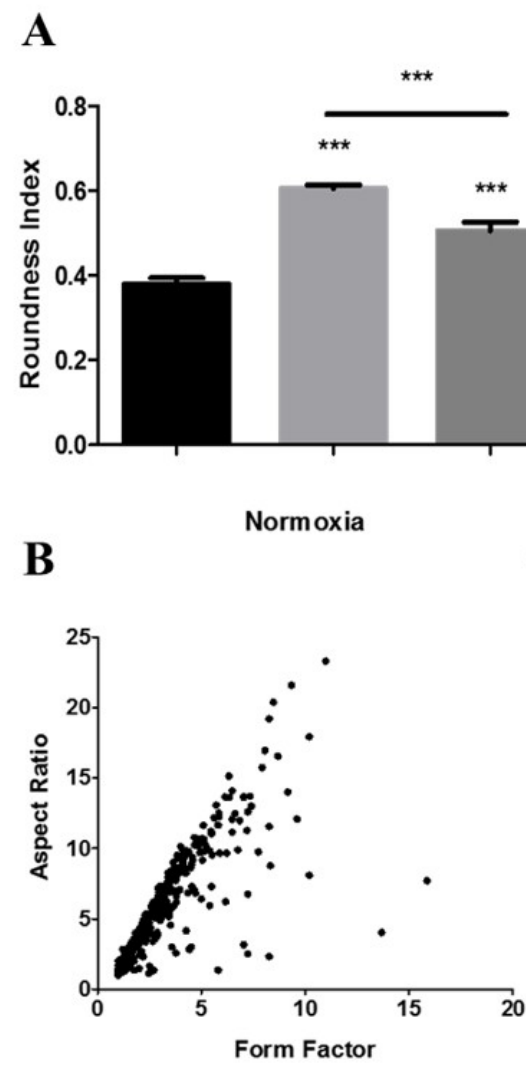

C

3 h Hypoxia

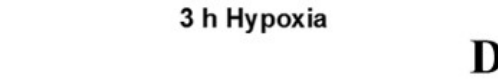

D 3 h Hypoxia $10 \mathrm{~h}$ Reoxy
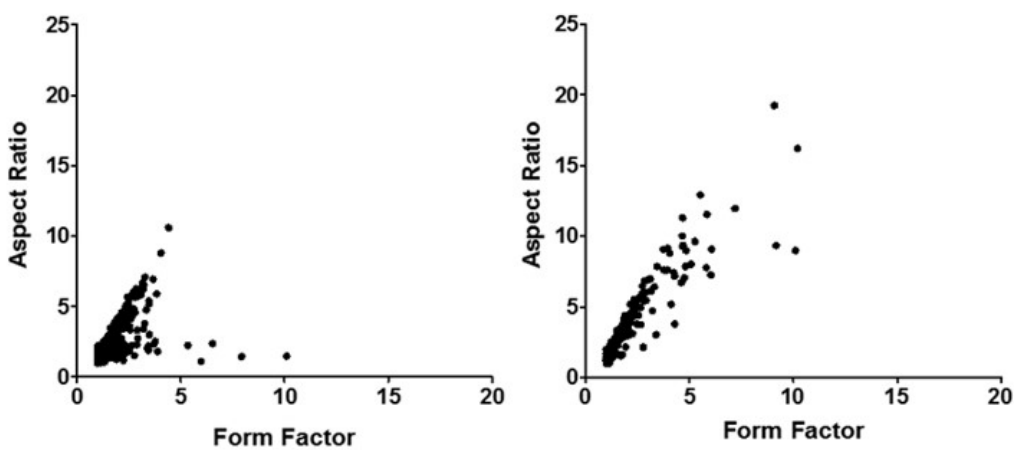

Figure 2.4. Hypoxia induces mitochondrial remodeling into a more rounded morphology and a loss of network complexity. (A) Bar graph depicting roundness values for each experimental group; normoxia (left, $\mathrm{n}=550$ ), 3-hours hypoxia (center, $\mathrm{n}=743$ ), and 3hours hypoxia then 10-hours reoxygenation (right, $\mathrm{n}=312$ ). (B-D) Scatter plots representing mitochondrial network complexity. Scatter plot of mitochondrial aspect ratio as a function of form factor for $(B)$ normoxia $(n=550),(C) 3$-hours hypoxia $(n=743)$, and (D) 3-hours hypoxia then 10-hours reoxygenation $(n=312)$. Increasing value of aspect ratio indicates larger size while increasing form factor value indicates greater mitochondrial network complexity. Panel A was analyzed with a one-way ANOVA, Bonferroni's multiple comparison test to determine the level of significance between experimental groups $(* * *$, $\mathrm{p}<0.0001)$. 
blocked autophagic processing as a contributor to LC3 II accumulation in our data (Figure $2.5 \mathrm{E})$.

Mitochondrial autophagy was assessed via immunofluorescence colocalization of LC3 and MitoTracker after hypoxia and post hypoxia reoxygenation. Confocal micrographs were collected from astrocytes maintained at normoxia, 3-hours hypoxia, and 10-hours post-hypoxic reoxygenation. As a positive control group, astrocytes maintained under normoxia were treated with 10uM FCCP to induce mitochondrial autophagy (Figure 2.5F). The confocal micrographs were used to create colormap representative images demonstrating colocalized mitochondria with LC3. The colormap images were used to calculate the percentage of mitochondria targeted for autophagy (Figure 2.5G), the size of mitochondria primarily affected by mitophagy (Figure $2.5 \mathrm{H}$ ), and the cellular localization of mitochondrial autophagic activity (Figure 2.5I). Our colocalization analysis indicated that under normoxic conditions $26.4 \%$ of total mitochondria are targeted for mitophagy compared to $24.9 \%$ and $(\mathrm{p}<0.01) 43.7 \%$ of mitochondria in astrocytes incubated under hypoxia for 3-hours and 3-hours hypoxia then 10-hours reoxygenation, respectively. In the positive control group, astrocytes treated with FCCP and maintained under normoxia contained $56.1 \%$ of their total mitochondria targeted for mitophagy. Our data indicate that the smallest mitochondria ranging in size of $0.004-0.79 \mu \mathrm{m}^{2}$ were most targeted by mitophagy. Astrocytes maintained under normoxia contained $3.68 \%$ of the smallest mitochondria targeted for mitophagy. Whereas, astrocytes exposed to 3-hours of hypoxia contained $7.23 \%(\mathrm{p}<0.0001)$ of the smallest mitochondria targeted for mitophagy. After 10-hours post-hypoxic reoxygenation the percentage of smallest mitochondria targeted for mitophagy increased to $17.45 \%(\mathrm{p}<0.0001)$. Mitochondria falling within the size rage of $0.80-1.59 \mu \mathrm{m}^{2}$ contained the second largest number of mitochondria targeted for mitophagy; 
these mitochondria represented the second-to smallest mitochondria. Astrocytes maintained under normoxia contained $6.59 \%$ of small mitochondria targeted for mitophagy. Whereas, astrocytes exposed to 3-hours of hypoxia contained $5.63 \%$ of small mitochondria targeted for mitophagy. After 10-hours of post-hypoxic reoxygenation the percentage of small mitochondria targeted for mitophagy significantly increased to $12.65 \%(\mathrm{P}<0.05)$. Additionally, our data suggests that the majority of mitochondrial autophagic activity occurs at specific cellular locations during hypoxia and post-hypoxic reoxygenation. Astrocytes exposed to 3hours of hypoxia then 10-hours reoxygenation showed increased LC3 fluorescence intensity near the nucleus compared to the approximate homogenous distribution of LC3 fluorescence intensity in astrocytes exposed to 3-hours of hypoxia or normoxia.

\section{Mitochondrial ATP Production}

Hypoxia induces mitochondrial respiratory inhibition resulting in a rapid decline of astrocytic ATP production. Astrocytes maintained under normoxia contained 52.89ng of ATP per 10,000 cells. After 3-hours of hypoxic exposure astrocytic ATP content declined to 23.1ng $(\mathrm{p}<0.0001)$ per 10,000 cells. Interestingly, after 10 -hours of post-hypoxic reoxygenation, astrocytes demonstrated a significant amelioration of ATP content to 47.12ng per 10,000 $(\mathrm{p}<0.0001)$ although still significantly below ATP content of astrocytes maintained under normoxia $(\mathrm{p}<0.0001)$ (Figure 2.6A).

\section{Mitochondrial Superoxide Production}

Mitochondrial superoxide production is a major contributor of cellular damage via oxidation of lipids, proteins, and other biomolecules. Mitochondrial superoxide production occurs at complex I and III of the mitochondrial electron transport chain, with the majority of superoxide production occurring at complex I via reversal of electron transport during cerebral 

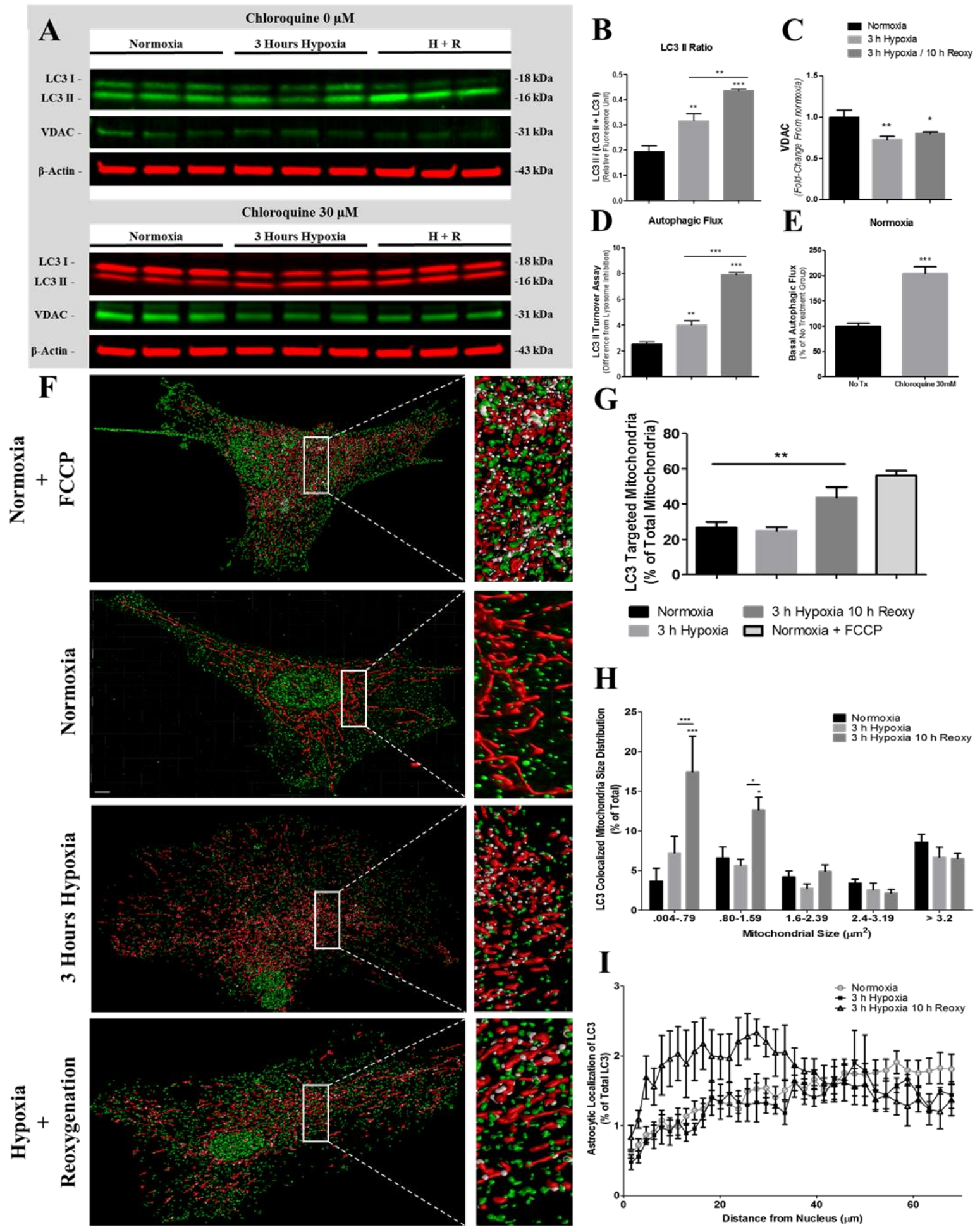

G

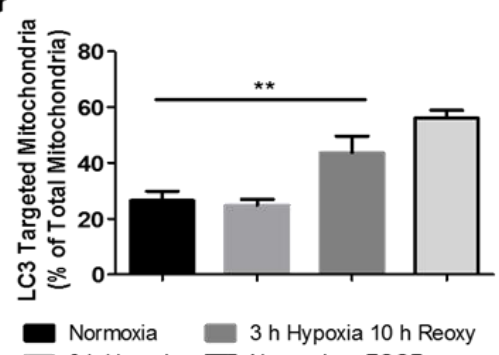

3 h Hypoxia $\square$ Normoxia + FCCP

H
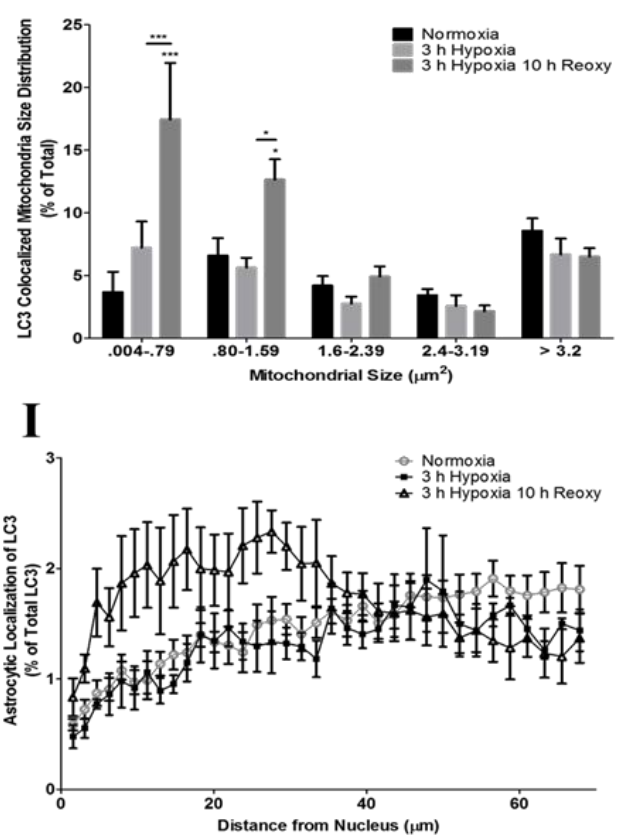
Figure 2.5. Expression and colocalization of LC3 with mitochondria in astrocytes. SDSPAGE immunoblots indicate an increased LC3 II content after hypoxia and reoxygenation (A) Western blot analysis of whole cell lysates were used to determine the magnitude of (B) LC3 II conversion, (C) cellular VDAC content, (D) autophagic flux, and (E) basal autophagic efficiency. Western blots of lysates from normoxia $(n=5)$, 3-hours hypoxia ( $n$ $=5)$, and 3-hours hypoxia then 10-hours reoxygenation $(\mathrm{H}+\mathrm{R}, \mathrm{n}=5)$ were labeled with anti-LC3 (red, green), VDAC (green), and $\beta$-actin (red) antibodies. (F) Representative three-dimensional reconstructions of z-stacks depicting mitochondria (red), LC3 (green), and colocalized areas (white) in astrocytes, incubated at normoxia (FCCP), normoxia, 3-

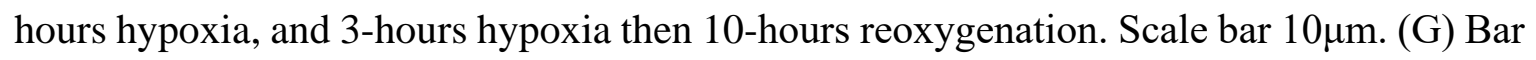
graph (mean +/- SEM) depicting the percentage of total mitochondria colocalized with LC3 in astrocytes incubated under normoxia $(\mathrm{FCCP})(\mathrm{n}=7)$, normoxia $(\mathrm{n}=7), 3$-hours hypoxia $(\mathrm{n}=5)$, and 3-hours of hypoxia then 10-hours reoxygenation $(\mathrm{n}=6)$. $(\mathrm{H})$ Bar graph $(+/-$ SEM) depicting percentage of mitochondria colocalized with LC3 as a function of mitochondrial size $\left(\mu \mathrm{m}^{2}\right)$. (I) Line graph depicting the percentage of extranuclear LC3 (+/SEM) as a function of nuclear distance for astrocytes incubated at normoxia (circle, $n=7$ ), 3-hours hypoxia (square, $\mathrm{n}=5$ ), and 3-hours hypoxia then 10-hours reoxygenation (triangle, $\mathrm{n}=6$ ). One-way ANOVA, Bonferroni's multiple comparison test was used to determine the level of significance between the experimental groups $(*, \mathrm{p}<0.05 ; * *, \mathrm{p}<0.01 ; * * *, \mathrm{p}$ $<0.0001)$. 
ischemia (Liu et al., 2002; Talbot et al., 2004). To determine the levels of superoxide generation by mitochondria, a fluorescence probe, MitoSOX Red, was used during normoxia, hypoxia, and subsequent time-periods of post-hypoxia reoxygenation. Our data indicates that astrocytes maintained under normoxia contained the highest levels of mitochondrial superoxide relative to hypoxia and post-hypoxia reoxygenation. At the conclusion of 3-hours hypoxic exposure, superoxide content reduced to $27.39 \%$ of the superoxide observed in normoxic astrocytes. After the first 30-minutes of post-hypoxic reoxygenation, astrocytic superoxide content increased to $56.71 \%$ of normoxic values. Furthermore, as post-hypoxic reoxygenation progressed, astrocytic superoxide production continued to increase to $60.72 \%$, $67.65 \%$, and $73.34 \%$ of normoxic superoxide content after 1-, 2, and 4-hours of reoxygenation, respectively. Interestingly, after 8-hours of post-hypoxic reoxygenation, astrocytic superoxide production reached a peak of $89.65 \%$ of normoxic values followed by a decline to $82.55 \%$ after 12-hours of post-hypoxic reoxygenation (Figure 2.6B).

\section{Astrocytic Extensions and Swelling}

Astrocytic extensions are structures that participate in critical cellular processes that mediate metabolic support to neurons, neurovascular coupling, and neurotransmitter recycling. Hypoxia and post-hypoxia reoxygenation induced a loss of astrocytic extensions. Astrocytes maintained under normoxia contained an average of 40 extensions. However, after exposure to 3-hours of hypoxia, astrocytic extensions declined to an average of 31 and after 10-hours of post-hypoxic reoxygenation, astrocytic extensions further declined to an average of 21 $(\mathrm{p}<0.05)$ (Figure 2.7A-D). We next determined the specific branch level of extension loss in these astrocytes. To determine the specific branch level of extension loss, each astrocytic 
A

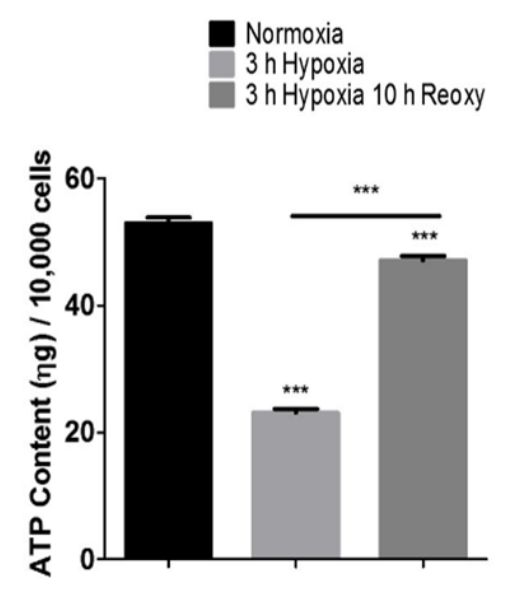

B

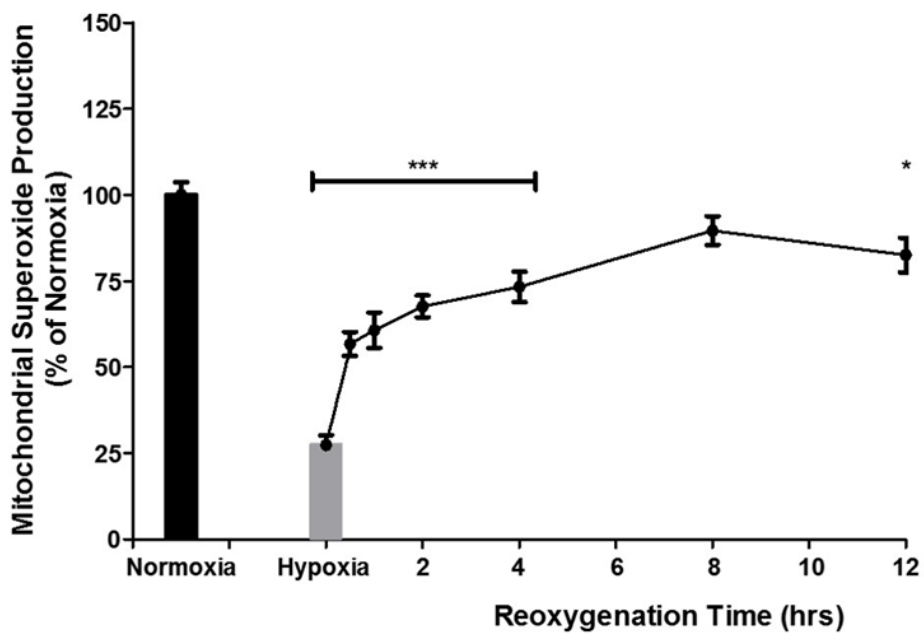

Figure 2.6. Bar graph (mean +/- SEM) depicting astrocytic ATP content (ng) per 10,000 cells for astrocytes incubated under normoxia $(n=18)$, 3-hours hypoxia $(n=12)$, and 3hours hypoxia then 10-hours reoxygenation $(\mathrm{n}=24)(\mathrm{A})$. Mitochondrial superoxide production during normoxia, after hypoxia and throughout post-hypoxia reoxygenation (B). Quasi bar graph and line graph (mean + - SEM) depicting the time-dependent superoxide production by mitochondria during normoxia $(\mathrm{n}=24)$, hypoxia $(\mathrm{n}=21)$, and for $0.5,1,2$, 4,8 , and 12-hours reoxygenation $(\mathrm{n}=22)$. Panel A was analyzed by a one-way ANOVA, Bonferroni's multiple comparison test was used to determine the level of significance between the experimental groups $(* * *, p<0.0001)$. Panel B was assessed by one-way ANOVA, Dunnett's post-hoc test was used to determine the level of significance between the experimental groups $(*, p<0.05 ; * * *, p<0.0001)$. 
extension was categorized based on branch origin. Extensions with origins of the soma were deemed primary extensions, extensions with origins on primary extensions were termed secondary extensions, and extensions with origins on secondary extensions were termed tertiary extensions. Our data revealed significant secondary and tertiary astrocyte extension loss. Astrocytes maintained under normoxia demonstrated the most complex branch patterning indicated by a higher number of secondary and tertiary extensions (Figure 2.7A and D). Normoxic astrocytes contained an average of 15 primary, 20 secondary, and 4 tertiary astrocytic extensions. However, after exposure to 3-hours of hypoxia, astrocytes demonstrated marked loss of extension density (Figure 2.7B and E). Astrocytes exposed to 3-hours of hypoxia contained 16 primary, 12 secondary, and 2 tertiary astrocytic extensions. After 10hours of post-hypoxic reoxygenation, astrocyte extension loss was clearly recognizable, in both density and complexity (Figure 2.7C and E). Astrocytes exposed to 3-hours hypoxia then 10hours reoxygenation contained 10 primary $(\mathrm{p}=0.0432), 8$ secondary $(\mathrm{p}=0.0063)$, and 0.5 tertiary ( $\mathrm{p}=0.0145$ ) astrocytic extensions (Figure $2.7 \mathrm{C}$ and E). Lastly, we observed evidence of astrocyte swelling during hypoxic exposure. This swelling did not persist throughout the reoxygenation phase of hypoxic exposure. Astrocytes maintained under normoxia contained an average astrocytic area of $1677.03 \mu \mathrm{m}^{2}$. However, after hypoxic incubation, astrocytic area significantly increased to $3076.01 \mu \mathrm{m}^{2}(\mathrm{p}=0.0425)$. Following 10-hours post-hypoxia reoxygenation, astrocytic area reduced to $1487.22 \mu \mathrm{m}^{2}$. These changes in astrocytic area indicate astrocyte swelling during hypoxic exposure that does not persist on to the reoxygenation phase of exposure (Figure 2.7F). Furthermore, our cell death assay of astrocytes exposed to 3-hours of hypoxia and 10-hours post-hypoxic reoxygenation (Figure 2.7G) 
indicated no change in astrocyte viability, suggesting that these observed changes in astrocytic swelling did not result in cellular death.

\section{Discussion}

In this study, we have demonstrated that hypoxia and post-hypoxia reoxygenation of primary astrocytes results in a redistribution of mitochondria to smaller sizes evoked by an increase in mitochondrial fission. Excessive mitochondrial fission corresponded to Drp-1 dephosphorylation at Ser 637, which preceded mitophagy of relatively small mitochondria. Post-hypoxia reoxygenation of astrocytes marked the initiation of elevated mitophagic activity particularly in the perinuclear region where a large number of the smallest mitochondria were undergoing nascent degradation. Although, during hypoxia astrocytic ATP content severely reduced, after reoxygenation ATP content returned to near normoxic values; these changes were observed in mitochondrial superoxide production as well. Concomitant with these mitochondrial morphological and functional changes in astrocytes, the number of astrocytic extensions declined particularly after 10-hours post-hypoxic reoxygenation. Overall, we posit a drastic mitochondrial network change that is triggered by a metabolic crisis during hypoxia; these changes are followed by mitochondrial degradation and retraction of astrocytic extensions.

Reports concerning mitochondrial fusion and fission as being beneficial or detrimental to cell function and viability have been considerably disparate (Chang and Blackstone, 2007; Estaquier and Arnoult, 2007; Parone et al., 2008; Ong et al., 2010; Gomes et al., 2011; Chou et al., 2012; Papanicolaou et al., 2012; Qi et al., 2013). However, it is well established that asymmetric mitochondrial fission is an important event central to mitochondrial quality control 


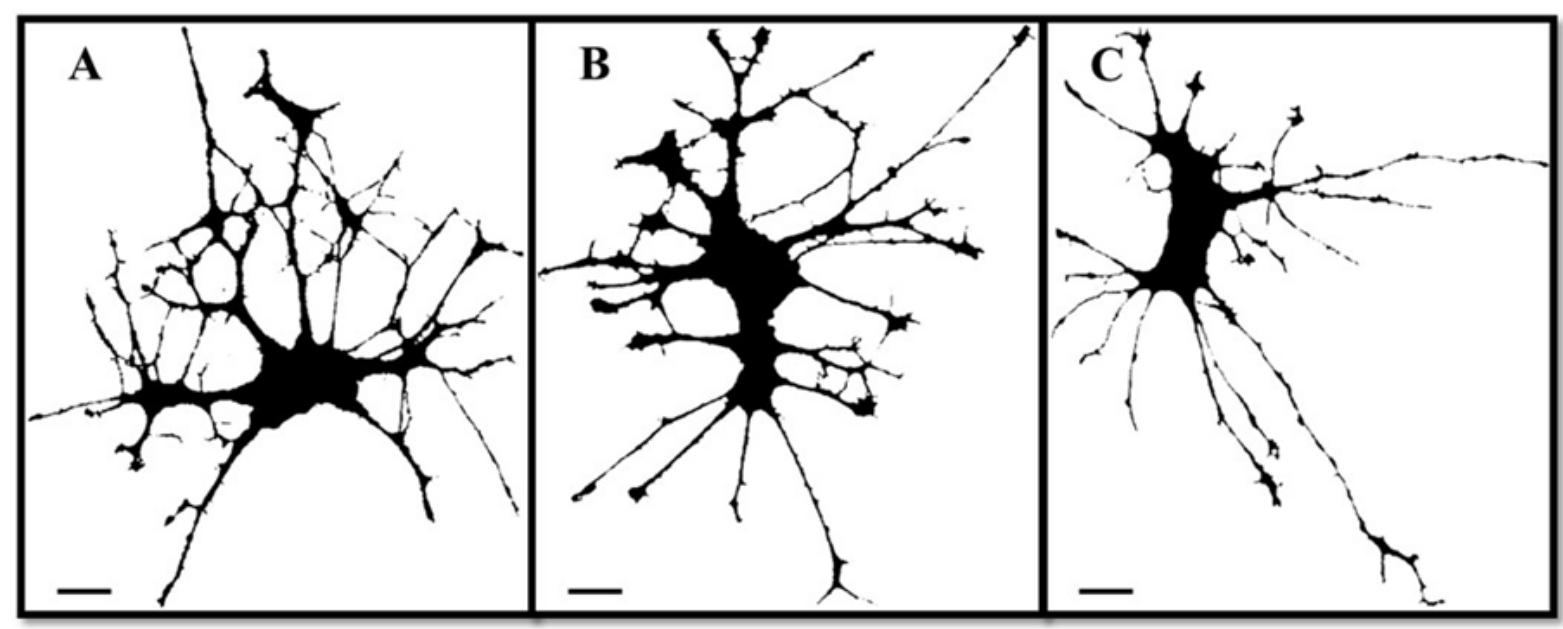

D

E

Astrocyte Extensions
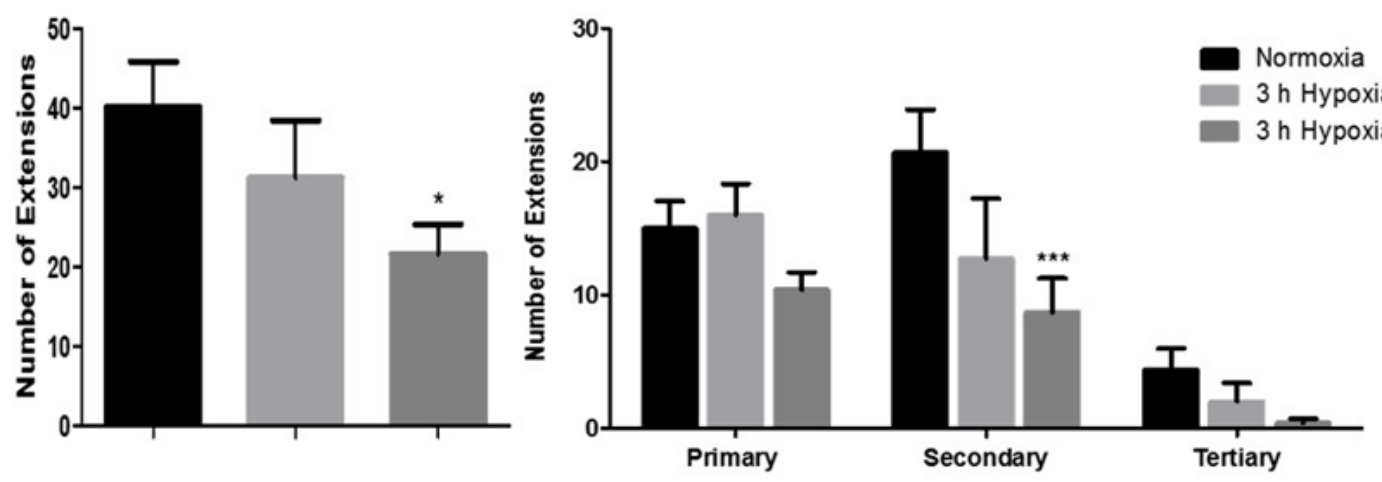

F

Astrocyte Area

G
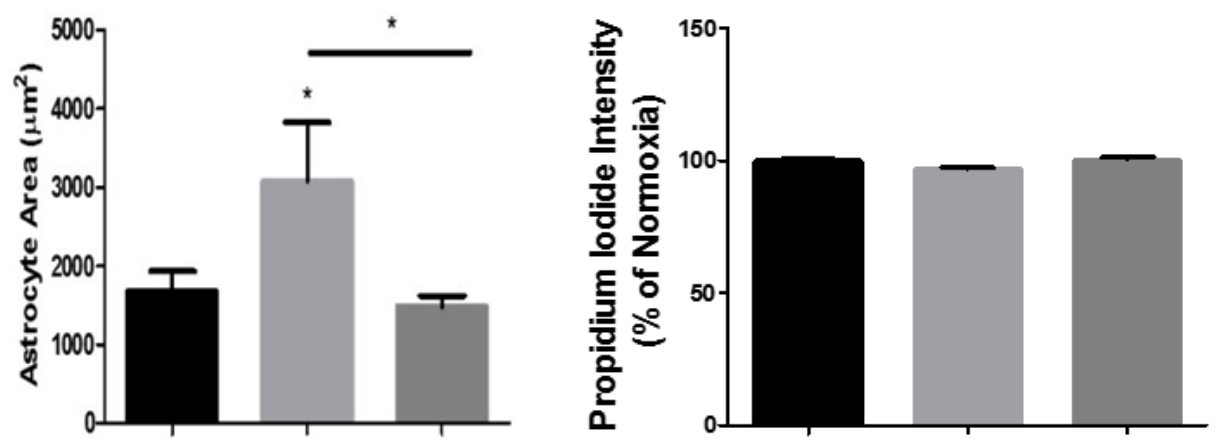
Figure 2.7. Hypoxic exposure and post-hypoxic reoxygenation results in a loss of astrocytic extensions. Confocal micrographs depicting $63 \mathrm{X}$ magnification binary images of primary astrocytes incubated under (A) normoxia $(n=7)$, (B) 3-hours hypoxia $(n=5)$, and (C) 3hours hypoxia then 10-hours reoxygenation $(n=7)$. Scale bar 10 $\mu$ m. (D) Bar graph (mean +/- SEM) depicting total number of astrocytic extensions for each experimental group. (E) Bar graph (mean +/- SEM) depicting the number of astrocytic extensions categorized into primary, secondary, or tertiary extensions per each experimental group. (F) Bar graph (mean + - SEM) depicting total astrocytic area $\left(\mu \mathrm{m}^{2}\right)$ for each experimental group normoxia $(\mathrm{n}=7)$, 3-hours hypoxia $(\mathrm{n}=6)$, 3-hours hypoxia then 10-hours reoxygenation $(\mathrm{n}=6)$. (G) Bar graph (mean $+/$ - SEM) depicting cell death indicated by Propidium iodide fluorescence intensity for each experimental group normoxia $(n=24)$, 3-hours hypoxia $(n=24)$, 3-hours hypoxia then 10-hours reoxygenation $(\mathrm{n}=24)$. One-way ANOVA, Bonferroni's multiple comparison test was used to determine the level of significance between the experimental groups $(* \mathrm{p}<0.05 ; * * *, \mathrm{p}<0.0001)$. 
that eliminates damaged or dysfunctional parts of mitochondria (Twig et al., 2008). We demonstrated that hypoxia results in a rapid redistribution of mitochondria to smaller sizes (Figure 2.1). Mitochondrial damage is an event of the reoxygenation phase of ischemic injury (Kowaltowski and Vercesi, 1999). Accordingly, our data indicated no increase in LC3 II conversion or elevations in LC3-targeted mitochondria in astrocytes maintained under hypoxia. In addition, the absence of mitophagic activity during hypoxia in astrocytes further suggests that the observed fission is independent of the organelle recycling that occurs during conditions of macromolecule depletion (Kristensen et al., 2008; Hailey et al., 2010; Egan et al., 2011). The most probable cause of mitochondrial fission during hypoxia is the onset of a metabolic crisis resulting from a rapid and severe ATP depletion. An inverse correlation between mitochondrial size and metabolic competence has been described in the rat brain (Bertoni-Freddari et al., 2003). This may explain our data suggesting that larger mitochondria predominantly undergo fission, increasing the number of smaller mitochondria during hypoxia and throughout reoxygenation. Therefore, we predict that during hypoxia an astrocytic metabolic crisis triggers mitochondrial fission as a functional means of increasing energy production by mitochondria.

It is well accepted that considerable cellular damage occurs during the reperfusion phase of ischemic injury (Kalogeris et al., 2012). A major contributor to ischemic-reperfusion injury is the mitochondrial production of superoxide at complex I (Arroyo et al., 1987; Bolli et al., 1989; Chouchani et al., 2013). Mitochondrial ROS production during initial ischemia reperfusion is driven by the rapid oxidation of succinate by the mitochondrial respiratory complex succinate dehydrogenase and reversal of electron transport (Chouchani et al., 2014). While this may devastate neuronal viability, astrocytes demonstrate increased tolerance to 
oxidative stress relative to neurons (Sochocka et al., 1994; Bolaños et al., 1995). Astrocyte tolerance to oxidative stress has been suggested to be an effect of greater metabolic plasticity (Escartin et al., 2007; Halim et al., 2010), more robust antioxidant buffering capacity (Dringen et al., 2000; Shih et al., 2003; Belanger and Magistretti, 2009), and a lesser dependence of ATP production via oxidative phosphorylation (Marrif and Juurlink, 1999; Solaini and Harris, 2005). Our data depict a progressive increase in mitochondrial superoxide production throughout the reoxygenation phase of astrocytes exposed to hypoxia, which occurred with no notable ROS burst. This gradual recovery of superoxide production parallels the oxidative phosphorylation rate of these mitochondria (Perez-Campo et al., 1998; Balaban et al., 2005). The absence of an ROS burst may explain astrocyte resistance to hypoxia-reoxygenationinduced cell death.

We provide evidence for two morphological responses of astrocytes to hypoxia. First, during hypoxia, we observed astrocytic swelling that did not persist throughout the reoxygenation phase. Astrocytic swelling is a known phenomenon of cerebral ischemia and possibly contributes to ischemic injury, including excitotoxicity, extracellular ionic dysregulation, and compressive damage to dendrites (Kimelberg et al., 1995; Kimelberg, 2004; Risher et al., 2012). This astrocytic swelling has been attributed to elevated cytosolic calcium concentration independent of mitochondrial depolarization (Kahlert and Reiser, 2002) in models of cerebral ischemia (Duffy and MacVicar, 1996; Brookes et al., 2004). The maintenance of homeostatic parameters of ion concentrations in astrocytes is dependent on ATP availability. Accordingly, our data show both a decline in astrocytic ATP content and swelling of astrocytes during hypoxia suggesting insufficient ATP availability for maintaining ionic homeostasis. Ultimately, astrocytic swelling and its regulatory mechanism render 
astrocytes depolarized (Kimelberg and O' Connor, 1988). This in mind, astrocyte morphology is clearly intimately coupled and acutely sensitive to the competence of cellular ATP production.

In addition to swelling, we also provide evidence for a robust retraction of secondary and tertiary astrocytic extensions after hypoxia-reoxygenation exposure. Astrocytic extensions are key structures enriched in excitatory amino acid transporters such as GLT-1, along with other surface receptors participating in homeostatic processes such as neurovascular coupling, translocation of specific molecules across the blood brain barrier, and maintenance of extracellular milieu.

During the energy crisis following ischemia, astrocytic ATP content is insufficient to support astrocyte relevant processes let alone fulfilling the energy requirement for astrocytemediated processes to neurons, which may account for some of the pathophysiological mechanism that degenerate neurons which are strongly thought to be prevented by astrocytic function.

A report by O’Donnell et al (O’Donnell et al., 2016) provided work consistent with our data in that, oxygen glucose deprivation (OGD) of hippocampal slice cultures resulted in a reduction of astrocytic mitochondrial length and occupancy (area). Furthermore, in agreement with our measurements of LC3 II (LC3 B) after hypoxia with no reoxygenation, O’Donnell and colleagues provide similar observations of a negligible change in LC3 B content with as much as 24-hours of OGD (O’Donnell et al., 2016). Importantly, our data depict a strong increase of LC3 B content occurring only with post-hypoxia reoxygenation suggesting that the bulk of mitochondrial autophagy occurs during the reoxygenation phase with hypoxic exposure. Therefore, pharmacological inhibition of mitochondrial autophagy in studies 
designed to investigate the effects of OGD on mitochondria occupancy would best be administered during a reoxygenation phase of OGD insult.

In summary, our data indicate a rapid metabolic crisis that ensues during hypoxia of astrocytes that is associated with reduced ATP production, a shift in mitochondrial distribution to smaller sizes, a loss of mitochondrial complexity, a reduction in mitochondrial superoxide production, and astrocytic swelling. During post-hypoxic-reoxygenation, there is a reduction in mitochondrial number mediated by smaller mitochondria targeted by mitophagy, a recovery of ATP production, restoration of mitochondrial superoxide production, and a general loss of astrocytic extensions.

\section{Acknowledgements}

This work was supported by NIH grants P20 GM109098, P01 AG027956, U54 GM104942 and T32 AG052375. Imaging experiments and image analysis were performed in the West Virginia University Microscope Imaging Facility, which has been supported by the Mary Babb

Randolph Cancer Center and NIH grants T32 AG052375, P20 RR016440, P30 RR032138/GM103488 and P20 RR016477.

\section{Author Contribution Statement}

DDQ designed studies, conducted studies and composed the manuscript. JAG, SNS, SJ, EBEC, AER, and JZC aided with studies, analyzed data, and revised the manuscript. JWS designed studies and revised the manuscript.

\section{Conflict of Interest}

The authors declare no competing financial interest. 


\section{References}

Almeida A, Medina JM. 1998. A rapid method for the isolation of metabolically active mitochondria from rat neurons and astrocytes in primary culture. Brain Res Protoc 2:209214.

Arroyo CM, Kramer JH, Dickens BF, Weglicki WB. 1987. Identification of free radicals in myocardial ischemia/reperfusion by spin trapping with nitrone DMPO. FEBS Lett 221:101-104.

Balaban RS, Nemoto S, Finkel T. 2005. Mitochondria, oxidants, and aging. Cell 120:483-495.

Belanger M, Magistretti PJ. 2009. The role of astroglia in neuroprotection. Dialogues Clin Neurosci 11:281-296.

Bertoni-Freddari C, Fattoretti P, Paoloni R, Caselli U, Giorgetti B, Solazzi M. 2003. Inverse correlation between mitochondrial size and metabolic competence: a quantitative cytochemical study of cytochrome oxidase activity. Naturwissenschaften 90:68-71.

Bolaños JP, Heales SJ, Land JM, Clark JB. 1995. Effect of peroxynitrite on the mitochondrial respiratory chain: differential susceptibility of neurones and astrocytes in primary culture. J Neurochem 64:1965-1972.

Bolli R, Jeroudi MO, Patel BS, DuBose CM, Lai EK, Roberts R, McCay PB. 1989. Direct evidence that oxygen-derived free radicals contribute to postischemic myocardial dysfunction in the intact dog. Proc Natl Acad Sci U S A 86:4695-4699.

Brookes PS, Yoon YS, Robotham JL, Anders MW, Sheu SS. 2004. Calcium, ATP, and ROS: a mitochondrial love-hate triangle. Am J Physiol Physiol 287:C817-C833.

Brustovetsky T, Li V, Brustovetsky N. 2009. Stimulation of glutamate receptors in cultured hippocampal neurons causes $\mathrm{Ca} 2+-$ dependent mitochondrial contraction. Cell Calcium 46:18-29.

Cereghetti GM, Stangherlin a, Martins de Brito O, Chang CR, Blackstone C, Bernardi P, Scorrano L. 2008. Dephosphorylation by calcineurin regulates translocation of Drp1 to mitochondria. Proc Natl Acad Sci U S A 105:15803-15808.

Chang C-R, Blackstone C. 2007. Cyclic AMP-dependent protein kinase phosphorylation of 
Drp1 regulates its GTPase activity and mitochondrial morphology. J Biol Chem 282:21583-7.

Chaudhry FA, Schmitz D, Reimer RJ, Larsson P, Gray AT, Nicoll RA, Kavanaugh M, Edwards RH. 2002. Glutamine uptake by neurons: interaction of protons with system a transporters. J Neurosci 22:62-72.

Chou CH, Lin CC, Yang MC, Wei CC, Liao H De, Lin RC, Tu WY, Kao TC, Hsu CM, Cheng JT, Chou AK, Lee CI, Loh JK, Howng SL, Hong YR. 2012. GSK3beta-Mediated Drp1 Phosphorylation Induced Elongated Mitochondrial Morphology against Oxidative Stress. PLoS One 7.

Chouchani ET, Methner C, Nadtochiy SM, Logan A, Pell VR, Ding S, James AM, Cochemé HM, Reinhold J, Lilley KS, Partridge L, Fearnley IM, Robinson AJ, Hartley RC, Smith RAJ, Krieg T, Brookes PS, Murphy MP. 2013. Cardioprotection by S-nitrosation of a cysteine switch on mitochondrial complex I. Nat Med 19:753-9.

Chouchani ET, Pell VR, Gaude E, Aksentijević D, Sundier SY, Robb EL, Logan A, Nadtochiy SM, Ord ENJ, Smith AC, Eyassu F, Shirley R, Hu C-H, Dare AJ, James AM, Rogatti S, Hartley RC, Eaton S, Costa ASH, Brookes PS, Davidson SM, Duchen MR, Saeb-Parsy K, Shattock MJ, Robinson AJ, Work LM, Frezza C, Krieg T, Murphy MP. 2014. Ischaemic accumulation of succinate controls reperfusion injury through mitochondrial ROS. Nature 515:431-435.

Cribbs JT, Strack S. 2007. Reversible phosphorylation of Drp1 by cyclic AMP-dependent protein kinase and calcineurin regulates mitochondrial fission and cell death. EMBO Rep 8:939-44.

Dringen R, Gutterer JM, Hirrlinger J. 2000. Glutathione metabolism in brain: Metabolic interaction between astrocytes and neurons in the defense against reactive oxygen species. Eur J Biochem 267:4912-4916.

Dubinsky JM, Levi Y. 1998. Calcium-induced activation of the mitochondrial permeability transition in hippocampal neurons. J Neurosci Res 53:728-741.

Duffy S, MacVicar B a. 1996. In vitro ischemia promotes calcium influx and intracellular calcium release in hippocampal astrocytes. J Neurosci 16:71-81. 
Egan DF, Shackelford DB, Mihaylova MM, Gelino S, Kohnz RA, Mair W, Vasquez DS, Joshi A, Gwinn DM, Taylor R, Asara JM, Fitzpatrick J, Dillin A, Viollet B, Kundu M, Hansen M, Shaw RJ. 2011. Phosphorylation of ULK1 (hATG1) by AMP-activated protein kinase connects energy sensing to mitophagy. Science 331:456-61.

Escartin C, Pierre K, Colin A, Brouillet E, Delzescaux T, Guillermier M, Dhenain M, Déglon N, Hantraye P, Pellerin L, Bonvento G. 2007. Activation of astrocytes by CNTF induces metabolic plasticity and increases resistance to metabolic insults. J Neurosci 27:70947104.

Estaquier J, Arnoult D. 2007. Inhibiting Drp1-mediated mitochondrial fission selectively prevents the release of cytochrome c during apoptosis. Cell Death Differ 14:1086-1094.

Gomes LC, Di Benedetto G, Scorrano L. 2011. During autophagy mitochondria elongate, are spared from degradation and sustain cell viability. Nat Cell Biol 13:589-98.

Hailey DW, Rambold AS, Satpute-Krishnan P, Mitra K, Sougrat R, Kim PK, LippincottSchwartz J. 2010. Mitochondria Supply Membranes for Autophagosome Biogenesis during Starvation. Cell 141:656-667.

Halim ND, Mcfate T, Mohyeldin A, Okagaki P, Korotchkina LG, Patel MS, Jeoung NH, Harris RA, Schell MJ, Verma A. 2010. Phosphorylation status of pyruvate dehydrogenase distinguishes metabolic phenotypes of cultured rat brain astrocytes and neurons. Glia 58:1168-1176.

Hansen AJ, Nedergaard M. 1988. Brain ion homeostasis in cerebral ischemia. Neurochem Pathol 9:195-209.

Ichimura T. 2011. Involvement of mitochondrial swelling in cytochrome c release from mitochondria treated with calcium and Alloxan. J Biophys Chem 2:10-18.

Ishihara N, Jofuku A, Eura Y, Mihara K. 2003. Regulation of mitochondrial morphology by membrane potential, and DRP1-dependent division and FZO1-dependent fusion reaction in mammalian cells. Biochem Biophys Res Commun 301:891-898.

Kahlert S, Reiser G. 2002. Swelling of mitochondria in cultured rat hippocampal astrocytes is induced by high cytosolic $\mathrm{Ca} 2+$ load, but not by mitochondrial depolarization. FEBS Lett 529:351-355. 
Kalogeris T, Baines CP, Krenz M, Korthuis RJ. 2012. Cell Biology of Ischemia/Reperfusion Injury.

Katayama Y, Kawamata T, Tamura T, Hovda DA, Becker DP, Tsubokawa T. 1991. Calciumdependent glutamate release concomitant with massive potassium flux during cerebral ischemia in vivo. Brain Res 558:136-140.

Kimelberg HK. 2004. Increased release of excitatory amino acids by the actions of ATP and peroxynitrite on volume-regulated anion channels (VRACs) in astrocytes. Neurochem Int 45:511-519.

Kimelberg HK, O' Connor E. 1988. Swelling of Astrocytes Causes Membrane Potential Depolarization. Glia:1219-224.

Kimelberg HK, Rutledge E, Goderie S, Charniga C. 1995. Astrocytic swelling due to hypotonic or high $\mathrm{K}+$ medium causes inhibition of glutamate and aspartate uptake and increases their release. J Cereb Blood Flow Metab 15:409-16.

Klionsky DJ, Abeliovich H, Agostinis P, Agrawal DK, Aliev G, Askew DS, Baba M, Baehrecke EH, Bahr BA, Ballabio A, Bamber BA, Bassham DC, Bergamini E, Bi X, Biard-Piechaczyk M, Blum JS, Bredesen DE, Brodsky JL, Brumell JH, Brunk UT, Bursch W, Camougrand N, Cebollero E, Cecconi F, Chen Y, Chin L-S, Choi A, Chu CT, Chung J, Clarke PGH, Clark RSB, Clarke SG, Clave C, Cleveland JL, Codogno P, Colombo MI, Coto-Montes A, Cregg JM, Cuervo AM, Debnath J, Demarchi F, Dennis PB, Dennis PA, Deretic V, Devenish RJ, Di Sano F, Dice JF, Difiglia M, Dinesh-Kumar S, Distelhorst CW, Djavaheri-Mergny M, Dorsey FC, Droge W, Dron M, Dunn WAJ, Duszenko M, Eissa NT, Elazar Z, Esclatine A, Eskelinen E-L, Fesus L, Finley KD, Fuentes JM, Fueyo J, Fujisaki K, Galliot B, Gao F-B, Gewirtz DA, Gibson SB, Gohla A, Goldberg AL, Gonzalez R, Gonzalez-Estevez C, Gorski S, Gottlieb RA, Haussinger D, He Y-W, Heidenreich K, Hill JA, Hoyer-Hansen M, Hu X, Huang W-P, Iwasaki A, Jaattela M, Jackson WT, Jiang X, Jin S, Johansen T, Jung JU, Kadowaki M, Kang C, Kelekar A, Kessel DH, Kiel JAKW, Kim HP, Kimchi A, Kinsella TJ, Kiselyov K, et al. 2008. Guidelines for the use and interpretation of assays for monitoring autophagy in higher eukaryotes. Autophagy 4:151-175.

Kobayashi T, Kuroda S, Tada M, Houkin K, Iwasaki Y, Abe H. 2003. Calcium-induced 
mitochondrial swelling and cytochrome $\mathrm{c}$ release in the brain: Its biochemical characteristics and implication in ischemic neuronal injury. Brain Res 960:62-70.

Kochanek KD, Murphy SL, Xu M.D. J, Arias Ph.D. E. 2014. Mortality in the United States, 2013. NCHS Data Brief; No 178:7.

Koopman WJH, Verkaart S, Visch HJ, van der Westhuizen FH, Murphy MP, van den Heuvel L, Smeitink JAM, Willems P. 2005a. Inhibition of complex I of the electron transport chain causes O-2(-)center dot-mediated mitochondrial outgrowth. Am J Physiol Physiol 288:C1440-C1450.

Koopman WJH, Visch H-J, Verkaart S, van den Heuvel LWPJ, Smeitink J a M, Willems PHGM. 2005b. Mitochondrial network complexity and pathological decrease in complex I activity are tightly correlated in isolated human complex I deficiency. Am J Physiol Cell Physiol 289:C881-C890.

Kowaltowski AJ, Vercesi AE. 1999. Mitochondrial damage induced by conditions of oxidative stress. Free Radic Biol Med 26:463-471.

Kristensen AR, Schandorff S, Høyer-Hansen M, Nielsen MO, Jäättelä M, Dengjel J, Andersen JS. 2008. Ordered organelle degradation during starvation-induced autophagy. Mol Cell Proteomics 7:2419-2428.

Legros F, Lombès A, Frachon P, Rojo M. 2003. Mitochondrial Fusion in Human Cells Is Efficient, Requires the Inner Membrane Potential, and Is Mediated by Mitofusins. Mol Biol Cell 14:2559-2569.

Liu Y, Fiskum G, Schubert D. 2002. Generation of reactive oxygen species by the mitochondrial electron transport chain. J Neurochem 80:780-787.

Lovatt D, Sonnewald U, Waagepetersen HS, Schousboe A, He W, Lin JH-C, Han X, Takano T, Wang S, Sim FJ, Goldman S a, Nedergaard M. 2007. The transcriptome and metabolic gene signature of protoplasmic astrocytes in the adult murine cortex. J Neurosci 27:12255-66.

Marrif H, Juurlink BHJ. 1999. Astrocytes respond to hypoxia by increasing glycolytic capacity. J Neurosci Res 57:255-260.

Mizushima N, Yoshimori T. 2007. How to interpret LC3 immunoblotting. Autophagy 3:542- 
545.

Mizushima N, Yoshimori T, Levine B. 2010. Methods in mammalian autophagy research. Cell 140:313-326.

Mozaffarian D, Benjamin EJ, Go AS, Arnett DK, Blaha MJ, Cushman M, de Ferranti S, Despres J-P, Fullerton HJ, Howard VJ, Huffman MD, Judd SE, Kissela BM, Lackland DT, Lichtman JH, Lisabeth LD, Liu S, Mackey RH, Matchar DB, McGuire DK, Mohler ER, Moy CS, Muntner P, Mussolino ME, Nasir K, Neumar RW, Nichol G, Palaniappan L, Pandey DK, Reeves MJ, Rodriguez CJ, Sorlie PD, Stein J, Towfighi A, Turan TN, Virani SS, Willey JZ, Woo D, Yeh RW, Turner MB. 2015. Heart Disease and Stroke Statistics--2015 Update: A Report From the American Heart Association.

Nehlig A, Wittendorp-Rechenmann E, Lam CD. 2004. Selective uptake of [14C]2deoxyglucose by neurons and astrocytes: high-resolution microautoradiographic imaging by cellular 14C-trajectography combined with immunohistochemistry. J Cereb Blood Flow Metab 24:1004-1014.

O’Donnell JC, Jackson JG, Robinson MB. 2016. Transient Oxygen/Glucose Deprivation Causes a Delayed Loss of Mitochondria and Increases Spontaneous Calcium Signaling in Astrocytic Processes. J Neurosci 36:7109-7127.

Ong SB, Subrayan S, Lim SY, Yellon DM, Davidson SM, Hausenloy DJ. 2010. Inhibiting mitochondrial fission protects the heart against ischemia/reperfusion injury. Circulation 121:2012-2022.

Papanicolaou KN, Ngoh G a., Dabkowski ER, O’Connell K a., Ribeiro RF, Stanley WC, Walsh K. 2012. Cardiomyocyte deletion of mitofusin-1 leads to mitochondrial fragmentation and improves tolerance to ROS-induced mitochondrial dysfunction and cell death. AJP Hear Circ Physiol 302:H167-H179.

Parone PA, Da Druz S, Tondera D, Mattenberger Y, James DI, Maechler P, Barja F, Martinou JC. 2008. Preventing mitochondrial fission impairs mitochondrial function and leads to loss of mitochondrial DNA. PLoS One 3.

Parpura V, Haydon PG. 2000. Physiological astrocytic calcium levels stimulate glutamate release to modulate adjacent neurons. Proc Natl Acad Sci U S A 97:8629-8634. 
Paulsen RE, Fonnum F. 1989. Role of glial cells for the basal and Ca2+-dependent K+-evoked release of transmitter amino acids investigated by microdialysis. J Neurochem 52:18231829.

Perez-Campo R, L??pez-Torres M, Cadenas S, Rojas C, Barja G. 1998. The rate of free radical production as a determinant of the rate of aging: Evidence from the comparative approach. J Comp Physiol - B Biochem Syst Environ Physiol 168:149-158.

Qi X, Qvit N, Su YC, Mochly-Rosen D. 2013. A novel Drp1 inhibitor diminishes aberrant mitochondrial fission and neurotoxicity. J Cell Sci 126:789-802.

Risher WC, Croom D, Kirov SA. 2012. Persistent astroglial swelling accompanies rapid reversible dendritic injury during stroke-induced spreading depolarizations. Glia 60:1709-1720.

Rossi DJ, Brady JD, Mohr C. 2007. Astrocyte metabolism and signaling during brain ischemia. Nat Neurosci 10:1377-1386.

Rothstein JD, Dykes-Hoberg M, Pardo CA, Bristol LA, Jin L, Kuncl RW, Kanai Y, Hediger MA, Wang Y, Schielke JP, Welty DF. 1996. Knockout of glutamate transporters reveals a major role for astroglial transport in excitotoxicity and clearance of glutamate. Neuron 16:675-686.

Rubinsztein DC, Cuervo AM, Ravikumar B, Sarkar S, Korolchuk V, Kaushik S, Klionsky DJ. 2009. In search of an "autophagomometer". Autophagy 5:585-589.

Shih AY, Johnson DA, Wong G, Kraft AD, Jiang L, Erb H, Johnson JA, Murphy TH. 2003. Coordinate regulation of glutathione biosynthesis and release by Nrf2-expressing glia potently protects neurons from oxidative stress. J Neurosci 23:3394-3406.

Silver IA, Deas J, Erecińska M. 1997. Ion homeostasis in brain cells: Differences in intracellular ion responses to energy limitation between cultured neurons and glial cells. Neuroscience 78:589-601.

Sochocka E, Juurlink BHJ, Code WE, Hertz V, Peng L, Hertz L. 1994. Cell death in primary cultures of mouse neurons and astrocytes during exposure to and "recovery" from hypoxia, substrate deprivation and simulated ischemia. Brain Res 638:21-28.

Solaini G, Harris D a. 2005. Biochemical dysfunction in heart mitochondria exposed to 
ischaemia and reperfusion. Biochem J 390:377-394.

Swanson RA, Shiraishi K, Morton MT, Sharp FR. 1990. Methionine sulfoximine reduces cortical infarct size in rats after middle cerebral artery occlusion. Stroke 21:322-327.

Talbot DA, Lambert AJ, Brand MD. 2004. Production of endogenous matrix superoxide from mitochondrial complex I leads to activation of uncoupling protein 3. FEBS Lett 556:111115.

Tanida I, Ueno T, Kominami E. 2004. LC3 conjugation system in mammalian autophagy. Int J Biochem Cell Biol 36:2503-2518.

Twig G, Elorza A, Molina AJA, Mohamed H, Wikstrom JD, Walzer G, Stiles L, Haigh SE, Katz S, Las G, Alroy J, Wu M, Py BF, Yuan J, Deeney JT, Corkey BE, Shirihai OS. 2008. Fission and selective fusion govern mitochondrial segregation and elimination by autophagy. EMBO J 27:433-446.

Waniewski R a, Martin DL. 1986. Exogenous glutamate is metabolized to glutamine and exported by rat primary astrocyte cultures. J Neurochem 47:304-13. 


\title{
CHAPTER 3
}

\section{Gradual Common Carotid Artery Occlusion as a Novel Model for Cerebrovascular Hypoperfusion}

\author{
Dominic D. Quintana ${ }^{1}$, Xuefang Ren ${ }^{2,3,5}$, Heng Hu ${ }^{1,5}$, Elizabeth B. Engler-Chiurazzi ${ }^{1,2}$, \\ Stephanie L. Rellick ${ }^{1}$, Sara E. Lewis ${ }^{1}$, Jessica M. Povroznik ${ }^{1}$, James W. Simpkins ${ }^{1,5}$, and \\ Mohammad Alvi
}

\author{
${ }^{1}$ Department of Physiology and Pharmacology, \\ ${ }^{2}$ Department of Neuroscience, \\ ${ }^{3}$ Department of Microbiology, Immunology \& Cell Biology, \\ ${ }^{4}$ Department of Neurology, \\ ${ }^{5}$ Experimental Stroke Core, \\ Center for Basic and Translational Stroke Research \\ West Virginia University, Morgantown, West Virginia, 26506 USA
}

\section{Author Contribution Statement}

DDQ designed studies, conducted studies and composed the manuscript. XR Designed studies and revised manuscript. HH, EBEC, SLR, SEL, and JMP conducted studies. JWS and MA designed studies and revised the manuscript. 


\begin{abstract}
Chronic cerebrovascular hypoperfusion results in vascular dementia and increases predisposition to lacunar infarcts. However, there are no suitable animal models. In this study, we developed a novel model for chronic irreversible cerebral hypoperfusion in mice. Briefly, an ameroid constrictor was placed on the right carotid artery to gradually occlude the vessel, while a microcoil was placed on the left carotid artery to prevent compensation of the blood flow. This procedure resulted in a gradual hypoperfusion developing over a period of 34 days with no cerebral blood flow recovery. Histological analysis of the brain revealed neuronal and axonal degeneration as well as necrotic lesions. The most severely affected regions were located in the hippocampus and the corpus callosum. Overall, our paradigm is a viable model to study brain pathology resulting from gradual cerebrovascular hypoperfusion.
\end{abstract}


Keywords: White matter pathology, hypoperfusion, small vessel disease, gradual vessel occlusion, cerebral blood flow, neuronal degeneration 


\section{Introduction}

Chronic cerebral hypoperfusion is the primary cause of vascular dementia (Kynast et al., 2017), and has been implicated in the development of white matter disease and lacunar infarcts (Black et al., 2009). During the progression of the disease, cerebral arteries harden, resulting in a deficient nutrient delivery to the cerebral parenchyma. This in turn, causes metabolic distress and bioenergetic disturbances that contribute to cerebral degeneration (Vasquez and Zakzanis, 2015). There are several models to induce hypoperfusion in experimental animals based on the occlusion of two (Bottiger et al., 1998; Bottiger et al., 1999), three (Carmichael, 2005; Thal et al., 2010; Onken et al., 2012) or four (Pulsinelli and Buchan, 1988; Traystman, 2003) vessels. However, all these models are based on either permanent or temporal reduction/blockade of blood flow. Therefore, there is a need for a more clinically relevant model that would induce a gradual reduction of cerebral blood flow, and therefore, simulate chronic cerebral hypoperfusion in humans.

In this study, we developed a novel murine model to gradually, and irreversibly reduce cerebral blood perfusion over time. Our paradigm is advantageous because it circumvents the hypoxic/ischemic damage associated with a rapid reduction in CBF observed in other hypoperfusion models (Shibata et al., 2004; Kitamura et al., 2016). 


\section{Materials and Methods}

\section{Animals}

12-14 week-old male C57BL/6Jmice were used. Mice were housed in accordance to Institutional Animal Care and Use Committee (IACUC) guidelines in the West Virginia University (WVU) Health Sciences Center vivarium. Animals were maintained under a light/dark cycle (12:12 h) with food and water available ad libitum. All procedures conducted were approved by IACUC at WVU.

\section{Implantation of Ameroid Constrictor Ring and Microcoil}

The ameroid constrictor is a device used in veterinary medicine for the treatment of hepatic shunts whereby it induces collateral circulation via blood vessel occlusion. The constrictor ring is composed of a surgical steel ring and an inner layer composed of casein. The hygroscopic property of casein causes its gradual swelling at a predictable rate, wherein the surgical steel ring that surrounds the casein layer forces this swelling inward, resulting in a shrinking inner diameter that gradually occludes blood vessels.

Mice were anesthetized with 4-5\% isoflurane and maintained under 1-2\% isoflurane in a $30 \% \mathrm{O}_{2}: 70 \% \mathrm{~N}_{2}$ mixture and placed on a feedback controlled heating pad to maintain the body temperature at $37^{\circ} \mathrm{C}$. Ophthalmic ointment was placed on the eyes; the surgical area was prepared by trimming the fur and sanitizing the skin with isopropanol pads followed by betadine. Both common carotid arteries (CCAs) were exposed through a midline cervical incision, and an ameroid constrictor ring (cat. MC-0.50-55, Research Instruments SW, CA) was placed around the right CCA following a published protocol (Hattori et al., 2015). A 
microcoil (cat. SWPA ID 0.18, Wuxi Samini Spring Co., LTD, China) was placed around the left CCA to prevent CBF compensation. The entire surgical procedure was completed within 20 minutes. The sham group received a sham surgery including an incision exposing the common carotid arteries but did not receive the implantation of the ameroid constrictor ring and placement of the microcoil. All further experimentation was performed blinded to surgical group.

\section{Cerebral Blood Flow Measurement}

Before the placement of the ameroid constrictor ring and microcoil, baseline CBF was measured. Briefly, the skull was exposed with a $1.5 \mathrm{~cm}$ incision. Ten consecutive measurements of $\mathrm{CBF}$ were acquired with a MoorFLPI laser Doppler system (Moor Instruments, England) over the course of 5 minutes with an exposure of $200 \mathrm{~ms}$. The incision was closed with sutures and the animals were subcutaneously injected with a local anesthetic, bupivacaine $(1 \mathrm{mg} / \mathrm{kg})$, once a day for 3 days. Implantation surgery was performed (see above). To make certain that the implantation surgery did not cause damage to the CAA, a second CBF measurement was performed. Subsequent CBF measurements were performed by reopening the scalp at the same incision site, followed by suturing and bupivacaine injections.

\section{Histochemistry}

On day 34, mice were anesthetized and transcardially perfused with $20 \mathrm{~mL} 0.01 \mathrm{M}$ phosphate buffered saline followed by $20 \mathrm{~mL}$ of $4 \%$ paraformaldehyde, $\mathrm{pH}$ of 7.45 . Brains were extracted, placed in $4 \%$ paraformaldehyde and incubated at $4{ }^{\circ} \mathrm{C}$ overnight. Fixed brains 
were sliced into $2 \mathrm{~mm}$ coronal sections, embedded in paraffin, sliced into $10 \mu \mathrm{m}$ coronal sections and mounted onto specimen slides. Deparaffinized and rehydrated tissue sections were stained with Haemotoxylin and Eosin (H\&E) to assess general brain pathology (Xiong et al., 2008) and silver stained to assess axonal damage (Uchihara, 2007). The sections were imaged on a MIF Olympus VS120 Slide Scanner at 20X magnification.

\section{Statistical Analysis}

Statistical comparisons were performed using either Student's t-test or ANOVA (with or without repeated measures, where appropriate). Dunnett's post-hoc test was used for comparison of the experimental groups relative to a control group, or for comparison within a given group at one time point post-surgery (sham or surgical hypoperfusion) relative to the pre-surgery time point. $\mathrm{p}<0.05$ was considered significant.

\section{Results}

\section{Cerebral Blood Flow}

Out of the initial seven mice in the hypoperfusion group, two died on day 15 and 17, post-surgery. There was no mortality in the sham group. The impact of surgical hypoperfusion was assessed by measuring changes in global CBF over time (Figure 3.1). To account for group differences in pre-hypoperfusion CBF values, we calculated the percent change at each experimental time point (day 1,3,7,13,27, and 34) relative to the average of the values at the pre-surgery time point within each surgical intervention group individually (sham or hypoperfusion) (Figure 3.1B). One sham animal was excluded from the analyses due to 
inaccurate $\mathrm{CBF}$ measurements at the pre-surgery time point. Using these values, we conducted repeated measures ANOVA with surgical intervention as the independent factor and time point as the repeated factor. There was a significant interaction between these factors $[F(6,63)=$ 4.967, $p<0.0005]$. To determine the extent to which our surgical hypoperfusion model induced a gradual constriction that progressively reduced $\mathrm{CBF}$, we probed the significant interaction by evaluating temporal $\mathrm{CBF}$ changes within each surgical intervention group separately using the repeated measures ANOVA where time was the repeated factor. For the sham group, the ANOVA was not significant $(p=0.43)$, indicating that there was no change in CBF in this group during the time interval evaluated. However, the repeated measures ANOVA for the surgical hypoperfusion group was significant $[F(6,34)=10.26, p<0.005]$. To determine critical time points at which CBF was altered in this group, and to control for multiple posthoc two-group comparisons because we were primarily interested in change in CBF relative to the pre-surgery time point, we applied the Dunnett's multiple comparisons approach to assess change in CBF across time. We found no CBF differences at day $1(p=0.07)$, day $3(p=0.99)$, or day $7(p=0.47)$ from the pre-surgery time point in the hypoperfusion group. However, CBF in the hypoperfusion group was significantly decreased from the pre-surgery time point by $28 \%$ on day $13(p=0.04), 43 \%$ on day $27(p=0.04)$ and $40 \%$ on day $34(p=0.02)$. The gradual reduction in $\mathrm{CBF}$ was further supported by a significant drop in $\mathrm{CBF}$ in the hypoperfusion mice as compared to sham mice at day $13(p=0.0001)$, day $27(p=0.02)$, and day $34(p=$ 0.0008). There were no differences in CBF between these groups on days 1,3 and 7 postsurgery. 


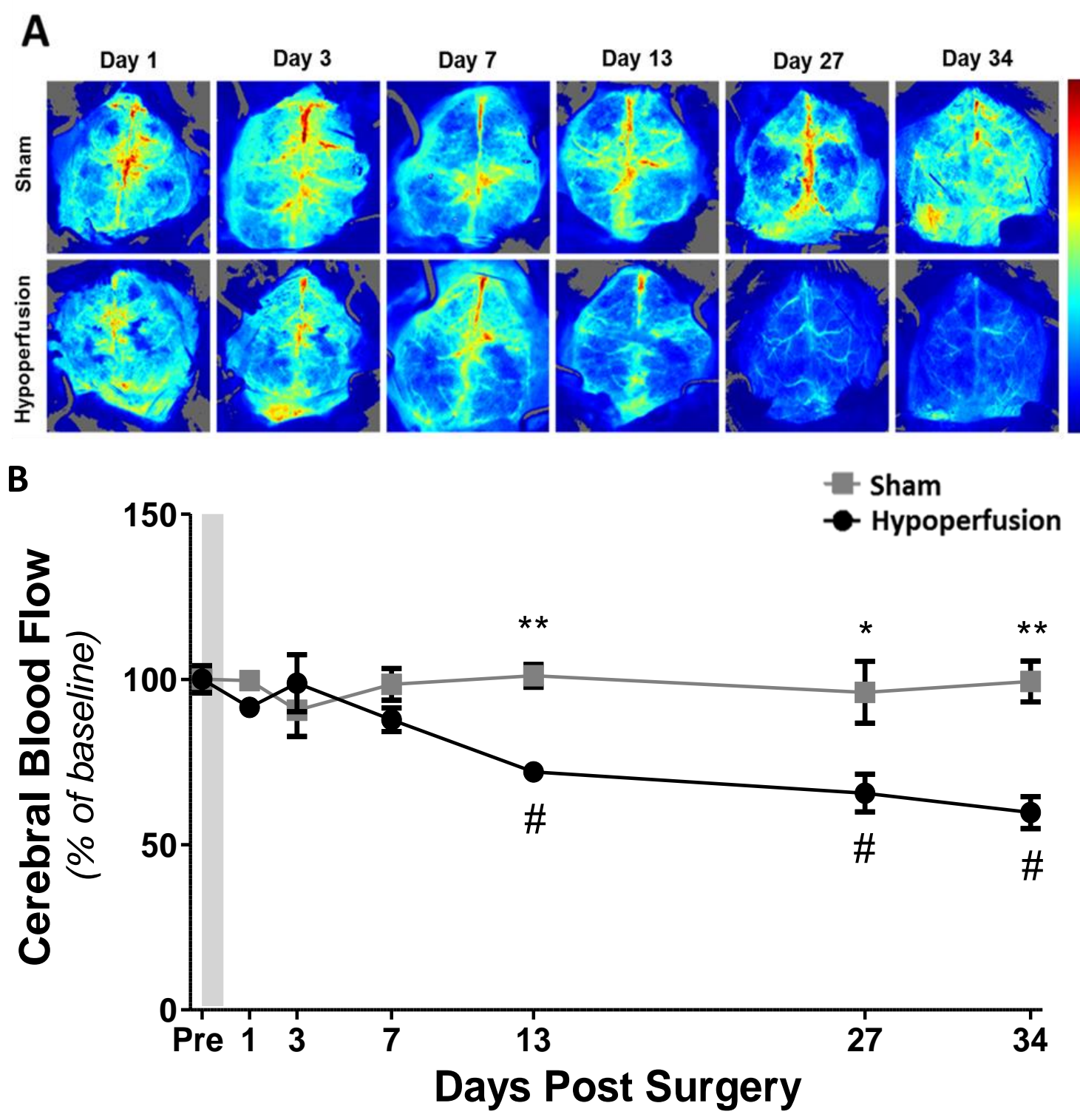

Figure 3.1. Cerebral blood flow following induction of surgical hypoperfusion. (A) Laser speckled flowmetry flux images depicting CBF over time. Red color indicates areas of high CBF while blue color indicates areas of low CBF. (B) Temporal changes in CBF measured by the laser Doppler system. Points represent means \pm SEM, sham $n=6$, hypoperfusion $\mathrm{n}=5$. $\#=p<0.05$ for the hypoperfusion group at each time point versus pre-surgical time point; $*=p<0.05 ; * *=p<0.001$ between treatment groups at each time point. 


\section{Histological Assessment of Cerebral Injury}

H\&E staining of cerebral tissue from hypoperfused animals revealed a variety of hallmark pathologies of gray and white matter (Figure 3.2). Thus, there was striking neuronal loss in the stratum pyramidale of the Cornu Ammonis 1 (CA1) and Cornu Ammonis 3 (CA3) region of the hippocampus, and loss of neurons in the stratum granulosum in the dentate gyrus (DG). We noticed hemisphere asymmetry in hippocampal and cortical injury in hypoperfused mice. Hypoperfusion is known to induce lesions that are formed by localized intense vacuolization (Wells and Wells, 1989). We observed lesions of intense vacuolization in the stratum oriens, stratum pyramidale, and stratum radiatum in the CA1 and CA3 regions of the hippocampus. There were overt degenerative lesions in the fimbria of the fornix (FF), external segment of the globus pallidus (GPe), and internal segment of the globus pallidus (GPi). These lesions contained an elevated number of nuclei, suggesting infiltration of inflammatory cells. No pathology was evident in the sham group.

Silver staining revealed severe axonal injury, identified as punctate staining observed in the stratum oriens and stratum radiatum of the CA1 region of the hippocampus of hypoperfused mice (Figure 3.3). A similar observation was made in the CA2 region. Axonal injury in both the stratum oriens and stratum radiatum of the CA3 region of the hippocampus appeared to be less affected.

Silver staining also revealed prominent injury of the corpus callosum in hypoperfused mice (Figure 3.4). Severe atrophy of the body of the corpus callosum was apparent in the hypoperfused mice. Intense silver staining of axons projecting through the corpus callosum indicated axonal damage. The appearance of gaps between axons in the corpus callosum indicated their degeneration. Additionally, axonal disorganization was a common observation 

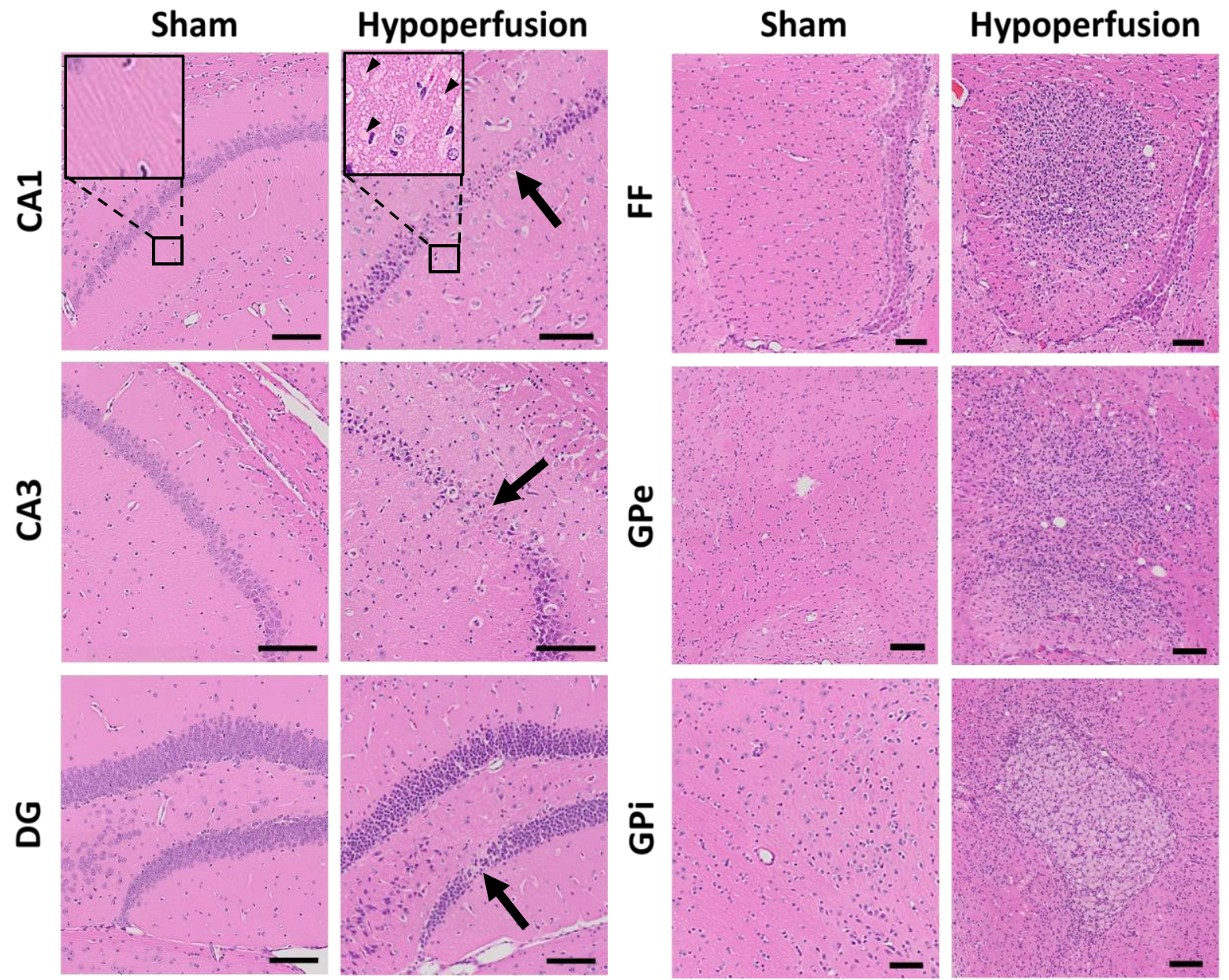

Figure 3.2. Cerebrovascular hypoperfusion causes brain tissue injury. Brightfield micrographs of H\&E stained coronal tissue sections from sham and hypoperfused group mice. Cornu ammonis 1 (CA1); cornu ammonis 3 (CA3); dentate gyrus (DG); fimbria fornix (FF); globus pallidus externus (GPe); globus pallidus internus (GPi). Arrows indicate regions of neuronal degeneration. In the magnified panel insert, arrowheads indicate vacuolization. Scale bars indicate $100 \mu \mathrm{m}$. 
in regions with extensive axonal injury. No axonal injury was apparent either in the hippocampus or in the corpus callosum of the sham mice.

\section{Discussion}

Numerous studies of cerebral pathophysiology of hypoperfusion in animal models have led to the identification of hundreds of potential therapeutic compounds. However, in clinical trials, all of these compounds have proven ineffective, or less effective than the already clinically available interventions. The lack of adequate experimental models of cerebrovascular hypoperfusion with pathophysiology analogous to humans is likely the primary cause of the loss of translation from laboratory to clinical practice. A major limitation of the current animal models is variable severity of ischemic damage that weakens the statistical power of experimental research making the identification of effective therapeutics difficult.

There are several paradigms to induce cerebrovascular hypoperfusion in mice. Essentially all of the current paradigms involve the occlusion of two (Bottiger et al., 1998; Bottiger et al., 1999), three (Carmichael, 2005; Thal et al., 2010; Onken et al., 2012) or four (Pulsinelli and Buchan, 1988; Traystman, 2003) vessels. These paradigms include, bilateral common carotid artery stenosis (Shibata et al., 2007; Matin et al., 2016; Patel et al., 2017), permanent carotid artery ligation (Ohta et al., 1997) and sequential common carotid artery occlusion (Cechetti et al., 2010). Limitations of other procedures used to induce hypoperfusion in mice include a high mortality rate (Longa et al., 1989; Connolly et al., 1996; Kitagawa et al., 1998) and inconsistency in the severity and localization of cerebral damage (Connolly et 


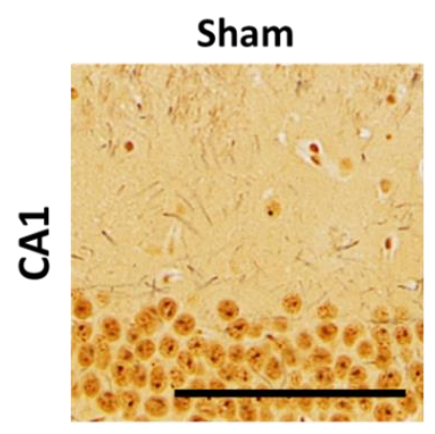

\section{Hypoperfusion}
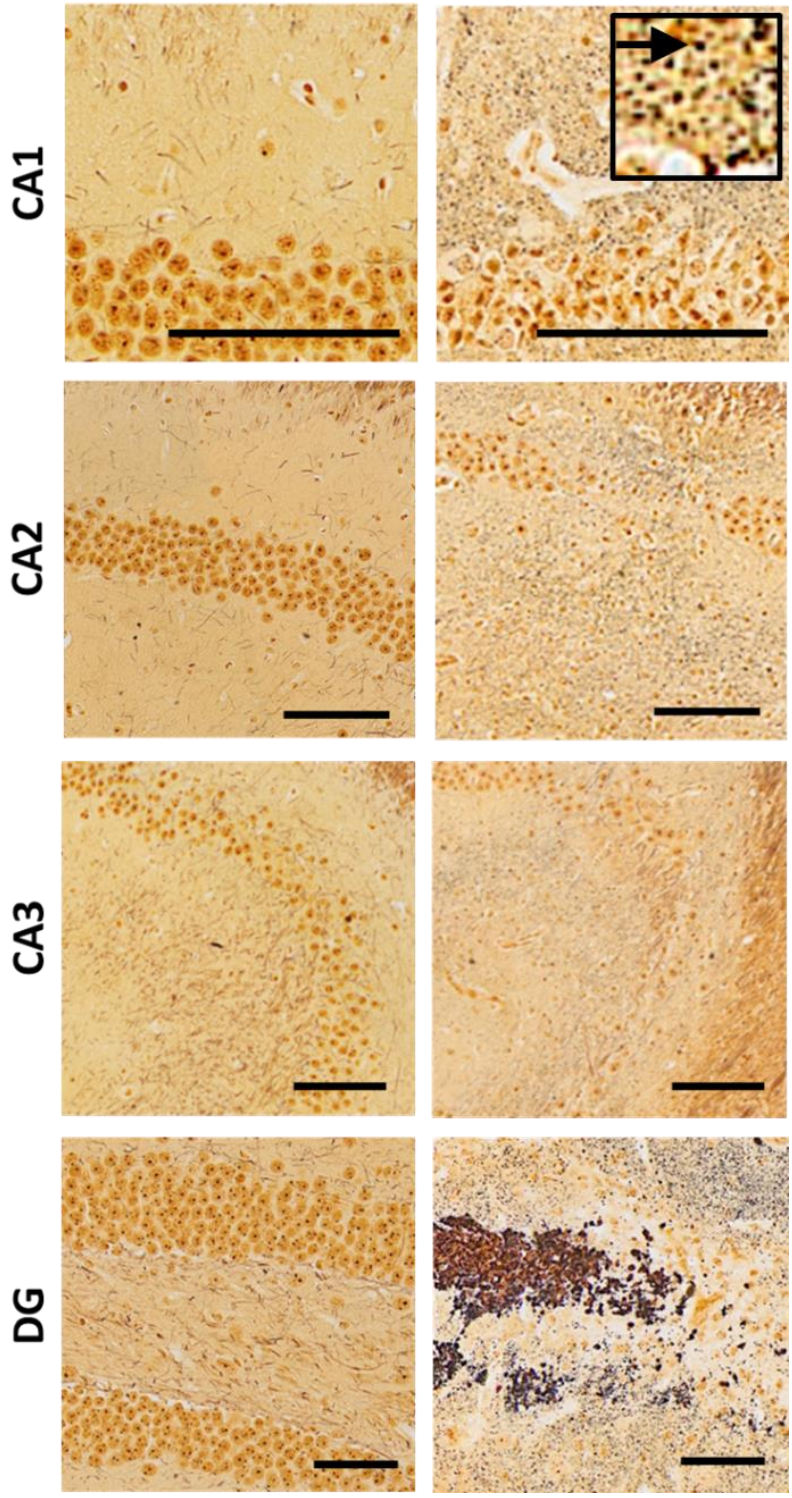

Figure 3.3. Cerebrovascular hypoperfusion causes hippocampal injury. Sliver stained tissue depicting histological changes in sham and hypoperfusion group mice. Cornu ammonis 1 (CA1); cornu ammonis 2 (CA2); cornu ammonis 3 (CA3); dentate gyrus (DG). The magnified panel insert shows degenerated axons and their punctated appearance (black arrow). Scale bars indicate $100 \mu \mathrm{m}$. 
al., 1996; Clark et al., 1997; Ohta et al., 1997; Takano et al., 1997; Matin et al., 2016; Patel et al., 2017). Using the present paradigm, we were able to induce a consistent time-dependent reduction of cerebral blood flow that resulted in limited mortality and relatively conserved damage severity and localization.

Models of cerebrovascular hypoperfusion that use bilateral CCA stenosis with microcoils have demonstrated pathology to white matter without gray matter damage after 30 days of chronic reduction of CBF (Shibata et al., 2004). However, in studies where microcoils are placed on both common carotid arteries in mice have demonstrated a maximum reduction of $\mathrm{CBF}$ to a substantial $51 \%$ of baseline within 2 hours followed by a progressive recovery of CBF that reached $82 \%$ by day 30 (Shibata et al., 2004). Other models of hypoperfusion that utilize stenosis of CCA have demonstrated an acute reduction of $\mathrm{CBF}$ to $~ 55 \%$ on the first day (Kitamura, et al., 2016; Srinivasan et al., 2015; Hattori et al., 2016). Growth of collateral vessels occurs only after a few days of hypoperfusion resulting in a recovery of CBF over time (Srinivasan et al., 2015). This suggests that the greatest damage occurs during the first days after surgery, and once CBF is restored, the tissue recovers. This makes it difficult to gauge accurately the extent of damage caused by permanently reduced blood flow as in cerebral hypoperfusion in humans. In our model, the stenosis of the CCA develops gradually over time. The irreversibility of CBF reduction prevents the recovery of cerebral tissue, which may explain the injury of both the white and gray matter.

In a study on hypertensive rats using a two-vessel gradual occlusion with ameroid constrictor rings on both common carotid arteries reduced CBF to $78 \%$ of baseline within 3 hours post-surgery and after one day reached a maximum reduction of $70 \%$ that persisted to day seven, before recovering to 80 and $82 \%$ by day 14 and 28, respectively (Kitamura et al., 
Sham
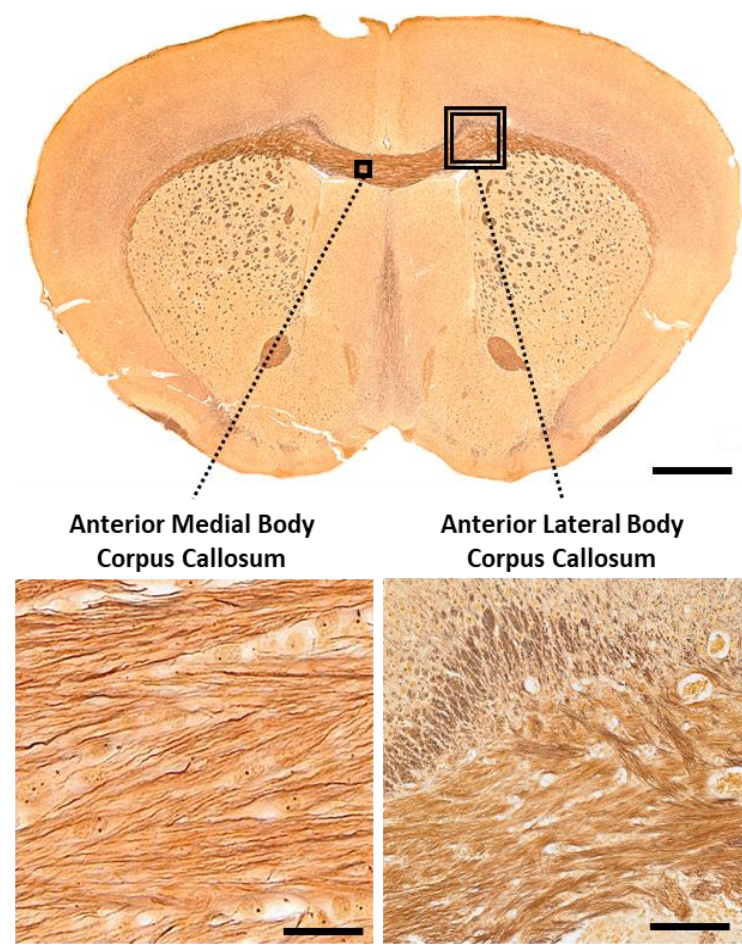

Hypoperfusion
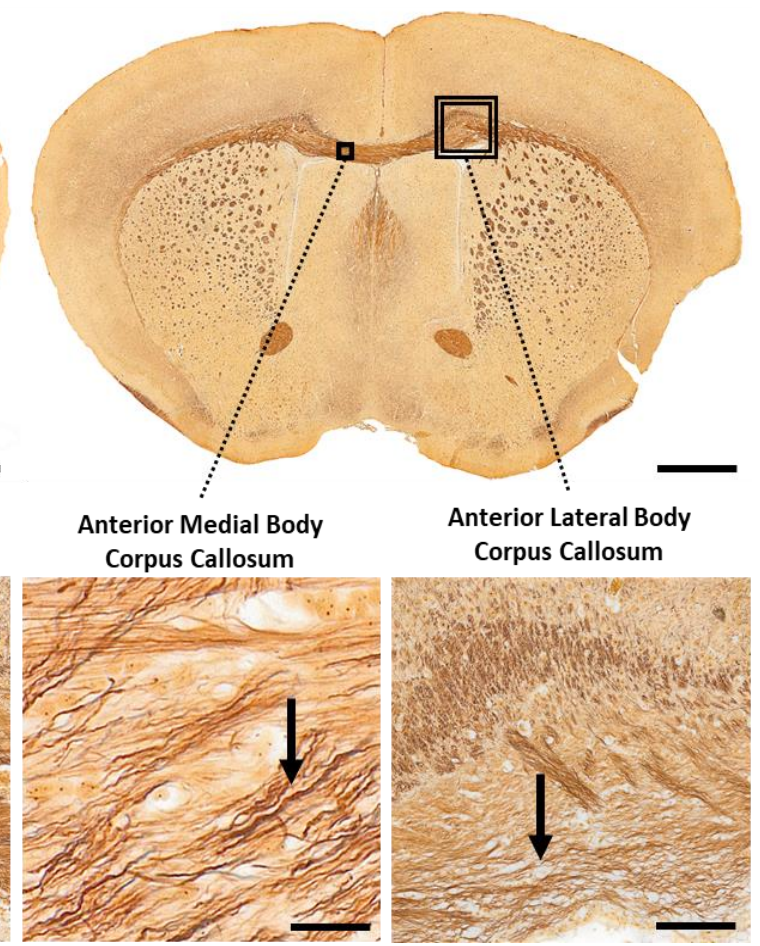

Corpus Callosum

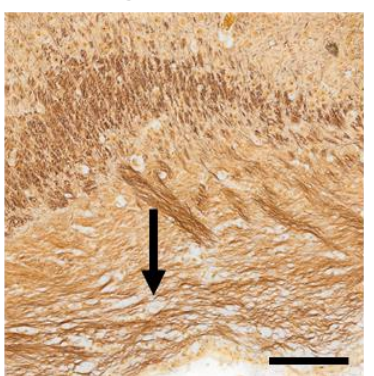

Figure 3.4. Cerebrovascular hypoperfusion causes corpus callosum injury. Silver stained whole coronal tissue sections of the brain and magnified regions of the medial and lateral corpus callosum in sham and hypoperfused mice. Black arrow in the medial and lateral corpus callosum indicate axonal degeneration (threadlike) and disorganization (intense and non-bundled), respectively. Scale bars indicate $1 \mathrm{~mm}$ (whole coronal sections) and $20 \mu . m$ (magnified panels). 
2016). In contrast, we found no CBF differences at day 1, 3, and 7 from the pre-surgery time point in the hypoperfusion group. However, $\mathrm{CBF}$ in the hypoperfusion group was significantly reduced to $72 \%$ on day 13 and $57 \%$ by day 27 . Additionally, we observed little to no recovery of $\mathrm{CBF}$ over the 34 days of hypoperfusion, which was maintained at $60 \%$ on the final day of the experiment. In agreement with the findings of Kitamura et al. (2016) that found lesion in the corpus callosum after 28 days of hypoperfusion, our data demonstrate similar injury to the corpus callosum. In addition, we observed severe damage to the hippocampus, cortex and subcortical regions, albeit asymmetric to the hemispheres. Noteworthy, these differences in findings compared to our study using normotensive mice may be attributed to the characteristic hemodynamic properties of hypertensive rats.

In conclusion, we have established a novel murine model of irreversible hypoperfusion that develops gradually and progressively. The model results in a substantial reduction of CBF and produces consistent localization and severity of cerebral injury in the CA1, CA3, DG, FF, $\mathrm{GPe}, \mathrm{GPi}$, and corpus callosum with a relatively low mortality rate. The model provides a tool for investigating cerebral vascular disease with chronic cerebral hypoperfusion, which is implicated in the development of white matter disease and lacunar infarcts. 


\section{Acknowledgement}

The authors thank Dr. Gregory Konat for revising and editing the manuscript. This study is supported by the Helen Marie Lewis Medical Research Foundation (to JWS, XR, and AM), NIH CoBRE (P20 GM109098 to JWS), AHA SDG (16SDG31170008 to XR), NIH T32 (AG052375 to JWS), and funding from the Department of Neurology at WVU (to AM). 


\section{References}

Akiguchi I, Tomimoto H, Wakita H, Kawamoto Y, Matsuo A, Ohnishi K, Watanabe T, Budka H (2004) Topographical and cytopathological lesion analysis of the white matter in Binswanger's disease brains. Acta neuropathologica 107:563-570.

Black S, Gao F, Bilbao J (2009) Understanding white matter disease: imaging-pathological correlations in vascular cognitive impairment. Stroke; a journal of cerebral circulation 40:S48-52.

Bottiger BW, Schmitz B, Wiessner C, Vogel P, Hossmann KA (1998) Neuronal stress response and neuronal cell damage after cardiocirculatory arrest in rats. Journal of cerebral blood flow and metabolism : official journal of the International Society of Cerebral Blood Flow and Metabolism 18:1077-1087.

Bottiger BW, Teschendorf P, Krumnikl JJ, Vogel P, Galmbacher R, Schmitz B, Motsch J, Martin E, Gass P (1999) Global cerebral ischemia due to cardiocirculatory arrest in mice causes neuronal degeneration and early induction of transcription factor genes in the hippocampus. Brain research Molecular brain research 65:135-142.

Carmichael ST (2005) Rodent models of focal stroke: size, mechanism, and purpose. NeuroRx : the journal of the American Society for Experimental NeuroTherapeutics 2:396-409.

Cechetti F, Worm PV, Pereira LO, Siqueira IR, C AN (2010) The modified 2VO ischemia protocol causes cognitive impairment similar to that induced by the standard method, but with a better survival rate. Brazilian journal of medical and biological research $=$ Revista brasileira de pesquisas medicas e biologicas 43:1178-1183.

Clark WM, Lessov NS, Dixon MP, Eckenstein F (1997) Monofilament intraluminal middle cerebral artery occlusion in the mouse. Neurological research 19:641-648.

Connolly ES, Jr., Winfree CJ, Stern DM, Solomon RA, Pinsky DJ (1996) Procedural and strain-related variables significantly affect outcome in a murine model of focal cerebral ischemia. Neurosurgery 38:523-531; discussion 532. 
Hattori Y, Enmi J, Iguchi S, Saito S, Yamamoto Y, Nagatsuka K, Iida H, Ihara M (2016) Substantial Reduction of Parenchymal Cerebral Blood Flow in Mice with Bilateral Common Carotid Artery Stenosis. Scientific reports 6:32179.

Hattori Y, Enmi J, Kitamura A, Yamamoto Y, Saito S, Takahashi Y, Iguchi S, Tsuji M, Yamahara K, Nagatsuka K, lida H, Ihara M (2015) A novel mouse model of subcortical infarcts with dementia. The Journal of neuroscience : the official journal of the Society for Neuroscience 35:3915-3928.

Kitagawa K, Matsumoto M, Yang G, Mabuchi T, Yagita Y, Hori M, Yanagihara T (1998) Cerebral ischemia after bilateral carotid artery occlusion and intraluminal suture occlusion in mice: evaluation of the patency of the posterior communicating artery. Journal of cerebral blood flow and metabolism : official journal of the International Society of Cerebral Blood Flow and Metabolism 18:570-579.

Kitamura A, Saito S, Maki T, Oishi N, Ayaki T, Hattori Y, Yamamoto Y, Urushitani M, Kalaria RN, Fukuyama H, Horsburgh K, Takahashi R, Ihara M (2016) Gradual cerebral hypoperfusion in spontaneously hypertensive rats induces slowly evolving white matter abnormalities and impairs working memory. Journal of cerebral blood flow and metabolism : official journal of the International Society of Cerebral Blood Flow and Metabolism 36:1592-1602.

Kitamura A, Manso Y, Duncombe J, Searcy J, Koudelka J, Binnie M, Webster S, Lennen R, Jansen M, Marshall I, Ihara M, Kalaria RN, Horsburgh K (2017) Long-term cilostazol treatment reduces gliovascular damage and memory impairment in a mouse model of chronic cerebral hypoperfusion. Scientific reports 7:4299.

Kynast J, Lampe L, Luck T, Frisch S, Arelin K, Hoffmann KT, Loeffler M, Riedel-Heller SG, Villringer A, Schroeter ML (2017) White matter hyperintensities associated with small vessel disease impair social cognition beside attention and memory. Journal of cerebral blood flow and metabolism : official journal of the International Society of Cerebral Blood Flow and Metabolism:271678X17719380. 
Longa EZ, Weinstein PR, Carlson S, Cummins R (1989) Reversible middle cerebral artery occlusion without craniectomy in rats. Stroke; a journal of cerebral circulation 20:8491.

Matin N, Fisher C, Jackson WF, Dorrance AM (2016) Bilateral common carotid artery stenosis in normotensive rats impairs endothelium-dependent dilation of parenchymal arterioles. American journal of physiology Heart and circulatory physiology 310:H1321-1329.

Ohta H, Nishikawa H, Kimura H, Anayama H, Miyamoto M (1997) Chronic cerebral hypoperfusion by permanent internal carotid ligation produces learning impairment without brain damage in rats. Neuroscience 79:1039-1050.

Onken M, Berger S, Kristian T (2012) Simple model of forebrain ischemia in mouse. Journal of neuroscience methods 204:254-261.

Pantoni L, Garcia JH (1997) Pathogenesis of leukoaraiosis: a review. Stroke; a journal of cerebral circulation 28:652-659.

Patel A, Moalem A, Cheng H, Babadjouni RM, Patel K, Hodis DM, Chandegara D, Cen S, He S, Liu Q, Mack WJ (2017) Chronic cerebral hypoperfusion induced by bilateral carotid artery stenosis causes selective recognition impairment in adult mice. Neurological research 39:910-917.

Pulsinelli WA, Buchan AM (1988) The four-vessel occlusion rat model: method for complete occlusion of vertebral arteries and control of collateral circulation. Stroke; a journal of cerebral circulation 19:913-914.

Shibata M, Ohtani R, Ihara M, Tomimoto H (2004) White matter lesions and glial activation in a novel mouse model of chronic cerebral hypoperfusion. Stroke; a journal of cerebral circulation 35:2598-2603.

Shibata M, Yamasaki N, Miyakawa T, Kalaria RN, Fujita Y, Ohtani R, Ihara M, Takahashi R, Tomimoto H (2007) Selective impairment of working memory in a mouse model of chronic cerebral hypoperfusion. Stroke; a journal of cerebral circulation 38:2826-2832. 
Srinivasan VJ, Yu E, Radhakrishnan H, Can A, Climov M, Leahy C, Ayata C, EikermannHaerter K (2015) Micro-heterogeneity of flow in a mouse model of chronic cerebral hypoperfusion revealed by longitudinal Doppler optical coherence tomography and angiography. Journal of cerebral blood flow and metabolism : official journal of the International Society of Cerebral Blood Flow and Metabolism 35:1552-1560.

Takano K, Tatlisumak T, Bergmann AG, Gibson DG, 3rd, Fisher M (1997) Reproducibility and reliability of middle cerebral artery occlusion using a silicone-coated suture (Koizumi) in rats. Journal of the neurological sciences 153:8-11.

Thal SC, Thal SE, Plesnila N (2010) Characterization of a 3-vessel occlusion model for the induction of complete global cerebral ischemia in mice. Journal of neuroscience methods 192:219-227.

Traystman RJ (2003) Animal models of focal and global cerebral ischemia. ILAR journal 44:85-95.

Uchihara T (2007) Silver diagnosis in neuropathology: principles, practice and revised interpretation. Acta neuropathologica 113:483-499.

Vasquez BP, Zakzanis KK (2015) The neuropsychological profile of vascular cognitive impairment not demented: a meta-analysis. Journal of neuropsychology 9:109-136.

Wakita H, Tomimoto H, Akiguchi I, Kimura J (1995) Protective effect of cyclosporin A on white matter changes in the rat brain after chronic cerebral hypoperfusion. Stroke; a journal of cerebral circulation 26:1415-1422.

Wells GA, Wells M (1989) Neuropil vacuolation in brain: a reproducible histological processing artefact. Journal of comparative pathology 101:355-362.

Xiong Y, Mahmood A, Lu D, Qu C, Kazmi H, Goussev A, Zhang ZG, Noguchi CT, Schallert T, Chopp M (2008) Histological and functional outcomes after traumatic brain injury in mice null for the erythropoietin receptor in the central nervous system. Brain research 1230:247-257. 


\section{CHAPTER 4}

\section{Amyloid- $\beta$ Causes Mitochondrial Dysfunction via a $\mathrm{Ca}^{2+}$-Driven Upregulation of Oxidative Phosphorylation and Superoxide Production in Cerebrovascular Endothelial Cells}

Dominic D. Quintana ${ }^{1}$, Jorge A. Garcia ${ }^{2}$, Yamini Anantula ${ }^{2}$, Stephanie L. Rellick ${ }^{1}$, Elizabeth B. Engler-Chiurazzi ${ }^{2}$, Saumyendra N. Sarkar ${ }^{1}$, Candice M. Brown ${ }^{2}$ and James W. Simpkins ${ }^{1}$

${ }^{1}$ Department of Physiology and Pharmacology

${ }^{2}$ Department of Neuroscience

Center of Basic and Translational Stroke Research

West Virginia University, Morgantown, West Virginia, 26506

\section{Author Contribution Statement}

DDQ designed studies, conducted studies and composed the manuscript. JAG, YA, SR, EBEC, SNS, and CMB aided with studies, analyzed data, and revised the manuscript. JWS designed studies and revised the manuscript. 


\section{Abstract}

Cerebrovascular pathology is pervasive in Alzheimer's disease (AD), yet it is unknown whether cerebrovascular dysfunction contributes to the progression or etiology of AD. In human subjects and in animal models of $\mathrm{AD}$, cerebral hypoperfusion and hypometabolism are reported to manifest during the early stages of the disease and persist for its duration. Amyloid$\beta(\mathrm{A} \beta)$ is known to cause cellular injury in both neurons and endothelial cells by inducing the production of reactive oxygen species (ROS) and disrupting intracellular $\mathrm{Ca}^{2+}$ homeostasis. We present a mechanism for mitochondrial degeneration caused by the production of mitochondrial superoxide, which is driven by increased mitochondrial $\mathrm{Ca}^{2+}$ uptake. We found that persistent superoxide production injures mitochondria and disrupts electron transport in cerebrovascular endothelial cells. These observations provide a mechanism for the mitochondrial deficits that contribute to cerebrovascular dysfunction in patients with $\mathrm{AD}$. 
Keywords: Amyloid- $\beta$, Vascular Endothelial Cells, Mitochondria, Calcium, Superoxide 


\section{Introduction}

Alzheimer's disease (AD) is the most common form of senile dementia and is characterized by progressive neurodegeneration and cognitive decline. Parenchymal plaques are a hallmark of $\mathrm{AD}$ and are formed by the deposition of amyloid- $\beta$ polymers (Querfurth and LaFerla 2010; Castellani et al. 2010). The contribution of amyloid- $\beta$ deposition to the manifestation and progression of $\mathrm{AD}$ has been the focus of investigation since the discovery of the peptide. Remarkably, $90 \%$ of patients with AD demonstrate cerebral amyloid angiopathy (CAA), a neuropathological disease characterized by the deposition of amyloid- $\beta$ on the walls of cerebral vasculature (Janson 2015; DeSimone et al. 2017; Vinters 1987; Han et al. 2015).

Cerebrovascular comorbidities are common in $\mathrm{AD}$, as many as $92 \%$ of $\mathrm{AD}$ patients demonstrate ischemic white matter lesions that resemble arteriosclerosis of small vessels. Microvascular degeneration can have obvious detrimental consequences to cerebral tissue. A number of reports show regional hypoperfusion, hypometabolism, and blood-brain barrier hyperpermeability in subjects with AD (de la Torre 2004; Brundel et al. 2012a; Brundel et al. 2012b). To date, a large prevalence of patients with $\mathrm{AD}$ are at risk for developing severe vascular conditions that include: hemorrhagic stroke, spontaneous cerebral emboli, cerebral microinfarctions, and microhemorrhages (Brundel et al. 2012a; Chi et al. 2013; Purandare and Burns 2009; Tolppanen et al. 2013). A growing body of evidence has emerged documenting cerebrovascular dysfunction preceding cognitive decline in AD patients, (de la Torre 2010; Jellinger 2010; Kalaria 2010) suggesting that vascular dysfunction may play a causative role in the emergence of AD. However, the mechanism(s) by which amyloid- $\beta$ exerts it cytotoxic effects on the cerebrovasculature are not yet known. 
It is known that bioenergetic deficits occur in neurons exposed to $A \beta$ and that mitochondrial dysfunction precedes the onset of pathology in $\mathrm{AD}$ (Yao et al. 2009; Behl et al. 1994). These observations suggest that mitochondrial dysfunction during the early stages of $\mathrm{AD}$ (non-symptomatic) compromise cerebrovascular function resulting in cerebral $\mathrm{A} \beta$ retention, vascular degeneration, and hypoperfusion, which together may initiate the cognitive decline and symptomatic phase of $\mathrm{AD}$. Observations of postmortem brain tissue from $\mathrm{AD}$ patients have revealed extensive mitochondrial network disruption, presenting as evidence of excessive mitochondrial fission and fragmented cristae (Trimmer et al. 2000; Hirai et al. 2001; Manczak et al. 2011; Wang et al. 2017). In cultured cortical neurons, $A \beta$ exposure results in deficits of synaptic mitochondria and reduced mitochondrial transport, likely contributing to the synaptic dysfunction in $\mathrm{AD}$ (Du et al. 2010). Together, these observational studies indicate a pivotal role for mitochondrial injury in the cytotoxicity of $A \beta$. Cellular production of reactive oxygen species (ROS) has been shown to result in S-nitrosylation of Drp-1 resulting in aberrant mitochondrial fission, synaptic loss, and neuronal damage (Cho et al. 2009). Increased production of $\mathrm{A} \beta$-induced ROS has also been demonstrated in cerebrovascular endothelial cells and causes cell dysfunction, BBB disruption, and degeneration (Han et al. 2015; Park et al. 2004). The mechanism by which $A \beta$ induces the production of ROS in endothelial cells is thought to involve the activation of the NADPH oxidase membrane-bound subunit, Nox (Drummond et al. 2011; Miller et al. 2005). Genetic ablation of vascular endothelial cell Nox2 or inhibiting NADPH assembly abrogates ROS and vascular dysfunction induced by A $\beta$ (Park et al. 2004; Park et al. 2007; Park et al. 2005).

In the present study, we describe a mechanism for $A \beta$-induced cerebrovascular endothelial cell injury. We provide experimental evidence from primary cultures of vascular 
endothelial cells and a bEnd. 3 cell line exposed to $A \beta$, which showed increased production of mitochondrial superoxide via direct $A \beta_{1-42}$ interaction with mitochondria. $A \beta$ induced a dosedependent increase in mitochondrial superoxide production that paralleled an increase in mitochondrial oxygen consumption and ATP production, suggesting mitochondrial stress. We found that $A \beta$ exerts these effects by increasing mitochondrial $\mathrm{Ca}^{2+}$ concentration, driving hyper-oxidative metabolism and increasing ROS production by mitochondria. Furthermore,

removal of extracellular $\mathrm{Ca}^{2+}$ abrogates the $\mathrm{A} \beta_{1-42}$-induced increase of mitochondrial oxygen consumption, ATP production, $\mathrm{Ca}^{2+}$ accumulation, superoxide production and cerebrovascular endothelial cell death. These $\mathrm{A} \beta$-induced effects on mitochondrial function may provide a mechanism for the mitochondrial dysfunction and deficits observed in AD and provide evidence for a therapeutic intervention that targets extracellular calcium.

\section{Materials and Methods}

\section{Study Design}

All experiments were performed using either primary cerebrovascular endothelial cells from mice or the bEnd.3 [BEND3] (RRID: CVCL_0170, ATCC@ CRL-2299) mouse brain capillary endothelial cell line. The brain endothelial cell line is not listed as a commonly misidentified cell line by the International Cell Line Authentication Committee. Initial authentication of the bEnd. 3 cell line was performed by the vendor; no further authentication was performed in the laboratory. Cells were cultured with Dulbecco's modified Eagles medium (DMEM) (Cat. No. SH30022.01 (2019), HyClone GE) supplemented with 10\% fetal bovine serum (Cat. No. S12450 (2019), Atlanta Biosciences) and 1\% penicillin streptomycin (Cat. No. SV30010 (2019), HyClone GE) (DMEM+). Cells were plated in either 96-well assay 
plates $\left(1.5 \times 10^{4}\right.$ cells/well $), 24-w e l l$ culture plates $\left(3.5 \times 10^{4}\right.$ cells/well $)$, or $10-\mathrm{cm}$ culture dishes $\left(1.5 \times 10^{5}\right.$ cells/dish $)$ and incubated at $37^{\circ} \mathrm{C}$ in a humidified incubator under $5 \% \mathrm{CO}_{2}$. Primary cerebrovascular endothelial cells were subcultivated every 4 days (ratio $=1: 2$ ) and bEnd.3 cells every 3 days (ratio $=1: 8$ ). The first and second passage of primary cell cultures were retained for cryopreservation. For experimental purposes, primary endothelial cell subcultures were utilized beginning at passage 3 up until a maximum passage number of 6 . For experiments that used the bEnd. 3 cell line were performed on cells at passage $15-20$. This study was not preregistered or blinded. Stratified randomization was employed in all experiments that utilized cultured cells to prevent any experimental condition to be statically positioned across all experiments.

\section{Animal Usage}

Three-month old C57BL6 (National Institutes on Aging) pregnant female mice $(n=4)$ were used to produce primary cerebrovascular endothelial cell cultures for the purposes of this study. Primary cell cultures were prepared from embryos at gestational day 19 from each of the pregnant mice. Primary cells cultures were prepared from each mouse over four separate occasion, around 12:00pm eastern time. Pregnant mice produced an average of 6 embryos that were harvested and pooled together to produce a culture of primary cerebrovascular endothelial cells. Therefore, each mouse produced a single culture of primary cells and each of the four instances of prepared primary cultures was used to replicate the experiments described in the study. Power analysis for ANOVA designs indicated a sample size of 3 pregnant mice (power $=0.999$ ) for an effect size of $\Delta=1.25$. Mice were housed in accordance to IACUC guidelines of West Virginia University (protocol \#: 13-0704). Animals were maintained under a light / dark cycle (12: 12) with food and water available ad libitum. For all procedures performed in 
this study that involved the use of laboratory animals were carried out in accordance with the National Institutes of Health guide for the care and use of laboratory animals (NIH Publication No. 8023, revised 1978) and in compliance with the ARRIVE guidelines.

\section{Preparation of Primary Cerebrovascular Endothelial Cell Cultures}

Primary cerebrovascular endothelial cells were prepared from embryonic mice. At embryonic day 19, pregnant mice were deeply anesthetized with $4 \%$ isoflurane diffused into $70 \%$ nitrogen and $30 \%$ oxygen mixture. After confirming deep anesthetization via tail pinch, mice were euthanized by cervical dislocation. Mice received a vertical incision to the abdomen. Through the incision, each fetus was extracted, removed from the amniotic sack, and decapitated. The collected fetal heads were immediately immersed in ice-cold HBSS buffer (Cat. No. 14025-092 (2019), Gibco). Each brain was extracted through an incision made along the superior sagittal suture followed by the removal of the cerebellum for discard. The remaining tissue was placed into a $50 \mathrm{~mL}$ centrifuge tube containing ice-cold DMEM. The brain tissue from each fetus was consolidated into a single $50 \mathrm{~mL}$ centrifuge tube containing $20 \mathrm{~mL}$ of ice-cold DMEM then centrifuged at 2500 times gravity for $3 \mathrm{~min}$. The supernatant was decanted and the tissue pellet was resuspended in $1 \mathrm{~mL}$ of ice-cold DMEM. The suspended tissue was then transferred to a Dounce homogenizer that was kept on ice. After homogenization, the tissue was transferred into a $50 \mathrm{~mL}$ tube containing $1.5 \%$ HEPES $1 \mathrm{M}$ (Cat. No. H0887-100ML (2019), Sigma Aldrich) in $20 \mathrm{~mL}$ HBSS. The tissue was briefly vortexed followed by centrifugation at 2000 times gravity for $10 \mathrm{~min}$ at $4{ }^{\circ} \mathrm{C}$. Supernatant was decanted and the pellet was resuspended in a solution of $18 \%$ (w/v) dextran (Cat. No. 3139250G (2018), Sigma Aldrich) and 1.5\% HEPES 1M in $20 \mathrm{~mL}$ HBSS. The tube was mixed by inverting then centrifuged at 5000 times gravity for $10 \mathrm{~min}$ at $4{ }^{\circ} \mathrm{C}$. Once centrifuged, the 
supernatant along with the cholesterol interphase was discarded; the pellet was then resuspended in $20 \mathrm{~mL}$ of warm DMEM containing $10 \%$ FBS and $1 \%$ penicillin streptomycin. Vessels were isolated from the suspension by passing the solution through a $50 \mu \mathrm{m}$ filter, and rinsed with $3 \mathrm{~mL}$ of DMEM. Isolated vessels were removed from the filter by immersion in warm DMEM containing 10\% FBS and 1\% penicillin streptomycin. Collected vessels were centrifuged in a $50 \mathrm{~mL}$ tube at 2000 times gravity for $3 \mathrm{~min}$, resuspended in $1 \mathrm{~mL}$ of DMEM and plated in $10 \mathrm{~cm}$ culture dishes. Vessels were allowed to reach $65 \%$ confluence before passaging and several passages were performed before experimental use. This procedure yields cerebrovascular endothelial cell cultures primarily of capillary in origin.

\section{Mitochondria Isolation from Cerebrovascular Endothelial Cells}

Mitochondrial isolation was performed on primary cerebrovascular endothelial cells via a previously reported technique (Lampl et al., 2015). Briefly, cells were cultured to $85 \%$ confluence then harvested with a cell scraper and collected into a $50 \mathrm{~mL}$ centrifuge tube. Endothelial cells were pelleted by centrifugation at 2000 times gravity for $3 \mathrm{~min}$, supernatant was discarded and pellet resuspended in $8 \mathrm{~mL}$. With a Dounce homogenizer, cell membranes were fractured, releasing the mitochondria into the medium. The homogenate was transferred into a $15 \mathrm{~mL}$ centrifuge tube then aspirated several times using a $10 \mathrm{~mL}$ syringe with a 27 gauge needle. The homogenate was centrifuged at 800 times gravity to pellet the cell debris. After centrifugation, the supernatant was decanted into a new $15 \mathrm{~mL}$ tube then distributed into $2 \mathrm{~mL}$ microcentrifuge tubes. Microcentrifuge tubes were centrifuged at 10000 times gravity for $5 \mathrm{~min}$ at $5^{\circ} \mathrm{C}$ to pellet the mitochondria. Once centrifugation completed, the supernatant was discarded and the mitochondrion pellet was resuspended in a solution of $10 \mathrm{mM}$ TrisMOPS (Cat. No. M-8899 (2019), Sigma Aldrich) at pH 7.4, 1 mM EGTA-Tris (Cat. No. 
E8145-10G (2019), Sigma Aldrich), and 200 mM sucrose (Cat. No. S5-12 (2018), Fisher Scientific).

\section{Aß $\beta_{1-42}$ Preparation}

Four separate preparations of $A \beta_{1-42}$ (Cat. No. 03112 (2019), Thermo Fisher Scientific) were performed over the duration of the study. For each preparation, $1 \mathrm{mg}$ of lyophilized $\mathrm{A} \beta_{1 \text { - }}$ 42 monomers was suspended in $167 \mu \mathrm{L}$ of HPLC grade water (Cat. No. AM9937 (2018), Ambion) and incubated at room temperature (RT) for 5 min. The dissolved $A \beta_{1-42}$ was then diluted to $230 \mu \mathrm{M}$ by adding $833 \mu \mathrm{L}$ of $\mathrm{Ca}^{2+}$-free phosphate buffered saline (PBS) (Cat. No. P4417-100TAB (2019), 0.01M, Sigma Aldrich) and incubated for $48 \mathrm{~h}$ at $37^{\circ} \mathrm{C}$ for polymerization. After polymerization, $A \beta_{1-42}$ was separated into $50 \mu \mathrm{L}$ aliquots and stored at $80^{\circ} \mathrm{C}$. The final product produced $230 \mu \mathrm{M} \mathrm{A} \beta_{1-42}$ at mixed polymerization states.

\section{Endothelial Cell Death Assay}

To assess $A \beta_{1-42}$ - induced cell death, bEnd.3 cells were seeded in 24-well plates at a density of $2.5 \times 10^{4}$ and allowed to reach $70-85 \%$ confluency. Then, vascular endothelial cells were rinsed with pre-warmed DMEM containing 10\% FBS and 1\% penicillin streptomycin. $\mathrm{A} \beta_{1-42}$ was prepared in 5-, 9-, and $18-\mu \mathrm{M}$ solutions diluted in DMEM+ then added to the wells containing the vascular endothelial cells. Cerebrovascular endothelial cells were exposed to $\mathrm{A} \beta_{1-42}$ for a duration of $24 \mathrm{~h}$. After exposure, cells were rinsed 3 times with $0.1 \mathrm{M}$ PBS, and then fixed with paraformaldehyde (PFA) 4\% (Cat. No. 15714 (2018), Electron Microscopy Sciences) for $15 \mathrm{~min}$ at room temperature (RT). After fixation, endothelial cells were washed 2 times for 5 min with 0.1M PBS containing 0.045\% Tween-20 (Cat. No. P1379-500ML (2018), Sigma Aldrich). Residual detergent was removed with an additional 2 washes, each for 5 min. Nuclei were stained with $50 \mathrm{nM}$ 4', 6-diamidino-2-phenylindole (DAPI) (Cat. No. D- 
21490 (2017), Molecular Probes) counter stain. After nuclear staining, cells were washed 3 times for 5 min with 0.1M PBS and imaged on an EVOS2 FL Auto microscope (Invitrogen). Cells were imaged at 10x magnification and fluorescence excitation using a mercury arc lamp and a DAPI filter set. Three images were acquired for each well and saved as tiff-formatted images. The number of nuclei in each of the three images were averaged and the average number of nuclei was multiplied by the area of the well floor divided by the microscope frame size, yielding the total number of cells per well. Cell death by chronic exposure to $A \beta_{1-42}$ was assessed using the live-dead probe (Cat. No. R37609 (2019), Invitrogen). Cells were plated on 96-well assay plates and allowed 24h of growth before receiving $9 \mu \mathrm{M} \mathrm{A} \beta_{1-42}$ in DMEM+ for $48,72,96,122 \mathrm{~h}$. After exposure, dead and live cells were labeled and quantified.

\section{Mitochondrial Superoxide Assay}

Mitochondrial superoxide production in response to $A \beta_{1-42}$ exposure was quantified using MitoSOX Red (Cat. No. M36008 (2019), Molecular Probes) according to the manufacturer's guidelines. Cerebrovascular endothelial cells were seeded on 96-well plates at a density of $1.5 \times 10^{4}$ cells per well. Endothelial cells were allowed to proliferate for $24 \mathrm{~h}$ ( 85\% confluency). Cells were rinsed twice with pre-warmed DMEM and then exposed to 0-, 5-, 9-, and 18- $\mu \mathrm{MA} \beta_{1-42}$ for $24 \mathrm{~h}$. After exposure to $A \beta_{1-42}$, cells were rinsed 3 times with warm DMEM+ and endothelial cells were loaded with $5 \mu \mathrm{M}$ MitoSOX Red diluted in DMEM+ for 10 min at $37^{\circ} \mathrm{C}$. After incubation, cells were rinsed 3 times with warm DMEM+. MitoSOX fluorescence was measured with a spectrophotometer by endpoint kinetic set to an excitation of $510 \mathrm{~nm}$ and an emission of $580 \mathrm{~nm}$. Each well was measured in relative fluorescence units (RFU). The RFU measurement from each well per group $(n=12)$ was normalized to the average RFU of the control ( $\left.0 \mu \mathrm{M} \mathrm{A} \beta_{1-42}\right)$ group to produce a fold-change value. 


\section{Endothelial Cell ATP Content Assay}

Endothelial cell ATP content was quantified using the CellTiter-Glo luminescent assay kit (Cat. No. G7571 (2018), Promega) according to the manufacturer's guidelines. The bEnd.3 cells were seeded on 96-well plates at a density of $1.5 \times 10^{4}$ cells per well then allowed to proliferate for $24 \mathrm{~h}$ ( $\sim 85 \%$ confluency). Endothelial cells were rinsed twice with warm DMEM+ then exposed to 5-, 9-, and $18-\mu \mathrm{M} \mathrm{A} \beta_{1-42}$ for $24 \mathrm{~h}$. Cells were allowed to equilibrate to RT for $~ 30$ min before the ATP assay was implemented. The luciferase-based solution was measured with a spectrophotometer (BioTek) by endpoint kinetic set to measure luminescence. ATP was estimated in nM per well using a standard curve. To prevent ATP degradation, the standard curve was created immediately prior to running the assays. The standard curve was prepared by serial tenfold dilutions of ATP in DMEM+.

\section{Mitochondrial Respiration Assay}

Mitochondrial respiration was measured using a Seahorse XFe (Seahorse Biosciences) bioanalyzer using the MitoStress Kit (Cat. No. 101706-100 (2018), Seahorse Bioscience) according to the manufacturer's guidelines. Cultured bEnd.3 cells were seeded on 96-well XF Cell Culture Microplates at a density of $1.5 \times 10^{4}$ cells per well and allowed to proliferate for 24h. Endothelial cells were exposed to 5-, 9-, and 18- $\mu \mathrm{M} \mathrm{A} \beta_{1-42}$ for $24 \mathrm{~h}$. Three hours prior to running the procedure, the sensor cartridges were hydrated and the instrument parameters set to run the assay. Endothelial cells were rinsed with XF assay medium and placed in the bioanalyzer for calibration. After calibration, each well was measured via the MitoStress Test protocol that used a base measurement of oxygen consumption ( $\mathrm{pmol} / \mathrm{min}$ ) to calculate basal respiration, maximal respiration, ATP production, spare capacity, proton leak, and nonmitochondrial respiration. 


\section{Cellular Calcium Assay}

Cytosolic and mitochondrial $\mathrm{Ca}^{2+}$ levels were measured with Fluo-4AM (Cat. No. F14201 (2018), Molecular Probes) or Rhod-2AM (Cat. No. R1245MP (2018), Molecular Probes) according to the manufacturer's guidelines, respectively. Endothelial cells were seeded on either 96 -well assay plates at a density of $1.5 \times 10^{4}$ or glass-bottom culture dishes at a density of $3.5 \times 10^{4}$ cells. Cells were exposed to 5-, 9-, and 18- $\mu \mathrm{M} \mathrm{A} \beta_{1-42}$ for $24 \mathrm{~h}$. Before conducting the experiment, cells were rinsed twice with DMEM+. To enhance dispersion of the $\mathrm{Ca}^{2+}$ indicators, an equal volume of dimethyl sulfoxide (DMSO) (Cat. No. D2650-100ML (2019), Sigma Aldrich) containing 20\% pluronic F-127 (Cat. No. P2443-1KG (2018), Sigma Aldrich) was added to the Fluo-4AM or Rhod-2AM stock solution. The Fluo-4AM or Rhod-2AM solution was diluted to $5 \mu \mathrm{M}$ in DMEM and added to each well. Cells were loaded for 45 min at $37{ }^{\circ} \mathrm{C}$. After loading, endothelial cells were washed 3 times with DMEM+ and allowed to incubate for an additional 30 min for complete de-esterification. Rhod-2AM was incubated for an additional $24 \mathrm{~h}$ to allow for mitochondrial (non-cytosolic) $\mathrm{Ca}^{2+}$ indication. Fluorescence was measured with a spectrophotometer (BioTek) by area scan routine using an excitation of 494 or 552 and an emission of 506 or 581 to measure Fluo-4AM or Rhod-2AM, respectively. Each well was measured in RFU and normalized to the average of the control group to produce a fold change value. For cells that were plated on glass-bottom culture dishes, dynamic $\mathrm{Ca}^{2+}$ indication was measured via confocal microscopy. Cells were imaged over a period of 94 min at $30 \mathrm{sec}$ intervals.

\section{Calcium Pathway Inhibitors}

The $\mathrm{IP}_{3} \mathrm{R}$ inhibitor, 2-aminoethoxydiphenylborate (2-APB) (Cat. No. 100065-100MG (2020), Sigma Aldrich) was prepared as a $100 \mathrm{mM}$ stock solution in DMSO; subsequent 
dilution to $50 \mu \mathrm{M}$ working concentration was prepared in DMEM+. The MCU antagonist, 4Quinolinecarboxamide (ER-000444793) (Cat. No. 50-193-1971 (2020), Fisher Scientific) was prepared as a $5 \mathrm{mM}$ stock solution in DMSO; working concentration was prepared in DMEM+ at $5 \mu \mathrm{M}$. The $\mathrm{mNCX}$ inhibitor, 2-[4-[(4-nitrophenyl)methoxy]phenyl]ethyl carbamimidothioate (KB-R7943) (Cat. No. 12-441-0 (2020), Fisher Scientific) stock solutions was prepared in DMSO at a concentration of $5 \mathrm{mM}$; subsequent dilution to $7 \mu \mathrm{M}$ working concentration was prepared in DMEM+. All compounds used in the following studies were prepared at working concentration immediately before use.

\section{Statistics}

All experiments were performed on either primary cerebrovascular endothelial cells from mice or the bEnd.3 cell line. Experiments involving isolated mitochondria were derived from primary cerebrovascular endothelial cells. Quantitative experiments were repeated a minimum of three times. Qualitative experiments were repeated a minimum of two times. Data are reported as the mean value of the experimental group across all replicates plus or minus the standard deviation (mean $\pm \mathrm{SD}$ ). The criteria for sample exclusion consisted of human error or instrument failure. No data was excluded from the statistical analysis of the study. Statistical comparison was performed on GraphPad Prism version 8.0 (RRID: SCR_002798) by one-way ANOVA (Supplemental Table 1) to assess normality of data and with either Bonferroni's multiple comparison analysis (to compare each group to all other groups), Dunnett's post hoc analysis (to compare all groups to control group), or linear trend analysis (linear trend to determine dose-dependency) (Supplemental Table 2). All p-values less than 0.05 were considered significant $(*, \mathrm{p}<0.05 ; * *, \mathrm{p}<0.001 ; * * *, \mathrm{p}<0.0001)$. 


\section{Results}

\section{Acute and chronic exposure to amyloid- $\beta$ is cytotoxic to cerebrovascular endothelial cells}

Previous studies have shown that, in neuronal cultures, exposure to $A \beta_{1-42}$ is cytotoxic, causing cellular death at relatively high concentrations (Ferreira et al. 2010; Takuma et al. 2004; Bastianetto et al. 2000). However, these experiments describe an unsustainable cellular environment at late stages of $\mathrm{AD}$, when $\mathrm{A} \beta_{1-42}$ is found at significantly elevated concentrations in the brain compared to the early stages of $\mathrm{AD}$. We constructed an experimental paradigm that describes the early changes to cells during the disease progression, when $A \beta_{1-42}$ is found at lower levels in the brain. To determine the cytotoxicity of acute exposure of $A \beta_{1-42}$ to cerebrovascular endothelial cells, we used brightfield microscopy to visualize live bEnd.3 cells exposed to $18 \mu \mathrm{M} A \beta_{1-42}$. We observed marked changes in cell morphology that resembled apoptosis, characterized by an initial retraction of cellular processes after 120 minutes that progressed and resulted in a spherical cellular morphology after only 240 minutes of exposure to $18 \mu \mathrm{M} A \beta_{1-42}$ (Figure 4.1A). To provide a quantitative measure of the bEnd.3 cell death we observed via live cell imaging, we counted the number of nuclei of cells exposed to 5-, 9-, and 18- $\mu \mathrm{M} A \beta_{1-42}$ for $24 \mathrm{~h}$ and compared this to the number of nuclei of bEnd.3 cells exposed to vehicle control. We found no significant difference in the number of nuclei between bEnd.3 cells that were exposed to $A \beta_{1-42}$ and cells that were not (Figure 4.1B). Although, we found some evidence of cytotoxicity for acute exposure to $A \beta_{1-42}$, we next evaluated whether prolonged exposure of cerebrovascular endothelial cells to $A \beta_{1-42}$ could induce cytotoxicity. We assessed this by exposing bEnd. 3 cells to $9 \mu \mathrm{M} A \beta_{1-42}$ over a period of $48-144 \mathrm{~h}$. In order to account for differences in the number of cells between the latency-groups, we normalized our cell death measurement by counting the total number of nuclei of both dead and live cells 
to produce a dead-to-live ratio $\left(\mathrm{F}_{\mathrm{D}} / \mathrm{F}_{\mathrm{L}}\right)$, and this value was converted to fold-change from the latency-matched non-exposed control group. We found that cell death caused by $9 \mu \mathrm{M} \mathrm{A} \beta_{1-42}$ begins after $96 \mathrm{~h}$ of exposure. Specifically, cell death was increased by $1.56 \pm 0.15(\mathrm{p}<0.0001)$ -fold and $1.52 \pm 0.21(\mathrm{p}<0.0001)$-fold by $96 \mathrm{~h}$ and $120 \mathrm{~h}$ of exposure, respectively (Figure 4.1C).

Cerebrovascular endothelial cell exposure to amyloid- $\beta$ results in mitochondrial dysregulation characterized by accelerated mitochondrial oxidative phosphorylation

Most cells respond to stress by increasing oxidative phosphorylation. For example, cellular damage originating from starvation (Mookerjee et al. 2017; Dias Zeidler et al. 2017), inflammation (Brace et al. 2016), membrane rupture (Yajima et al. 2009), thermal limitations (Jarmuszkiewicz et al. 2015; Downs and Heckathorn 1998), and ionic imbalance (Garlid and Paucek 2003; Pilchova et al. 2017) can result in increased oxidative phosphorylation, which may provide additional metabolic resources that are needed to repair damage incurred. We first assessed mitochondrial oxidative phosphorylation after exposure to $A \beta_{1-42}$. We used an extracellular flux bioanalyzer $\mathrm{XF}^{\mathrm{e}} 96$ to assess changes in mitochondrial oxygen consumption following $A \beta_{1-42}$ exposure. Oxygen consumption in cultured bEnd. 3 cell was measured for 14 min to obtain a baseline metabolic oxygen consumption rate. After $14 \mathrm{~min}$, bEnd. 3 cells were exposed to either $9 \mu \mathrm{M} A \beta_{1-42}$ or DMEM (control) for a total of $589 \mathrm{~min}$, to determine if $\mathrm{A} \beta_{1 \text { - }}$ 42 modulates mitochondrial oxygen consumption. Our analysis revealed a progressive increase 
A
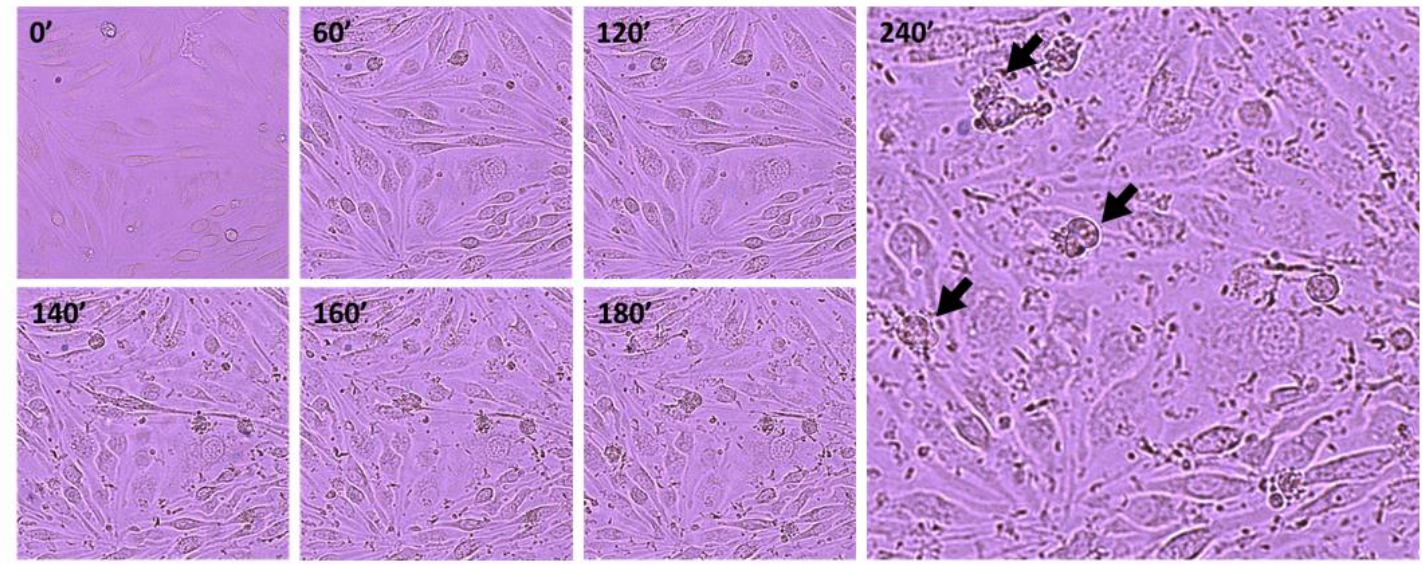

B
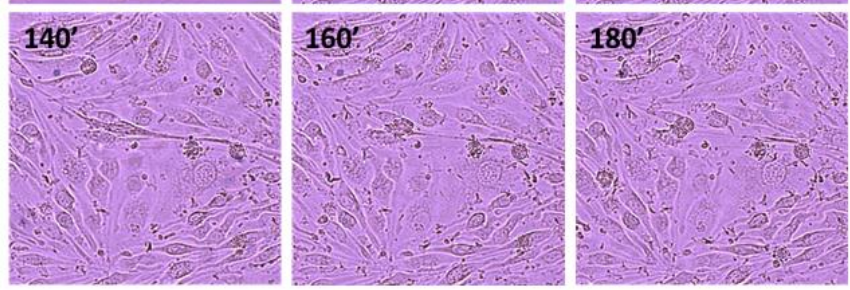

C
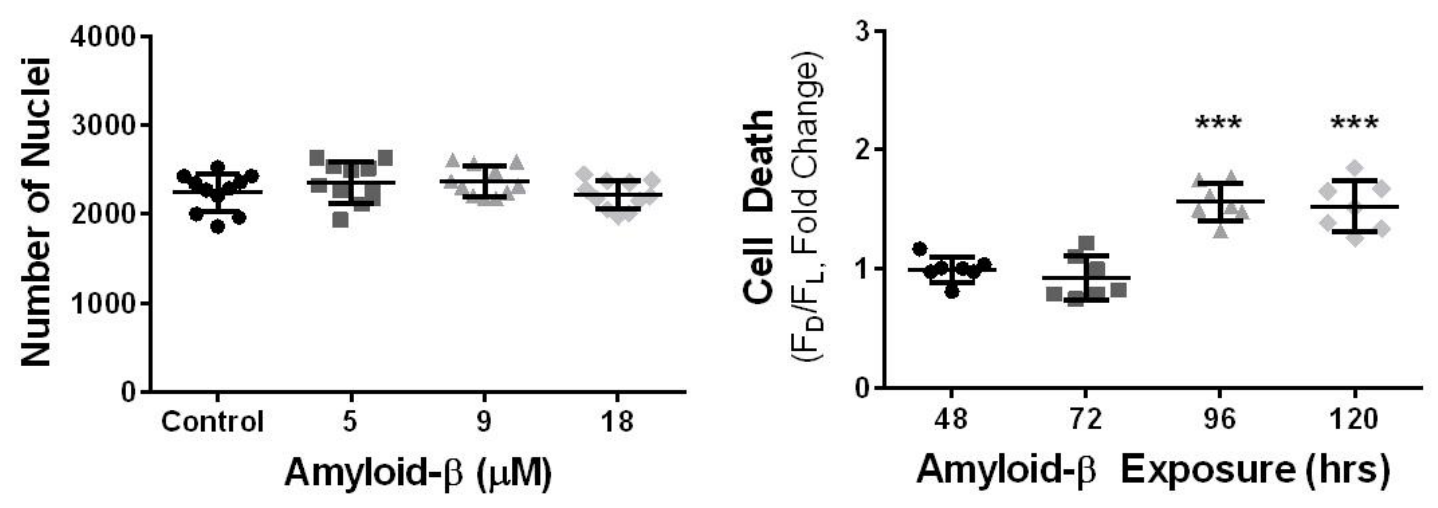

Figure 4.1. Cerebrovascular endothelial cell exposure to amyloid- $\beta$ results in cell death. (A) Brightfield micrographs depicting bEnd.3 cell death (black arrows) following exposure to $18 \mu \mathrm{M} A \beta_{1-42}$ for $0,60,120,140,160,180$, and 240 minutes. (B) Bar graph (mean $\pm \mathrm{SD}$ ) demonstrating the average number of nuclei per well after exposure to vehicle, $5 \mu \mathrm{MA} \beta_{1-42}, 9 \mu \mathrm{M} A \beta_{1-42}$, and $18 \mu \mathrm{M} \mathrm{A} \beta_{1-42}$ ( $\mathrm{n}=11$ wells per group). (C) Bar graph (mean \pm SD) depicting endothelial cell death after chronic exposure to $9 \mu \mathrm{M} \mathrm{A} \beta_{1-42}$ for $48,72,96$, 120 , and $144 \mathrm{~h}$ ( $\mathrm{n}=7$ wells per group). One-way ANOVA with Dunnett's post analysis was used to determine the level of significance between the exposure groups (***, $p<0.0001)$. 
in mitochondrial oxygen consumption that began 397 min post- $\mathrm{A} \beta_{1-42}$ exposure, which increased $1.81 \pm 1.77$-fold $(\mathrm{p}<0.001)$ above the control group after $461 \mathrm{~min}$ and continued to increase up to $3.76 \pm 1.41$-fold $(\mathrm{p}<0.001)$ above the control group over the duration of the experiment (Figure 4.2A). These observations were in contrast to bEnd.3 cells maintained in DMEM without $A \beta_{1-42}$, which demonstrated an essentially unchanged oxygen consumption rate for the duration of the experiment (Figure 4.2A, black).

Since our results demonstrated a clear increase in mitochondrial oxygen consumption in response to $A \beta_{1-42}$ exposure, we characterized specific indices of mitochondrial oxygen consumption. To determine this, we measured cellular basal respiration, maximum respiration, spare capacity, and proton leak after bEnd.3 cells were exposed for $24 \mathrm{~h}$ to 5-, 9-, and 18- $\mu \mathrm{M}$ $A \beta_{1-42}$. We found that $A \beta_{1-42}$ dose-dependently increased mitochondrial basal respiration, maximal respiration, spare capacity, and proton leak after $24 \mathrm{~h}$ of exposure. For all evaluated parameters of oxidative phosphorylation, the maximum effect was measured for bEnd.3 cells exposed to $18 \mu \mathrm{M} A \beta_{1-42}$. Specifically, exposure to $A \beta_{1-42}$ resulted in an increased oxygen consumption to $67 \pm 5.6(\mathrm{p}<0.0001), 159 \pm 13.5(\mathrm{p}<0.0001), 88 \pm 14.9(\mathrm{p}<0.0001)$, and 15 $\pm 1.6(\mathrm{p}<0.0001) \mathrm{pmol} / \mathrm{min}$ of oxygen used for basal respiration, maximum respiration, spare capacity, and proton leak, respectively (Figure 4.2B-E).

We then repeated this experiment on primary cerebrovascular endothelial cells to determine if $A \beta_{1-42}$ exposure results in similar mitochondrial bioenergetic hyperactivity. We observed a similar dose-dependent upregulation of mitochondrial oxygen consumption. The maximum effect was measured from cells exposed to $18 \mu \mathrm{M} A \beta_{1-42}$, which caused oxygen consumption to increase to $167 \pm 11.2(\mathrm{p}<0.0001), 311 \pm 45.3(\mathrm{p}<0.0001), 144 \pm 44.9(\mathrm{p}<$ $0.001)$, and $48 \pm 11.5(\mathrm{p}<0.0001) \mathrm{pmol} / \mathrm{min}$ of oxygen used for basal respiration, maximum 
respiration, spare capacity, and proton leak, respectively (Figure 4.2F-I). Thus, both primary cerebrovascular endothelial cells and bEnd.3 cells responded to $A \beta_{1-42}$ by increasing cellular respiration in a dose-dependent manner.

\section{Amyloid- $\beta$ exposure results in an increased mitochondrial $\mathrm{Ca}^{2+}$ concentration}

It is firmly established that mitochondrial activity is modulated by $\mathrm{Ca}^{2+}$. Since mitochondrial oxygen consumption was increased in both primary cerebrovascular endothelial cells and in bEnd. 3 cells that were exposed to $A \beta_{1-42}$, we hypothesized that mitochondrial $\mathrm{Ca}^{2+}$ dyshomeostasis participated in the $\mathrm{A} \beta_{1-42}$-induced mitochondrial dysregulation. We next measured mitochondrial matrix $\mathrm{Ca}^{2+}$ concentration in bEnd.3 cells after exposure to 5-, 9-, and 18- $\mu \mathrm{M}$ of $\mathrm{A} \beta_{1-42}$ for $24 \mathrm{~h}$ and compared this to the $\mathrm{Ca}^{2+}$ concentration in cells that were not exposed to $A \beta_{1-42}$. We found a dose-dependent increase of mitochondrial matrix $\mathrm{Ca}^{2+}$ concentrations after $24 \mathrm{~h}$ exposure to $\mathrm{A} \beta_{1-42}$. Compared to vehicle-treated controls $(100 \%)$, our data demonstrated that $A \beta_{1-42}$ increased mitochondrial $\mathrm{Ca}^{2+}$ levels to $119 \pm 30.1 \%(\mathrm{p}<0.001)$ at $9 \mu \mathrm{M} \mathrm{A} \beta_{1-42}$ and $153 \pm 24.7 \%(\mathrm{p}<0.0001)$ at $18 \mu \mathrm{M} \mathrm{A} \beta_{1-42}$ (Figure 4.3A).

Because mitochondrial uptake of $\mathrm{Ca}^{2+}$ is a critical process required to clear cytosolic $\mathrm{Ca}^{2+}$, we assessed the concentration of cytosolic $\mathrm{Ca}^{2+}$ after exposure to $\mathrm{A} \beta_{1-42}$. Cytosolic $\mathrm{Ca}^{2+}$ was measured via Fluo-4AM fluorescence intensity after exposure to 5-, 9-, and 18- $\mu \mathrm{M} A \beta_{1-}$ ${ }_{42}$ for $24 \mathrm{~h}$. We found a dose-dependent decrease in cytosolic $\mathrm{Ca}^{2+}$ concentration following $\mathrm{A} \beta_{1-}$ 42 exposure. In bEnd. 3 cells exposed to $18 \mu \mathrm{M} A \beta_{1-42}$, cytosolic $\mathrm{Ca}^{2+}$ declined to nearly $64 \pm$ $4.9 \%(\mathrm{p}<0.0001)$ of the $\mathrm{Ca}^{2+}$ measured in non-exposed cells (Figure 4.3B). 
A
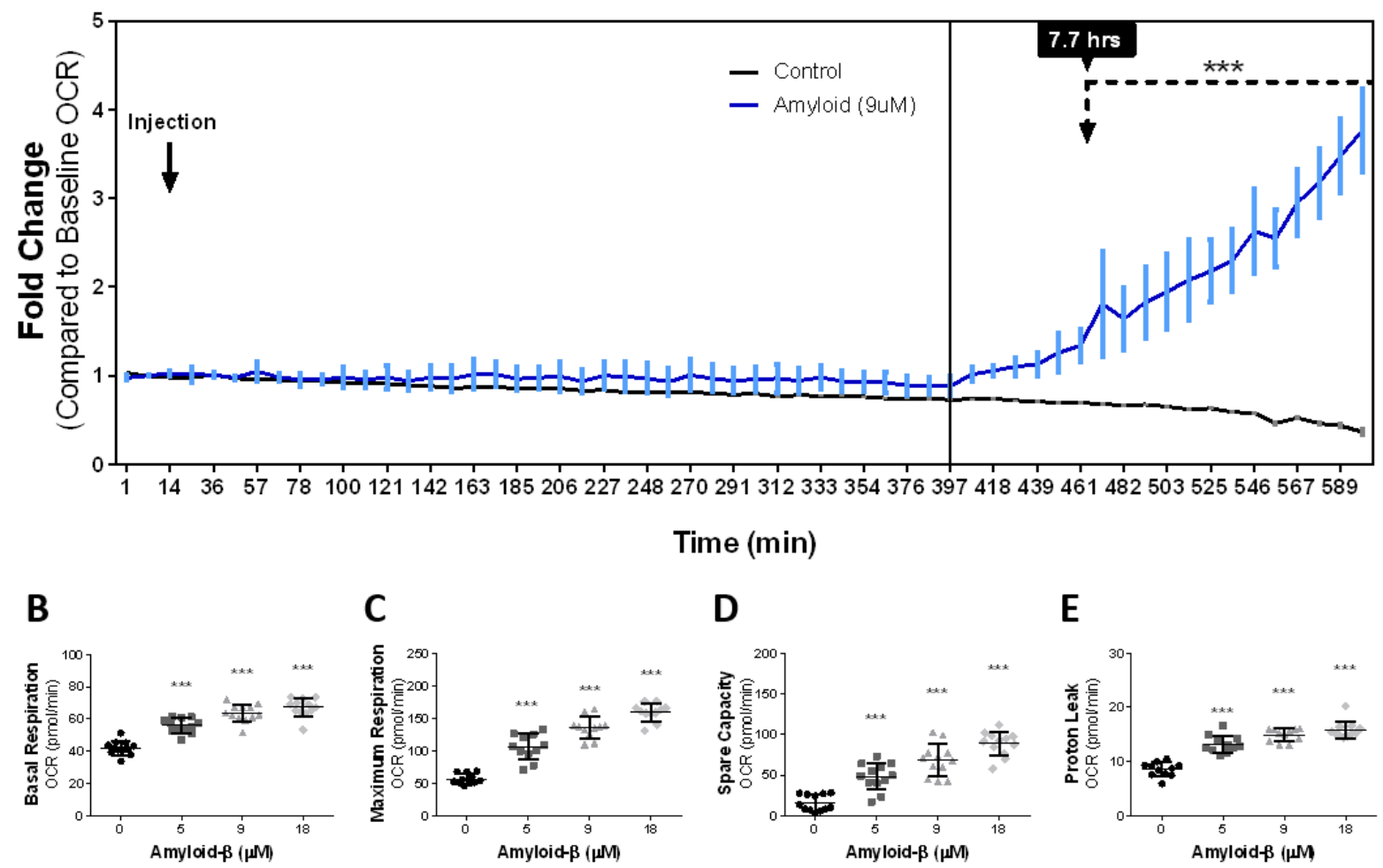

D

$\mathbf{E}$
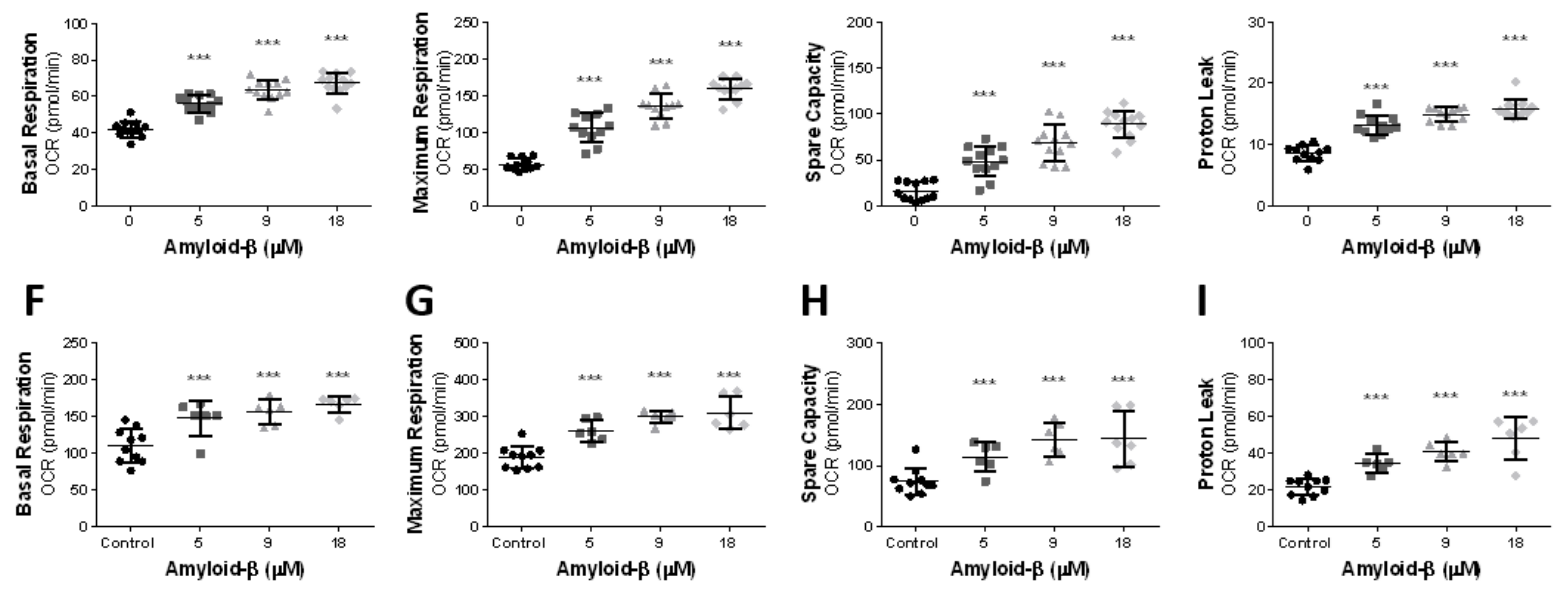

Figure 4.2. Exposure to amyloid- $\beta$ for 24-hours results in a dose-dependent increase in mitochondrial oxygen consumption. (A) Line graph (mean \pm SD) depicting the foldchange of oxygen consumption to baseline as a function of time from bEnd. 3 cells exposed to vehicle and $9 \mu \mathrm{M} \mathrm{A} \beta_{1-42}$. Bar graph(s) (mean $\pm \mathrm{SD}$ ) demonstrating oxygen consumption (pmol/min) by $(\mathbf{B}, \mathbf{F})$ basal respiration, $(\mathbf{C}, \mathbf{G})$ maximum respiration, $(\mathbf{D}, \mathbf{H})$ spare capacity, and (E, I) proton leak from (B-E) bEnd.3 cells and (F-H) primary cerebrovascular endothelial cells after $24 \mathrm{~h}$ exposure to vehicle, $5 \mu \mathrm{M} A \beta_{1-42}, 9 \mu \mathrm{M} \mathrm{A} \beta_{1-42}$, and $18 \mu \mathrm{M} \mathrm{A} \beta_{1-42}$. One-way ANOVA with Dunnett's post analysis and linear trend analysis was used to determine the level of significance between the treatment groups $(*, p<0.05 ; * *, p<0.001$; ***, $\mathrm{p}<0.0001),(\mathrm{B}-\mathrm{E}, \mathrm{n}=12$ wells per group; F-I, control = 10, all other conditions $=6$ wells per group). 


\section{Cellular ATP content increases dose-dependently following exposure to $A_{\beta_{1-42}}$}

Cellular ATP production is stringently coupled to the cell's ATP requirements. Following the observation, that $\mathrm{A} \beta_{1-42}$ exposure leads to mitochondrial $\mathrm{Ca}^{2+}$ accumulation and because intra-mitochondrial $\mathrm{Ca}^{2+}$ can modulate ATP production, we questioned whether changes to ATP production result from exposure to $A \beta_{1-42}$. We evaluated mitochondrial ATP production by calculating the oxygen consumption used to produce ATP by summing the oxygen consumed by proton leak and mitochondrial respiration then subtracting this value from the oxygen consumed by the cell's basal respiration. This produced an estimate for oxygen in $\mathrm{pmol} / \mathrm{min}$ that was used for ATP production. Our analysis indicated that cells exposed to $A \beta_{1-42}$ dose-dependently increased ATP production after 24 hours of exposure. bEnd. 3 cells exposed to $18 \mu \mathrm{M} \mathrm{A} \beta_{1-42}$ consumed as much as $51 \pm 4.6(\mathrm{p}<0.0001) \mathrm{pmol} / \mathrm{min}$ of oxygen for ATP production compared to only $33 \pm 3.0 \mathrm{pmol} / \mathrm{min}$ of oxygen by non-exposed cells (Figure 4.4A).

We next repeated the experiment on primary cerebrovascular endothelial cells to determine if $A \beta_{1-42}$ mediates similar alterations to oxygen consumption for ATP production. Primary cerebrovascular endothelial cells demonstrated a dose-dependent increase of oxygen consumed for ATP production. Specifically, cells exposed to $9 \mu \mathrm{M}$ and $18 \mu \mathrm{M} A \beta_{1-42}$ consumed $121 \pm 13.8(\mathrm{p}<0.05)$ and $130 \pm 7.6(\mathrm{p}<0.0001) \mathrm{pmol} / \mathrm{min}$ of oxygen, respectively (Figure 4.4B). These data indicate that primary cerebrovascular endothelial cells respond to $\mathrm{A} \beta_{1-42}$ exposure similarly to bEnd. 3 cells. 


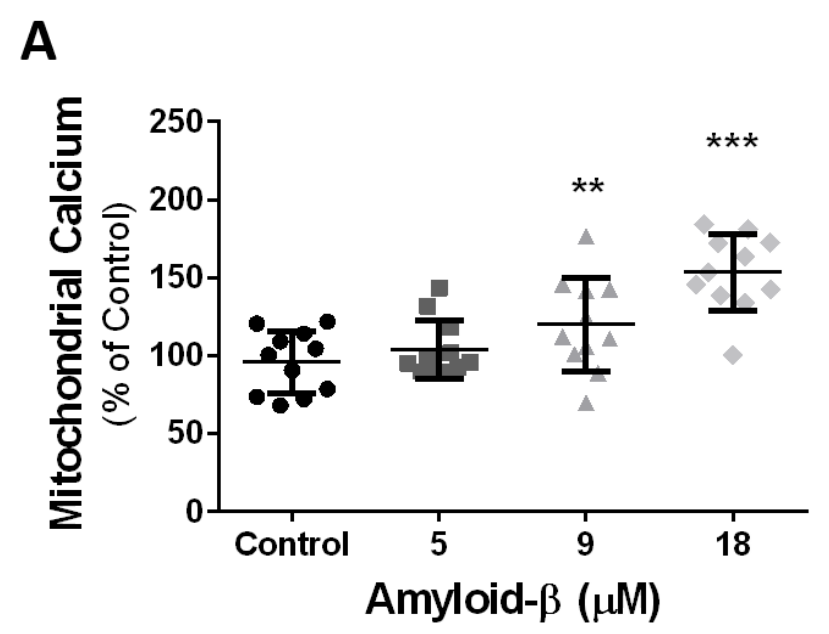

B

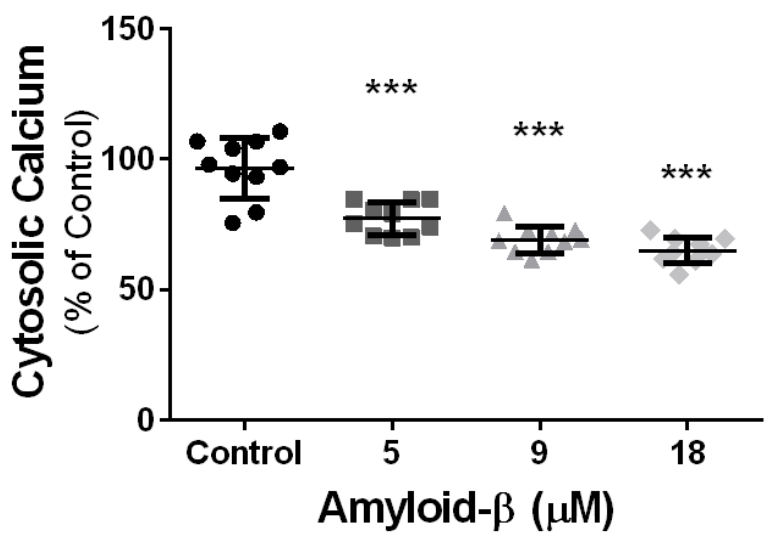

Figure 4.3. Exposure to amyloid- $\beta$ for 24 -hours causes a dose-dependent increase in mitochondrial $\mathrm{Ca}^{2+}$ content and a dose-dependent decrease of cytosolic $\mathrm{Ca}^{2+}$. (A) Bar graph (mean $\pm \mathrm{SD}$ ) depicting mitochondrial $\mathrm{Ca}^{2+}$ content as percentage of vehicle treated cells, after $24 \mathrm{~h}$ exposure to $5 \mu \mathrm{M} \mathrm{A} \beta_{1-42}, 9 \mu \mathrm{M} A \beta_{1-42}$, and $18 \mu \mathrm{MA} \beta_{1-42}(\mathrm{n}=11$ wells per group). (B) Bar graph (mean $\pm \mathrm{SD}$ ) demonstrating cytosolic $\mathrm{Ca}^{2+}$ content as percentage of vehicle treated cells after $24 \mathrm{~h}$ exposure to $5 \mu \mathrm{M} \mathrm{A} \beta_{1-42}, 9 \mu \mathrm{M} \mathrm{A} \beta_{1-42}$, and $18 \mu \mathrm{MA} \beta_{1-42}(\mathrm{n}=$ 10 wells per group). One-way ANOVA with Dunnett's post analysis and linear trend analysis was used to determine the level of significance between the treatment groups (**, $\mathrm{p}=0.001 ; * * *, \mathrm{p}=0.0001)$. 
An increase in mitochondrial oxidative phosphorylation is often indicative of a cellular ATP deficit. This may result from cellular injury, increased cellular activity, or as a response to cell signaling. Because we observed an increase in mitochondrial oxygen consumption for ATP production, we next measured cellular ATP content in bEnd. 3 cells to determine if the changes in oxygen consumption reflect ATP availability. We found that $24 \mathrm{~h}$ of exposure to $\mathrm{A} \beta_{1-42}$ resulted in a dose-dependent increase in intracellular ATP content. Exposure to $18 \mu \mathrm{M}$ $\mathrm{A} \beta_{1-42}$ resulted in an increase in the intracellular ATP concentration to $1922 \pm 41.4(\mathrm{p}<0.0001)$ $\mathrm{nM}$ compared to $1683 \pm 46.2 \mathrm{nM}$ in cells that were not exposed to $\mathrm{A} \beta_{1-42}$ (Figure $4.4 \mathrm{C}$ ). Interestingly, the dose-dependent increase of intracellular ATP content occurred alongside a dose-dependent decrease in extracellular ATP. We found that the medium from cell that were not exposed to $A \beta_{1-42}$ contained $7.8 \pm 1.3 \mathrm{nM}$ of ATP which decreased as low as $5.6 \pm 0.5$ (p $<0.0001) \mathrm{nM}$ of ATP in the medium from cell exposed to $18 \mu \mathrm{M} \mathrm{A} \beta_{1-42}$ (Figure 4.4D). These data suggest that dysregulated mitochondria metabolism is characterized by aberrant ATP production that surpasses ATP utilization.

\section{Mitochondrial superoxide production is driven by amyloid- $\beta$-induced acceleration of mitochondrial oxygen consumption}

Mitochondrial production of superoxide is the primary contributor to the oxidative damage during aging and degenerative diseases (Brand et al. 2004). We next assessed whether the $\mathrm{A} \beta_{1-42}$-induced increase in mitochondrial oxygen consumption in both primary cerebrovascular endothelial cells and bEnd. 3 cells results in elevated mitochondrial superoxide production. Mitochondrial superoxide was probed with a fluorescence indicator after bEnd.3 

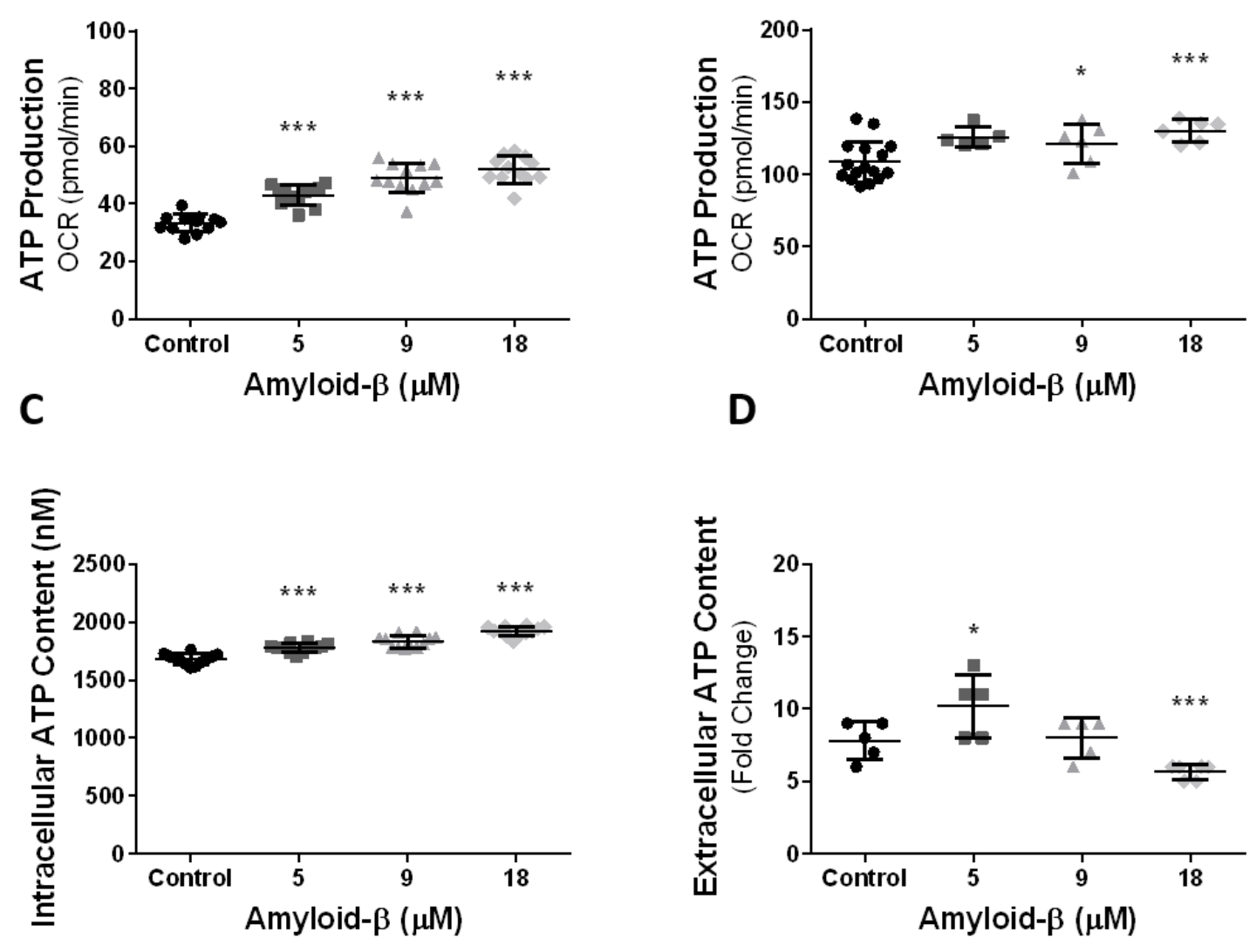

Figure 4.4. Exposure to amyloid- $\beta$ causes a dose-dependent increase in cellular ATP production and cytosolic ATP content. Bar graph(s) (mean \pm SD) depicting mitochondrial oxygen consumption ( $\mathrm{pmol} / \mathrm{min}$ ) for ATP production in (A) bEnd.3 ( $\mathrm{n}=12$ wells per group) and (B) primary cerebrovascular endothelial cells after 24h exposure to vehicle control, $5 \mu \mathrm{M} \mathrm{A} \beta_{1-42}, 9 \mu \mathrm{MA} \beta_{1-42}$, and $18 \mu \mathrm{MA} \beta_{1-42}$ (control = 15, else $=6$ wells per group). Bar graph(s) (mean $\pm \mathrm{SD}$ ) demonstrating the $(\mathbf{C})$ intracellular $(\mathrm{n}=12$ wells per group) and (D) extracellular ( $\mathrm{n}=5$ wells per group) ATP content ( $\mathrm{nM}$ ) in bEnd. 3 cells after exposure to vehicle control, $5 \mu \mathrm{M} \mathrm{A} \beta_{1-42}, 9 \mu \mathrm{M} A \beta_{1-42}$, and $18 \mu \mathrm{M} A \beta_{1-42}$. Statistical analysis was performed by One-way ANOVA with Dunnett's post analysis and linear trend analysis to determine the level of significance $\left(*, p=0.01 ; *^{*}, p=0.001 ; * * *, p=0.0001\right)$. 
cells were exposed to $A \beta_{1-42}$ for $24 \mathrm{~h}$. The fluorescence intensity of the superoxide indicator was converted into fold-change relative to vehicle treated cells. Cultured bEnd.3 cells demonstrated a remarkable dose-dependent increase in mitochondrial superoxide production following $24 \mathrm{~h}$ exposure to $A \beta_{1-42}$. Mitochondrial superoxide production increased $9.2 \pm 3.9$ $(\mathrm{p}<0.0001)-$ fold and $12.1 \pm 3.8(\mathrm{p}<0.0001)-$ fold above vehicle treated cells after $24 \mathrm{~h}$ exposure to $9 \mu \mathrm{M}$ and $18 \mu \mathrm{MA} \beta_{1-42}$, respectively (Figure 4.5D).

We next assessed the effects of increased extracellular $\mathrm{Ca}^{2+}$ on mitochondrial superoxide production and compared this with superoxide production following $A \beta_{1-42}$ exposure. To determine this, bEnd. 3 cells were probed with a fluorescent superoxide indicator then imaged via confocal microscope after $5 \mathrm{~min}$ to obtain a baseline and measurement (Figure 4.5A). After baseline measurement, cells were exposed to either $5 \mathrm{mM}$ of $\mathrm{Ca}^{2+}$ or $9 \mu \mathrm{M}$ of $\mathrm{A} \beta_{1 \text { - }}$ 42 and incubated for 30 min before obtaining an outcome measurement. Our time series image analysis revealed a $1.6 \pm 0.2(\mathrm{p}<0.0001)$-fold increase above baseline in mitochondrial superoxide production after 30 min exposure to $9 \mu \mathrm{M} A \beta_{1-42}$ (Figure 4.5B). Similarly, cells that were exposed to an additional $5 \mathrm{mM}$ of $\mathrm{Ca}^{2+}$ demonstrated a $1.4 \pm 0.1(\mathrm{p}<0.0001)-$ fold increase above baseline in superoxide production after $30 \mathrm{~min}$ of exposure (Figure 4.5C). These data suggest that increased $\mathrm{Ca}^{2+}$ concentration can drive superoxide production in bEnd. 3 cells at a magnitude similar to cells exposed to $A \beta_{1-42}$ for the same amount of time.

We were unsure if direct interaction between $A \beta_{1-42}$ and mitochondria produce the mitochondrial changes we observed. To answer this question, we isolated the mitochondria form primary cerebrovascular endothelial cells and measured superoxide production following $A \beta_{1-42}$ exposure for $1 \mathrm{~h}$. Isolated mitochondria exposed to $A \beta_{1-42}$ for $1 \mathrm{~h}$ resulted in a dosedependent increase in mitochondrial superoxide production. Exposure to 1-, 3-, 6-, and 9- $\mu \mathrm{M}$ 
$\mathrm{A} \beta_{1-42}$ demonstrated a $1.35 \pm 0.07(\mathrm{p}<0.001), 1.95 \pm 0.10(\mathrm{p}<0.0001), 3.10 \pm 0.13(\mathrm{p}<$ $0.0001)$, and $4.29 \pm 0.27(\mathrm{p}<0.0001)$-fold increase in superoxide production relative to control, respectively (Figure 4.5E).

\section{Amyloid- $\beta$ mediates mitochondrial dysregulation by increasing mitochondrial matrix $\mathrm{Ca}^{2+}$ that accelerates mitochondrial oxidative phosphorylation and superoxide production}

Mitochondrial influx of $\mathrm{Ca}^{2+}$ has been shown to accelerate oxidative phosphorylation and ATP production by increasing the activity of mitochondrial dehydrogenase enzymes. Therefore, we hypothesized that the increased oxygen consumption and production of superoxide by cerebrovascular endothelial cell mitochondria following exposure to $A \beta_{1-42}$ is mediated by the $\mathrm{A} \beta_{1-42}$-induced $\mathrm{Ca}^{2+}$ accumulation in mitochondria. To determine if the increased $\mathrm{Ca}^{2+}$ in the mitochondrial matrix following the exposure of bEnd.3 cells to $A \beta_{1-42}$ mediates the hyperoxidative state of mitochondria, we reduced the $\mathrm{Ca}^{2+}$ available to mitochondria by chelation. Extracellular $\mathrm{Ca}^{2+}$ was chelated with $1 \mathrm{mM}$ ethylenediaminetetraacetic acid (EDTA) supplemented in cell culture medium with or without exposure to $A \beta_{1-42}$. We first assessed whether chelation of extracellular $\mathrm{Ca}^{2+}$ results in reduced $\mathrm{A} \beta_{1-42}$-induced mitochondrial $\mathrm{Ca}^{2+}$ accumulation. Cultured bEnd.3 cells were plated in 96-well assay plates at a density of $1.5 \times 10^{4}$ cells per well and grown for $24 \mathrm{~h}$. Cells were then exposed to either vehicle or $9 \mu \mathrm{MA} \beta_{1-42}$ with or without $1 \mathrm{mM}$ EDTA for $24 \mathrm{~h}$ and then assessed for changes in mitochondrial $\mathrm{Ca}^{2+}$ levels. Our results indicated that $1 \mathrm{mM}$ EDTA effectively abolished the $\mathrm{A} \beta_{1-42}$-induced accumulation of $\mathrm{Ca}^{2+}$ in the mitochondrial matrix (Figure 4.6A). Specifically, cells exposed to $9 \mu \mathrm{M}$ A $\beta_{1-42}$ without EDTA (9 $\mu \mathrm{M}$ A $\beta_{1-42} / 0$ mM EDTA) 
A
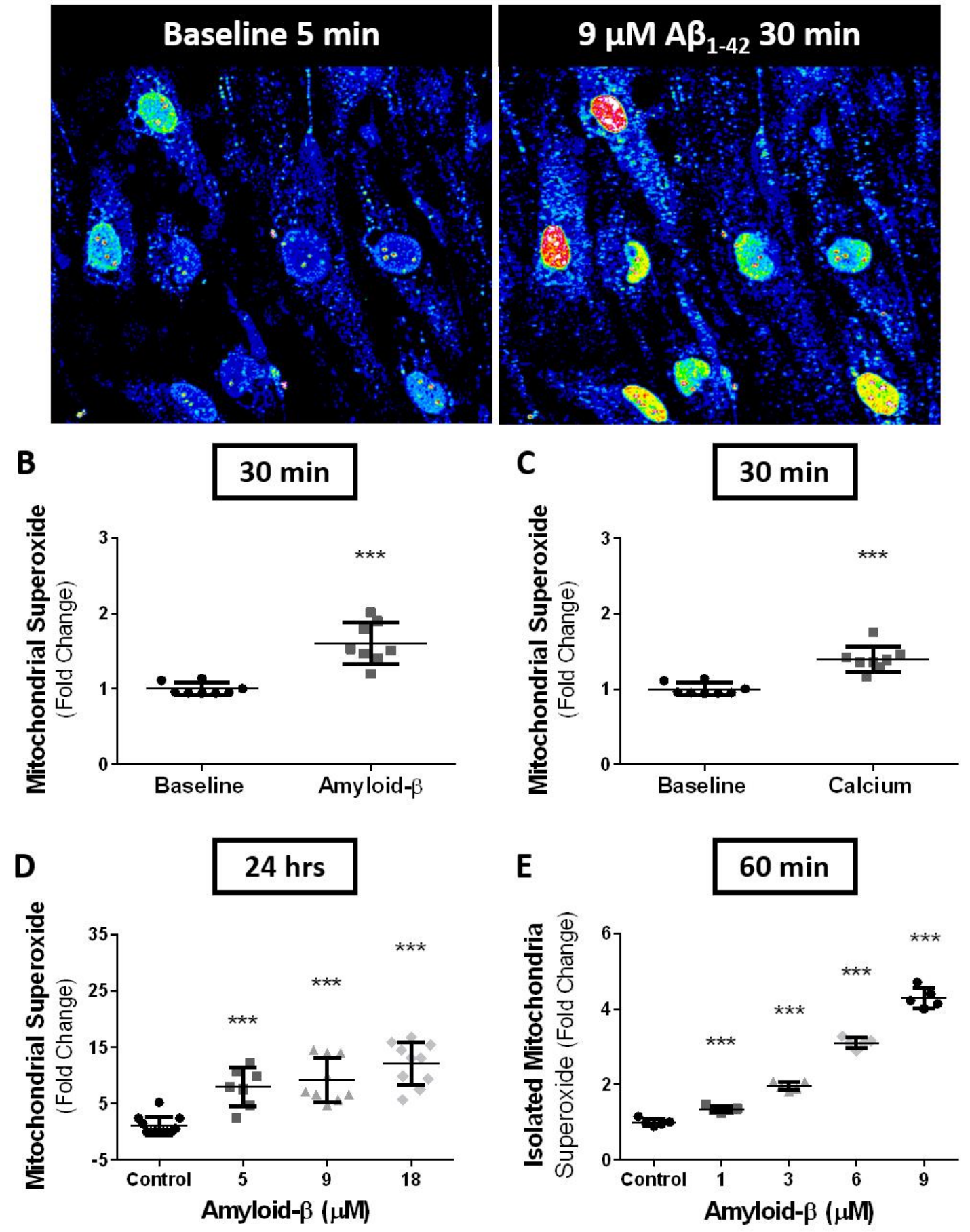
Figure 4.5. Exposure to amyloid- $\beta$ or $\mathrm{Ca}^{2+}$ results in an increase in mitochondrial superoxide production. (A) Time-lapse confocal micrographs depicting mitochondrial superoxide production in bEnd. 3 cells at baseline (left) and after $30 \mathrm{~min}$ of exposure to 9 $\mu \mathrm{M} \mathrm{A} \beta_{1-42}$ (right). Bar graph(s) (mean $\pm \mathrm{SD}$ ) demonstrating superoxide production from time-lapse images as fold-change from baseline for bEnd. 3 cells exposed to (B) $9 \mu \mathrm{M} \mathrm{A} \beta_{1}$ 42 ( $\mathrm{n}=8$ cells) and (C) $5 \mathrm{mM} \mathrm{CaCl}_{2}(\mathrm{n}=8$ cells) for 30 minutes. (D) Bar graph (mean \pm $\mathrm{SD})$ demonstrating mitochondrial superoxide production as fold-change in bEnd.3 cells after $24 \mathrm{~h}$ of exposure to vehicle, $5 \mu \mathrm{M} \mathrm{A} \beta_{1-42}, 9 \mu \mathrm{M} \mathrm{A} \beta_{1-42}$, and $18 \mu \mathrm{M} \mathrm{A} \beta_{1-42}(\mathrm{n}=10$ wells per group). (E) Bar graph (mean $\pm \mathrm{SD}$ ) depicting superoxide production as fold-change by isolated mitochondria from primary cerebrovascular endothelial cells after $1 \mathrm{~h}$ exposure to $0,1,3,6$, and $9 \mu \mathrm{M} \mathrm{A} \beta_{1-42}(\mathrm{n}=5$ wells per group). Statistical analysis was performed by one-way ANOVA with Dunnett's post analysis and linear trend analysis to determine the level of significance $(* * *, p=0.0001)$. 
increased mitochondrial $\mathrm{Ca}^{2+}$ to $1.8 \pm 0.4$-fold (vs. control, $\mathrm{p}<0.0001$ ) above cells exposed to vehicle (control). Whereas, cells exposed to $9 \mu \mathrm{M} \mathrm{A} \beta_{1-42}$ with $1 \mathrm{mM}$ EDTA (9 $\mu \mathrm{M} \mathrm{A} \beta_{1-42}$ / 1 mM EDTA) completely abolished the $\mathrm{A} \beta_{1-42}$-induced accumulation of mitochondrial $\mathrm{Ca}^{2+}$ to $0.9 \pm 0.4($ mean $\pm \mathrm{SD})-$ fold (vs. $\mathrm{A} \beta$ only, $\mathrm{p}<0.0001)$ compared to control cells $(\mathrm{p}=0.65)$ (Figure 4.6A). These data indicate that extracellular $\mathrm{Ca}^{2+}$ chelation with EDTA is effective at reducing mitochondrial $\mathrm{Ca}^{2+}$.

We next assessed the effectiveness of reducing mitochondrial $\mathrm{Ca}^{2+}$ accumulation with EDTA at ameliorating elevated mitochondrial oxygen consumption after exposure to $A \beta_{1-42}$. To determine this, we measured the oxygen consumption rate of bEnd. 3 cells exposed to either 0 or $9 \mu \mathrm{M} \mathrm{A} \beta_{1-42}$ with or without $1 \mathrm{mM}$ EDTA. As before, cells exposed to $9 \mu \mathrm{M} \mathrm{A} \beta_{1-42}$ without EDTA for 24h demonstrated increased oxygen consumption by basal respiration $(114 \pm 4.0$ $\mathrm{pmol} / \mathrm{min}, \mathrm{p}<0.0001)$, maximum respiration $(296 \pm 8.4 \mathrm{pmol} / \mathrm{min}, \mathrm{p}<0.0001)$, and spare capacity $(182 \pm 8.0 \mathrm{pmol} / \mathrm{min}, \mathrm{p}<0.0001)$. However, treatment with $1 \mathrm{mM}$ EDTA completely ameliorated the $A \beta_{1-42}$-induced increase in oxygen consumption. In cells exposed to $9 \mu \mathrm{M}$ A $\beta_{1-42}$ with $1 \mathrm{mM}$ EDTA oxygen consumption returned to near vehicle control values for basal respiration $(101 \pm 3.3 \mathrm{pmol} / \mathrm{min}, \mathrm{p}<0.02)$, maximum respiration $(227 \pm 8.8 \mathrm{pmol} / \mathrm{min}, \mathrm{p}<$ $0.0001)$, and spare capacity $(126 \pm 7.2 \mathrm{pmol} / \mathrm{min}, \mathrm{p}<0.0001)$ (Figure 4.6B-D). Interestingly, proton leak was the only oxidative phosphorylation parameter that increased further with EDTA treatment. Cells exposed to $A \beta_{1-42}$ alone consumed $39 \pm 1.2(\mathrm{p}<0.0001) \mathrm{pmol} / \mathrm{min}$ of oxygen by proton leak while cells exposed to both $\mathrm{A} \beta_{1-42}$ and EDTA consumed $49 \pm 1.6(\mathrm{p}<$ 0.0001) $\mathrm{pmol} / \mathrm{min}$ of oxygen (Figure 4.6E). In support of our hypothesis, reduction of mitochondrial $\mathrm{Ca}^{2+}$ ameliorates the increased oxygen consumption mediated by mitochondrial 
$\mathrm{Ca}^{2+}$ accumulation following exposure to $A \beta_{1-42}$. These data suggest that $A \beta_{1-42}$ exposure results in mitochondrial dysfunction by increasing mitochondrial $\mathrm{Ca}^{2+}$ levels.

Mitochondrial $\mathrm{Ca}^{2+}$ content is stimulatory to ATP synthesis by the ATP synthase respiratory complex. Therefore, we predicted that by blocking $\mathrm{Ca}^{2+}$ from entering mitochondria, the $\mathrm{A} \beta_{1-42}$-induced upregulation of ATP production should be prevented. To determine the interplay between elevated mitochondrial oxygen consumption and matrix $\mathrm{Ca}^{2+}$ accumulation on the production of ATP, we exposed bEnd.3 cells to $9 \mu \mathrm{M} A \beta_{1-42}$ with or without $1 \mathrm{mM}$ EDTA for $24 \mathrm{~h}$ and then estimated the production of ATP by mitochondria. Consistently, bEnd.3 cells exposed to $9 \mu \mathrm{M} A \beta_{1-42}$ responded by increasing oxygen consumption for ATP production to $75 \pm 3.5$ ( $\mathrm{p}<0.0001) \mathrm{pmol} / \mathrm{min}$. Oxygen consumption in cells that were exposed to $9 \mu \mathrm{M}$ A $\beta_{1-42}$ with $1 \mathrm{mM}$ EDTA restored ATP production to near vehicle control values, $51 \pm 2.6(\mathrm{p}<0.0001)$ (Figure 4.6F).

Since mitochondrial $\mathrm{Ca}^{2+}$ accelerates electron transport by increasing the activity of mitochondrial dehydrogenase enzymes, we hypothesized that blocking $A \beta_{1-42}$-induced mitochondrial $\mathrm{Ca}^{2+}$ accumulation would mitigate the increased production of superoxide by mitochondria following exposure to $A \beta_{1-42}$. To determine if mitochondrial $\mathrm{Ca}^{2+}$ accumulation mediates the $A \beta_{1-42}$-induced increase in mitochondrial superoxide production, we exposed bEnd.3 cells to $A \beta_{1-42}$ in the presence or absence of EDTA then measured mitochondrial superoxide levels. Superoxide production by bEnd.3 cells exposed to $9 \mu \mathrm{M} \mathrm{A} \beta_{1-42} / 0 \mathrm{mM}$ EDTA increased superoxide production $5.5 \pm 1.3$ (vs. control, $\mathrm{p}<0.0001$ ) -fold above control cells. Whereas, bEnd.3 cells exposed to $9 \mu \mathrm{M} \mathrm{A} \beta_{1-42} / 1 \mathrm{mM}$ EDTA reduced superoxide production to $1.8 \pm 0.2$ (vs. A $\beta$ only, $\mathrm{p}<0.0001$ ) -fold of control cells (Figure 4.6G). 
Mitochondrial fission-fusion dynamics are a transient process that serves a physiologically important function, i.e. regulation of mitochondrial quality control and metabolic homeostasis. Mitochondrial fragmentation often precedes mitophagy of damaged mitochondria that occurs during states of metabolic and oxidative stress (Quintana et al. 2018). Mitochondrial fragmentation is well-documented in AD animal models and in human AD patients (Wang et al. 2008; DuBoff et al. 2013). It is clear that oxidative stress can cause transient changes to the morphology of mitochondria while prolonged oxidation can result mitochondrial network fragmentation leading to apoptosis (Qi et al. 2011; Youle and Narendra 2011; Youle and van der Bliek 2012). Since our results demonstrated that chelation of extracellular $\mathrm{Ca} 2+$ was exceptionally effective at ameliorating the $\mathrm{A} \beta_{1-42}$-induced dysregulation of mitochondrial activity, we asked whether the chelation of extracellular $\mathrm{Ca}^{2+}$ can rescue mitochondria from aberrant fragmentation. We answered this by labeling mitochondria in bEnd.3 cells with MitoTracker CMXRos Red and the nuclei with DAPI counterstain following $24 \mathrm{~h}$ exposure to $9 \mu \mathrm{M}$ A $\beta_{1-42}$ with or without $1 \mathrm{mM}$ EDTA. Indeed, we observed fragmentation of the mitochondrial network in bEnd. 3 cells exposed to $A \beta_{1-42}$ alone and found that by chelation of extracellular $\mathrm{Ca}^{2+}$ completely prevented the morphological changes to the mitochondrial network (Figure 4.6H-K). These observations demonstrate the role of $\mathrm{A} \beta_{1-42}$-induced mitochondrial $\mathrm{Ca}^{2+}$ accumulation in the dysregulation of mitochondrial bioenergetics, resulting in mitochondrial fragmentation following oxidative damage.

Our data describes a mechanism in which mitochondrial $\mathrm{Ca}^{2+}$ accumulation following cell exposure to $A \beta_{1-42}$ mediates cell damage by dysregulating mitochondrial function. We next asked whether preventing the $A \beta_{1-42}$-induced mitochondrial dysfunction could rescue cerebrovascular endothelial cells from $\mathrm{A} \beta_{1-42}$ mediated death. To assess the cytoprotective 
effects of blocking $A \beta_{1-42}$-induced mitochondrial $\mathrm{Ca}^{2+}$ accumulation against $A \beta_{1-42}$ mediated cell death, we exposed bEnd. 3 cells to $9 \mu \mathrm{M} \mathrm{A} \beta_{1-42}$ with or without $1 \mathrm{mM}$ EDTA for $24 \mathrm{~h}$ and then imaged cellular changes in morphology. Exposure to either vehicle control (Figure 4.6L) or $1 \mathrm{mM}$ EDTA alone (Figure 4.6M) caused no apoptotic cells upon visualization. While, bEnd.3 cells that were exposed to $9 \mu \mathrm{M} \mathrm{A} \beta_{1-42} / 0 \mathrm{mM}$ EDTA (Figure $4.6 \mathrm{~N}$ and $\mathrm{P}$ ) demonstrated numerous apoptotic cells. However, bEnd. 3 cells that were exposed to $9 \mu \mathrm{M} A \beta_{1}$ 42 with $1 \mathrm{mM}$ EDTA (Figure 4.6O) completely prevented the apoptotic cells observed in cultures exposed to $A \beta_{1-42}$ alone. We observed a reduced number of apoptotic cells in cultures exposed to $A \beta_{1-42}$ with $1 \mathrm{mM}$ EDTA. We observed no apoptotic cells in cultures that were not exposed to $\mathrm{A} \beta_{1-42}$ with and without $1 \mathrm{mM}$ EDTA.

\section{Amyloid- $\beta$ associated calcium entry into the mitochondria occurs via multiple intracellular calcium regulation pathways}

Since we found that the $A \beta_{1-42}$-induced elevated mitochondrial calcium could be attenuated by extracellular calcium chelation, we next assessed intracellular calcium regulation pathways involved in calcium uptake by mitochondria. To assess the contribution of intracellular calcium regulation pathways we used pharmacological inhibitors, which prevent calcium entry into the mitochondria from the endoplasmic reticulum, mitochondrial permeability transition pore (mPTP), and mitochondrial membrane associated calcium exchangers. Mitochondrial calcium was measured with Rhod-2AM as previously stated, then exposed to $9 \mu \mathrm{M} \mathrm{A} \beta_{1-42}$ or DMEM+ control containing $50 \mu \mathrm{M}$ 2-APB, $5 \mu \mathrm{M}$ ER-000444793, $7 \mu \mathrm{M}$ KB-R7943, or vehicle (DMSO) for 24 hours. We found that bEnd. 3 cells exposed to 9 

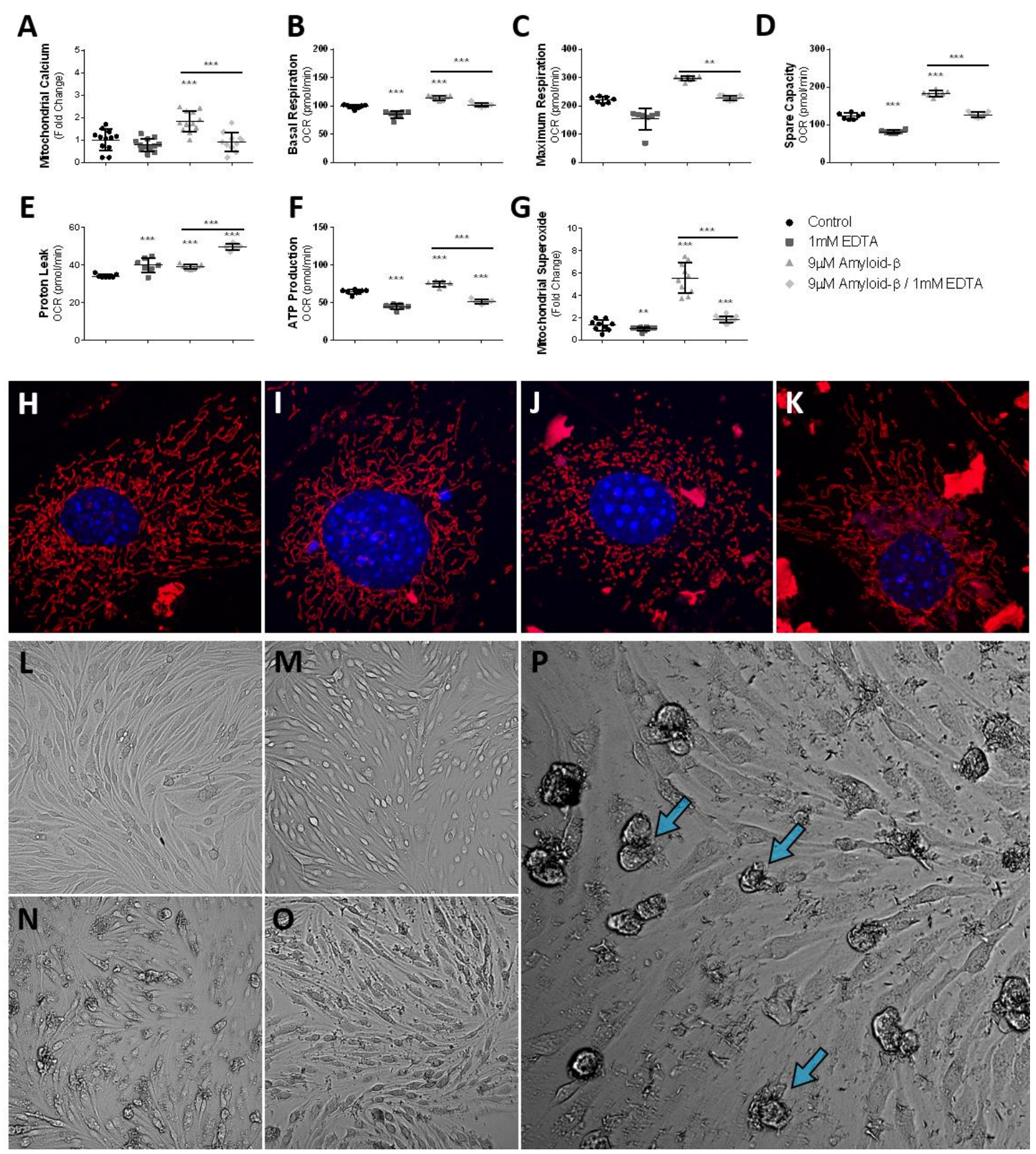
Figure 4.6. $\mathrm{Ca}^{2+}$ chelation reduces the amyloid- $\beta$ induced hyper-oxidative state of mitochondria and superoxide production via reduced mitochondrial $\mathrm{Ca}^{2+}$ uptake. (A) Bar graph (mean $\pm \mathrm{SD}$ ) demonstrating mitochondrial $\mathrm{Ca}^{2+}$ accumulation as fold-change in bEnd. 3 cells after $24 \mathrm{~h}$ exposure to vehicle control, 1 mM EDTA, $9 \mu \mathrm{M}$ A $\beta_{1-42}$, and $9 \mu \mathrm{M}$ $\mathrm{A} \beta_{1-42}$ with $1 \mathrm{mM}$ EDTA $(\mathrm{n}=12$ per group). Bar graph (mean $\pm \mathrm{SD}$ ) demonstrating mitochondrial oxygen consumption (pmol/min) by (B) basal respiration, (C) maximum respiration, (D) spare capacity, (E) proton leak, and (F) ATP production from bEnd.3 cells after $24 \mathrm{~h}$ exposure to vehicle control, $1 \mathrm{mM}$ EDTA, $9 \mu \mathrm{M}$ A $\beta_{1-42}$, and $9 \mu \mathrm{M}$ A $\beta_{1-42}$ with 1 $\mathrm{mM}$ EDTA ( $\mathrm{n}=7$ wells per group). (G) Bar graph (mean \pm SD) demonstrating mitochondrial superoxide production as fold-change in bEnd. 3 cells after $24 \mathrm{~h}$ exposure to vehicle control, $1 \mathrm{mM}$ EDTA, $9 \mu \mathrm{M} A \beta_{1-42}$, and $9 \mu \mathrm{M}$ A $\beta_{1-42}$ with $1 \mathrm{mM} \operatorname{EDTA}(\mathrm{n}=10$ wells per group). Confocal micrographs at 63x magnification depicting mitochondrial fragmentation (red) and nuclei (blue) in bEnd.3 cell exposed to $(\mathbf{H})$ vehicle, (I) $1 \mathrm{mM}$ EDTA, (J) $9 \mu \mathrm{M} \mathrm{A} \beta_{1-42}$, and (K) $9 \mu \mathrm{M} A \beta_{1-42}$ with 1 mM EDTA. Note mitochondria morphology depicted in panel $\mathbf{J}$, demonstrating numerous fragmented and punctated mitochondria while panel(s) H-I,K depict(s) complex and elongated mitochondria. Brightfield micrographs depicting bEnd.3 cell death (blue arrows) following exposure to 9 $\mu \mathrm{M} \mathrm{A} \beta_{1-42}$ after $24 \mathrm{~h}$ exposure to (L) vehicle control, (M) $1 \mathrm{mM}$ EDTA, (N, P) $9 \mu \mathrm{M}$ A $\beta_{1-}$ 42, and (O) $9 \mu \mathrm{M}$ A $\beta_{1-42}$ with 1 mM EDTA. One-way ANOVA with Dunnett's post analysis was used to determine significance compared to control while a student's 2-tailed t-test was used to compare the mean(s) of the $9 \mu \mathrm{M} \mathrm{A} \beta_{1-42}$ group and the $9 \mu \mathrm{M} A \beta_{1-42}$ with $1 \mathrm{mM}$ EDTA experimental group $(*, \mathrm{p}<0.05 ; * *, \mathrm{p}<0.001 ; * * *, \mathrm{p}<0.0001)$. 
$\mu \mathrm{M} \mathrm{A} \beta_{1-42}$-vehicle resulted in a $3.2 \pm 0.46(\mathrm{p}<0.0003)$-fold increase mitochondrial calcium above DMEM control $1 \pm 0.35$-fold. Whereas, treatment with the $\mathrm{IP}_{3}$ receptor antagonist 2APB $(50 \mu \mathrm{M})$ attenuated the elevated mitochondrial matrix calcium to $1.2 \pm 0.27$ ( $\mathrm{p}<0.00007)$ -fold. Inhibition of the mPTP with ER-000444973 $(5 \mu \mathrm{M})$ also reduced the $\mathrm{A} \beta_{1-42 \text {-induced }}$ mitochondrial calcium accumulation to $1.8 \pm 0.24(\mathrm{p}<0.002)$-fold. Treatment with an inhibitor of mitochondrial reverse mode $\mathrm{Na}^{+} / \mathrm{Ca}^{+}$exchanger and calcium uniporter, KB-R7943 $(7 \mu \mathrm{M})$ similarly reduced calcium in the mitochondrial matrix upon exposure to $A \beta_{1-42}$ to 1.4 $\pm 0.15(\mathrm{p}<0.0002)($ Figure 4.7)

\section{Discussion}

Although amyloid- $\beta$ is heavily studied in AD research, its precise role in the disease pathogenesis remains unknown. In AD brains, amyloid- $\beta$ plaques are found clustered around regions with damaged and degenerating mitochondria (Xie et al. 2013; Gillardon et al. 2007). In culture, amyloid- $\beta$ causes ROS production and cell death (Cho et al. 2009; Han et al. 2015). The mechanism that drives the amyloid- $\beta$-induced production of ROS is thought to involve the Nox subunit of NADPH oxidase that catalyzes the S-nitrosylation of cellular components (Miller et al. 2005; Drummond et al. 2011). S-nitrosylation of mitochondrial Drp-1 is shown to cause mitochondrial fission and may contribute to the mitochondrial fragmentation found in AD both in humans and in animal models (Cho et al. 2009). In addition, amyloid- $\beta$ has been shown to cause intracellular $\mathrm{Ca}^{2+}$ dyshomeostasis in culture (LaFerla 2002). However, the interplay between amyloid- $\beta$ induced ROS production, mitochondrial effects, and intracellular $\mathrm{Ca}^{2+}$ levels has not been elucidated previously. 


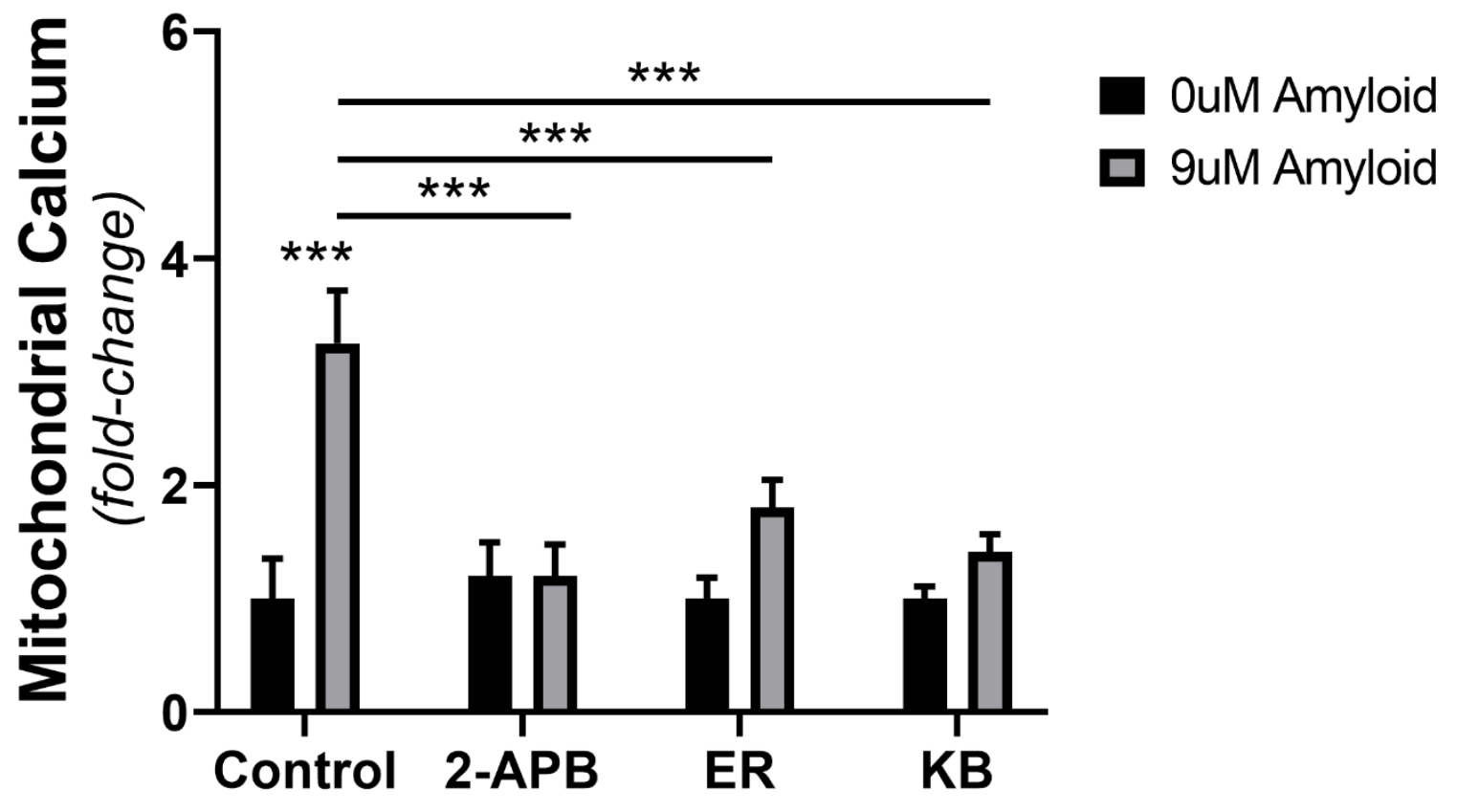

Figure 4.7. Antagonism of regulatory intracellular calcium pathways attenuates the A $\beta$-induced mitochondrial calcium influx. Bar graph (mean $\pm \mathrm{SD}$ ) demonstrating mitochondrial $\mathrm{Ca}^{2+}$ accumulation as fold-change in bEnd.3 cells after 24h exposure to vehicle control, $9 \mu \mathrm{M} \mathrm{A} \beta_{1-42}$, and $9 \mu \mathrm{M} \mathrm{A} \beta_{1-42}$ with either $50 \mu \mathrm{M}$ 2-APB, $5 \mu \mathrm{M}$ ER000444793 , or $7 \mu$ M KB-R7943 ( $n=12$ per group). Student's 2-tailed t-test was used to compare the mean(s) of each experimental group $(* * *, p<0.0001)$. 
We demonstrate in brain vascular endothelial cells that exposure to amyloid- $\beta$ causes a rapid change in cellular bioenergetics, characterized by accelerated mitochondrial oxygen consumption and increased superoxide production. We find that these changes were concurrent with the accumulation of calcium in mitochondria and could be prevented by blocking calcium from entering mitochondria by chelation.

In the present study, we investigate the early changes to endothelial cells following exposure to amyloid- $\beta$ that contribute to the cellular death observed after prolong exposure to the peptide. We noticed a rapid change to mitochondrial oxygen consumption after 7 hours of exposure to amyloid- $\beta$, and in both bEnd. 3 cells and in primary cerebrovascular endothelial cells, amyloid- $\beta$ exposure increased basal respiration, maximum respiration, spare capacity, and proton leak by 24 hours. Mitochondrial basal respiration is strongly influenced by the turnover of ATP and partially by the oxidation of substrates and by the leakage of protons from the intermembrane space (Brown et al. 1990; Ainscow and Brand 1999). Therefore, the rate of basal respiration reflects the cellular demand for ATP. In the present study, elevated basal respiration may indicate an increased endothelial demand for ATP in response to the early cellular injury following exposure to amyloid- $\beta$. The spare respiratory capacity of a cell is defined by the ability to utilize substrate and electron transport to meet cellular energy demand before reaching its biological limit (Yadava and Nicholls 2007; Choi et al. 2009). We observed a dose-dependent increase in spare respiratory capacity for both bEnd.3 cells and primary cerebrovascular endothelial cells. Because we observed both an elevated basal respiration and spare respiratory capacity in cells exposed to amyloid- $\beta$, we interpret this to suggest that both an enhanced mitochondrial respiratory competency and an elevated respiratory rate participate in the mechanism of amyloid- $\beta$-induced cellular injury. 


\section{Dose-dependent Response to Amyloid- $\beta$}

\begin{tabular}{|c|c|c|c|c|}
\hline Assessment & Subject & $\begin{array}{c}\text { Correlation } \\
\text { Coefficient } \\
\left(\mathrm{R}^{2}\right)\end{array}$ & $\begin{array}{l}\text { Linear Trend } \\
\text { (p-value) }\end{array}$ & $\begin{array}{c}\text { Data Depiction } \\
\text { (Figure, Panel) }\end{array}$ \\
\hline Basal Respiration & bEnd.3 & 0.4554 & $<0.0001$ & Fig. 4.2B \\
\hline Maximum Respiration & bEnd.3 & 0.5784 & $<0.0001$ & Fig. $4.2 \mathrm{C}$ \\
\hline Spare Capacity & bEnd.3 & 0.4988 & $<0.0001$ & Fig. 4.2D \\
\hline Proton Leak & bEnd.3 & 0.2811 & 0.0009 & Fig. 4.2E \\
\hline Basal Respiration & Primary Endothelial & 0.1843 & 0.0853 & Fig. $4.2 \mathrm{~F}$ \\
\hline Maximum Respiration & Primary Endothelial & 0.3054 & 0.0193 & Fig. $4.2 \mathrm{G}$ \\
\hline Spare Capacity & Primary Endothelial & 0.1327 & 0.1419 & Fig. $4.2 \mathrm{H}$ \\
\hline Proton Leak & Primary Endothelial & 0.3646 & 0.0103 & Fig. 4.21 \\
\hline Mitochondrial Calcium & bEnd. 3 & 0.241 & 0.004 & Fig. 4.3A \\
\hline Cytosolic Calcium & bEnd.3 & 0.223 & 0.0027 & Fig. $4.3 \mathrm{~B}$ \\
\hline ATP Production & bEnd.3 & 0.4083 & $<0.0001$ & Fig. $4.4 \mathrm{~A}$ \\
\hline ATP Production & Primary Endothelial & 0.2028 & 0.0104 & Fig. 4.4B \\
\hline ATP Content, Intracellular & bEnd. 3 & 0.6478 & $<0.0001$ & Fig. $4.4 \mathrm{C}$ \\
\hline ATP Content, Extracellular & bEnd.3 & 0.5056 & 0.0011 & Fig. 4.4D \\
\hline Mitochondrial Superoxide & bEnd.3 & 0.1897 & 0.0126 & Fig. 4.5D \\
\hline Mitochondrial Superoxide & $\begin{array}{c}\text { Isolated } \\
\text { Mitochondria }\end{array}$ & 0.9637 & $<0.0001$ & Fig. 4.5E \\
\hline
\end{tabular}

Supplemental Table 4.1. Analysis of linear trend (one-way ANOVA) was performed on experimental data to determine if the measurements are dose-dependent. Assessment: measured parameter, Subject: entity receiving treatment, Correlation Coefficient: $\mathrm{R}^{2}$ value estimates the strength that the linear model describes the data, Linear Trend: $p$-value indicates whether there is a significant relationship between the model and the data, Data Depiction: figure number and panel to where the specified data is located. bEnd. 3 (cell line), Primary Endothelial (primary cerebrovascular endothelial cells), isolated mitochondria (mitochondria isolated from primary cerebrovascular endothelial cells). 
It is firmly established that mitochondrial activity is modulated by $\mathrm{Ca}^{2+}$. The influx of $\mathrm{Ca}^{2+}$ to the mitochondrial matrix can exert both stimulatory and inhibitory effects on mitochondrial function. Mitochondrial influx of $\mathrm{Ca}^{2+}$ results in upregulated oxidative phosphorylation by activating mitochondrial dehydrogenase enzymes, glycerophosphate dehydrogenase, isocitrate dehydrogenase, pyruvate dehydrogenase, and oxoglutarate dehydrogenase (Hansford and Chappell 1967; Denton et al. 1972; McCormack and Denton 1979). Activation of these enzymes by $\mathrm{Ca}^{2+}$ increases mitochondrial oxidative phosphorylation and ATP production (McCormack et al. 1990). We found that amyloid- $\beta$ causes increased mitochondrial matrix calcium while decreasing cytosolic calcium in bEnd.3 cells. The stimulatory activity of calcium to resident dehydrogenase enzymes in the mitochondrial matrix may account for the elevated respiration we observed in both bEnd.3 cells and in primary cerebrovascular endothelial cells. In addition, increased concentrations of mitochondrial matrix $\mathrm{Ca}^{2+}$ have been shown to cause the formation of the permeability transition pore (PTP) on the mitochondrial membrane, allowing the release of apoptotic signaling molecules which facilitate mitochondrial-mediated apoptosis (Kroemer and Reed 2000; Deniaud et al. 2008; Orrenius et al. 2003). Thus, the cytotoxicity we observed following prolong exposure to amyloid- $\beta$ may be driven by the PTP following amyloid- $\beta$-induced accumulation of mitochondrial matrix calcium.

Exposure to amyloid- $\beta$ in both bEnd. 3 cells and in primary endothelial cells resulted in increased oxygen consumption for ATP production and intracellular ATP content. This upregulated ATP synthesis may be an effect of the increased calcium levels in the mitochondrial matrix and its stimulatory role with mitochondrial dehydrogenase enzymes (Hansford and Chappell 1967; Denton et al. 1972; McCormack and Denton 1979). The 
elevated activity of mitochondrial dehydrogenase enzyme enhances the production of NADH. Catabolism of NADH to NAD+ increases the proton-motive force by serving as a proton donor substrate, increasing the throughput of hydrogen ions pumped into the mitochondrial intermembrane space at complex I. The increased hydrogen ions pumped across the mitochondrial inner membrane drives the accelerated ATP synthesis by mechanically powering the coupling of ADP to inorganic phosphate by ATP synthase.

Mitochondrial superoxide production is sensitive to the proton-motive force (Brand et al. 2004). High rates of electron transport can cause its reversal, delivering electrons to complex I; these electrons are coupled to molecular oxygen and produce superoxide radicals (Chouchani et al. 2016). Indeed, exposure to amyloid- $\beta$ resulted in an increased production of mitochondrial superoxide in intact cells and in isolated mitochondria. These observations indicate that the aberrant elevated mitochondrial respiration following amyloid- $\beta$ exposure is inefficient, resulting in increased production of reactive oxygen species where it can potentially damage the cell and subsequently lead to cell death.

Since mitochondrial calcium is implicated in many of the cellular changes following amyloid- $\beta$ exposure, we hypothesized that blocking the amyloid- $\beta$-induced mitochondrial calcium accumulation would mitigate the elevated mitochondrial respiration, superoxide production, and cell death following exposure to amyloid- $\beta$. Chelation of extracellular calcium with EDTA was effective at preventing the amyloid- $\beta$-induced accumulation of calcium in the mitochondrial matrix. Thus, we evaluated the role of mitochondrial calcium accumulation as central to the mechanism underlying amyloid- $\beta$ mediated endothelial cell injury. We found that by preventing calcium accumulation in the mitochondria, the elevated respiration following amyloid- $\beta$ could be mitigated. In addition, blocking the amyloid- $\beta$ associated 
ANOVA Summary

\begin{tabular}{|c|c|c|c|c|c|}
\hline Assay & $\begin{array}{c}\text { F- } \\
\text { value }\end{array}$ & P-value & $\mathbf{R}^{2}$ & $F(D F n, D F d)$ & $\begin{array}{c}\text { Data } \\
\text { Depiction } \\
\text { (Figure, Panel) }\end{array}$ \\
\hline Oxygen Consumption & 17.96 & $<0.0001$ & n.a. & 57 & Fig. $4.2 \mathrm{~A}$ \\
\hline Basal Respiration & 59.70 & $<0.0001$ & 0.8028 & 3,44 & Fig. $4.2 \mathrm{~B}$ \\
\hline Maximum Respiration & 91.92 & $<0.0001$ & 0.8733 & 3,44 & Fig. $4.2 \mathrm{C}$ \\
\hline Spare Capacity & 46.50 & $<0.0001$ & 0.7602 & 3,44 & Fig. 4.2D \\
\hline Proton Leak & 56.33 & $<0.0001$ & 0.8086 & 3,44 & Fig. 4.2E \\
\hline Basal Respiration & 12.08 & $<0.0001$ & 0.6016 & 3,24 & Fig. $4.2 \mathrm{~F}$ \\
\hline Maximum Respiration & 24.03 & $<0.0001$ & 0.7503 & 3,24 & Fig. $4.2 \mathrm{G}$ \\
\hline Spare Capacity & 9.77 & 0.0002 & 0.55 & 3,24 & Fig. $4.2 \mathrm{H}$ \\
\hline Proton Leak & 20.88 & $<0.0001$ & 0.723 & 3,24 & Fig. 4.2I \\
\hline Mitochondrial Calcium & 12.58 & $<0.0001$ & 0.4395 & 3,40 & Fig. $4.3 \mathrm{~A}$ \\
\hline Cytosolic Calcium & 35.19 & $<0.0001$ & 0.7457 & 3,36 & Fig. 4.3B \\
\hline ATP Production & 46.92 & $<0.0001$ & 0.7618 & 3,44 & Fig. 4.4A \\
\hline ATP Production & 5.71 & 0.0034 & 0.3715 & 3,29 & Fig. 4.4B \\
\hline ATP Content, Intracellular & 60.05 & $<0.0001$ & 0.8037 & 3,44 & Fig. $4.4 \mathrm{C}$ \\
\hline ATP Content, Extracellular & 9.13 & 0.0008 & 0.6171 & 3,29 & Fig. 4.4D \\
\hline Mitochondrial Superoxide & 23.68 & $<0.0001$ & 0.6764 & 3,44 & Fig. 4.5D \\
\hline Mitochondrial Superoxide, Isolated Mitochondria & 381.20 & $<0.0001$ & 0.9871 & 3,17 & Fig. 4.5E \\
\hline Mitochondrial Calcium & 15.46 & $<0.0001$ & 0.5189 & 3,34 & Fig. 4.6A \\
\hline Basal Respiration & 51.69 & $<0.0001$ & 0.866 & 4,20 & Fig. 4.6B \\
\hline Maximum Respiration & 56.03 & $<0.0001$ & 0.8751 & 3,43 & Fig. 4.6C \\
\hline Spare Capacity & 216.20 & $<0.0001$ & 0.9658 & 3,24 & Fig. 4.6D \\
\hline Proton Leak & 61.23 & $<0.0001$ & 0.8844 & 3,24 & Fig. 4.6E \\
\hline ATP Production & 126.90 & $<0.0001$ & 0.9407 & 3,24 & Fig. 4.6F \\
\hline Mitochondrial Superoxide & 90.11 & $<0.0001$ & 0.8739 & 3,39 & Fig. 4.6G \\
\hline
\end{tabular}


calcium in the mitochondrial matrix, the elevated ATP production and superoxide generation could be halted. These data suggest that calcium influx to mitochondria is a central component to the mechanism mediated by amyloid- $\beta$ during the early phase of cellular injury in cerebrovascular endothelial cells.

We further investigated the route by which calcium influx through the plasma membrane enters mitochondria. Specifically, we assessed the contribution of calcium to the mitochondria through the endoplasmic reticulum $\mathrm{IP}_{3} \mathrm{R}$, reverse mode $\mathrm{mNCX}$, and the PTP. Each pathway was evaluated by exposing cells to $A \beta_{1-42}$ in the presence of specific antagonists then measuring mitochondrial calcium content.

Our data describe a major role for extracellular calcium in the mechanism mediating the cytotoxicity of $A \beta_{1-42}$. We found that chelation of extracellular calcium prevented its accumulation in mitochondria (Figure 4.6) and the increased oxidative phosphorylation and superoxide production by mitochondria. Recent studies have demonstrated that exposure of the cell membrane to $A \beta$ results in increased permeability of the bilayer, caused by the insertion of $\mathrm{A} \beta$ into the membrane, forming ion conductive channels (Bode et al. 2017; Demuro et al. 2005). In principal, these channels would allow the influx of calcium into the cell. Furthermore, we used agents that antagonize mPTP formation, $\mathrm{mNCX}$, and $\mathrm{IP}_{3} \mathrm{R}$ to evaluate the contribution of intracellular calcium regulatory pathways in the accumulation of calcium in mitochondria. In our experiments, inhibition of any of these three calcium pathways resulted in attenuated calcium influx into mitochondria. Therefore, these data suggest that exposure to $A \beta_{1-42}$ causes the cell membrane to become conductive to calcium, the resulting influx of calcium is shunted into mitochondria via multiple calcium regulatory pathways that cause the hyperoxidative state of mitochondria observed in endothelial cell mitochondria. 
It is well known that mitochondria possess a sodium / calcium exchanger ( $\mathrm{mNCX})$ that functions in the efflux of calcium from mitochondria (Hoyt et al. 1998; Kiedrowski 1999). It is possible for mNCX to function in reverse mode during conditions of metabolic and calcium dysregulation (Griffiths 1999; Smets et al. 2004), transporting calcium into the mitochondria. To assess this possibility, we used KB-R7943, an inhibitor of reverse mode mNCX at concentrations previously used in experiments (Brustovetsky et al. 2011), and observed a

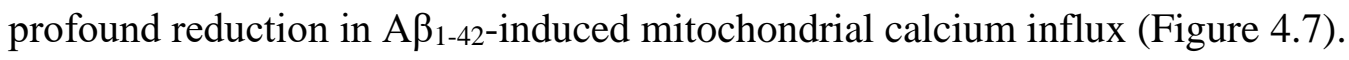

Mitochondrial calcium uptake is a critical cellular function that maintains low cytosolic calcium concentrations. Calcium influx into the mitochondrial matrix also participates in a regulatory role facilitating mitochondrial dehydrogenase enzyme activity. However, excessive accumulation of calcium by mitochondria may lead to mitochondrial damage via the induction of the mPTP. Calcium-dependent induction of the mPTP is a major mechanism of calciuminduced damage to mitochondria.

Calcium uptake into mitochondria primarily occurs via diffusion across the outer mitochondrial membrane (OMM) through the voltage-dependent anion channel (VDAC). Translocation of hydrogen by the respiratory chain forms the mitochondrial membrane potential that serves as the driving force to transport calcium across the inner mitochondrial membrane (IMM), down its electrochemical gradient. Calcium influx into the mitochondrial matrix is mediated by the mitochondrial calcium uniporter (MCU) at the inner mitochondrial membrane. The MCU channel conducts calcium ions from the outer membrane space into the mitochondrial matrix. Antagonism of MCU substantially reduced $\mathrm{A} \beta_{1-42 \text {-induced }}$ mitochondrial calcium influx (Figure 4.7). 
The efflux of calcium from the endoplasmic reticulum via $\mathrm{IP}_{3} \mathrm{R}$ produce localized microdomains of elevated calcium concentration that are crucial for calcium uptake by the mitochondria (Rizzuto et al. 1998). The contact sites that hold mitochondria stationary and within the calcium-rich microdomain occurs at regions termed mitochondrial associated membranes (MAMs). MAMs are sites involved in the exchange of biomolecules, such as lipids, reactive oxygen species, and calcium between the endoplasmic reticulum and mitochondria. At MAM sites, calcium is conducted into the mitochondrial intermembrane space via a large protein complex involving VDAC. To determine whether the influx of calcium into mitochondria following $A \beta_{1-42}$ exposure involves the $\mathrm{IP}_{3} \mathrm{R}$ pathway, we used 2$\mathrm{APB}$, an $\mathrm{IP}_{3} \mathrm{R}$ antagonist. Inhibition of $\mathrm{IP}_{3} \mathrm{R}$ eliminated $\mathrm{A} \beta_{1-42 \text {-induced mitochondrial calcium }}$ influx (Figure 4.7).

Together, these data describe a role for calcium in the cytotoxicity exerted by $A \beta_{1-42}$, where the cell membrane becomes conductive to extracellular calcium, therefrom the influx of calcium is shunted into the mitochondria via multiple calcium regulatory pathways, causing the hyperoxidative state of mitochondria observed following exposure to $A \beta_{1-42}$.

Consistent with the literature, we observed morphological changes to the mitochondrial network, characterized by punctated mitochondria via excessive fission (Wang et al. 2008; Bartolome et al. 2018; Zhang et al. 2016; Almeida and Medina 1998; Cho et al. 2009). Mitochondrial fission and fusion is a dynamic process modulated by the energy demand of the cell and participates in the quality control of the mitochondrial network. Damage to mitochondria, such as from reactive oxygen species can cause mitochondrial fragmentation, similar to our observation in vascular endothelial cells (Ježek et al. 2018; Wu et al. 2011; Willems et al. 2015; Hung et al. 2018). Thus, we interpret these data to suggest that the 
fragmentation of mitochondria in bEnd.3 cells is mediated by the elevated mitochondrial superoxide production following amyloid- $\beta$ exposure. Therefore, because we found that the amyloid- $\beta$ associated elevation of mitochondrial superoxide production could be mitigated by preventing mitochondrial matrix calcium. As a result, we expect that the fragmentation of mitochondria could be abrogated. Indeed, we found that by reducing mitochondrial calcium via chelation halted the aberrant mitochondrial fission following amyloid- $\beta$ exposure, lending evidence to a mechanism involving aberrant mitochondrial fission driven by the oxidative damage to mitochondria in cerebrovascular endothelial cells exposed to amyloid- $\beta$. Lastly, when we prevented the amyloid- $\beta$-induced accumulation of mitochondrial matrix calcium, the apoptotic morphology of endothelial cells was completely prevented.

Overall, our study provides evidence of early events of cellular injury that involves the elevation of mitochondrial respiration, calcium accumulation in mitochondria, and the production of superoxide. We found that calcium influx into the mitochondria is a central component to the mechanism mediating cell injury. Furthermore, our data indicates that blocking mitochondrial calcium can completely halt the pathological mechanism mediating cell death. In addition, these findings provide a mechanism for the mitochondrial dysfunction and deficits observed in $\mathrm{AD}$ and provide evidence for a therapeutic strategy that targets mitochondrial matrix calcium.

\section{Funding Acknowledgements}

This work was supported by the NIH grants P20 GM109098, P01 AG027956, U54 GM104942, T32 AG052375, K01 NS081014, and K01 MH117343. Imaging experiments and image 
analyses were performed in the West Virginia University Microscope Imaging Facility, which has been supported by the Mary Babb Randolph Cancer Center and NIH grants P20 RR016440, P30 RR032138/GM103488 and P20 RR016477.

\section{Declaration of Conflicting Interests}

The author(s) declare(s) that there is no conflict of interest.

\section{Supplemental Information Statement}

Supplemental material for this article can be found at the journal website:

\section{References}

Ainscow E. K., Brand M. D. (1999) Top-down control analysis of ATP turnover, glycolysis and oxidative phosphorylation in rat hepatocytes. Eur. J. Biochem. 263, 671-85.

Almeida A., Medina J. M. (1998) A rapid method for the isolation of metabolically active mitochondria from rat neurons and astrocytes in primary culture. Brain Res. Protoc. 2, 209-214.

Bartolome F., la Cueva M. de, Pascual C., Antequera D., Fernandez T., Gil C., Martinez A., Carro E. (2018) Amyloid $\beta$-induced impairments on mitochondrial dynamics, hippocampal neurogenesis, and memory are restored by phosphodiesterase 7 inhibition. Alzheimers. Res. Ther. 10, 24.

Bastianetto S., Ramassamy C., Doré S., Christen Y., Poirier J., Quirion R. (2000) The Ginkgo biloba extract (EGb 761) protects hippocampal neurons against cell death induced by beta-amyloid. Eur. J. Neurosci. 12, 1882-90.

Behl C., Davis J. B., Lesley R., Schubert D. (1994) Hydrogen peroxide mediates amyloid $\beta$ protein toxicity. Cell 77, 817-827. 
Bode D. C., Baker M. D., Viles J. H. (2017) Ion channel formation by amyloid- $\beta 42$ oligomers but not amyloid- $\beta 40$ in cellular membranes. J. Biol. Chem. 292, 144-1413.

Brace L. E., Vose S. C., Stanya K., Gathungu R. M., Marur V. R., Longchamp A., TreviñoVillarreal H., et al. (2016) Increased oxidative phosphorylation in response to acute and chronic DNA damage. NPJ aging Mech. Dis. 2, 16022.

Brand M. D., Affourtit C., Esteves T. C., Green K., Lambert A. J., Miwa S., Pakay J. L., Parker N. (2004) Mitochondrial superoxide: production, biological effects, and activation of uncoupling proteins. Free Radic. Biol. Med. 37, 755-767.

Brown G. C., Lakin-Thomas P. L., Brand M. D. (1990) Control of respiration and oxidative phosphorylation in isolated rat liver cells. Eur. J. Biochem. 192, 355-62.

Brundel M., Bresser J. de, Dillen J. J. van, Kappelle L. J., Biessels G. J. (2012a) Cerebral microinfarcts: a systematic review of neuropathological studies. J. Cereb. Blood Flow Metab. 32, 425-436.

Brundel M., Heringa S. M., Bresser J. de, Koek H. L., Zwanenburg J. J. M., Jaap Kappelle L., Luijten P. R., Biessels G. J. (2012b) High prevalence of cerebral microbleeds at 7Tesla MRI in patients with early Alzheimer's disease. J. Alzheimers. Dis. 31, 259-263.

Brustovetsky T., Brittain M. K., Sheets P. L., Cummins T. R., Pinelis V., Brustovetsky N. (2011) KB-R7943, an inhibitor of the reverse $\mathrm{Na}+/ \mathrm{Ca} 2+$ exchanger, blocks N-methylD-aspartate receptor and inhibits mitochondrial complex i. Br. J. Pharmacol. 162, 255270.

Castellani R. J., Rolston R. K., Smith M. A. (2010) Alzheimer disease. Dis. Mon. 56, 484-546.

Chi N.-F., Chien L.-N., Ku H.-L., Hu C.-J., Chiou H.-Y. (2013) Alzheimer disease and risk of stroke: a population-based cohort study. Neurology 80, 705-711.

Cho D.-H., Nakamura T., Fang J., Cieplak P., Godzik A., Gu Z., Lipton S. A. (2009) Snitrosylation of Drp1 mediates beta-amyloid-related mitochondrial fission and neuronal injury. Science 324, 102-5.

Choi S. W., Gerencser A. A., Nicholls D. G. (2009) Bioenergetic analysis of isolated cerebrocortical nerve terminals on a microgram scale: spare respiratory capacity and 
stochastic mitochondrial failure. J. Neurochem. 109, 1179-91.

Chouchani E. T., Pell V. R., James A. M., Work L. M., Saeb-Parsy K., Frezza C., Krieg T., Murphy M. P. (2016) A Unifying Mechanism for Mitochondrial Superoxide Production during Ischemia-Reperfusion Injury. Cell Metab. 23, 254-263.

Demuro A., Mina E., Kayed R., Milton S. C., Parker I., Glabe C. G. (2005) Calcium dysregulation and membrane disruption as a ubiquitous neurotoxic mechanism of soluble amyloid oligomers. J. Biol. Chem. 280, 17294-300.

Deniaud A., Sharaf el dein O., Maillier E., Poncet D., Kroemer G., Lemaire C., Brenner C. (2008) Endoplasmic reticulum stress induces calcium-dependent permeability transition, mitochondrial outer membrane permeabilization and apoptosis. Oncogene 27, 285-299.

Denton R. M., Randle P. J., Martin B. R. (1972) Stimulation by calcium ions of pyruvate dehydrogenase phosphate phosphatase. Biochem. J. 128, 161-3.

DeSimone C. V., Graff-Radford J., El-Harasis M. A., Rabinstein A. A., Asirvatham S. J., Holmes D. R. (2017) Cerebral Amyloid Angiopathy: Diagnosis, Clinical Implications, and Management Strategies in Atrial Fibrillation. J. Am. Coll. Cardiol. 70, 1173-1182.

Dias Zeidler J., Fernandes-Siqueira L. O., Carvalho A. S., Cararo-Lopes E., Dias M. H. S., Ketzer L. A., Galina A., Poian A. T. Da (2017) Mitochondrial oxidation of specific substrates Short-term starvation is a strategy to unravel the cellular capacity of oxidizing specific exogenous/endogenous substrates in mitochondria.

Downs C. A., Heckathorn S. A. (1998) The mitochondrial small heat-shock protein protects NADH:ubiquinone oxidoreductase of the electron transport chain during heat stress in plants. FEBS Lett. 430, 246-250.

Drummond G. R., Selemidis S., Griendling K. K., Sobey C. G. (2011) Combating oxidative stress in vascular disease: NADPH oxidases as therapeutic targets. Nat. Rev. Drug Discov. 10, 453-471.

Du H., Guo L., Yan S., Sosunov A. A., McKhann G. M., Yan S. S. (2010) Early deficits in synaptic mitochondria in an Alzheimer's disease mouse model. Proc. Natl. Acad. Sci. U. S. A. 107, 18670-5. 
DuBoff B., Feany M., Götz J. (2013) Why size matters - balancing mitochondrial dynamics in Alzheimer's disease. Trends Neurosci. 36, 325-335.

Ferreira A., Sinjoanu R. C., Nicholson A., Kleinschmidt S. (2010) A $\beta$ Toxicity in Primary Cultured Neurons, in Methods Mol. Biol., Vol. 670, pp. 141-153.

Garlid K. D., Paucek P. (2003) Mitochondrial potassium transport: the K+ cycle. Biochim. Biophys. Acta - Bioenerg. 1606, 23-41.

Gillardon F., Rist W., Kussmaul L., Vogel J., Berg M., Danzer K., Kraut N., Hengerer B. (2007) Proteomic and functional alterations in brain mitochondria from Tg2576 mice occur before amyloid plaque deposition. Proteomics 7, 605-616.

Griffiths E. J. (1999) Reversal of mitochondrial Na/Ca exchange during metabolic inhibition in rat cardiomyocytes. FEBS Lett. 453, 400-4.

Han B. H., Zhou M.-L., Johnson A. W., Singh I., Liao F., Vellimana A. K., Nelson J. W., et al. (2015) Contribution of reactive oxygen species to cerebral amyloid angiopathy, vasomotor dysfunction, and microhemorrhage in aged Tg2576 mice. Proc. Natl. Acad. Sci. U. S. A. 112, E881-90.

Hansford R. G., Chappell J. B. (1967) The effect of Ca2+ on the oxidation of glycerol phosphate by blowfly flight-muscle mitochondria. Biochem. Biophys. Res. Commun. 27, 686-92.

Hirai K., Aliev G., Nunomura A., Fujioka H., Russell R. L., Atwood C. S., Johnson A. B., et al. (2001) Mitochondrial abnormalities in Alzheimer's disease. J. Neurosci. 21, 3017-23.

Hoyt K. R., Arden S. R., Aizenman E., Reynolds I. J. (1998) Reverse Na+/Ca2+ exchange contributes to glutamate-induced intracellular $\mathrm{Ca} 2+$ concentration increases in cultured rat forebrain neurons. Mol. Pharmacol. 53, 742-9.

Hung C. H.-L., Cheng S. S.-Y., Cheung Y.-T., Wuwongse S., Zhang N. Q., Ho Y.-S., Lee S. M.-Y., Chang R. C.-C. (2018) A reciprocal relationship between reactive oxygen species and mitochondrial dynamics in neurodegeneration. Redox Biol. 14, 7-19.

Janson C. G. (2015) AD and CAA: Independent risk factors for dementia. Sci. Transl. Med. 7, $318 \mathrm{ec} 214-318 \mathrm{ec} 214$. 
Jarmuszkiewicz W., Woyda-Ploszczyca A., Koziel A., Majerczak J., Zoladz J. A. (2015) Temperature controls oxidative phosphorylation and reactive oxygen species production through uncoupling in rat skeletal muscle mitochondria. Free Radic. Biol. Med. 83, $12-$ 20.

Jellinger K. A. (2010) Prevalence and impact of cerebrovascular lesions in Alzheimer and lewy body diseases. Neurodegener. Dis. 7, 112-115.

Ježek J., Cooper K. F., Strich R. (2018) Reactive Oxygen Species and Mitochondrial Dynamics: The Yin and Yang of Mitochondrial Dysfunction and Cancer Progression. Antioxidants (Basel, Switzerland) 7.

Kalaria R. N. (2010) Vascular basis for brain degeneration: faltering controls and risk factors for dementia. Nutr. Rev. 68 Suppl 2, S74-87.

Kiedrowski L. (1999) N-methyl-D-aspartate excitotoxicity: Relationships among plasma membrane potential, $\mathrm{Na}+\mathrm{Ca} 2+$ exchange, mitochondrial $\mathrm{Ca} 2+$ overload, and cytoplasmic concentrations of $\mathrm{Ca} 2+\mathrm{H}+$ and $\mathrm{K}+$. Mol. Pharmacol. 56, 619-632.

Kroemer G., Reed J. C. (2000) Mitochondrial control of cell death. Nat. Med. 6, 513-519.

la Torre J. C. de (2004) Alzheimer's disease is a vasocognopathy: a new term to describe its nature. Neurol. Res. 26, 517-524.

la Torre J. C. de (2010) Vascular risk factor detection and control may prevent Alzheimer's disease. Ageing Res. Rev. 9, 218-225.

LaFerla F. M. (2002) Calcium dyshomeostasis and intracellular signalling in alzheimer's disease. Nat. Rev. Neurosci. 3, 862-872.

Manczak M., Calkins M. J., Reddy P. H. (2011) Impaired mitochondrial dynamics and abnormal interaction of amyloid beta with mitochondrial protein Drp1 in neurons from patients with Alzheimer's disease: implications for neuronal damage. Hum. Mol. Genet. 20, 2495-2509.

McCormack J. G., Denton R. M. (1979) The effects of calcium ions and adenine nucleotides on the activity of pig heart 2-oxoglutarate dehydrogenase complex. Biochem. J. 180, 53344. 
McCormack J. G., Halestrap A. P., Denton R. M. (1990) Role of calcium ions in regulation of mammalian intramitochondrial metabolism. Physiol. Rev. 70, 391-425.

Miller A. A., Drummond G. R., Schmidt H. H. H. W., Sobey C. G. (2005) NADPH Oxidase Activity and Function Are Profoundly Greater in Cerebral Versus Systemic Arteries. Circ. Res. 97, 1055-1062.

Mookerjee S. A., Gerencser A. A., Nicholls D. G., Brand M. D. (2017) Quantifying intracellular rates of glycolytic and oxidative ATP production and consumption using extracellular flux measurements. J. Biol. Chem. 292, 7189-7207.

Orrenius S., Zhivotovsky B., Nicotera P. (2003) Regulation of cell death: the calciumapoptosis link. Nat. Rev. Mol. Cell Biol. 4, 552-565.

Park L., Anrather J., Forster C., Kazama K., Carlson G. A., Iadecola C. (2004) A $\beta$-Induced Vascular Oxidative Stress and Attenuation of Functional Hyperemia in Mouse Somatosensory Cortex. J. Cereb. Blood Flow Metab. 24, 334-342.

Park L., Anrather J., Girouard H., Zhou P., Iadecola C. (2007) Nox2-Derived Reactive Oxygen Species Mediate Neurovascular Dysregulation in the Aging Mouse Brain. J. Cereb. Blood Flow Metab. 27, 1908-1918.

Park L., Anrather J., Zhou P., Frys K., Pitstick R., Younkin S., Carlson G. A., Iadecola C. (2005) NADPH-oxidase-derived reactive oxygen species mediate the cerebrovascular dysfunction induced by the amyloid beta peptide. J. Neurosci. 25, 1769-77.

Pilchova I., Klacanova K., Tatarkova Z., Kaplan P., Racay P. (2017) The Involvement of Mg ${ }^{2+}$ in Regulation of Cellular and Mitochondrial Functions. Oxid. Med. Cell. Longev. 2017, $1-8$.

Purandare N., Burns A. (2009) Cerebral emboli in the genesis of dementia. J. Neurol. Sci. 283, $17-20$.

Qi X., Disatnik M.-H., Shen N., Sobel R. A., Mochly-Rosen D. (2011) Aberrant mitochondrial fission in neurons induced by protein kinase $\mathrm{C} \delta$ under oxidative stress conditions in vivo. Mol. Biol. Cell 22, 256-265.

Querfurth H. W., LaFerla F. M. (2010) Alzheimer's Disease. N. Engl. J. Med. 362, 329-344. 
Quintana D. D., Garcia J. A., Sarkar S. N., Jun S., Engler-Chiurazzi E. B., Russell A. E., Cavendish J. Z., Simpkins J. W. (2018) Hypoxia-reoxygenation of primary astrocytes results in a redistribution of mitochondrial size and mitophagy. Mitochondrion.

Rizzuto R., Pinton P., Carrington W., Fay F. S., Fogarty K. E., Lifshitz L. M., Tuft R. A., Pozzan T. (1998) Close contacts with the endoplasmic reticulum as determinants of mitochondrial Ca2+ responses. Science (80-. ). 280, 1763-1766.

Smets I., Caplanusi A., Despa S., Molnar Z., Radu M., VandeVen M., Ameloot M., Steels P. (2004) $\mathrm{Ca} 2+$ uptake in mitochondria occurs via the reverse action of the $\mathrm{Na}+\mathrm{Ca} 2+$ exchanger in metabolically inhibited MDCK cells. Am. J. Physiol. - Ren. Physiol. 286, F784-94.

Takuma H., Tomiyama T., Kuida K., Mori H. (2004) Amyloid Beta Peptide-Induced Cerebral Neuronal Loss Is Mediated By Caspase-3 In Vivo. J. Neuropathol. Exp. Neurol. 63, 255261.

Tolppanen A.-M., Lavikainen P., Solomon A., Kivipelto M., Soininen H., Hartikainen S. (2013) Incidence of stroke in people with Alzheimer disease: a national register-based approach. Neurology 80, 353-358.

Trimmer P. A., Swerdlow R. H., Parks J. K., Keeney P., Bennett J. P., Miller S. W., Davis R. E., Parker W. D. (2000) Abnormal Mitochondrial Morphology in Sporadic Parkinson's and Alzheimer's Disease Cybrid Cell Lines. Exp. Neurol. 162, 37-50.

Vinters H. V (1987) Cerebral amyloid angiopathy. A critical review. Stroke 18, 311-324.

Wang W., Yin J., Ma X., Zhao F., Siedlak S. L., Wang Z., Torres S., et al. (2017) Inhibition of mitochondrial fragmentation protects against Alzheimer's disease in rodent model. Hum. Mol. Genet. 26, 4118-4131.

Wang X., Su B., Siedlak S. L., Moreira P. I., Fujioka H., Wang Y., Casadesus G., Zhu X. (2008) Amyloid-beta overproduction causes abnormal mitochondrial dynamics via differential modulation of mitochondrial fission/fusion proteins. Proc. Natl. Acad. Sci. U. S. A. 105, 19318-23.

Willems P. H. G. M., Rossignol R., Dieteren C. E. J., Murphy M. P., Koopman W. J. H. (2015) Cell Metabolism Perspective Redox Homeostasis and Mitochondrial Dynamics. Cell 
Metab. 22, 207-218.

Wu S., Zhou F., Zhang Z., Xing D. (2011) Mitochondrial oxidative stress causes mitochondrial fragmentation via differential modulation of mitochondrial fission-fusion proteins. FEBS J. 278, 941-954.

Xie H., Guan J., Borrelli L. A., Xu J., Serrano-Pozo A., Bacskai B. J. (2013) Mitochondrial alterations near amyloid plaques in an Alzheimer's disease mouse model. J. Neurosci. 33, $17042-51$.

Yadava N., Nicholls D. G. (2007) Spare respiratory capacity rather than oxidative stress regulates glutamate excitotoxicity after partial respiratory inhibition of mitochondrial complex I with rotenone. J. Neurosci. 27, 7310-7.

Yajima D., Motani H., Hayakawa M., Sato Y., Sato K., Iwase H. (2009) The relationship between cell membrane damage and lipid peroxidation under the condition of hypoxiareoxygenation: analysis of the mechanism using antioxidants and electron transport inhibitors. Cell Biochem. Funct. 27, 338-343.

Yao J., Irwin R. W., Zhao L., Nilsen J., Hamilton R. T., Brinton R. D. (2009) Mitochondrial bioenergetic deficit precedes Alzheimer's pathology in female mouse model of Alzheimer's disease. Proc. Natl. Acad. Sci. 106, 14670-14675.

Youle R. J., Bliek A. M. van der (2012) Mitochondrial fission, fusion, and stress. Science 337, $1062-5$.

Youle R. J., Narendra D. P. (2011) Mechanisms of mitophagy. Nat. Rev. Mol. Cell Biol. 12, $9-14$.

Zhang L., Trushin S., Christensen T. A., Bachmeier B. V., Gateno B., Schroeder A., Yao J., et al. (2016) Altered brain energetics induces mitochondrial fission arrest in Alzheimer's Disease. Sci. Rep. 6, 18725. 


\section{CHAPTER 5}

\section{The Cerebral Angiome: High Resolution MicroCT Imaging of the Whole Brain Cerebrovasculature in Female and Male Mice}

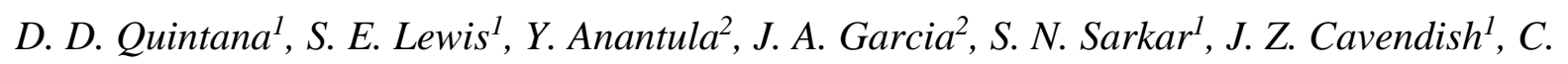
M. Brown ${ }^{2}$ and J. W. Simpkins ${ }^{1 *}$

${ }^{1}$ Department of Physiology and Pharmacology

${ }^{2}$ Department of Neuroscience

Center for Basic Translational and Stroke Research

West Virginia University, Morgantown, West Virginia, 26506

\section{Author Contribution Statement}

DDQ designed studies, conducted studies and composed the manuscript. SEL, YA, JAG and JZC conducted studies. SNS and CMB revised the manuscript. JWS designed studies and revised the manuscript. 


\begin{abstract}
The cerebrovascular system provides crucial functions that maintain metabolic and homeostatic states of the brain. Despite its integral role of supporting cerebral viability, the topological organization of these networks remains largely uncharacterized. This void in our knowledge surmises entirely from current technological limitations that prevent the capturing of data through the entire depth of the brain. We report high-resolution reconstruction and analysis of the complete vascular network of the entire brain at the capillary level in adult female and male mice using a vascular corrosion cast procedure. Vascular network analysis of the whole brain revealed sex-related differences of vessel hierarchy. In addition, regionspecific network analysis demonstrated different patterns of angioarchitecture between brain subregions and sex. Furthermore, our group is the first to provide a three-dimensional analysis of the angioarchitecture and network organization in a single reconstructed tomographic data set that encompasses all hierarchy of vessels in the brain of the adult mouse.
\end{abstract}




\section{Introduction}

The cerebral capillary network is the interface between the brain and the periphery and is therefore decisive for adequate delivery of oxygen and nutrients to the brain. Understanding capillary network topology and the angioarchitecture of the conduit vessels that move blood through capillaries is of paramount importance to understand the metabolic activity and function of the nervous system. Despite its unique positioning as the nexus for advancement in a number of fields, a complete description of the topological basis of the cerebral angioarchitecture has not been obtained.

The microvasculature, consisting of the smallest capillaries, has proven hard to image (Demené et al., 2016; Starosolski et al., 2015). Traditional imaging methods such as ultrasound and magnetic resonance angiography do not have a resolution that is high enough to detect the microvasculature (Fukuda, Moon, Wang, \& Kim, 2006; Harel, Lin, Moeller, Ugurbil, \& Yacoub, 2006; Uğurbil et al., 2003). Confocal single-photon and two-photon imaging can provide the resolution needed but have depth limitations (P. S. Tsai et al., 2009). To resolve the resolution and depth problems, micro-CT imaging, with contrast, can be used. Contrast agents that fill the vessels have made it possible to capture the vasculature in whole organs in 3-D and the same is true for the brain (Beckmann et al., 2003; Heinzer et al., 2006; Krucker, Schuler, Meyer, Staufenbiel, \& Beckmann, 2004; Meyer, Ulmann-Schuler, Staufenbiel, \& Krucker, 2008).

Light sheet microscopy and laser sheet microscopy (LSM) has become a popular platform for imaging of large biological specimens. However, because LSM is an optical imaging method, the quality of the images obtained is dependent on the fluorescence and transparency of the specimen and similar to other fluorescence based imaging platforms, is 
prone to image aberrations, blurring, scaling by refractive index, autofluorescence, and signal degradation that progressively get worst with acquisition time and imaging depth. Although despite the disadvantages to LSM, we must consider the advantages to its use. These advantages include superior spatiotemporal resolution, excellent optical sectioning, and its low phototoxicity to live specimens. Furthermore, a well-performed study by Kennel (2018) has demonstrated the ability to image the microvascular networks of large tissue volumes using multiview light-sheet fluorescence microscopy (LSFM), enabling highly accurate reconstruction even when images were acquired at relatively low magnification (Kennel, Teyssedre, Colombelli, \& Plouraboué, 2018).

A growing concern to all fields of research is the ever-growing size of the average data set. This is especially true for data produced by imaging platforms. Our technological achievements made on imaging have been focused on developments that increase the ability to image deeper into specimens and at greater magnification which to produce higher resolution images. Although undeniably beneficial to research, we now face a bottleneck obstructing progress. As the ability to image at a greater volume increases along with progressing resolution, the number of images and size of each image substantially increases, producing large data sets. Large data sets are difficult to work with. Storage and moving data become more of a concern as large data begins to accumulate. Post-processing may need to be performed on computers with computationally powerful capabilities and even more so to perform three-dimensional quantitation and other complex algorithms for vascular network mapping and predictive simulations. We provide a method for imaging the entire brain vasculature, producing a single data set gigabytes in size. 
In the present study, we develop three-dimensional data sets of the cerebrovascular network of the entire brain from nine mice and evaluate the angioarchitecture at the macroand microscopic level in female and male mice. The present research describes an angiome of the cerebrovasculature of the entire brain. The overarching goals of the following analyses were to characterize the geometry, topology, and complexity of the cerebrovasculature of the entire brain, then to focus on region specific angioarchitecture in the primary somatosensory cortex, and to compare cerebrovascular topology between sexes. Each of these overarching goals are divided into three phases: the first phase provides global metrics of the angioarchitecture, the second phase characterizes the angioarchitecture by mapping network topology and generating morphometric descriptions of deconstructed network components, and the final phase focuses on network connectivity and the covariance of morphometry and frequency within the population of network components.

We provide an analysis of the angioarchitecture and network organization in a single volumetric data set encompassing all hierarchy of vessels in the brain of the adult female and male mouse. Our study provides fundamental insight into the cerebral angioarchitecture of female and male mice and describes sexually dimorphic organization that may be of broad interest to the scientific community.

\section{Materials and Methods}

\section{Animal Usage}

Three-month old female $(n=5)$ and male $(n=4)$ C57BL/6J mice were used for whole brain vascular analysis. Power analysis for ANOVA designs indicated a sample size of 4 mice 
per group (power $=0.999)$ for an effect size of $\Delta=1.25$. Mice were housed in accordance to IACUC guidelines of West Virginia University. Animals were maintained under a light/dark cycle (12 h: $12 \mathrm{~h}$ ) with food and water available ad libitum. For all procedures performed in this study that involved the use of laboratory animals were carried out in accordance with the National Institutes of Health guide for the care and use of laboratory animals (NIH Publication No. 8023, revised 1978) and in compliance with the ARRIVE guidelines.

\section{Vascular Corrosion Cast Preparation}

The procedure described below for the preparation of cerebrovascular corrosion casts is shown in Supplemental Figure 5.1. Before beginning the cerebrovascular casting procedure, mice were transported to the surgical suite approximately 4 hours prior to the procedure to allow an acquisition period to a new environment. The acquisition period reduces the amount of stress the animal experiences and prevents any stress-induced changes to the cerebrovasculature. Mice were deeply anesthetized with $4 \%$ isoflurane diffused into a $70 \%$ nitrogen and 30\% oxygen mixture. After confirming deep anesthetization via tail pinch, mice received an intraperitoneal injection of $25 \mathrm{U}$ of heparin in $250 \mu \mathrm{l}$ saline intravenous solution. Mice were then transcardially perfused at $160 \mathrm{mmHg}$ with $0.01 \mathrm{M}$ PBS containing $25 \mathrm{U} / \mathrm{mL}$ of heparin at physiological $\mathrm{pH}$ and warmed to $37^{\circ} \mathrm{C}$. Once blood had been completely removed, mice were perfused at $160 \mathrm{mmHg}$ with $4 \%$ paraformaldehyde warmed to $37^{\circ} \mathrm{C}$. Approximately 5 minutes before complete paraformaldehyde perfusion; the vascular corrosion cast solution was prepared. The PU4ii (VasQtec) corrosion cast solution was prepared by adding $3 \mathrm{~g}(\boldsymbol{\ell})$ of methyl ethyl ketone with 5-10 $\mathrm{mg}$ of blue pigment then mixed thoroughly by vortex. Once the pigment was completely dispersed into the solution, $5 \mathrm{~g}(\boldsymbol{\ell})$ of polyurethane 
resin was added to the solution followed by thorough mixing. Finally, $0.8 \mathrm{~g}(\boldsymbol{\ell})$ of hardener was added to the solution and gently mixed by inverting. After mixing, the final solution was placed into a vacuum chamber for 2 minutes to remove gasses within the solution. The solution was then perfused into the mice at $160 \mathrm{mmHg}$. Once the casting solution began to harden $(3-5$ minutes) and ceased to perfuse the mouse, mice were allowed to remain at room temperature for 4 hours to complete the hardening of the cast. After complete hardening of the cast, mice were decapitated and the skin was removed from the skull with dissecting scissors.

\section{Vascular Corrosion Cast Processing}

The isolated skull was decalcified by immersion in $20 \mathrm{~mL}$ of $8 \%$ formic acid diluted in Milli-Q water then placed in a water bath warmed to $37^{\circ} \mathrm{C}$ for 5 hours. From our experience, it is important to prevent over decalcification of the skull so that the bone tissue becomes flexible without becoming gelatinous and difficult to remove. Once decalcification was complete, the skull was rinsed with distilled water and then immersed in $20 \mathrm{~mL}$ of $8 \%$ potassium hydroxide diluted in Milli-Q water then placed in a water bath warmed to $37^{\circ} \mathrm{C}$ for 4 hours. Following the 4-hour incubation in potassium hydroxide, the skull was rinsed in distilled water and prepared for brain extraction. At this point, the skull was flexible and easy to tare with small forceps. A small incision at the base of the magnum foramen was gently made, and with a pair of small forceps, the incision flap was grasped and pulled upward, breaking the skull up the sagittal suture. With small iris scissors, all major vessels were cut at the floor of the skull by gently lifting the brain. The brain was then removed and the tissue was macerated by immersion into $20 \mathrm{~mL}$ of $8 \%$ potassium hydroxide solution diluted in Milli-Q water then placed in a water bath at $37^{\circ} \mathrm{C}$ overnight. After the overnight incubation, tissue 
maceration is usually incomplete and requires the potassium hydroxide to be replaced and incubated at $37^{\circ} \mathrm{C}$ in a water bath for $24 \mathrm{hrs}$. Once tissue maceration was completed, residual tissue was removed from the vascular cast by washing 3 times for 1 hour with distilled water containing $0.25 \%$ Triton X-100 then rinsed 3 times for 5 minutes with Milli-Q water to remove any residual detergent on the cast.

The cleaned casts were placed into a small container and submerged in $20 \mathrm{~mL}$ of MilliQ water to be frozen for lyophilization. Lyophilization of the casts was performed on a benchtop freeze dry system (Labconco) operating at $-54{ }^{\circ} \mathrm{C}$ and a vacuum pressure of 0.0025 mBar. During the lyophilization process, it is critical that sublimation occurs and that the melting of ice to a liquid state does not occur. The proper sublimation of ice is critical to preserve the physiological architecture of the cerebral blood vessels. In our experience, improper sublimation compromised the structure of the formed vascular casts, often times producing a relatively flattened appearance. Melting of the ice deformed the small capillary structures and was irreversible once the cast completely dried. Successful lyophilization resulted in casts that were rigid and able to maintain moderate pliancy. To allow x-ray detection of the vascular corrosion casts, $6 \mathrm{~mL}$ of a $2 \%$ solution of osmium tetroxide diluted in Milli-Q water was embedded onto the polyurethane casts by immersion and allowed to incubate overnight at $4^{\circ} \mathrm{C}$. Extensive care must be taken when handling solutions or casts containing osmium tetroxide due to its acute toxicity. Proper personal protective equipment should be used and the disposal of waste containing osmium tetroxide should be handled as a p-chemical. Vascular corrosion casts were then removed from the osmium solution with small forceps by gently grasping the cast by the hindbrain. Casts were allowed to air dry for 1 hour, and then mounted with cyanoacrylate adhesive on hexagonal pedestals cut from Plexiglas. 


\section{Technical Notes and Quality Control for Cerebrovascular Corrosion Casts}

Six mice ( 2 male and 4 female) at 3-months of age were used to optimize the perfusion procedure. Optimization of the imaging parameters were performed by selecting one cerebrovascular cast and imaging it 5 separate times, each with adjusted exposure settings, step size, and image averaging. Expectedly, perfusion parameters proved to be most influential to the overall quality of the cast. In our experience, the use of dialysis pumps were most detrimental to the outcome of the cast that seemed to result in "patchy" perfusion of the resin that was observable over the cortex. More so, the use of dialysis pumps usually caused vessel ruptures. We interpret these observations to suggest that the dialysis pump exerts an incompatible perfusion pressure at relatively low flow rate due to the viscosity of the casting resin and its characteristic resistance to flow through the vasculature. To overcome this issue, we found that using a pressurized pump at $160 \mathrm{mmHg}$ produced consistent high-quality casts (Supplemental Figure 5.1B). Noteworthy, we noticed that "good" casts could be predicted and easily observed by the perfusion of several peripheral tissues. First and most simple to observe is the dermis over the premaxilla. A good perfusion resulted in an intense coloration of the dermis to the specific pigment used in the resin composition. A "good" quality cast will often produce a homogenous hue of the skeletal muscles throughout the entire mouse body. However, we found that the perfusion of the liver is most accurately representative of the overall quality of the cerebrovascular cast. This is likely due to the unique physiology of the hepatic vasculature and its sensitivity to systemic perfusion pressure, where it required venous perfusion pressure to drive blood through hepatic circulation then communicate it to the inferior vena cava. Since the hepatic venous system is more extensive than its arterial system, 
the hepatic tissue will not develop a hue specific to the resin pigment if insufficient systemic perfusion pressure occurs. Additionally, if an excess of perfusion pressure is reached, the liver noticeably swells and at a certain threshold will cause hepatic vessels to rupture that can be seen by non-aided observation. Complete perfusion of the cerebrovasculature by the polyurethane resin was visually apparent in that the casting material entered the venous sinus system by traversing through the arterial network (Supplemental Figure 5.1C).

Several days are required for the complete hardening of the cast resin. Removal of the casted brain from the skull is a crucial procedure that requires greater care to prevent damage to the casted vasculature or compromise of its native angioarchitecture. Decalcification of the skull with $8 \%$ formic acid aids in the opening of the skull followed by $8 \%$ potassium hydroxide to destabilize the tough extracellular matrix of the skull and meninges (Supplemental Figure 5.1D). Adequate decalcification and maceration of the skull and meninges is decisive of the recovery of the superior sagittal sinus, transverse sinuses, and inferior cerebral veins. These sinus structures are attached to the meningeal membrane and can easily be broken from the cerebrovasculature when removing the skull. Incidentally, perfusion of the delicate sinus structures is indicative of a complete perfusion of the cerebrovasculature. Specifically, resin that enters the sinus supply must be communicated through the arterial supply indicating transit through the capillary network. If capillary perfusion pressure is not achieved, the casting resin will not transit through the capillary network and will not reach sinus supply. Additionally, evidence of failure to reach capillary perfusion pressure is the observation of casting material in the circle of Willis and the major arteries of the brain while observing an absence of resin at the sinuses and homogenous brain pigmentation. Under $40 \mathrm{X}$ magnification, penetrating arterioles and venioles were easily observed confirming complete transit through the vessel 
hierarchy (Supplemental Figure 5.1E). Our experience has taught us that it is imperative that the perfusion needle not to perforate into the right ventricle during the perfusion procedure. Perforation of the cardiac septum will allow resin to bypass arterial circulation and compromise perfusion pressure. Virtually, every instance of this resulted in poor quality of the vascular corrosion casts.

\section{Micro-Computerized Tomographic Imaging}

Tomographic images of the whole brain were acquired using a SkyScan $1272 \mu$-CT (Bruker). The $\mu$-CT system was set to operate at $35 \mathrm{kV}$ and $200 \mu \mathrm{A}$, no filter, and a 4032-by2686 frame size. Before initiating the image routine, flat fields were calibrated and the pixel size was set to $2 \mu \mathrm{m}$. To enhance the quality of acquired images, the voltage and exposure settings were optimized to a maximum transmission of $35-40 \%$, a minimum of $80-90 \%$, and an average of $65-75 \%$ through the sample. Each vascular cast was imaged over a total of $360^{\circ}$ at a step size of $0.05^{\circ}$ and averaged by 5 images per $0.05^{\circ}$. Each image routine required about 15 hours of scan time and produced a file size of 500GB containing 7,200 images and an additional 400GB and 4,032 images for the final coronal series of the entire cerebrovascular system. After completing the 15 hours scan, the primary data sets were corrected for misalignments, beam hardening, and ring artifacts then converted into a coronal image series. The final data set produced by this imaging routine is a complete coronal series of the entire cerebrovasculature at a pixel resolution of $2 \mu \mathrm{m}$ separated in the $\mathrm{Z}$ direction by $2 \mu \mathrm{m}$ for a total of 43.65 gigavoxels. 


\section{Anatomical Selection of Volume of Interest}

The complete set of coronal images was accessed on CTan (Bruker) to isolate a volume of interest (VOI). To prepare image sets for digital isolation of VOIs, the signal intensity of the image series was increased so that capillaries were easily observed. Once the intensity was increased, boundaries of major brain regions were clearly noticeable. Using the Allen Mouse Brain Atlas (Lein et al., 2007), the VOI was located and selected by tracing. The traced regions were made in square selections of approximately $1.5-2.0 \mathrm{~mm}$ and dynamically interpolated in the $\mathrm{Z}$ direction of $1.5-2.0 \mathrm{~mm}$. The result was a cube VOI that was saved as an individual data set. The new data set containing the VOI was filtered in three-dimensions to remove signal noise by implementing a filter that outputs an image based on the local averaging of the input image where all the values of the square kernel have the same weight. The data set was converted into a BMP series of formatted images and saved as a new data series of the isolated and pre-processed VOI.

\section{Image Processing and Optimization for Volume of Interest}

The workflow for the optimization of VOI data sets is depicted in Supplemental Figure 5.2. The binary VOIs were optimized for image analysis and quantification using ImageJ or Imaris software. Each VOI data set was three-dimensionally cropped to 500 x 500 x $500 \mu \mathrm{m}$ cube. The data sets were then rotated so that a selected landmark was co-registered by rigid transformation similar to the other data sets in the experimental group. Our experience dictates that the major arteries and their specific branching patterns provided the most convenient and consistent landmarks for image co-registration. The intensity of the data series was multiplied by 1.5 so that capillaries were easily seen; however, the signal of the most intense objects 
should not reach a pixel intensity of 255. After signal intensity multiplication, a threedimensional Gaussian blur was applied using a circular mask of 0.65 pixels. Then background was subtracted from the data by implementing a uniform noise filter that addresses the uniform noise that often contaminates digital images during acquisition. Please see (Pushpavalli \& Sivarajde, 2013) for a description of uniform noise and digital image processing. Next, the data set was filtered with an unsharp mask to increase the spatial resolvability of the structures in the data set. This new optimized data set was duplicated and saved as a series of tiff-formatted images. One of the duplicated data sets was used to create vessel centerlines that served to reduce errors during the vessel network-tracing algorithm. Centerlines were created using the 3D/2D skeletonization plugin on ImageJ. With the second duplicated image set, an intensity threshold was set using the Moments algorithm (W.-H. Tsai, 1985) and a binary image series was created. The intensity of the binary image set was subtracted by 150 so that the intensity of the binary image series was uniformly at 105. Finally, the image series containing the centerlines of the vessels was merged with the processed binary images so that the vessel structures had an intensity of 105 and the centerlines that passed through them contained an intensity of 255 (Supplemental Figure 5.3). This optimized data series was then filtered with a three-dimensional Gaussian blur and saved as a series of tiff-formatted images. For additional details on the post-processing of the image data and the specific parameters used in each of the functions, please refer to supplemental table 1. We calculated the signal-to-noise ratio (SNR) of the optimized images (Kennel et al., 2018) using ROIs by SNR $=20 * \log 10$ (Mean Signal / Standard Deviation of Background) to produce an estimate in decibels (dB). In addition, we calculated the SNR over an entire image using SNR $=20 * \log 10$ (Max Intensity - Minimum Intensity / Standard Deviation of ROI). Over 32 ROIs from individual images we estimated an 
average SNR of $34.29 \mathrm{~dB}(\mathrm{St} . \mathrm{Dev}=10.52, \mathrm{SEM}=1.86)$. From 10 separate images, we estimated an average SNR of $31.08 \mathrm{~dB}(\mathrm{St} . \mathrm{Dev}=3.40, \mathrm{SEM}=1.07)$.

\section{Volume of Interest Quantification and Analysis of Vascular Networks}

The optimized data sets were analyzed with Imaris (Bitplane) software and vascular networks were identified then quantified using the filament trace function. Filament trace allows the creation, visualization, editing, and the analysis of filament like structures in twodimensional and three-dimensional images. The settings for the algorithm used for filament trace were set to automatic filament and path detection. Filament trace will calculate the diameter of filaments from the image by approximating a circle of the cross section area. We used the loops algorithm during the tracing process and used the automatic threshold setting.

Filaments that were generated were automatically re-centered to the centerlines of the volumetric data set. Post filament trace, the vascular networks were visually inspected to ensure that proper vessel connections were made. Errors encountered following the filament trace procedure were exclusively to larger vessels with diameters greater than 15 microns. The type of errors that needed correction was gaps (smaller than vessel diameter) in an otherwise continuous vessel segment, which required manual connection. The second type of error that affected large vessels was improper junctions that occurred when two large vessels were in close proximity to each other. The segments added after manual connections were automatically recalculated for vessel diameter with Imaris Filament Trace algorithm (Supplemental Figure 5.4). Once a proper network was created, a number of statistics were generated and saved as excel sheets. These measurements include vessel diameter, length, volume, area, tortuosity, branch level, and angle in degrees. Vessels were classified by 
hierarchy that was determined by the mean vessel diameter. Vessels that had a mean diameter of $2-8 \mu \mathrm{m}$ were classified as capillaries, $9-34 \mu \mathrm{m}$ were arterioles and venioles, and $>34 \mu \mathrm{m}$ were classified as arteries and veins. To determine the effects of image noise on our threedimensional vascular network reconstruction and analyses, we created a synthetic data set with added artificial Gaussian noise at three levels of intensity then compared the measurements with a ground truth data set that was manually segmented and traced data (Supplemental Figure 5.5) (Corliss, Mathews, Doty, Rohde, \& Peirce, 2018; Kennel et al., 2018).

\section{Three-Dimensional Vascular Network Analysis}

To evaluate the cerebrovascular network, a network analysis was performed using a custom MATLAB script. The volumetric data sets were co-registered by rigid transformation. The pixel intensity of the co-registered data sets was globally multiplied by 1.5 so that the vascular structures were easily detected; however, the maximum pixel intensity of any data set was maintained below 255. A three-dimensional Gaussian blur filter with a circular mask of 0.65 pixels was applied to each data set. Then, the background noise is removed from each data set by subtracting the mean pixel intensity, calculated from a region equal to the diameter of the largest vessel then repeating this for all regions in a data set. To increase the spatial resolvability of the structures in the image series, the data sets were filtered with an unsharp mask. The data sets were then converted to a binary image series by selecting a threshold using the Moments algorithm. The binary data set was duplicated and converted into a local thickness map. The second binary data set was used to extract the centerlines of vessels. Our threedimensional network analysis processes the centerline data set and derives nodes as vertices with degree 3 or greater and edges as vertices with degree 2 and end-points as vertices with 
degree 1. Each edge is stored in a matrix containing all of its component pixel identities and the nodes that the edge bridges together. Then the local thickness map is used to characterize the network map for further analysis. The output data for the three-dimensional network analysis was a vascular map containing the parameters of the angioarchitecture for our assessment of network connectivity. The results of this analysis were used to assess the extensity of the vascular network by measuring the connectedness and branch abundance to the network.

\section{Three-Dimensional Data Rendering}

Our imaging device utilizes phase contrast enhancement allowing more information and detail from a single pixel at the detector. The final voxel size after phase contrast enhancement is dependent on the number of projections and the rotation step size of the acquisition routine. Traditional detector systems cannot measure directional information. Our tomographic detector system can collect information or deflection and scattering by measuring phase-shift information. This is important when considering that even very small objects can deflect $\mathrm{x}$-ray beams. This is why imaging $\mathrm{x}$-ray deflection is more sensitive for imaging small objects, rather than absorption alone. Unlike traditional absorption imaging, imaging deflection and scattering is not limited by the resolution of the system. Phase-shift information is stored as intensity for every pixel. During phase stepping, the system creates a sinusoidal modulation of intensity for every pixel of the camera. The loss of modulation at any specific pixel of the image reflects the object's local scatter. The system we used to acquire our image sets is capable of acquiring isotropic detail down to $0.35 \mathrm{um}$. Because of this principal, the reconstruction quality of our imaging data exceeded what would be expected at 2 um pixel size. 
To view the vascular network of the entire brain in three-dimensions, the data series was uploaded onto CTvox (Bruker) and the opacity and light intensity was adjusted to provide the best view of the data set. In order to demonstrate a color-coded three-dimensional structure of the vascular network, the data sets containing the VOIs were uploaded onto Imaris (Bitplane), rendered in three-dimensions, and colored based on calculated statistics. Colorcoding of the whole brain vascular network was performed on CTan (Bruker). Additionally, data sets were color-mapped based on vessel separation to allow the visualization of vessel density and intervessel distances.

\section{Group Size and Statistical Analysis}

All values are presented as mean \pm SEM for 5 female and 4 male brains. The specific statistical analysis used in each experiment was determined based on the intended comparison that was performed. Statistical tests were either student's t-test or ANOVA, with Bonferroni or Dunnett post-hoc tests. Specifically, Bonferroni's multiple comparison analysis was used to compare all combinations of the experimental groups, which include sex and brain subregion. Dunnett's post-hoc test was used for comparison of the experimental groups with a control group. Values of $\mathrm{p}<0.05$ were considered as significant.

\section{Results}

Vascular corrosion casts made by transcardial perfusion of a polyurethane resin (VasQtec, PU4ii) created a structurally precise and highly detailed replica of the cerebrovascular network of the entire brain in 3-month female and male mice. The creation of 
all vascular corrosion casts were performed within procedural parameters that preserved the native size and morphology of blood vessels. Female and male mice that were used in this study were 3.0 - 3.1 months of age, weighed an average of $30 \pm 2$ grams, and had an average body temperature of $36 \pm 0.2{ }^{\circ} \mathrm{C}$. In addition, mice that were used had systolic $(164 \pm 0.3)$, diastolic $(141 \pm 0.4)$, and a mean $(149 \pm 0.3)$ arterial pressures that were within the physiological range (Whitesall, Hoff, Vollmer, \& D'Alecy, 2004; Wilde et al., 2017). The duration for producing a single corrosion cast averaged 10-days. The casting procedure occurred in three phases: perfusion, cast processing, and tomographic imaging (Supplemental Figure 5.1A, C and E).

\section{Vascular Network Reconstruction}

Completed casts were mounted on Plexiglas pedestals with cyanoacrylate adhesive for tomographic imaging (Supplemental Figure 5.1F). MicroCT imaging routinely produced 4032 Bitmap images with dimensions of 4032 x 2688 pixels that encompassed the cerebrovasculature through the entire depth of the brain at a voxel size of $2 \times 2 \times 2 \mu \mathrm{m}$ encompassing 43.65 gigavoxels (Supplemental Figure 5.1G). Each data set was coregistered by the alignment of a combination of vascular landmarks and major subregions of the brain. The image quality produced by our reported image acquisition routine was superior; having essentially no signal-noise, imaging artifacts or intensity variations that are commonly encountered in fluorescence based imaging paradigms. Additionally, neither the loss or resolution nor the dissipation of signal intensity occurred to the internal structures of the brain, indicating that our acquisition routine achieved accurate and complete image collection of the vasculature through the entire thickness of the brain. With these data sets, we were able to 
reconstruct in three-dimensions the entire cerebrovasculature at one time, allowing for the rotation, inspection and clipping through the entire volume of the brain (Figure 5.1A). By general inspection of these reconstructed data sets, major subregions of the brain can clearly be identified by the changes in vessel density, hierarchy and organization (Figure 5.1A, panel 1-4). Reconstructed data sets in three-dimensions resulted in an anticipated organization of the cerebrovasculature. Briefly, the superior sagittal sinus and its neighboring pial vessels covering the surface of the cortex, the circle of Willis and its emanating major cerebral arteries (Figure 5.1B), hippocampal vascular networks in the characteristic hippocampal organization (Figure 5.1A, panel 2), the choroid plexus on the floor of the lateral ventricles (Figure 5.1A, panel 4), and the caudal rhinal vein (Figure 5.1C) can be identified.

Structure diameter maps were created for each data set allowing us to color code the hierarchy of vessels based on vessel diameter (Figure 5.2). Color coded images can be used to aid in navigating through the cerebrovasculature allowing a more intuitive positioning of volume of interest (VOI) boundaries for region specific analyses. Figure 5.3A depicts a reconstructed data set of the whole brain cerebrovasculature, color-coded to vessel diameter. Figure 5.3B depicts a digitally isolated VOI of the prefrontal cortex rotated to an anterior perspective, posterior perspective, and a toggled inward view. Figure 5.3C depicts a $500 \mu \mathrm{m}$ thick coronal slice of a brain hemisphere followed by sequential VOI selection and depiction of the primary somatosensory cortex, demonstrating the density of the vasculature and information encompassed by the data set. These data demonstrate the profound interconnectedness of the vasculature and its resulting emergence. 


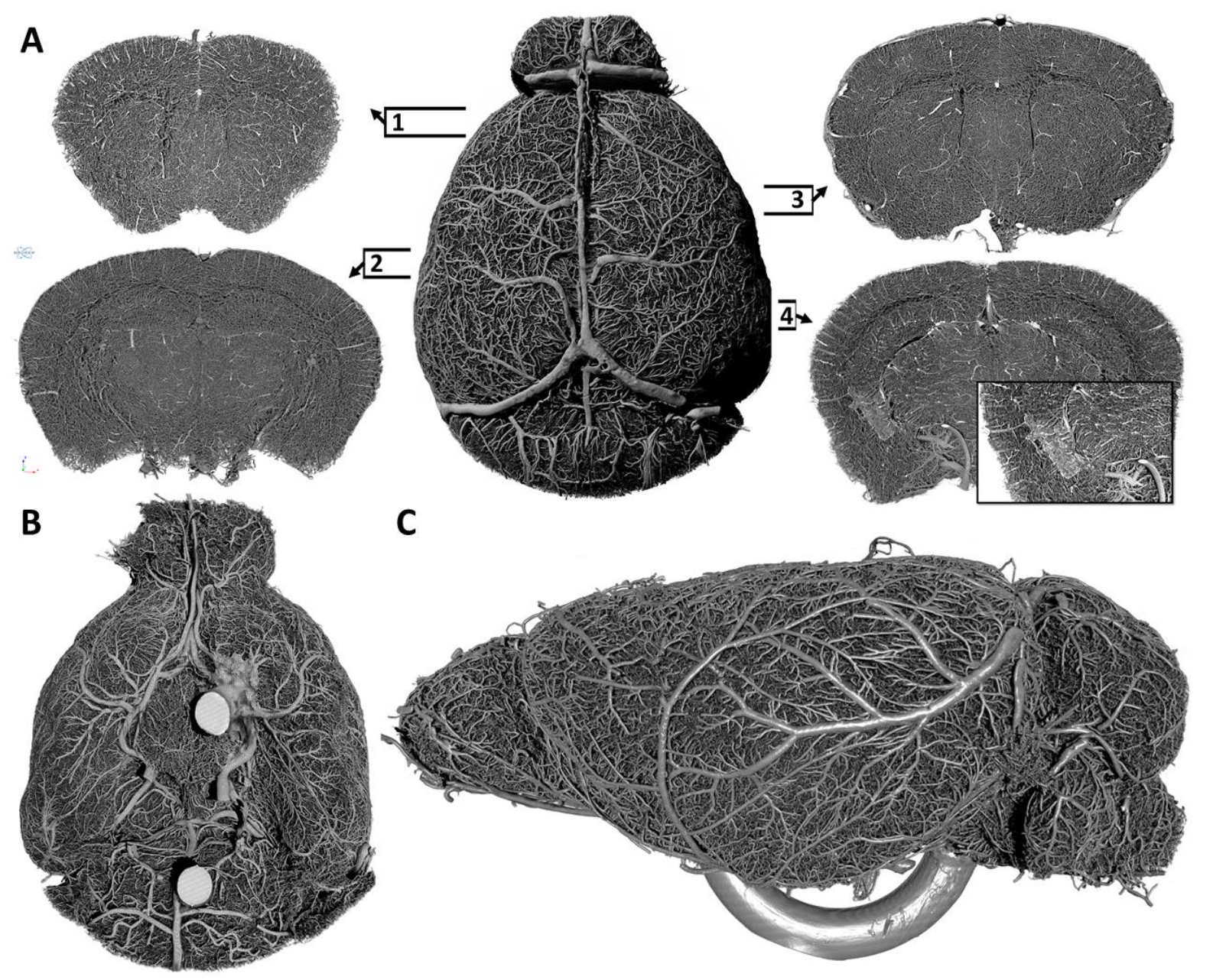

Figure 5.1. Three-dimensional reconstruction of the cerebrovascular network of the entire adult mouse brain. (A) Dorsal perspective of the whole brain vasculature (center) and selected $1 \mathrm{~mm}$ thick coronal slices of the volumetric image set to demonstrate the acquisition of imaging data through the entire thickness of the brain and to illustrate the profound density of vessels and the complexity of network connectivity at various brain subregions (1-4). Insert panel in slice four depicts the choroid plexus on the floor of the lateral ventricle. Numbered frames indicate the relative anterior-posterior position of each coronal slice. (B) Ventral perspective of the cerebrovasculature depicting the circle of Willis and the corresponding vascular network. (C) Lateral perspective of the cerebrovasculature depicting surface arterial and venous networks. (Panel B; intense circular structures, panel C; ventral U shaped structure = mount platform for cast). 
Whole Brain Analysis of the Angioarchitecture Demonstrate a Multitude of Vessels that are Morphometrically Similar between the Sexes

For whole brain vascular analysis (Figure 5.4, table), vessels were automatically identified and segmented by thresholding using the Ridler-Calvard method (Ridler \& Calvard, 1978). ROIs were created using a shrink-wrap routine that stretched over holes with a diameter of up to 30 pixels. The shrink-wrap routine allows for an automatic generation of ROIs by wrapping the boundary of the ROI around the periphery of a three-dimensional object represented in the data set. Selection of a threshold allows the boundary of the ROI to stretch over pores the size of the threshold in three-dimensional space so that the ROI traces the boundary of the represented object in the data set without tracing any pores or holes that the object may contain.

Two exceptions for a completely automated analysis, before automatic thresholding, the mean gray value of the data sets were manually derived and normalized to a value of 95 by global multiplication of pixel intensity.

Whole brain vascular analysis demonstrated a broad range of vessel diameters ranging from 2 $-220 \mu \mathrm{m}$, with a mean diameter of $36.6 \pm 2.4$ and $54.3 \pm 10.6 \mu \mathrm{m}$ for female and male mice, respectively. The data revealed that over the entire brain, the cerebrovascular network is composed of $1.4 \times 10^{6} \pm 2.6 \times 10^{5}$ and $1.8 \times 10^{6} \pm 3.0 \times 10^{5}$ vessel segments for female and male mice, respectively. The average intervessel distance in three-dimensions was $20 \pm 5 \mu \mathrm{m}$ in females and $32 \pm 13 \mu \mathrm{m}$ in males $(\mathrm{p}=0.037)$. Total volume of vessels in the female brain was $2.2 \times 10^{10} \pm 4.0 \times 10^{9} \mu \mathrm{m}^{3}$ and in males was $1.3 \times 10^{10} \pm 1.7 \times 10^{9} \mu \mathrm{m}^{3}(\mathrm{p}=0.053)$. The total volume 

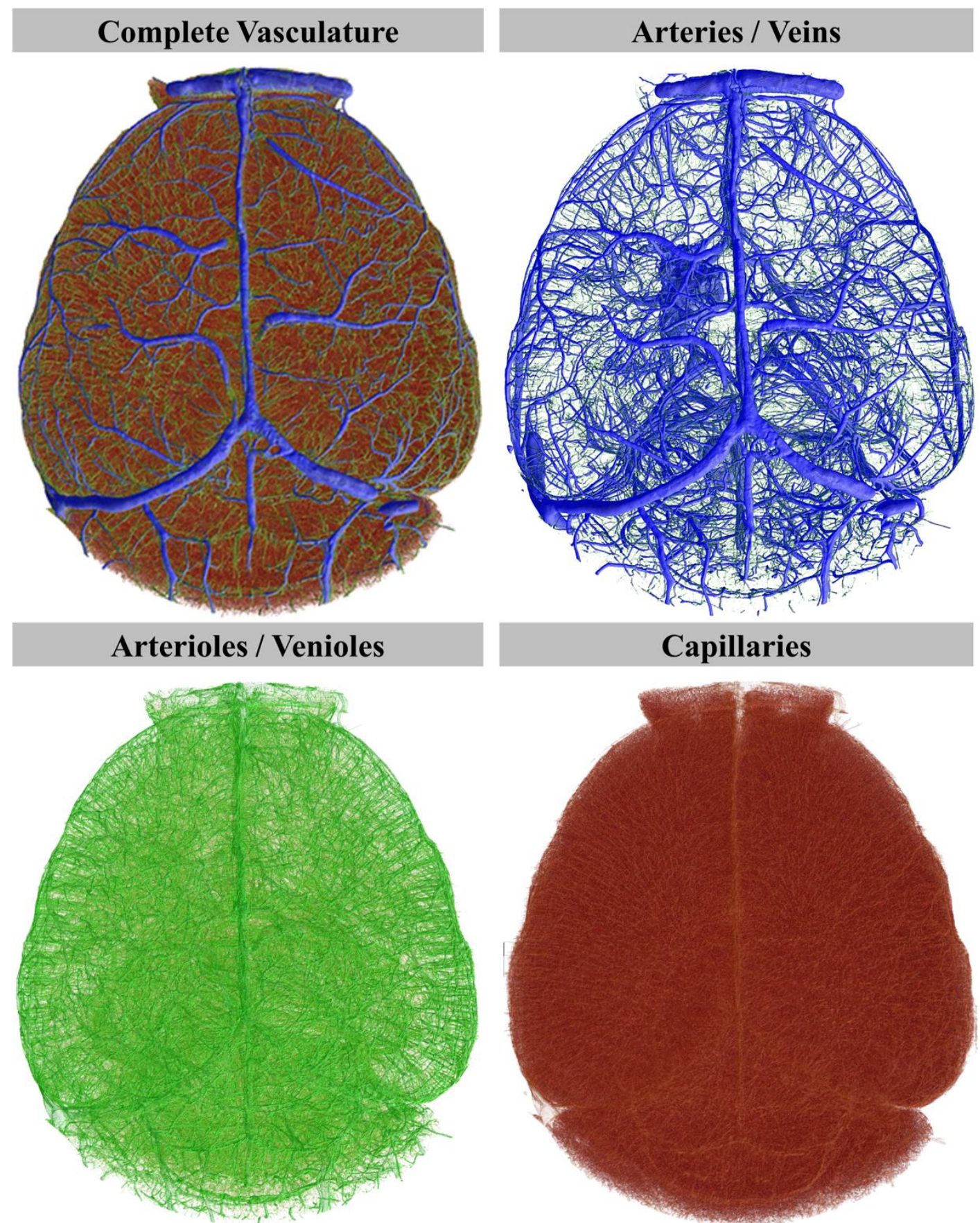

Figure 5.2. Whole brain vascular network segmentation and color-coding by vessel hierarchy demonstrates the distribution and frequency of vessels. Whole brain volumetric data set produced from a female mouse used to create a vessel thickness map to color-code vessels by hierarchy. Hierarchical classified vessels were migrated to hierarchyspecific volumetric data sets. 
of the brain parenchyma in microns cubed was $1.5 \times 10^{11} \pm 5.1 \times 10^{10}$ for females and $4.7 \times 10^{10} \pm$ $1.7 \times 10^{10}$ for males $(\mathrm{p}=0.065)$.

Although whole brain global-averages of the angioarchitecture in female and male mice appeared somewhat statistically indistinct from one another, specific differences at the level of vessel hierarchy may exist. We used the vessel thickness maps of the entire brain to classify vessels and analyzed vascular volume as a function of vessel diameter to provide a distribution of vessel species relative to the total mass of vessels per brain in female and male mice (Figure 5.4A and B).

We observed sex-related differences in the distribution of vessels of particular sizes with relatively low intra-group variability. For both female and male mice, vessels ranging from $2-15$ um in diameters represented the largest proportion of vessels among all vessel diameters. In females, this population of vessels represented roughly $50 \pm 6 \%$ of all vessels, whereas in males this population accounted for $40 \pm 3 \%$. Males were found to have a greater population of vessels with diameters larger than $15 \mu \mathrm{m}(\mathrm{p}=0.01)$, having $49 \pm 3 \%$ of vessels with diameters falling within $20-80 \mu \mathrm{m}$ in diameter. In females, only $36 \pm 3 \%$ of vessels within this range (Figure 5.4C).

Capillaries form networks varying in density that are acutely tailored to the specific metabolic requirements of the surrounding tissue. The plasticity of capillary formation and regression is crucial for dynamically active cells, such as neurons, where during periods of elevated activity they require capillaries to be closer and more numerous. Capillaries and other small vessels were more numerous in female than in male mice (Figure 5.4C). Because the density of capillary networks is influenced by localized neuronal activity, and the need to meet metabolic demand, we next asked whether there are sex-related differences in the distance 

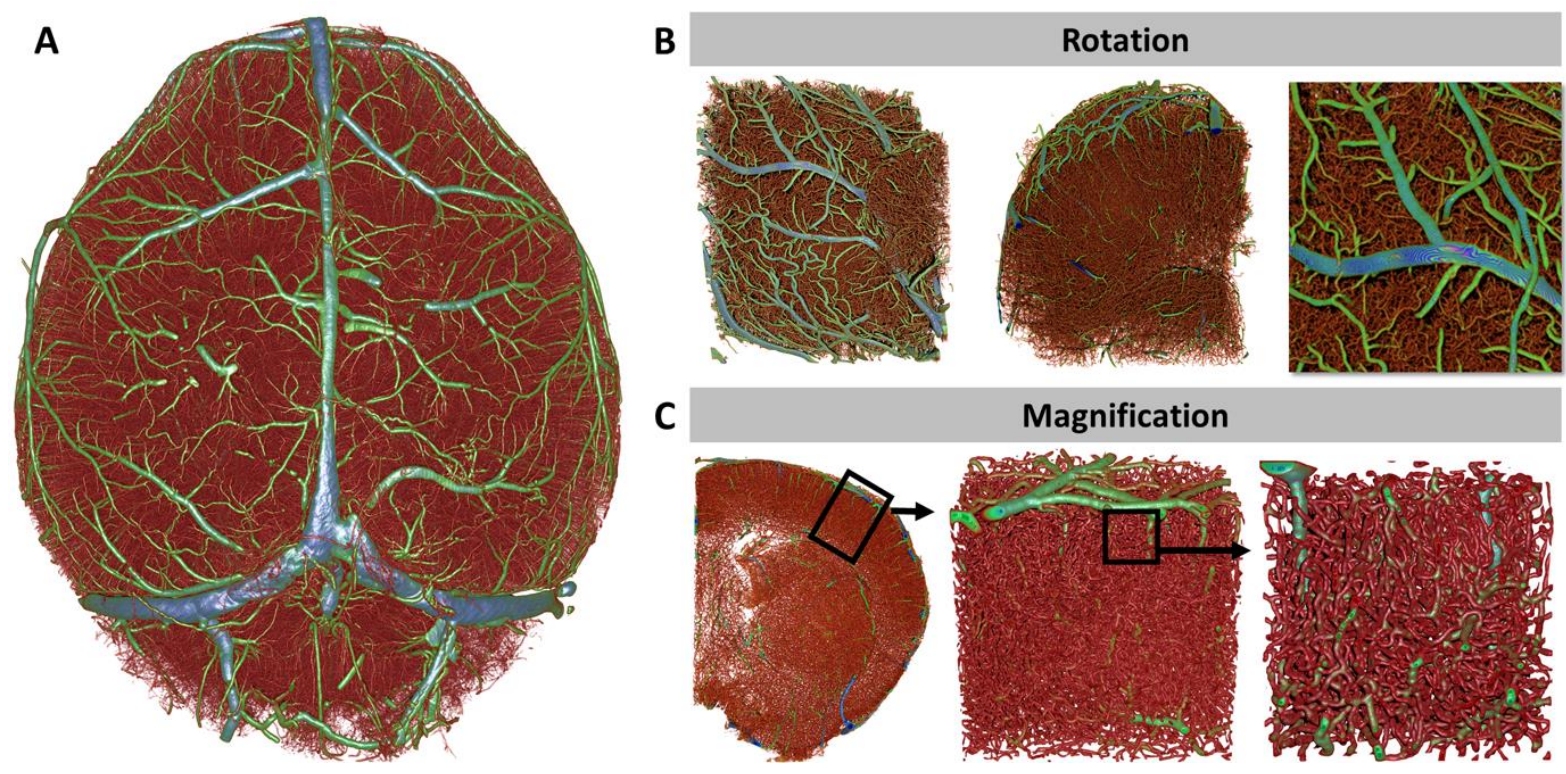

Figure 5.3. Large-scale volumetric data collection of intact cerebrovascular network at exceptional quality and resolution for microvascular network analysis. (A) Reconstructed whole-brain data set of the cerebrovasculature color-coded to vessel diameter. (B) Rotated sub-block of the right frontal pole to demonstrate the absence of morphological distortions that can be caused by aberrations of signal intensity. (C) Sequential magnification of the primary somatosensory cortex from a whole brain data set demonstrating the throughput and resolution of the collected data and depict microvascular networks that do not contain gaps or "missing" segment components that are often produced by confocal microscopy. Color-coded vessels from red (small) to blue (large). 
between vessels and by extension the distance between cells of the parenchyma and the nearest vessel. To examine this possibility, we analyzed the distance between vessels in threedimensions by measuring the radius of the largest sphere that fits between vessels (Figure 5.4D, insert). The radius of the sphere represents the maximum distance of any given cell in a particular parenchymal space is to the nearest vessel. A map of the distribution of parenchymal spaces as a function of maximum distance to the nearest vessel revealed a larger number of parenchymal spaces with shorter distances in male rather than female mice (Figure 5.4D).

Capillary networks form the interface for the exchange of molecules to and from the brain parenchyma and peripheral circulation. The total surface area of these capillaries is influential to the extent to which exchange can occur at the blood-brain barrier interface. To gauge the magnitude of this interface, we calculated the parenchymal volume to surface area ratio of capillaries. We found that the capillary surface area to tissue volume ratio of the whole brain to be $17.8 \pm 3.2 \mathrm{~mm}^{2}$ of capillary surface to every $\mathrm{mm}^{3}$ of brain tissue. Our estimate was in agreement with previous literature reporting the surface area of the blood-brain barrier in mice (Hartung et al., 2018) and in humans (Abbott, Patabendige, Dolman, Yusof, \& Begley, 2010; Pardridge, 2007) to be in range of $9-17 \mathrm{~mm}^{2}$ of capillary surface per $\mathrm{mm}^{3}$ of brain tissue.

\section{Whole Brain Analysis of the Vascular Network Topology Reveal an Array of Profoundly Interconnected Vessels}

We then examined the network properties of the whole brain vasculature. Consistent with the literature (Gross, 2006; Sheth \& Liebeskind, 2014), we observed a large prevalence of redundant pathways that formed a highly interconnected network. To measure the geometric 


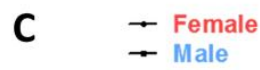

\begin{tabular}{|l|c|c|c|c|c|c|c|}
\cline { 2 - 6 } \multicolumn{1}{c|}{} & \multicolumn{2}{c|}{ Female } & \multicolumn{2}{c|}{ Male } & \multicolumn{2}{c|}{} \\
\cline { 2 - 7 } \multicolumn{1}{c|}{} & Average & SEM & Average & SEM & P-value & Units \\
\hline Total Brain Volume & $1.5 \times 10^{11}$ & $5.1 \times 10^{10}$ & $4.7 \times 10^{10}$ & $1.7 \times 10^{10}$ & 0.065 & $\mu \mathrm{m}^{3}$ \\
\hline Total Vascular Volume & $2.2 \times 10^{10}$ & $4.0 \times 10^{9}$ & $1.3 \times 10^{10}$ & $1.7 \times 10^{9}$ & 0.053 & $\mu \mathrm{m}^{3}$ \\
\hline Vascular Surface Area & $5.5 \times 10^{9}$ & $1.1 \times 10^{9}$ & $3.2 \times 10^{9}$ & $6.2 \times 10^{8}$ & 0.075 & $\mu \mathrm{m}^{2}$ \\
\hline Vessel Surface / Volume Ratio & 0.24 & 0.01 & 0.23 & 0.01 & 0.368 & $1 / \mu \mathrm{m}$ \\
\hline Average Vessel Diameter & 36.6 & 2.4 & 54.3 & 10.6 & 0.095 & $\mu \mathrm{m}$ \\
\hline Intervessel Distance & 19.9 & 5 & 31.8 & 13.5 & 0.037 & $\mu \mathrm{m}$ \\
\hline Fractal dimension & 2.6 & 0.05 & 2.5 & 0.06 & 0.052 & \\
\hline Number of Vessel Segments & $1.4 \times 10^{6}$ & $2.6 \times 10^{5}$ & $1.8 \times 10^{6}$ & $3.0 \times 10^{5}$ & 0.154 & Segments \\
\hline Volume of Parenchyma & $1.2 \times 10^{11}$ & $4.7 \times 10^{10}$ & $3.3 \times 10^{10}$ & $1.6 \times 10^{10}$ & 0.066 & $\mu \mathrm{m}^{3}$ \\
\hline Parenchymal Volume (\% Brain Volume) & 76.7 & 6.6 & 62.3 & 8.7 & 0.111 & $\%$ \\
\hline Euler number & $1.1 \times 10^{6}$ & $3.7 \times 10^{5}$ & $1.7 \times 10^{6}$ & $2.4 \times 10^{5}$ & 0.110 & Connections \\
\hline Connectivity (Redundancy) & $5.2 \times 10^{5}$ & $2.2 \times 10^{5}$ & $1.2 \times 10^{5}$ & $6.2 \times 10^{4}$ & 0.088 & Cuts \\
\hline Connectivity density & $4.2 \times 10^{-5}$ & $8.0 \times 10^{-6}$ & $3.7 \times 10^{-5}$ & $4.7 \times 10^{-6}$ & 0.333 & $1 / \mu \mathrm{m}^{3}$ \\
\hline
\end{tabular}

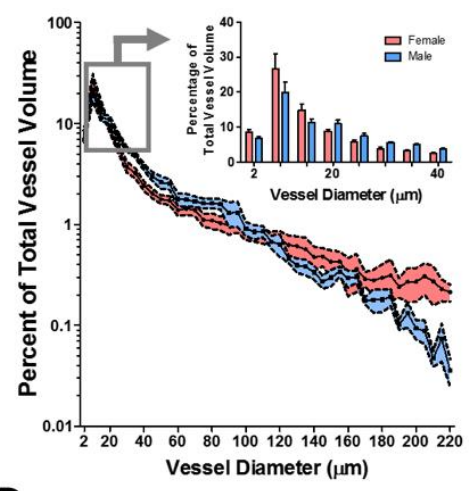

A

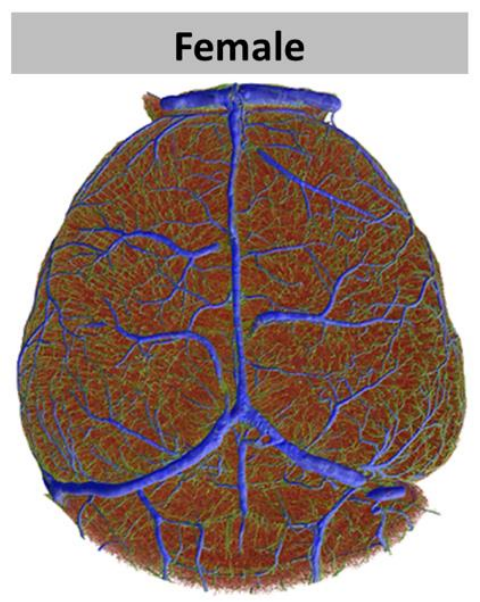

B

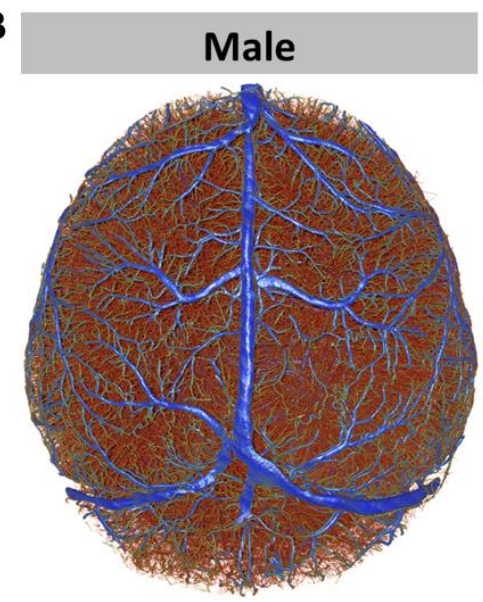

D

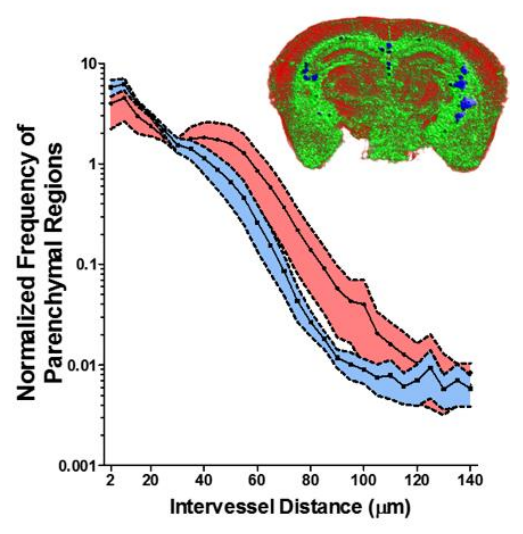

Figure 5.4. Topological analysis of the cerebrovasculature of the entire brain describes a sex-specific distribution of vessel frequency that is associated with vessel diameter and the frequency of parenchymal regions with intervessel distance. Cerebrovasculature reconstructed, parameterized (table), and color-coded by vessel diameter from (A) female and (B) male mice. (C) Line plot (mean \pm SEM) as a function of vessel diameter and the percentage of total vessel volume from female $(\mathrm{red}, \mathrm{n}=5)$ and male (blue, $n=4$ ) mice. The grouped bar graph inset depicts expanded data denoted by the box and arrow insert. (D) Line plot (mean \pm SEM) as a function of intervessel distance and normalized frequency of parenchymal regions from female (red, $\mathrm{n}=5$ ) and male (blue, $\mathrm{n}=$ 4) mice. The inset is a three-dimensional reconstructed coronal slab of the parenchyma that is color-coded from small (red) to large (blue) intervessel distance. To compare means, 2way ANOVA with Bonferroni multiple comparison analysis was used $\left(^{* *}, \mathrm{p}<0.01\right.$; ***, p $<0.001)$. 
connectedness of the network, we used an algorithm to calculate the Euler number and connectivity value of the network in three-dimensions. The Euler number takes into account three classes of events to estimate the total number of connections: islands, bridges, and holes. The connectivity number in a multiply connected network such as capillaries is topologically defined as the maximum number of cuts in the network that could be made without separating the network into two isolated networks (Nyengaard, 1999). Geometric analysis of the whole brain network revealed an Euler number of $1.1 \times 10^{6} \pm 3.7 \times 10^{5}$ connections in females and $1.7 \times 10^{6} \pm 2.4 \times 10^{5}$ connections in males. The connectivity value revealed $5.2 \times 10^{5} \pm 2.2 \times 10^{5}$ and $1.2 \times 10^{5} \pm 6.2 \times 10^{4}$ number of cuts to the network before splitting into two isolated networks in female and male mice, respectively.

Fractal dimension is a mathematical concept used to describe the complexity of selfsimilar structures. The prototypical idea of self-similar repeating functions originated as early as the 1600's but was only formalized in 1975 by Benoit Mandelbrot who coined the term fractal dimension. Briefly, fractal dimension analysis is used to parameterize structures with undefined functions. We measured network complexity using Kolmogorov box counting method for fractal dimensions and revealed a fractal dimension of $2.6 \pm 0.05$ and $2.5 \pm 0.06$ for female and male mice, respectively.

\section{Region of Interest Acquisition and Preprocessing}

To perform subregion specific vascular analyses, VOIs were digitally dissected from the whole brain data set. Positioning of VOIs was guided by the Allen Mouse Brain Atlas (Lein et al., 2007) and co-registered to landmarks comprised of arterial branching and positioning. Briefly, the medial orbital prefrontal cortex, cingulate gyrus, somatosensory cortex, corpus 
callosum, perirhinal cortex, and entorhinal cortex were isolated from the whole brain data sets (Figure 5.5). To increase the accuracy of the tracing process of the vessel network, the data sets containing the VOIs were optimized by inserting the centerlines of the vessels onto each z-plane of the same data set (Supplemental Figure 5.2). Imaris Filament Trace function computes pathways of the vascular network based on local pixel intensity of the vessels structure within the volumetric data set. The insertion of centerlines at the maximum pixel intensity of 255 , aids in this process by serving as the only local intensity at the highest pixel value of each vessel segment. Therefore, filament tracing by Imaris compute filament pathways based on the precise intersection of a vessel junction, negating errors caused by vessel segments in close proximity (close to touching) and spurious hair-like filament caused by improper setting of pathways due to irregular local intensity profiles.

\section{Region Specific Analysis of the Angioarchitecture Demonstrate a Highly Organized and Emergent Pattern}

Previous literature has demonstrated that in mice the primary somatosensory cortex receives the greatest vascular density compared to other brain regions (P. S. Tsai et al., 2009). In addition, there is a growing interest in understanding the neurovascular coupling involved in nuclear imaging and its relationship with blood-oxygen-dependent (BOLD) signal and neuronal activation (Magistretti \& Pellerin, 1999; Zhao, Wang, Hendrich, Ugurbil, \& Kim, 2006). Literature focused on the structural relationship of vascular networks in the somatosensory cortex (vS1) barrel field suggests that angioarchitecture of these networks are innately adapted to the topology of tissue (P. S. Tsai et al., 2009) and functional modules of the cortical columns in the vS1 barrel field (Blinder et al., 2013). We first assessed the 


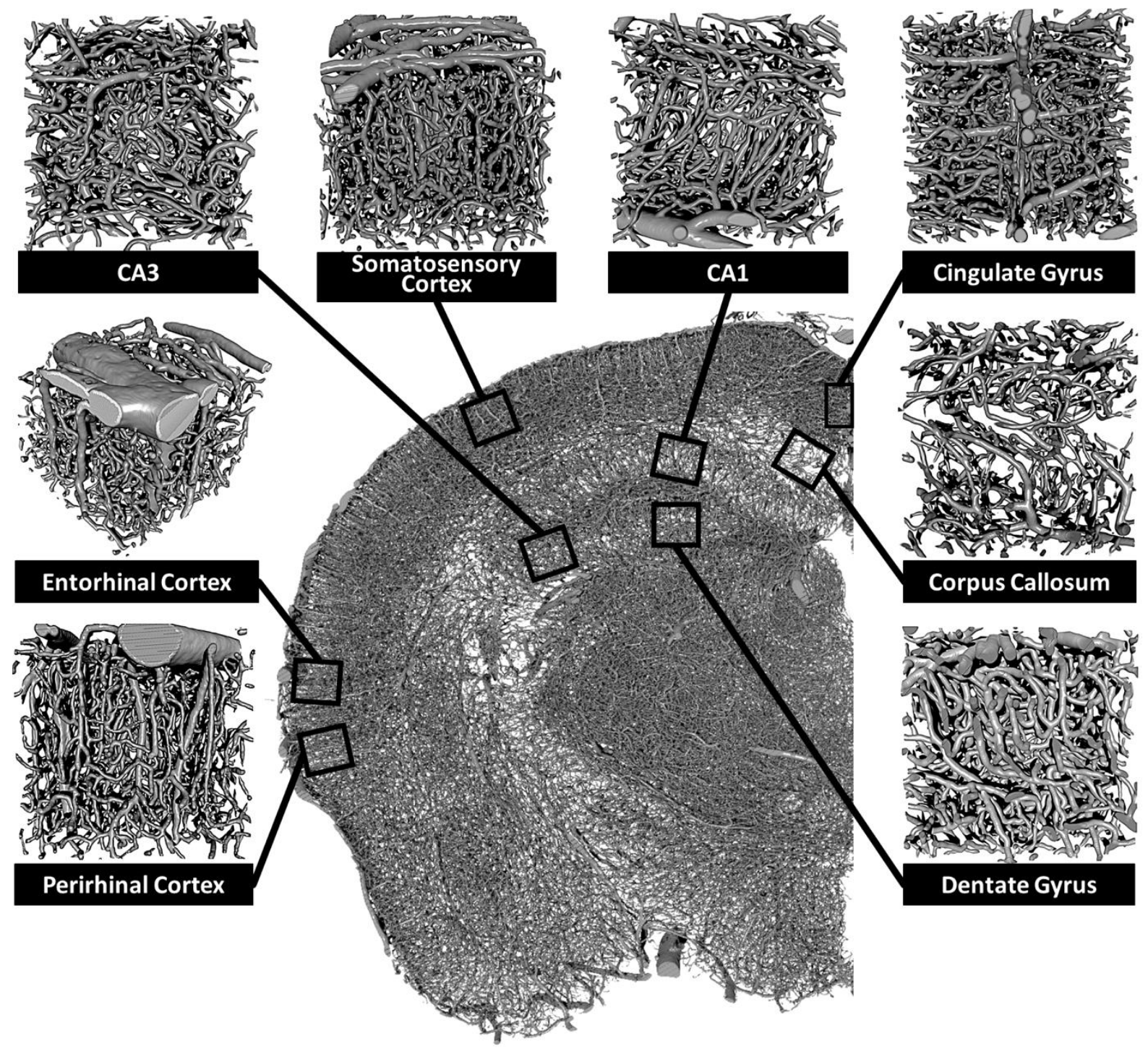

Figure 5.5. Identification and localization of brain subregions for morphometric analysis of the angioarchitecture. Three-dimensional reconstructed coronal slice of a single hemisphere with overlay box-and-stem insert demarcating the relative location of isolated perirhinal cortex, entorhinal cortex, CA3, somatosensory cortex, CA1, cingulate gyrus, corpus callosum, and dentate gyrus subregion. Subregion-specific vascular data sets were collected in 500 x 500 x 500 um selections. 
angioarchitecture of the primary somatosensory cortex by global morphometric analysis in female $(\mathrm{n}=5)$ and male mice $\left(\mathrm{n}=4\right.$, data set $\left.=500 \mu \mathrm{m}^{3}\right)$. Vessel density varied as a function of depth through the layers of the primary somatosensory cortex (Figure 5.6A). The greatest vascular density was found in cortical layer $2 / 3$ and the lowest density in layer $6 \mathrm{~b}$ (Figure 5.6B). The largest average vessel diameter was observed on the cortical surface and then remained constant throughout the depth of the cortex (Figure 5.6B). Morphometric and geometric analysis of the vascular network revealed modest sex-related differences in 3-month old mice (Figure 5.6C-K). Vascular analysis was performed on $660 \pm 54$ and $468 \pm 107$ vessel segments in female and male mice, respectively (Figure 5.6C). The calculated average over all vessel segments in the vS1 identified the average vessel diameter to be $14.76 \pm 0.22 \mu \mathrm{m}$ in

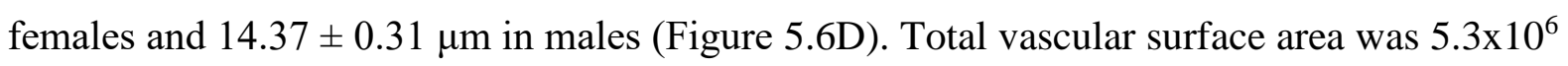
$\pm 5.5 \times 10^{5}$ and $4.5 \times 10^{6} \pm 3.3 \times 10^{5} \mu \mathrm{m}^{2}$ for female and male mice, respectively (Figure 5.6E). Vessels occupied a total volume of $1.7 \times 10^{7} \pm 2.1 \times 10^{6}$ and $1.4 \times 10^{7} \pm 1.4 \times 10^{6} \mu \mathrm{m}^{3}$ in female and male mice, respectively (Figure 5.6F). Whereas, parenchymal volume was observed at $1.0 \times 10^{8} \pm 2.1 \times 10^{6}$ and $1.1 \times 10^{8} \pm 1.4 \times 10^{6} \mu \mathrm{m}^{3}$ in female and male mice, respectively (Figure 5.6G). Comparable to the literature (Meyer et al., 2008; P. S. Tsai et al., 2009; Zhao et al., 2006), we observed an average distance between vessels in the somatosensory cortex to be $38.6 \pm 2.4 \mu \mathrm{m}$ in females and $43.1 \pm 1.5 \mu \mathrm{m}$ in males $(\mathrm{p}=0.10)$ (Figure $5.6 \mathrm{H})$. Similar to the average intervessel distance of the whole brain, female rather than in male, demonstrated smaller distance between vessels.

We next extrapolated on the global calculations of total vascular volume and parenchymal volume by mapping the distribution of vessel and parenchymal space diameter as a function of total volume (Figure 5.6I). We found that female relative to male mice had a 
greater volume of vessels with a diameter of $15 \mu \mathrm{m}$, occupying a volume of $6.5 \times 10^{6} \pm 1.2 \times 10^{6}$ and $3.8 \times 10^{6} \pm 7.7 \times 10^{5} \mu \mathrm{m}^{3}(\mathrm{p}<0.01)$ in female and male mice, respectively (Figure 5.6J). However, when these values were normalized to the percentage of total vascular volume, female mice have greater proportional volume of vessels with diameters that range $5-10 \mu \mathrm{m}$ than male mice $(\mathrm{p}<0.001)$. We then examined parenchymal separation volume to estimate vascularization of the primary somatosensory cortex. To assess somatosensory cortex vascularization, we mapped the distribution of parenchymal separation in three-dimensions as the maximum diameter of an inscribed sphere per parenchymal area as a function of total volume (Figure 5.6I). Our parenchymal separation analysis revealed a slightly larger total volume of parenchymal spaces with diameters that ranged from $10-30 \mu \mathrm{m}$ in females compared to males (Figure 5.6K). In contrast, males demonstrated a larger total volume of parenchymal separation with diameters ranging $40-55 \mu \mathrm{m}$. Although the distribution of areal parenchymal diameter as a function of cumulative volume provides an adequate description of the topological composition of the parenchyma that touches on the variation of parenchymal compartmentalization, a more direct assessment of the variation of minimum-distances of parenchymal cells to neighboring vessels is needed. We resolved this by examining the average minimum distance within a parenchymal volume and repeated this for all parenchymal zones. Our analysis revealed a heterotypic distribution of shortest distances between female and male mice. Female mice were found to have a greater number of parenchymal zones with smaller average minimum distances than male mice. Specifically, female mice demonstrated $3.2 \times 10^{4}$ $\pm 6.5 \times 10^{3}$ parenchymal zones with an average minimum distance below $15 \mu \mathrm{m}$, whereas male mice contained $2.4 \times 10^{4} \pm 2.7 \times 10^{3}$ parenchymal zones with distances below $15 \mu \mathrm{m}$. Furthermore, parenchymal zones with an average minimum distances of $15 \mu \mathrm{m}$ showed the 


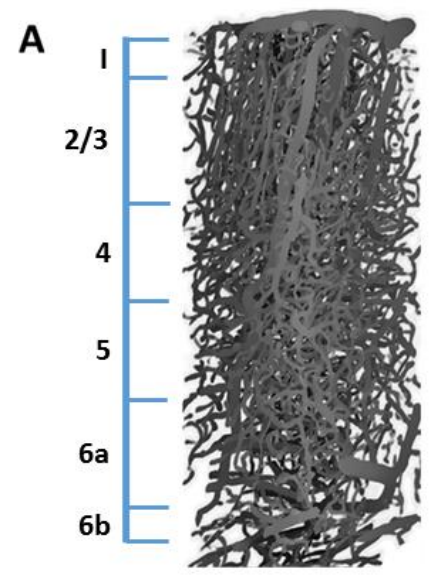

C

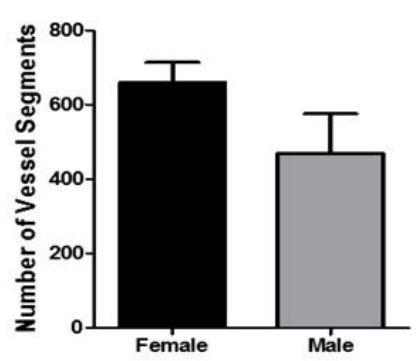

F
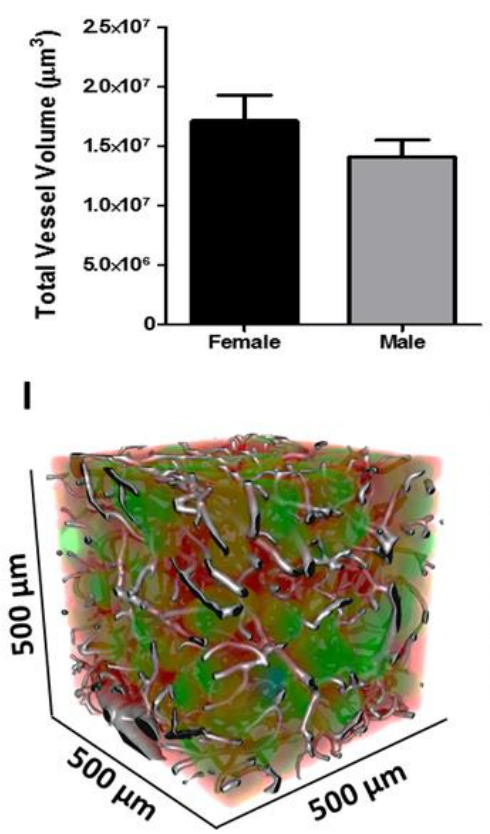

B

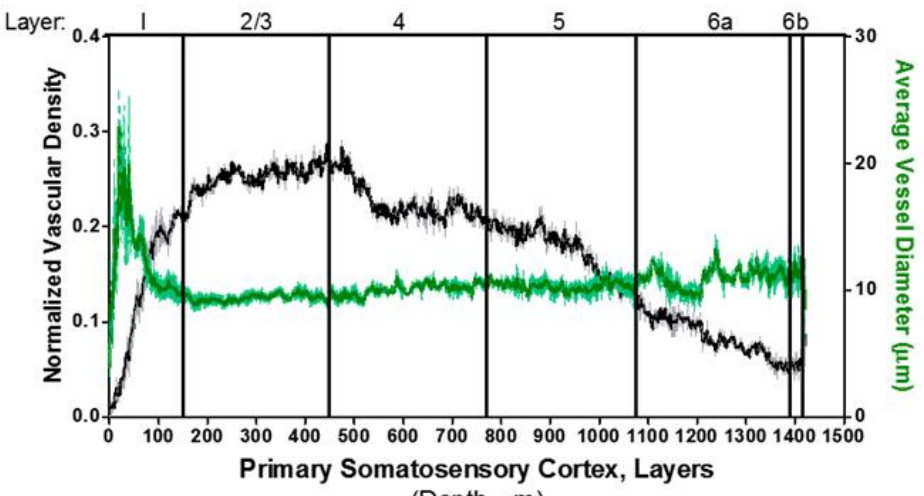

D (Depth, $\mu \mathrm{m}$ )

E
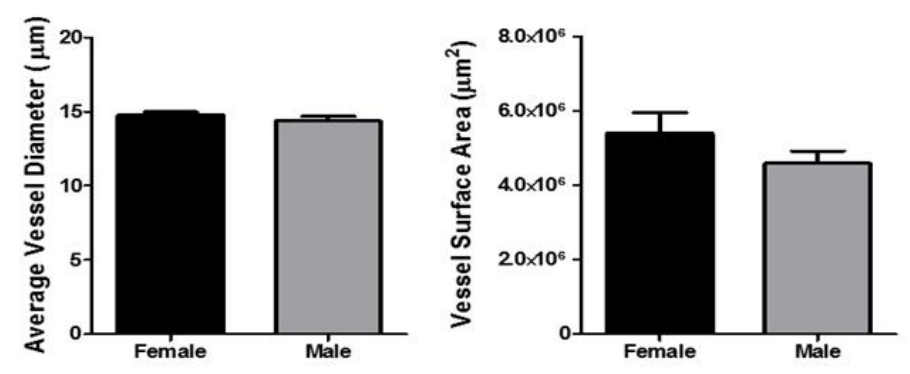

H
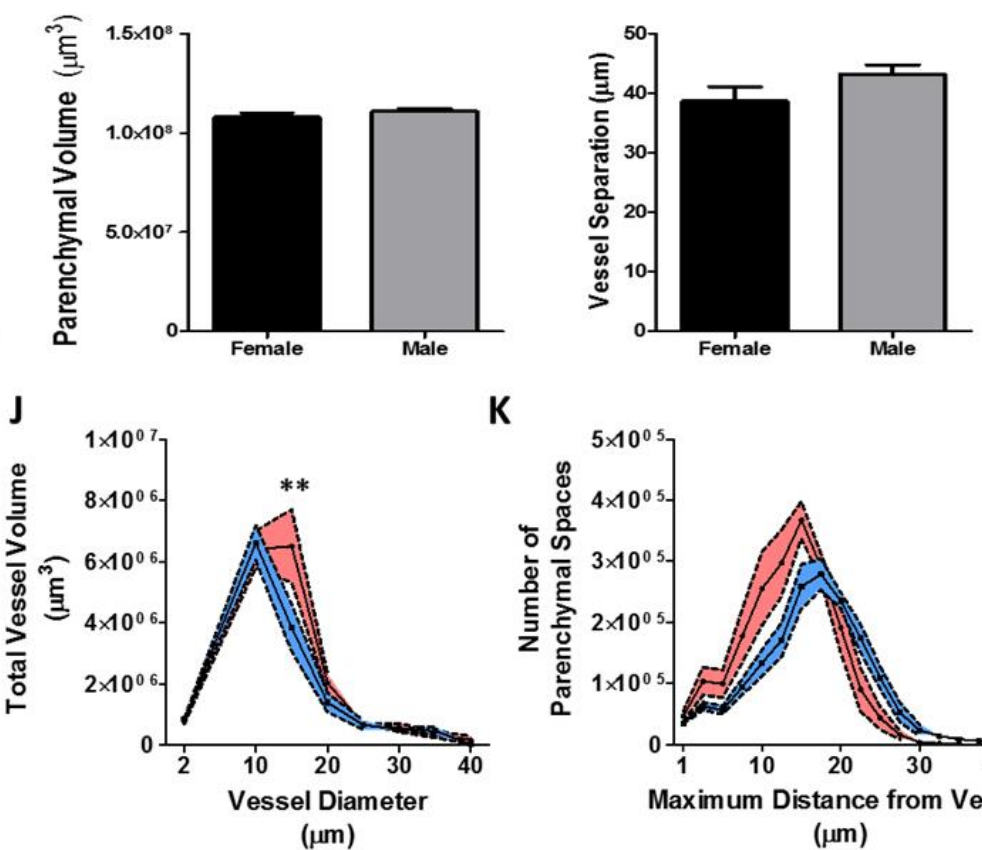

K

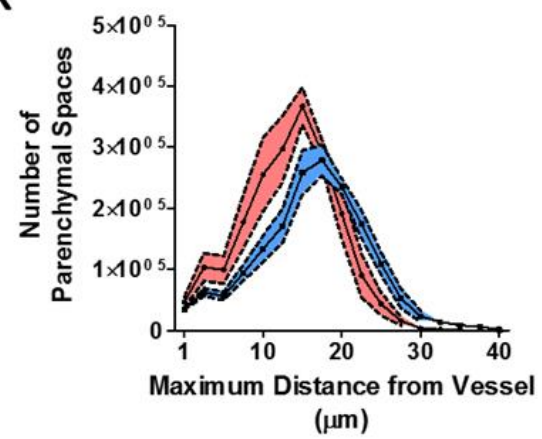


Figure 5.6. The primary somatosensory cortex demonstrates a topological distinct vascular network. (A) Reconstructed vascular network through the entire depth of the somatosensory cortex (500 x 500 x $1450 \mathrm{um}$ ) from a female mouse. Line insert demarcates the boundaries of the cortical layers. (B) Line graph (mean \pm SEM) as a function of cortical depth and normalized vascular density (black, $n=3$ ) or average vessel diameter (green, $n=$ 3). Bar graph(s) (mean \pm SEM) illustrating the (C) number of vessel segments, (D) average vessel diameter, (E) vessel surface area, (F) total vessel volume, (G) parenchymal volume, and $(\mathrm{H})$ vessel separation from female $(\mathrm{n}=5)$ and male $(\mathrm{n}=4)$ mice. (I) Reconstructed volumetric data set containing the vasculature from the primary somatosensory cortex merged with the parenchymal image series color-coded to the distance between vessels. Line graph(s) (mean \pm SEM) as a function of $(J)$ vessel diameter with total vessel volume and $(\mathrm{K})$ maximum distance from vessel with the number of parenchymal spaces. Statistical comparison was performed by 2-way ANOVA and Bonferroni post hoc test to compare the means $(* *, p<0.01)$. 
largest discrepancy $(\mathrm{p}=0.04)$ between female $\left(3.6 \times 10^{5} \pm 3.0 \times 10^{4}\right)$ and male $\left(2.5 \times 10^{5} \pm\right.$ $3.6 \times 10^{4}$ ) mice and accounted for the majority of all parenchymal zones in both male and female mice (Figure 5.6K). Our estimates are in agreement with the predictions reported in the literature (P. S. Tsai et al., 2009).

\section{Region Specific Analysis of the Vascular Network Topology Reveal a Conserved Network Organization and Connectivity that Covaried with Vessel Hierarchy}

The vascular network is highly interconnected and may asymmetrically form connections based on vessel hierarchy and diameter (Figure 5.7A). We would expect the vascular network to have asymmetric connectivity with a greater number of junction points at the capillary level due to their physiological relevance of distributing the flow of blood. As such, a penetrating arteriole can have a number of branch points along its length whereas capillaries branch at their distal segment (Figure 5.7B). In contrast, a network with symmetric connectivity should demonstrate a constant value of junctions across varying segment diameters (symmetric distribution). To determine whether the vascular network has asymmetric connectivity, we calculated the number of branch points as a function of parent vessel diameter (26,435 branch points across 9 mice) (Figure 5.7C). We then compared this to the estimated symmetric distribution of branch points and found that all vessels statistically differed from a symmetric distribution $(\mathrm{p}<0.001)$ except for vessels with diameters of 3 and $7 \mu \mathrm{m}$ (Figure 5.7D). We next compared the total number of branch points per average vessel diameter across all other average diameters to determine whether a hierarchy or characteristic vessel diameter forms proportionally greater number of interconnections. We found that vessels with diameters of 4 and $6 \mu \mathrm{m}$ represented $57 \pm 1.5 \%$ of the total population of vessel- 
vessel connections. Specifically, $21.5 \pm 2.2 \%(\mathrm{p}<0.0002)$ and $35.5 \pm 1.9 \%(\mathrm{p}<0.001)$ of all vessel junction points were found on vessels with diameters of 4 and $6 \mu \mathrm{m}$, respectively. This observation agrees with our prediction that the vascular network of the primary somatosensory cortex has asymmetric connectivity where capillaries are the primary vessel hierarchy that bridge together to form networks. Additionally, our data imply that among capillaries there is an asymmetric distribution of connections that interacts with specific capillary caliber preferential to diameters of 4 and $6 \mu \mathrm{m}(\mathrm{p}=0.000005)$. We next compared the distribution of branch points by parent vessel diameter across sexes to uncover any sex specific differences in network connectivity. We first compared the total number of branch points over all vessel diameters in the primary somatosensory cortex and found no statistical difference across sex (Figure 5.7E). If we average both female and male mice together, we obtain approximately 2937 branch points which is 7\% less than reported in the literature (Blinder et al., 2013). Thus, we then hypothesized that between male and female mice there would be no difference in network connectivity in relation to vessel diameter and number of branch points. Our null hypothesis is that there are sex-dependent differences in network connectivity that are related to network component diameter. To assess this we used an equivalence test to determine if the overall distribution of branch points across vessel diameters were similar between the female and male mice and found statistically significant similarity with $95 \%$ confidence that the distributions were equivalent (Pearson $R=0.95 ; \mathrm{p}<0.0001 ; \mathrm{R}^{2}=0.91$ ). Although we found the overall distribution to be equivalent between female and male mice, we next asked whether the magnitude of network connectivity preferential to 4 and $6 \mu \mathrm{m}$ vessels were similar. This was determined via multiple comparison analysis using a 2-way ANOVA and a Bonferroni post hoc test for level of significance and found that females (1835 \pm 490$)$ over males (1082 \pm 
102) demonstrated a greater number of branch points for vessels with an average diameter of $6 \mu \mathrm{m}(\mathrm{p}<0.01$; Figure 5.7F). These data indicate that in mice regardless of sex, the composition of the vascular network follows a conserved organization of connectivity in respect to capillary diameter. Furthermore, our finding that female mice have a greater number of branch points from vessels with $6 \mu \mathrm{m}$ diameters suggests that the differences in whole brain angioarchitecture between the sexes were influenced primarily by an elevated number of capillaries with $6 \mu \mathrm{m}$ diameters.

Penetrating arterioles deliver blood to subsurface microvascular networks, which drain into the penetrating venioles. There are no pathways to transit blood out of the brain that bypass the capillary network. Arteriovenous anastomoses that form in the brain that are pathological in nature confound the perfusion of blood into capillary beds by rerouting blood flow through a malformed arteriole that feeds blood directly into the corresponding veniole. In a healthy brain, there are a profound number of pathways for blood flow to reach the nearest veniole (Figure 5.7G). Blood flow can be regulated by modulating the resistance of these pathways individually to redistribute blood by shunting blood flow away or toward specific areas. The capillary angioarchitecture provides redundant pathways for the delivery of blood from any arteriole (Figure 5.7H). This network redundancy provides multiple direct pathways for blood to transit into and out of a given region (Figure 5.7I).

To quantify the number of connections within the local vascular network of the primary somatosensory cortex, we used an algorithm to calculate the Euler number and connectivity value of the three-dimensional vascular network and found an average of $310 \pm 66$ connections in female and male mice together. A comparison of the number of connections in the primary somatosensory cortex in female and male mice revealed $361 \pm 67$ and $226 \pm 142$ connections 
$(p=0.18)$ in females and males, respectively (Figure 5.7J). We next measured the redundancy

of the vascular network by using an algorithm that randomly cuts the network repeatedly until the network splits into two isolated components. We found that the vascular network of the primary somatosensory cortex could receive $504 \pm 95$ cuts before breaking apart, regardless of sex. Specifically, our analysis revealed as many as $587 \pm 134$ and $366 \pm 95$ cuts to the vascular network of the primary somatosensory cortex before splitting into two isolated networks in female and male mice $(\mathrm{p}=0.14)$, respectively (Figure $5.7 \mathrm{~K})$. These findings demonstrate that the vascular network of the primary somatosensory cortex are profoundly interconnected forming as many as 310 connections able to withstand the removal of 503 segments before network breakdown. Moreover, these observations imply that there is no difference in the interconnectedness of the vasculature between female and male mice.

\section{Sex Differences in Intragroup Interregional Angioarchitecture}

Within the brain, there exist subregion-dependent differences in vascular network organization and density. The specific architecture of the vascular network is closely tied to the local metabolic demand. As such, in gray matter regions, the energy requirement is relatively greater than in regions of white matter (Attwell \& Laughlin, 2001; Magistretti \& Pellerin, 1999), therefore vascular network density is increased in these zones. Brain structure and organization of neurons differ between subregions. For example, the cortex and hippocampus form layers and these layers demarcate zones in which specific cells can be found. In addition, differences in neuronal activity between brain subregions require specific angioarchitecture. This implies that within the cortex both anatomical organization of cells and local cellular activity within a cortical layer or between cortical layers can influence the local 

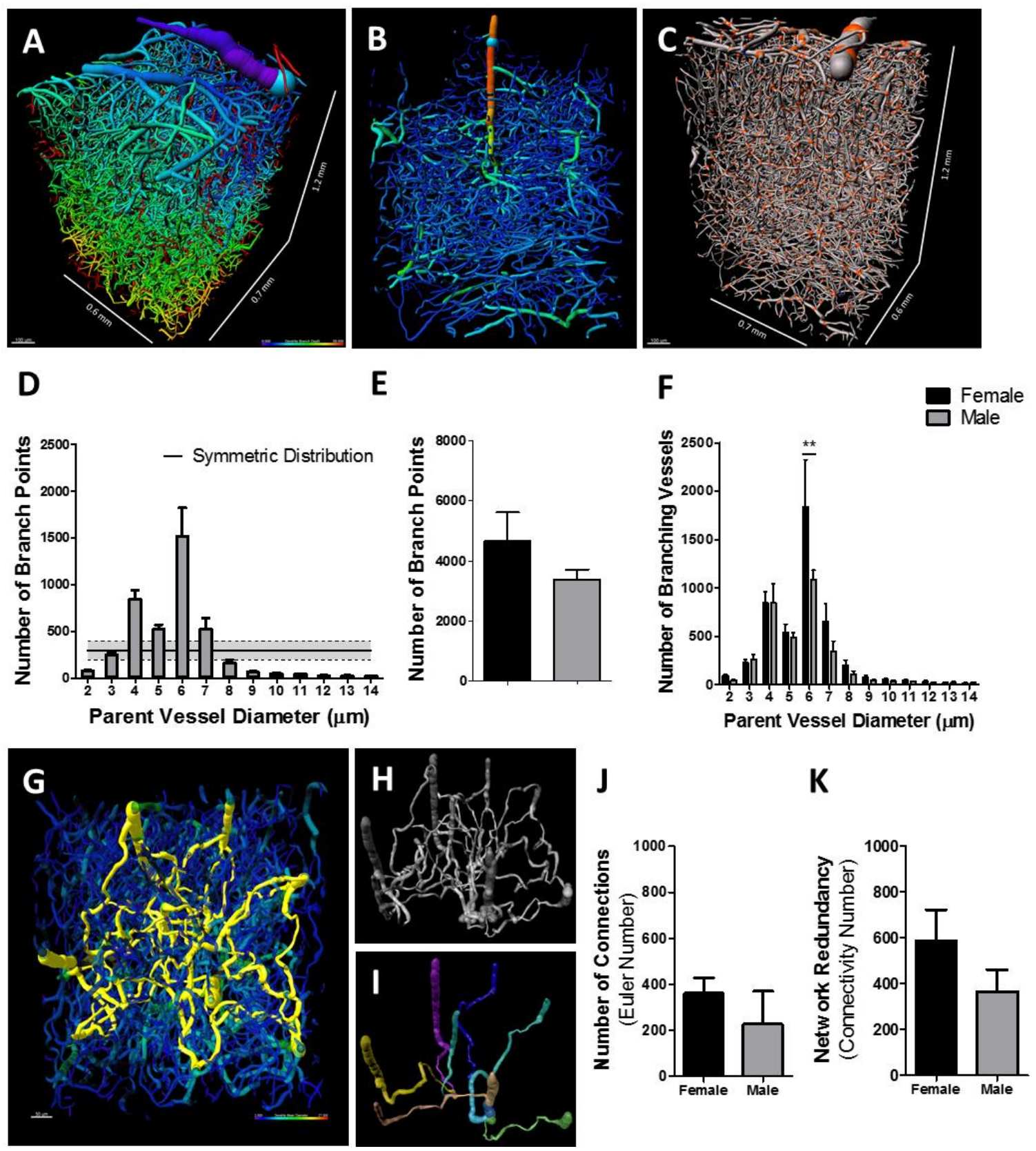


\section{Figure 5.7. Vascular network connectivity analysis in the primary somatosensory}

cortex indicates a preference for interconnections to specific vessel hierarchy. Vascular network through the depth of the somatosensory cortex (A, C) and single penetrating arteriole (B), color-coded to cortical depth (A), vessel diameter (B), and demarcated branch points (C, orange spheres). Bar graph(s) (mean \pm SEM) depicting parent vessel diameter as a function to number of branch points (D), total number of branch points (E), and parent vessel diameter as a function to number of branching vessels $(\mathrm{F})$ in female $(\mathrm{n}=5)$ and male $(n=4)$ mice. Dorsal perspective of a vascular network depicting the shortest pathways of each vessel to all other vessels (yellow) (G). Non-connected vessels segments are colored green and blue. Representative image of the extracted shortest pathways between vessels predicts the network at its most rudimentary form $(\mathrm{H})$. Depiction of the shortest pathways of each arteriole (green, cyan, blue, magenta, yellow, brown) to a veniole (beige), demonstrating the most effective pathway for blood to transit through penetrating arterioles and feed into venioles (I). Bar graph(s) (mean \pm SEM) demonstrating the number of vesselto-vessel connections $(\mathrm{J})$ and network redundancy $(\mathrm{K})$ from female and male mice. To compare means, 2-way ANOVA with Bonferroni multiple comparison analysis was used $(* *, \mathrm{p}<0.01)$. 
organization and density of the vascular network. This suggests that the topology of the vascular network throughout the brain is highly heterogeneous yet somewhat predictable by its acute responsiveness to local demand of blood perfusion. The goal of this analysis was to describe the magnitude of inter-subregion differences of the cerebrovascular topology. This analysis was achieved by means of geometric assessment of the vasculature then by pairwise comparing these metrics across subregions (Figure 5.8). We then assessed the magnitude of inter-subregion differences between sexes. The analysis uses intra-group differences in the angioarchitecture then compares the degree of heterogeneity across sex, to provide a metric of the diversity of vascular network parameters in female and male mice.

Subregion-specific vascular network differences can occur through hierarchy specific angiogenesis. Therefore, we categorized vessels by binning into two groups, vessels with diameters of 2-8 and 9-20 $\mu \mathrm{m}$ for capillaries and arterioles/venioles, respectively. For each data set, the total length of vessels that fell into either bin was summated (Figure 5.8A-B) and compared by 1-way ANOVA across subregions (medial orbital prefrontal cortex, somatosensory cortex, cingulate gyrus, and the dentate gyrus) or students t-test for comparing across the overall average of the subregions. The degree of difference was represented as the p-values per pairwise comparison (Figure 5.8C-F). We observed the highest density of capillaries in the primary somatosensory cortex versus other brain regions (female, $\mathrm{p}=0.0005$; male, $\mathrm{p}=0.0001)$. The second highest capillary density was found in the medial orbital prefrontal cortex (female, $\mathrm{p}=0.0005$; male, $\mathrm{p}=0.0001$ ). Whereas, lower densities were found in the cingulate gyrus (female, $\mathrm{p}=0.003$; male, $\mathrm{p}=0.002$ ) and dentate gyrus (female, $\mathrm{p}=$ 0.003; male, $\mathrm{p}=0.02$ ). Relative to all other regions, the lowest vascular density was found in the dentate gyrus for both female and male mice. A comparison of the total capillary length in 
the cingulate gyrus between female and male mice indicated a greater total length of capillaries in male mice (Figure 5.8A; $\mathrm{p}=0.02$ ). Our finding is consistent with the literature in that the highest capillary density is found in the primary somatosensory cortex in rodents (Harrison, Harel, Panesar, \& Mount, 2002).

In contrast we found the highest arteriole/venioles density in the cingulate gyrus (female, $\mathrm{p}=0.02$; male, $\mathrm{p}=0.001$ ) and dentate gyrus (female, $\mathrm{p}=0.2$; male, $\mathrm{p}=0.005$ ) and the lowest in the medial orbital prefrontal cortex (female, $p=0.02$; male, $p=0.0004$ ) and primary somatosensory cortex (female, $\mathrm{p}=0.2$; male, $\mathrm{p}=0.001$ ) in both sexes. Comparison of the sum length of arterioles in the medial orbital frontal cortex indicated that females have a greater value than males (Figure 5.8B; $\mathrm{p}=0.01$ ).

Our data indicate clear network differences between the subregions. However, our comparison of subregional differences to the global average is useful to identify a region that contains relatively elevated or reduced densities, but fails to demonstrate differences or similarities in a region-by-region manner. Therefore, we compared the total capillary length of all subregions to all other subregions to assess region similarities and differences (Figure 5.8C and E). We then performed the same comparison for arteriole/veniole densities across regions (Figure 5.8D and F). We found that the capillary density in the medial orbital prefrontal cortex and in the somatosensory cortex significantly differed compared to all other regions and most strongly differed from the cingulate gyrus and dentate gyrus in both female (Figure 5.8C) and male (Figure 5.8E) mice. In contrast, we found that the capillary density in the cingulate gyrus and in the dentate gyrus contained similar density profiles $(\mathrm{p}=0.820)$ while being significantly different from the medial orbital prefrontal cortex $(\mathrm{p}=0.01)$ and primary somatosensory cortex $(\mathrm{p}=0.001)$ 
A

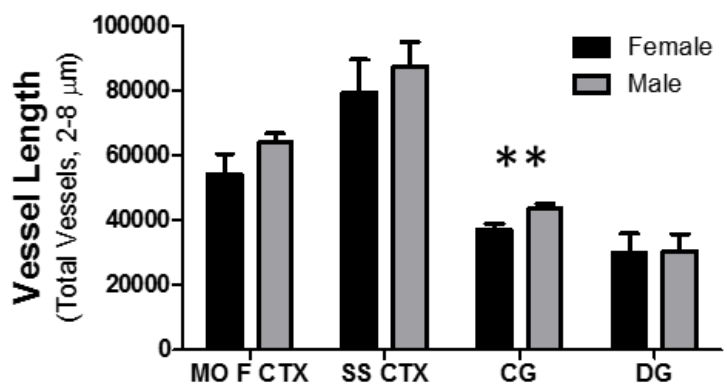

C

FC

2 - 8 um Diameter

SS

CG

DG

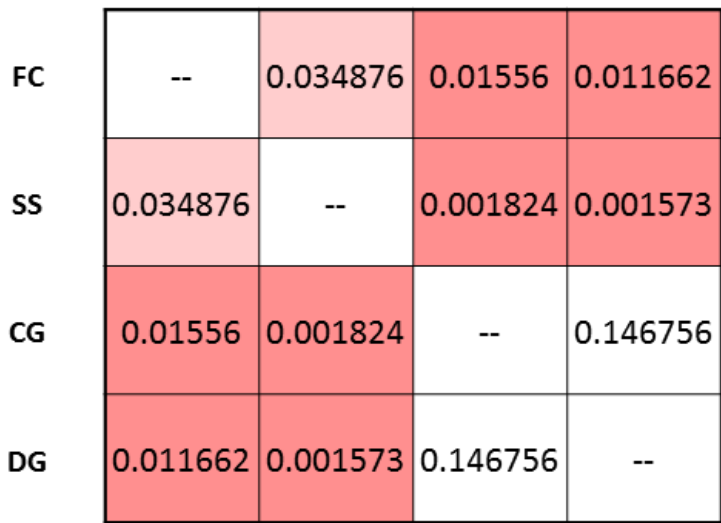

E

2 - 8 um Diameter

FC

SS

CG

DG

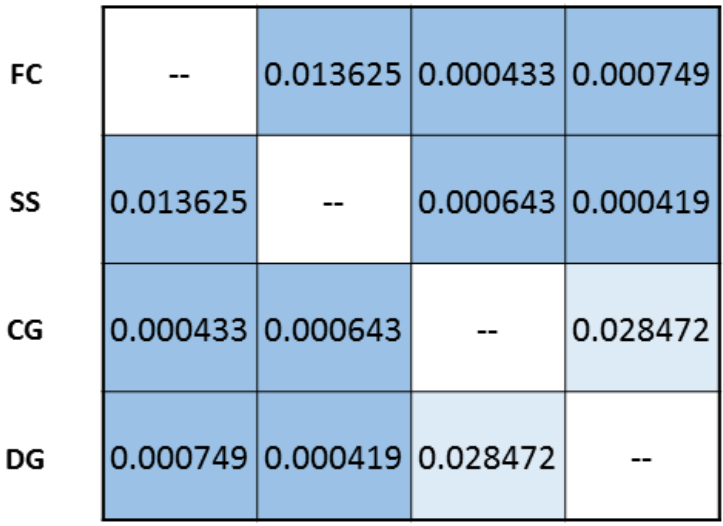

B

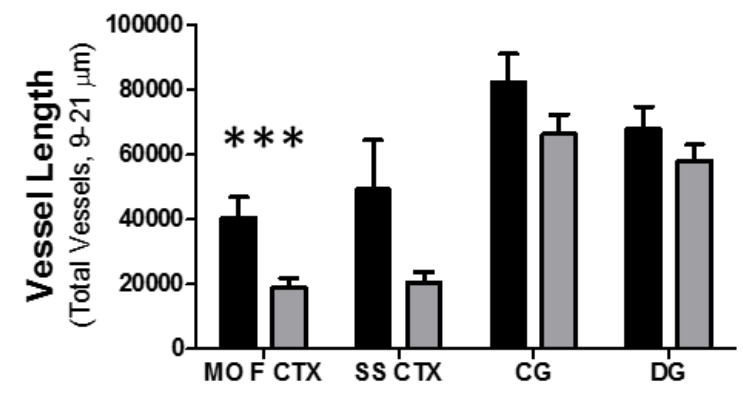

D

9 - 20 um Diameter

$\begin{array}{llll}\text { FC } & \text { SS } & \text { CG } & \text { DG }\end{array}$

FC

SS

CG

DG

\begin{tabular}{|c|c|c|c|}
\hline-- & 0.304671 & 0.002339 & 0.01032 \\
\hline 0.304671 & -- & 0.047421 & 0.149556 \\
\hline 0.002339 & 0.047421 & -- & 0.113585 \\
\hline 0.01032 & 0.149556 & 0.113585 & -- \\
\hline
\end{tabular}

$\mathbf{F}$

9 - 20 um Diameter

$\begin{array}{llll}\text { FC SS } & \text { CG }\end{array}$

\begin{tabular}{l|c|c|c|c|}
\cline { 3 - 5 } FC & -- & 0.374484 & 0.000205 & 0.000276 \\
\cline { 2 - 6 } SS & 0.374484 & -- & 0.000275 & 0.000397 \\
\cline { 2 - 6 } CG & 0.000205 & 0.000275 & -- & 0.166258 \\
\cline { 3 - 5 } DG & 0.000276 & 0.000397 & 0.166258 & -- \\
\cline { 2 - 5 }
\end{tabular}


Figure 5.8. Inter-subregional differences in the vascular architecture vary in magnitude between the sexes. Grouped bar graph (mean \pm SEM) depicting (A) total length of capillaries $(2-8 \mu \mathrm{m})$ and (B) total length of non-capillaries $(9-21 \mu \mathrm{m})$ in micrometers for each of the brain subregions. Inter-subregional analyses of the differences in total $(\mathrm{C}$ and E) capillary and (D and F) non-capillary length in (C and D) female and (E and F) male mice represented as mean $\mathrm{p}$ - values color by hue intensity to the level of significance. Brain subregions (500 x 500 x 500 um): medial orbital prefrontal cortex (MO F CTX), primary somatosensory cortex (SS CTX), cingulate gyrus (CG), dentate gyrus (DG). To compare means, 2-way ANOVA with Bonferroni multiple comparison analysis was used $(* *, \mathrm{p}<0.01 ; * * *, \mathrm{p}<0.001)$. 
The comparison of arteriole/venioles profiles between subregions revealed group wise differences between the medial orbital prefrontal cortex and primary somatosensory cortex compared to the cingulate gyrus and dentate gyrus. In female mice the greatest disparity was found between the medial orbital prefrontal cortex and the cingulate gyrus $(p=0.002)$ or the dentate gyrus $(\mathrm{p}=0.01)$. In males, the medial orbital prefrontal cortex showed a greater dissimilarity compared to the cingulate gyrus $(\mathrm{p}=0.0002)$ and dentate gyrus $(\mathrm{p}=0.0002)$. Similarly, in males, there was also strong dissimilarity between the primary somatosensory cortex and the cingulate gyrus $(\mathrm{p}=0.0002)$ or the dentate gyrus $(\mathrm{p}=0.0003)$. Interestingly, both female and male mice demonstrated similar arteriole/venioles profiles between the medial orbital prefrontal cortex and primary somatosensory cortex (female, $\mathrm{p}=0.3 ;$ male, $\mathrm{p}=0.3$ ) and between the cingulate gyrus and the dentate gyrus (female, $p=0.1$; male, $p=0.1$ ).

\section{Discussion}

We demonstrate a procedure for the acquisition and analysis of the entire brain cerebrovascular system at a microvessel-relevant resolution and provide a detailed characterization of the geometrical and topological properties of the vessels and their networks in the adult female and male mouse brain.

We used a vascular corrosion cast method to replicate the complex structures of the cerebrovasculature (Figure 5.1). We describe a procedure that yields with high fidelity, a structurally precise and extensively detailed replica of the network of cerebral vessels through complete depth of the adult mouse brain (Supplemental Figure 5.1). Our imaging paradigm yields a single volumetric data set that encompasses the entire brain at a resolution required to 
investigate microvasculature (Figure 5.3). The produced data sets were of high quality, essentially devoid of non-specific signal (background) or imaging aberrations. Reconstruction of the cerebrovasculature from the volumetric tomographic data sets demonstrated the capture of the geometry of microvascular networks that were seemingly absent of segment breakage and disconnected components. We report in detail the analytical models employed to investigate the structural and connective complexity of the brain vasculature from nine mice.

When performed under idealistic conditions, we would expect the procedure to produce a vascular cast that contains all vessels of the entire adult mouse brain. However, some limitations should be noted. First, although polyurethane is one of the most thermally, mechanically, and chemically resilient polymers in production we have to consider potential damage to the cast during processing. Particularly, small structures such as microvessels can break and potentially tangle if the cast is improperly handled or if the cast is disturbed before complete curing of the resin. Once the resin has cured, the elasticity and durability of polyurethane makes the manipulation of the cast simple (Krucker, Lang, \& Meyer, 2006). Earlier methods to produce corrosion casts used methyl methacrylate resins, which are exceptionally brittle, and rigid (Hodde \& Nowell, 1980). Handling of the polyurethane cast prior to and after maceration is less critical than with methyl methacrylate resin. Second, imaging on a desktop MicroCT system requires the cast to be mounted in such a way to prevent movement artifacts caused by vibration or shifting of the specimen. This can prove to be difficult because mounting in any form requires contact with the cast. This contact, if not maintained as minimal as physically possible can have an impact on analysis and measurements of vessels at the location of the cast-mount interface. Third, the time required from the creation of a cast to final analysis is large. Alone, image acquisition at the resolution 
described in this study requires over 15 hours of continuous scan time, in addition, analysis of a single data set of the whole brain requires over 18 hours of computer processing. While there are several limitations to consider when applying a corrosion cast to research, if performed correctly and carefully, the advantages clearly outweigh its limitations.

The convoluted nature of the cerebrovascular system requires the use of appropriate mathematical models to represent accurately its geometry, organization, and functional properties. Geometric descriptions of the brain's vascular system have proven useful in the literature (Cassot et al., 2010; Cassot, Lauwers, Lorthois, Puwanarajah, \& Duvernoy, 2009). Indeed, geometric analysis of the vascular system provides valuable insight to fundamental physiological features such as, branch characteristics and cumulative length (Lauwers, Cassot, Lauwers-Cances, Puwanarajah, \& Duvernoy, 2008). However, commensurately important is a description of the vessel network organization using non-integrated parameters. In mathematics, the concept of topology is used when describing an object beyond its geometrical features, rather focusing on its structural connectivity. Topological analysis of the vasculature has only recently emerged in the literature. The reason for this delay is the unavailability of large data sets at the resolution required for robust statistical analyses of the cerebrovasculature (Hirsch, Reichold, Schneider, Székely, \& Weber, 2012). Knowledge of the topology of the cerebrovasculature is critical for understanding the principals of neurovascular coupling and for functional predictions such as, the differential impact of a vessel occlusion at different locations and vessels in the brain. Progress made on describing the interplay between vascular network topology and layer-specific differences in blood flow, resistance, and pressure gradients has provided an essential perspective of the contribution of vascular network topology to the local regulation of blood flow in the brain (Blinder et al., 2013; Gould, Tsai, 
Kleinfeld, \& Linninger, 2017; Guibert, Fonta, \& Plouraboué, 2010; Lyons, Parpaleix, Roche, \& Charpak, 2016; Schmid, Tsai, Kleinfeld, Jenny, \& Weber, 2017). Such studies have revealed that the relationship between the topology of a vascular network and its distribution of blood and pressure is so fundamentally linked that it is argued to be one entity (Schmid et al., 2017).

In this study, we use both geometrical and topological analysis to describe the morphology and connectivity of the angioarchitecture that represents its structural properties in a physiologically meaningful manner (Supplemental Table 5.2).

There are sex-related differences in cerebral blood flow and metabolism. It is well accepted that females have greater cerebral blood flow than do males (Aanerud, Borghammer, Rodell, Jónsdottir, \& Gjedde, 2017; Amen et al., 2017). However, extensive research also supports the notion that males have a thicker cortex and higher synaptic density than do females (Alonso-Nanclares, Gonzalez-Soriano, Rodriguez, \& DeFelipe, 2008). Both notions are well accepted in the field yet seem counterintuitive. Having a thicker cortex with a greater number of synapses would intuitively suggest a greater metabolic demand and a corresponding increase of cerebral blood flow. Our data could resolve this conflict by providing insight into the sex differences of the cerebrovascular system; we report our findings that the entire brain vascular network in female mice contains proportionally greater capillary networks than do males (Figure 5.4C). This discrepancy is likely caused by an elevated number of branching vessels that are $6 \mu \mathrm{m}$ in diameter in female mice (Figure 5.7F). However, in contrast to our integrated parameterization of the whole brain, when we consider subregion-specific differences between the sexes we encounter the opposite effect to variable degrees. This is best illustrated in our analysis of the vasculature in the cingulate cortex, in which we observed males to have a greater total length of capillaries than do females (Figure 5.8A). The increased capillary content in the 
cingulate cortex of male mice is likely sexually dimorphic and is the result of an underlying difference in subregion activity (Lawal, Kern, Sanjeevi, Hofmann, \& Shaker, 2005; Liu, Zubieta, \& Heitzeg, 2012). Furthermore, we find that within the sex, subregional vascularization can be similar to other subregions. We interpret these observations to suggest that brain subregions with similar vascular profiles may demonstrate commensurate activation. Furthermore, similarities and dissimilarities of the vascular profile between subregions were conserved in both female and male mice. Importantly, observing no differences in integrated vascular parameters between the sexes does not indicate that the networks are homotopic. For instance, in the primary somatosensory cortex, our analysis revealed a lack of differences in total length of capillaries between the sexes (Figure 5.8A) indicating commensurate capillary vascularization that intuitively suggests homotopic networks. However, when we analyzed the branching properties of these networks we found a dissimilarity of vessel connectivity that specifically propagated from capillaries with a diameter of $6 \mu \mathrm{m}$ (Figure 5.7F) which indicates incongruence of the deconstructed network and heterotopy of the capillary network.

Importantly, we observed cerebrovascular parameters that were similar regardless of sex. These similarities provide us with information of a conserved network at the species level. Analysis of vascular network organization and hierarchical involvement in the formation of network connections demonstrated a precise relationship between parent vessel diameter and number of branching vessels that were similar in both sexes (Figure 5.7F). This informs us that network formation follows specific rules where its population of vessel components exist and interact differently, and is governed by its hierarchical vessel diameter. Interestingly, we observed two peaks in the distribution of number of branches by parent vessel diameter (Figure 5.7D). These peaks represented branches emanating from parent vessels with 4 and 6 microns 
diameters. Although, female mice were found to have a greater number of 6 microns vessel branching, the distribution proportional to other parent vessel diameters was the same between female and male mice. Parent vessels with diameters of 7 microns tended to be greater in females. All other parent vessel diameters were nearly identical between female and male mice. These observations are interpreted to imply that network formation has specific organization where it follows a conserved set of rules that pertain to vessel diameter and connectivity. Specific branching patters that follow a diameter-related organization may be physiologically important for healthy hemodynamic function. It would be interesting to determine if changes in these branching properties of vascular networks occurs during pathology.

The cerebrovascular system is a complex network of highly interconnected pathways that maintain physiological hemodynamic states of blood delivery to support the perpetual activity of neuronal transmission. The consistency and precision of the forces and amount of blood delivery to the various brain regions is critical for normal information processing of neurons (Douglas \& Martin, 2004). Our analysis of whole brain network complexity supports the notion that females more so than males demonstrate complex vascular organization. Fractal dimension is a mathematical concept used to describe the complexity of self-similar structures. In contrast to topological analysis, measuring fractal dimension provides an index that quantitatively and qualitatively describes the difference of a structure's space filling behavior compared to an orthodox geometric structure. In vascular biology, analysis of fractal dimension is used to measure network complexity (Cassot, Lauwers, Fouard, Prohaska, \& Lauwers-Cances, 2006; Lorthois \& Cassot, 2010). The quasi-fractal structure of vascular networks is produced by its hierarchical branching patterns (i.e. Figure 5.7B) that scale many iterations to give rise to progressively smaller vessels (Lorthois \& Cassot, 2010; Peyrounette, 
Davit, Quintard, \& Lorthois, 2018). In this case, the term quasi-fractal is used to denote the combination of fractal and non-fractal characteristics of vessel networks. For a greater description of quasi-fractal geometry of vascular networks, see (Cassot et al., 2006; Lorthois \& Cassot, 2010; Smith et al., 2019). Our fractal analysis of the whole brain vasculature indicated a fractal dimension of $2.6 \pm 0.05$ and $2.5 \pm 0.06$ in female and male mice, respectively. Our observation is in agreement with the literature, reporting a fractal dimension of 1.82 and 2.17 of the vasculature in subcortical and cortical areas, respectively (Cassot et al., 2006). It is important to note, the relationship between space-filling properties of a structure's organization with its derived fractal dimension is a measure of the structure's complexity in terms of its self-similar repetitive nature and the degree of regularity to which its repeated structure propagates through multiple scales. It is incorrect to assume that an object's fractal dimension and the relationship to space filling properties is a measurement of its density. Qualitatively, the fractal dimension of a vascular structure gauges its branch patterns by assessing the uniformity between its branches of like hierarchical order and the number of branch levels the network produces (i.e. how extensive are the branches in the network and do these branches resemble parent vessel morphology through multiple subsequent branch levels?). Our data suggest that the branching properties in the female brain are more organized and thorough, and are therefore more complex than in male mice.

Vascular network complexity can influence the outcome severity following an ischemic event. Premenopausal women and intact female rodents sustain smaller infarctions than males (Alkayed et al., 1998; Faber, Moore, Lucitti, Aghajanian, \& Zhang, 2017). Some studies argue that sex differences of ischemic stroke sensitivity are caused by gonadal hormones (Manwani et al., 2015). During normal aging in females, sexually dimorphic 
capillary density and network organization may decline with gonadal hormones resulting in increased sensitivity to ischemic injury. In menopausal women, collateral rarefaction ensues, resulting in increased severity of ischemic injury (Li \& Chi, 2011; Moore, Zhang, Maeda, Doerschuk, \& Faber, 2015). One study, comparing the number of collateral vessels in female and male mice with the outcome of ischemic injury found no statistical difference in the number of collateral vessels between the sexes while observing smaller infarct volumes in female mice (Faber et al., 2017). Our data demonstrates a greater total number of capillaries, more extensive capillary-capillary network, and smaller intervessel distances in female mice than in male mice. In consideration of these findings, we argue that female mice have more complex and robust vascular infrastructure that reduces ischemic sensitivity compared to male mice. Moreover, microvascular density rather than number of collateral vessels may cause the sex differences in sensitivity to ischemic injury.

We find the average parenchymal volume of the whole brain to differ between the sexes, albeit non-significantly $(\mathrm{p}=0.065)$. This comparison revealed females to have on average a considerably greater volume of parenchyma than do males (Figure 5.3). In a study by Hammelrath (2016), reported ongoing brain development in mice at three months of age, including cortical flattening and increased myelination (Hammelrath et al., 2016). The study demonstrated that the largest rate of myelination occurred by three months of age. Unfortunately, the sexes of the mice used in the study were not reported. In others published work, have demonstrated a wide discrepancy in the timing of brain development between female and male rodents (Mengler et al., 2014). It is for these reasons, that we predict that the differences we observed in the average parenchymal volume between female and male mice is likely caused by temporal differences in brain development between the sexes. Specifically, 
female brain development may be temporally ahead of the development in males and since brain volume continues to increase until around 2 months of age and myelination at around 3 months of age. Our measured parenchymal volume in mice of both sexes may have captured two different stages of brain development.

The majority of the literature reporting the cerebral angioarchitecture employs paradigms that utilize fluorescent dyes (Blinder et al., 2013; Lugo-Hernandez et al., 2017; Zhang et al., 2018), chromatic dyes (Hasan, Herz, Hermann, \& Doeppner, 2013; Xiong et al., 2017; Xue et al., 2014), radio opaque solutions (Walker, Shen, Young, \& Su, 2011), and antibodies targeted against vascular epitopes (Park et al., 2014). The unifying limitations of these methods are inadequate imaging depth and the tradeoff between resolution and field of view. Light sheet microscopy (LSM) has become a popular platform for imaging large samples. However, because LSM is an optical imaging method, the quality of the images obtained is dependent on the fluorescence and transparency of the specimen. Mounting of specimens for LSM is also problematic, to prevent shrinkage caused by drying, the specimen must be either embedded or submerged without compromising the transparency of the specimen or increasing signal-noise (Santi, 2011; Watkins \& St Croix, 2018). Similar to other fluorescence based imaging platforms, LSM is prone to image aberrations, blurring, scaling by refractive index, autofluorescence, and signal degradation that progressively get worst with acquisition time and imaging depth (Santi, 2011; Watkins \& St Croix, 2018). To date, the aforementioned techniques have demonstrated difficulty imaging the complete angioarchitecture of the entire brain. Reported in the literature indicate that investigators are encountering complications of achieving perfusion of capillaries (Ghanavati, Yu, Lerch, \& Sled, 2014; Lugo-Hernandez et al., 2017; Pathak, Kim, Zhang, \& Jones, 2011; Walker et al., 
2011), observing broken vessel segments (Amato, Pan, Schwartz, \& Ragan, 2016; LugoHernandez et al., 2017; Pathak et al., 2011; Walker et al., 2011), and incomplete vascular networks (Amato et al., 2016; Ghanavati et al., 2014; Lugo-Hernandez et al., 2017).

A growing concern to all fields of research is our current technological limitations. Large data sets are difficult to post-process and even more so to perform three-dimensional quantitation and other complex algorithms for vascular network mapping and predictive simulations. We describe a procedure for imaging the entire brain vasculature, producing a single data set gigabytes in size.

We report a procedure to acquire volumetric data of the cerebrovasculature for threedimensional reconstruction and analysis of the whole brain in adult mice. Our analysis of the whole brain reveled sex-related differences in vascular topology. These differences were observed over the whole brain and varied by brain subregion. Overall, our study describes for the first time, a whole brain analysis of the cerebrovascular network and provides fundamental information for the advancement of vascular research.

\section{Acknowledgments}

This work was supported by NIH grants P20 GM109098, P01 AG027956, T32 AG052375, K01 NS081014 and U54 GM104942. Imaging experiments and image analysis were performed in the West Virginia University Microscope Imaging Facility, which has been supported by the Mary Babb Randolph Cancer Center and NIH grants P20 RR016440, P30 RR032138/GM103488 and P20 RR016477. DDQ designed studies, conducted studies and composed the manuscript. SEL, YA, JAG, SNS, JZC, and CMB aided with studies, analyzed 
data, and revised the manuscript. JWS designed studies and revised the manuscript. The authors declare no competing financial interest.

\section{References}

Aanerud, J., Borghammer, P., Rodell, A., Jónsdottir, K. Y., \& Gjedde, A. (2017). Sex differences of human cortical blood flow and energy metabolism. Journal of Cerebral Blood Flow \& Metabolism, 37(7), 2433-2440. https://doi.org/10.1177/0271678X16668536

Abbott, N. J., Patabendige, A. A. K., Dolman, D. E. M., Yusof, S. R., \& Begley, D. J. (2010). Structure and function of the blood-brain barrier. Neurobiology of Disease, 37(1), 13-25. https://doi.org/10.1016/j.nbd.2009.07.030

Alkayed, N. J., Harukuni, I., Kimes, A. S., London, E. D., Traystman, R. J., \& Hurn, P. D. (1998). Gender-linked brain injury in experimental stroke. Stroke, 29(1), 159-165; discussion 166. Retrieved from http://www.ncbi.nlm.nih.gov/pubmed/9445346

Alonso-Nanclares, L., Gonzalez-Soriano, J., Rodriguez, J. R., \& DeFelipe, J. (2008). Gender differences in human cortical synaptic density. Proceedings of the National Academy of Sciences, 105(38), 14615-14619. https://doi.org/10.1073/pnas.0803652105

Amato, S. P., Pan, F., Schwartz, J., \& Ragan, T. M. (2016). Whole Brain Imaging with Serial Two-Photon Tomography. Frontiers in Neuroanatomy, 10, 31. https://doi.org/10.3389/fnana.2016.00031

Amen, D. G., Trujillo, M., Keator, D., Taylor, D. V., Willeumier, K., Meysami, S., \& Raji, C. A. (2017). Gender-Based Cerebral Perfusion Differences in 46,034 Functional Neuroimaging Scans. Journal of Alzheimer's Disease, 60(2), 605-614. https://doi.org/10.3233/JAD-170432

Attwell, D., \& Laughlin, S. B. (2001). An energy budget for signaling in the grey matter of the brain. Journal of Cerebral Blood Flow and Metabolism: Official Journal of the International Society of Cerebral Blood Flow and Metabolism, 21(10), 1133-1145. 
https://doi.org/10.1097/00004647-200110000-00001

Beckmann, N., Schuler, A., Mueggler, T., Meyer, E. P., Wiederhold, K.-H., Staufenbiel, M., \& Krucker, T. (2003). Age-dependent cerebrovascular abnormalities and blood flow disturbances in APP23 mice modeling Alzheimer's disease. The Journal of Neuroscience : The Official Journal of the Society for Neuroscience, 23(24), 8453-8459. Retrieved from http://www.ncbi.nlm.nih.gov/pubmed/13679413

Blinder, P., Tsai, P. S., Kaufhold, J. P., Knutsen, P. M., Suhl, H., \& Kleinfeld, D. (2013). The cortical angiome: an interconnected vascular network with noncolumnar patterns of blood flow. Nature Neuroscience, 16(7), 889-897. https://doi.org/10.1038/nn.3426

Cassot, F., Lauwers, F., Fouard, C., Prohaska, S., \& Lauwers-Cances, V. (2006). A novel threedimensional computer-assisted method for a quantitative study of microvascular networks of the human cerebral cortex. Microcirculation (New York, N.Y.: 1994), 13(1), 1-18. https://doi.org/10.1080/10739680500383407

Cassot, F., Lauwers, F., Lorthois, S., Puwanarajah, P., Cances-Lauwers, V., \& Duvernoy, H. (2010). Branching patterns for arterioles and venules of the human cerebral cortex. Brain Research, 1313, 62-78. https://doi.org/10.1016/j.brainres.2009.12.007

Cassot, F., Lauwers, F., Lorthois, S., Puwanarajah, P., \& Duvernoy, H. (2009). Scaling Laws for Branching Vessels of Human Cerebral Cortex. Microcirculation, 16(4), 331-344. https://doi.org/10.1080/10739680802662607

Corliss, B. A., Mathews, C., Doty, R., Rohde, G., \& Peirce, S. M. (2018). Methods to label, image, and analyze the complex structural architectures of microvascular networks. Microcirculation (New York, N.Y. : 1994), e12520. https://doi.org/10.1111/micc.12520

Demené, C., Tiran, E., Sieu, L.-A., Bergel, A., Gennisson, J. L., Pernot, M., ... Tanter, M. (2016). 4D microvascular imaging based on ultrafast Doppler tomography. NeuroImage, 127, 472-483. https://doi.org/10.1016/j.neuroimage.2015.11.014

Douglas, R. J., \& Martin, K. A. C. (2004). Neuronal circuits of the neocortex. Annual Review of Neuroscience, 27(1), 419-451. https://doi.org/10.1146/annurev.neuro.27.070203.144152

Faber, J. E., Moore, S. M., Lucitti, J. L., Aghajanian, A., \& Zhang, H. (2017). Sex Differences 
in the Cerebral Collateral Circulation. Translational Stroke Research, 8(3), 273-283. https://doi.org/10.1007/s12975-016-0508-0

Fukuda, M., Moon, C.-H., Wang, P., \& Kim, S.-G. (2006). Mapping iso-orientation columns by contrast agent-enhanced functional magnetic resonance imaging: reproducibility, specificity, and evaluation by optical imaging of intrinsic signal. The Journal of Neuroscience: The Official Journal of the Society for Neuroscience, 26(46), 1182111832. https://doi.org/10.1523/JNEUROSCI.3098-06.2006

Ghanavati, S., Yu, L. X., Lerch, J. P., \& Sled, J. G. (2014). A perfusion procedure for imaging of the mouse cerebral vasculature by X-ray micro-CT. Journal of Neuroscience Methods, 221, 70-77. https://doi.org/10.1016/j.jneumeth.2013.09.002

Gould, I. G., Tsai, P., Kleinfeld, D., \& Linninger, A. (2017). The capillary bed offers the largest hemodynamic resistance to the cortical blood supply. Journal of Cerebral Blood Flow \& Metabolism, 37(1), 52-68. https://doi.org/10.1177/0271678X16671146

Gross, L. (2006). Redundancy in cortical surface vessels supports persistent blood flow. PLoS Biology, 4(2), e43. https://doi.org/10.1371/journal.pbio.0040043

Guibert, R., Fonta, C., \& Plouraboué, F. (2010). Cerebral Blood Flow Modeling in Primate Cortex. Journal of Cerebral Blood Flow \& Metabolism, 30(11), 1860-1873. https://doi.org/10.1038/jcbfm.2010.105

Hammelrath, L., Škokić, S., Khmelinskii, A., Hess, A., van der Knaap, N., Staring, M., ... Hoehn, M. (2016). Morphological maturation of the mouse brain: An in vivo MRI and histology investigation. NeuroImage, 125, 144-152. https://doi.org/10.1016/J.NEUROIMAGE.2015.10.009

Harel, N., Lin, J., Moeller, S., Ugurbil, K., \& Yacoub, E. (2006). Combined imaginghistological study of cortical laminar specificity of fMRI signals. NeuroImage, 29(3), 879-887. https://doi.org/10.1016/j.neuroimage.2005.08.016

Harrison, R. V, Harel, N., Panesar, J., \& Mount, R. J. (2002). Blood capillary distribution correlates with hemodynamic-based functional imaging in cerebral cortex. Cerebral Cortex (New York, N.Y.: 1991), 12(3), 225-233. Retrieved from http://www.ncbi.nlm.nih.gov/pubmed/11839597 
Hartung, G., Vesel, C., Morley, R., Alaraj, A., Sled, J., Kleinfeld, D., \& Linninger, A. (2018). Simulations of blood as a suspension predicts a depth dependent hematocrit in the circulation throughout the cerebral cortex. PLoS Computational Biology, 14(11), e1006549. https://doi.org/10.1371/journal.pcbi.1006549

Hasan, M. R., Herz, J., Hermann, D. M., \& Doeppner, T. R. (2013). Intravascular perfusion of carbon black ink allows reliable visualization of cerebral vessels. Journal of Visualized Experiments : JoVE, (71). https://doi.org/10.3791/4374

Heinzer, S., Krucker, T., Stampanoni, M., Abela, R., Meyer, E. P., Schuler, A., ... Müller, R. (2006). Hierarchical microimaging for multiscale analysis of large vascular networks. NeuroImage, 32(2), 626-636. https://doi.org/10.1016/j.neuroimage.2006.03.043

Hirsch, S., Reichold, J., Schneider, M., Székely, G., \& Weber, B. (2012). Topology and Hemodynamics of the Cortical Cerebrovascular System. Journal of Cerebral Blood Flow \& Metabolism, 32(6), 952-967. https://doi.org/10.1038/jcbfm.2012.39

Hodde, K. C., \& Nowell, J. A. (1980). SEM of micro-corrosion casts. Scanning Electron Microscopy, ( $\mathrm{Pt} \quad$ 2), 89-106. from http://www.ncbi.nlm.nih.gov/pubmed/6999611

Kennel, P., Teyssedre, L., Colombelli, J., \& Plouraboué, F. (2018). Toward quantitative threedimensional microvascular networks segmentation with multiview light-sheet fluorescence microscopy. Journal of Biomedical Optics, 23(08), 1. https://doi.org/10.1117/1.JBO.23.8.086002

Krucker, T., Lang, A., \& Meyer, E. P. (2006). New polyurethane-based material for vascular corrosion casting with improved physical and imaging characteristics. Microscopy Research and Technique, 69(2), 138-147. https://doi.org/10.1002/jemt.20263

Krucker, T., Schuler, A., Meyer, E. P., Staufenbiel, M., \& Beckmann, N. (2004). Magnetic resonance angiography and vascular corrosion casting as tools in biomedical research: application to transgenic mice modeling Alzheimer's disease. Neurological Research, 26(5), 507-516. https://doi.org/10.1179/016164104225016281

Lauwers, F., Cassot, F., Lauwers-Cances, V., Puwanarajah, P., \& Duvernoy, H. (2008). Morphometry of the human cerebral cortex microcirculation: general characteristics and 
space-related $\quad$ profiles. $\quad$ NeuroImage, 39(3), 936-948. https://doi.org/10.1016/j.neuroimage.2007.09.024

Lawal, A., Kern, M., Sanjeevi, A., Hofmann, C., \& Shaker, R. (2005). Cingulate cortex: a closer look at its gut-related functional topography. https://doi.org/10.1152/ajpgi.00016.2005.-Earlier

Lein, E. S., Hawrylycz, M. J., Ao, N., Ayres, M., Bensinger, A., Bernard, A., ... Jones, A. R. (2007). Genome-wide atlas of gene expression in the adult mouse brain. Nature, 445(7124), 168-176. https://doi.org/10.1038/nature05453

Li, Y., \& Chi, I. (2011). Correlates of physician visits among older adults in China: the effects of family support. Journal of Aging and Health, 23(6), 933-953. https://doi.org/10.1177/0898264311401390

Liu, J., Zubieta, J.-K., \& Heitzeg, M. (2012). Sex differences in anterior cingulate cortex activation during impulse inhibition and behavioral correlates. Psychiatry Research, 201(1), 54-62. https://doi.org/10.1016/j.pscychresns.2011.05.008

Lorthois, S., \& Cassot, F. (2010). Fractal analysis of vascular networks: insights from morphogenesis. Journal of Theoretical Biology, 262(4), 614-633. https://doi.org/10.1016/j.jtbi.2009.10.037

Lugo-Hernandez, E., Squire, A., Hagemann, N., Brenzel, A., Sardari, M., Schlechter, J., ... Hermann, D. M. (2017). 3D visualization and quantification of microvessels in the whole ischemic mouse brain using solvent-based clearing and light sheet microscopy. Journal of Cerebral Blood Flow and Metabolism : Official Journal of the International Society of Cerebral Blood Flow and Metabolism, 37(10), 3355-3367. https://doi.org/10.1177/0271678X17698970

Lyons, D. G., Parpaleix, A., Roche, M., \& Charpak, S. (2016). Mapping oxygen concentration in the awake mouse brain. ELife, 5. https://doi.org/10.7554/eLife.12024

Magistretti, P. J., \& Pellerin, L. (1999). Cellular mechanisms of brain energy metabolism and their relevance to functional brain imaging. Philosophical Transactions of the Royal Society of London. Series B, Biological Sciences, 354(1387), 1155-1163. https://doi.org/10.1098/rstb.1999.0471 
Manwani, B., Bentivegna, K., Benashski, S. E., Venna, V. R., Xu, Y., Arnold, A. P., \& McCullough, L. D. (2015). Sex differences in ischemic stroke sensitivity are influenced by gonadal hormones, not by sex chromosome complement. Journal of Cerebral Blood Flow and Metabolism: Official Journal of the International Society of Cerebral Blood Flow and Metabolism, 35(2), 221-229. https://doi.org/10.1038/jcbfm.2014.186

Mengler, L., Khmelinskii, A., Diedenhofen, M., Po, C., Staring, M., Lelieveldt, B. P. F., \& Hoehn, M. (2014). Brain maturation of the adolescent rat cortex and striatum: Changes in volume and myelination. Neurolmage, 35-44. https://doi.org/10.1016/j.neuroimage.2013.08.034

Meyer, E. P., Ulmann-Schuler, A., Staufenbiel, M., \& Krucker, T. (2008). Altered morphology and 3D architecture of brain vasculature in a mouse model for Alzheimer's disease. Proceedings of the National Academy of Sciences of the United States of America, 105(9), 3587-3592. https://doi.org/10.1073/pnas.0709788105

Moore, S. M., Zhang, H., Maeda, N., Doerschuk, C. M., \& Faber, J. E. (2015). Cardiovascular risk factors cause premature rarefaction of the collateral circulation and greater ischemic tissue injury. Angiogenesis, 18(3), 265-281. https://doi.org/10.1007/s10456-015-9465-6

Nyengaard, J. R. (1999). Stereologic methods and their application in kidney research. Journal of the American Society of Nephrology: JASN, 10(5), 1100-1123. Retrieved from http://www.ncbi.nlm.nih.gov/pubmed/10232698

Pardridge, W. M. (2007). Blood-brain barrier delivery. Drug Discovery Today, 12(1-2), 5461. https://doi.org/10.1016/j.drudis.2006.10.013

Park, L., Koizumi, K., El Jamal, S., Zhou, P., Previti, M. Lou, Van Nostrand, W. E., ... Iadecola, C. (2014). Age-dependent neurovascular dysfunction and damage in a mouse model of cerebral amyloid angiopathy. Stroke, 45(6), 1815-1821. https://doi.org/10.1161/STROKEAHA.114.005179

Pathak, A. P., Kim, E., Zhang, J., \& Jones, M. V. (2011). Three-dimensional imaging of the mouse neurovasculature with magnetic resonance microscopy. PloS One, 6(7), e22643. https://doi.org/10.1371/journal.pone.0022643

Peyrounette, M., Davit, Y., Quintard, M., \& Lorthois, S. (2018). Multiscale modelling of blood 
flow in cerebral microcirculation: Details at capillary scale control accuracy at the level of the cortex. PloS One, 13(1), e0189474. https://doi.org/10.1371/journal.pone.0189474

Pushpavalli, R. ., \& Sivarajde, G. (2013). A Hybrid Filtering Technique for Random Valued Impulse Noise Elimination on Digital Images. Retrieved from https://www.semanticscholar.org/paper/A-Hybrid-Filtering-Technique-for-RandomValued-on-Pushpavalli-Sivarajde/2363dbd4a7dbcb7ff19e0ca10fde7d40b24659a2

Ridler, T. ., \& Calvard, S. (1978). Picture Thresholding Using an Iterative Selection Method. IEEE Transactions on Systems, Man, and Cybernetics, 8(8), 630-632. https://doi.org/10.1109/TSMC.1978.4310039

Santi, P. A. (2011). Light sheet fluorescence microscopy: a review. The Journal of Histochemistry and Cytochemistry: Official Journal of the Histochemistry Society, 59(2), 129-138. https://doi.org/10.1369/0022155410394857

Schmid, F., Tsai, P. S., Kleinfeld, D., Jenny, P., \& Weber, B. (2017). Depth-dependent flow and pressure characteristics in cortical microvascular networks. PLoS Computational Biology, 13(2), e1005392. https://doi.org/10.1371/journal.pcbi.1005392

Sheth, S. A., \& Liebeskind, D. S. (2014). \&quot;Imaging Evaluation of Collaterals in the Brain: Physiology and Clinical Translation\&quot; Current Radiology Reports, 2(1), 29. https://doi.org/10.1007/s40134-013-0029-5

Smith, A. F., Doyeux, V., Berg, M., Peyrounette, M., Haft-Javaherian, M., Larue, A.-E., ... Lorthois, S. (2019). Brain Capillary Networks Across Species: A few Simple Organizational Requirements Are Sufficient to Reproduce Both Structure and Function. Frontiers in Physiology, 10, 233. https://doi.org/10.3389/fphys.2019.00233

Starosolski, Z., Villamizar, C. A., Rendon, D., Paldino, M. J., Milewicz, D. M., Ghaghada, K. B., \& Annapragada, A. V. (2015). Ultra High-Resolution In vivo Computed Tomography Imaging of Mouse Cerebrovasculature Using a Long Circulating Blood Pool Contrast Agent. Scientific Reports, 5(1), 10178. https://doi.org/10.1038/srep10178

Tsai, P. S., Kaufhold, J. P., Blinder, P., Friedman, B., Drew, P. J., Karten, H. J., ... Kleinfeld, D. (2009). Correlations of neuronal and microvascular densities in murine cortex revealed by direct counting and colocalization of nuclei and vessels. The Journal of Neuroscience : 
The Official Journal of the Society for Neuroscience, 29(46), 14553-14570. https://doi.org/10.1523/JNEUROSCI.3287-09.2009

Tsai, W.-H. (1985). NOTE Moment-Preserving Thresholding: A New Approach. In VISION. GRAPHICS, AND IMAGE PROCESSING (Vol. 29). Retrieved from https://people.cs.nctu.edu.tw/ whtsai/Journal

Paper PDFs/Tsai_CVGIP(journal)_1985.pdf

Uğurbil, K., Adriany, G., Andersen, P., Chen, W., Garwood, M., Gruetter, R., ... Zhu, X.-H. (2003). Ultrahigh field magnetic resonance imaging and spectroscopy. Magnetic Resonance Imaging, 2l(10), 1263-1281. Retrieved from http://www.ncbi.nlm.nih.gov/pubmed/14725934

Walker, E. J., Shen, F., Young, W. L., \& Su, H. (2011). Cerebrovascular casting of the adult mouse for 3D imaging and morphological analysis. Journal of Visualized Experiments : JoVE, (57), e2958. https://doi.org/10.3791/2958

Watkins, S. C., \& St Croix, C. M. (2018). Light sheet imaging comes of age. The Journal of Cell Biology, 217(5), 1567-1569. https://doi.org/10.1083/jcb.201804016

Whitesall, S. E., Hoff, J. B., Vollmer, A. P., \& D’Alecy, L. G. (2004). Comparison of simultaneous measurement of mouse systolic arterial blood pressure by radiotelemetry and tail-cuff methods. American Journal of Physiology. Heart and Circulatory Physiology, 286(6), H2408-15. https://doi.org/10.1152/ajpheart.01089.2003

Wilde, E., Aubdool, A. A., Thakore, P., Baldissera, L., Alawi, K. M., Keeble, J., ... Brain, S. D. (2017). Tail-Cuff Technique and Its Influence on Central Blood Pressure in the Mouse. Journal of the American Heart Association, 6(6). https://doi.org/10.1161/JAHA.116.005204

Xiong, B., Li, A., Lou, Y., Chen, S., Long, B., Peng, J., ... Gong, H. (2017). Precise Cerebral Vascular Atlas in Stereotaxic Coordinates of Whole Mouse Brain. Frontiers in Neuroanatomy, 11, 128. https://doi.org/10.3389/fnana.2017.00128

Xue, S., Gong, H., Jiang, T., Luo, W., Meng, Y., Liu, Q., ... Li, A. (2014). Indian-ink perfusion based method for reconstructing continuous vascular networks in whole mouse brain. PloS One, 9(1), e88067. https://doi.org/10.1371/journal.pone.0088067 
Zhang, L.-Y., Lin, P., Pan, J., Ma, Y., Wei, Z., Jiang, L., ... Yang, G.-Y. (2018). CLARITY for High-resolution Imaging and Quantification of Vasculature in the Whole Mouse Brain. Aging and Disease, 9(2), 262-272. https://doi.org/10.14336/AD.2017.0613

Zhao, F., Wang, P., Hendrich, K., Ugurbil, K., \& Kim, S.-G. (2006). Cortical layer-dependent BOLD and CBV responses measured by spin-echo and gradient-echo fMRI: insights into hemodynamic regulation. NeuroImage, 30(4), 1149-1160. https://doi.org/10.1016/j.neuroimage.2005.11.013 
A
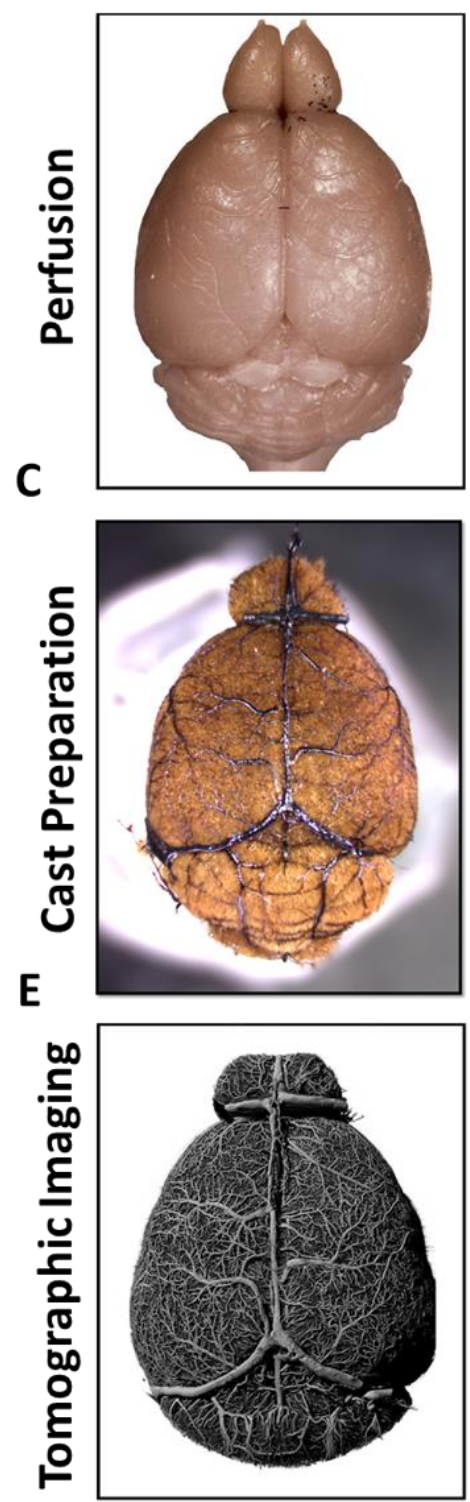

B
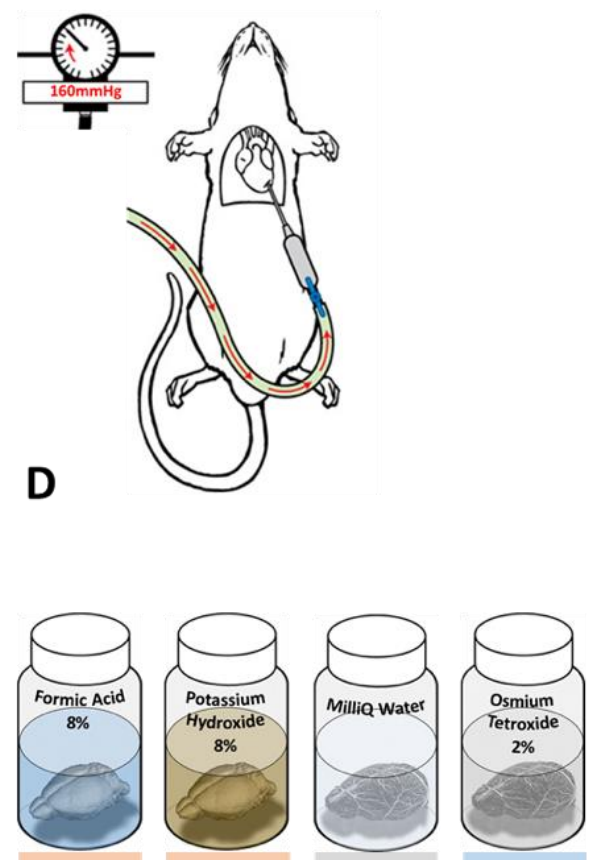

$37^{\circ} \mathrm{C}$

$37^{\circ} \mathrm{C}$

$25^{\circ} \mathrm{C}$

$4{ }^{\circ} \mathrm{C}$
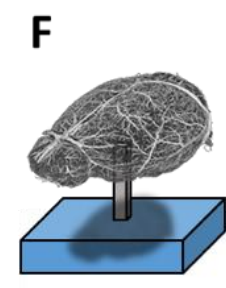

G

X-ray generator
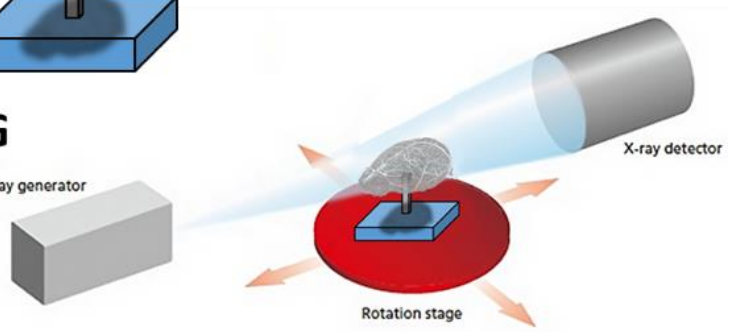

Supplemental Figure 5.1. Workflow of the procedure used to produce the cerebrovascular corrosion casts. (A) Perfusion of the adult mouse brain. (B) Transcardial perfusion via cannulated ascending aorta of the eluate, fixative, and casting resin at 160 mmHg. (C) Preprocessing of the vascular corrosion cast. (D) Decalcification, maceration, washing, and osmium embedding of the vascular corrosion casts. (E) Processing of the vascular corrosion cast for tomographic imaging. (F) Mounting and positioning of vascular cast on pedestals. (G) Setup of Image acquisition routine for $\mu$ computerized tomographic imaging of vascular corrosion cast. 

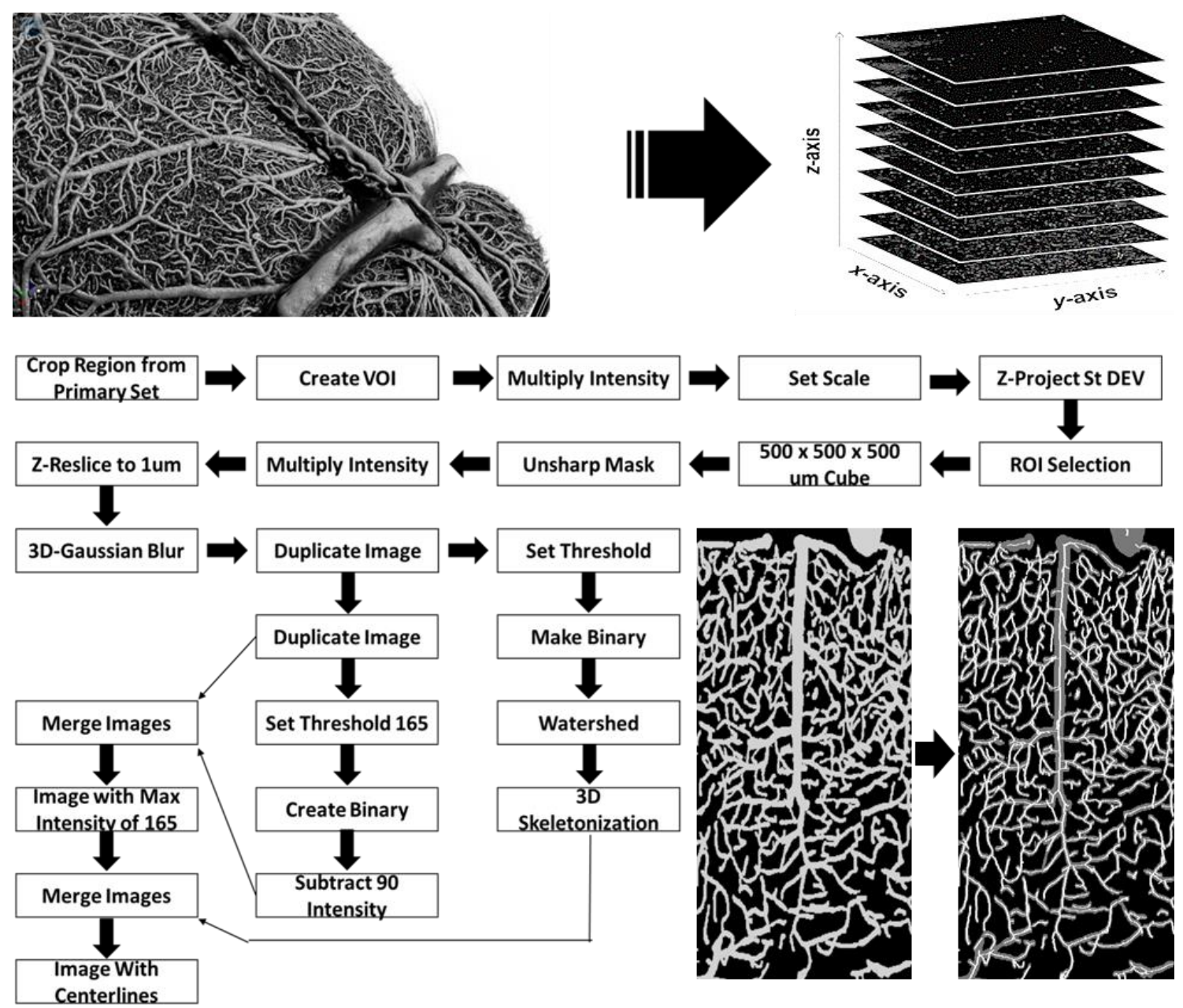

Supplemental Figure 5.2. Process flow diagram for brain vascular subregion optimization by segmentation and centerline insertion. Specific brain subregions were isolated into separate VOI image sets by 3-dimensional cropping of the primary data set. The intensity of each VOI data set was normalized to the average intensity of all VOIs by arithmetic adjustment. Standard deviation intensity projection of the image series was created to aid in the co-registration of the data set to a specified orientation. The final coregistered image series produced a $500 \times 500$ x 500 um VOI readjusted to a pixel size of 1um. The VOI data set was duplicated to produce three identical sub-blocks. The first subblock was converted into a binary image series then used to extract the centerlines of vessels. The second sub-block served as a mask to subtract the intensity of all regions above 165 in the third sub-block. Finally, the centerlines of the first sub-block is merged with the third sub-block producing an image series with centerlines having a pixel intensity of 255 and vascular structures with a pixel intensity $<165$. 


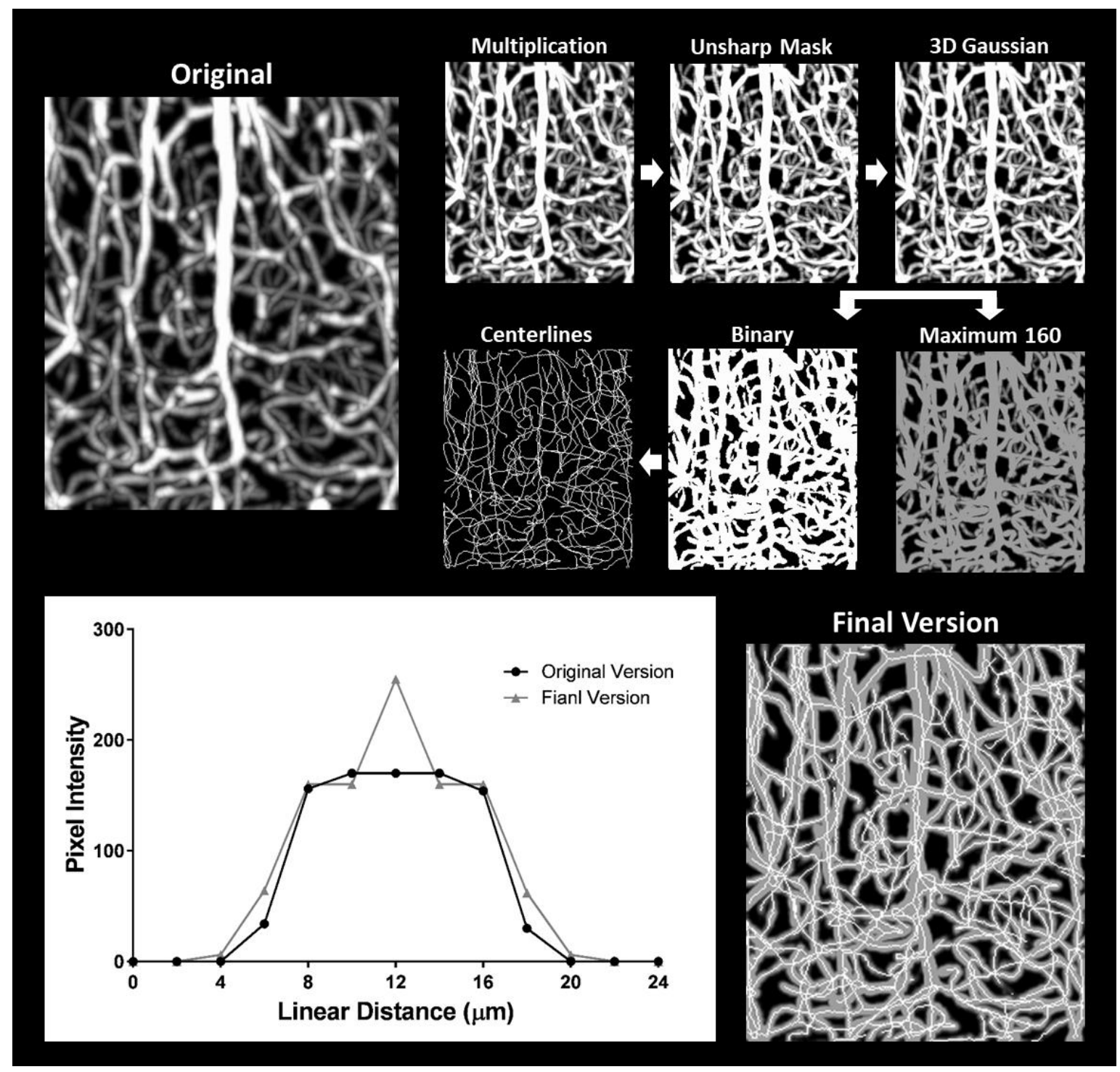

Supplemental Figure 5.3. The post-processing of image data sets causes no artifactual alterations to vessel diameter or destruction of the native network architecture. The maximum intensity projection of a data set depicting cortical vessels after post-processing of an image by multiplication, unsharp mask, 3D Gaussian filter, maximum pixel intensity cutoff, binarization, centerline extraction and final image composition. Line graph demonstrating the linear pixel intensity profile ( $y$-axis) across the entire diameter ( $x$-axis) of a vessel before (black circles) and after the post-processing (gray triangle) of image data. The final version of the data set contains centerlines at a maximum pixel intensity of 255 while non-centerline pixel intensities are cut off at a maximum of 160 . 

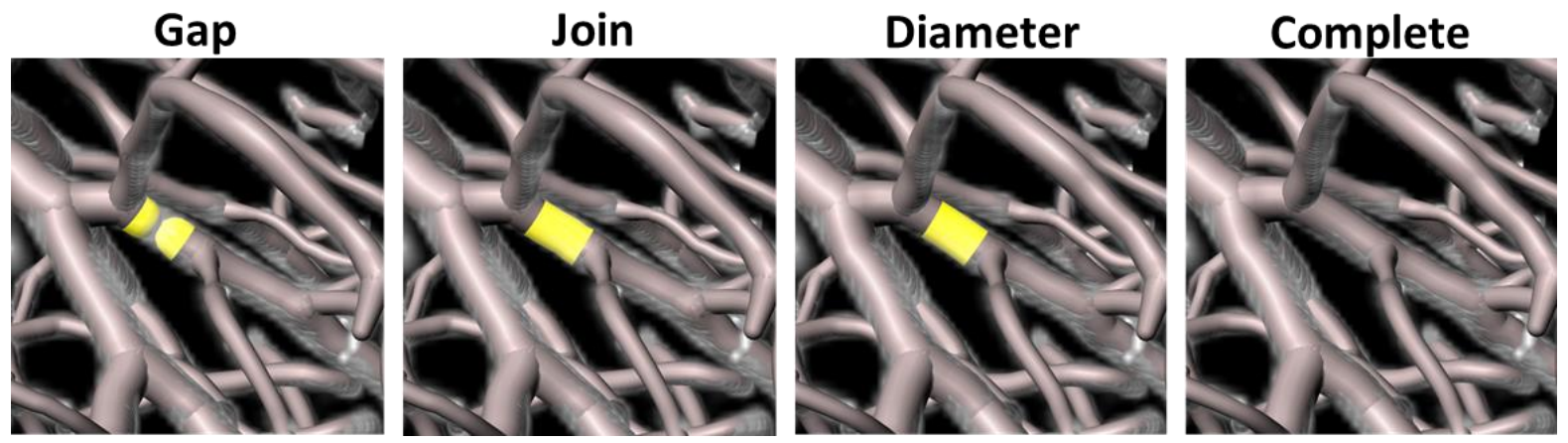

Supplemental Figure 5.4. Manual error corrections following an automated filament trace function. Depiction of an erroneous gap (first panel, yellow) after automated filament tracing resolved by manually joining (second panel) the segment together followed by recalculating the diameter of the new portion of segment (third panel, yellow) from the image data set. Final version of a vessel filament that was manually corrected for a gaperror (fourth panel) that appeared following the automated filament trace function. 

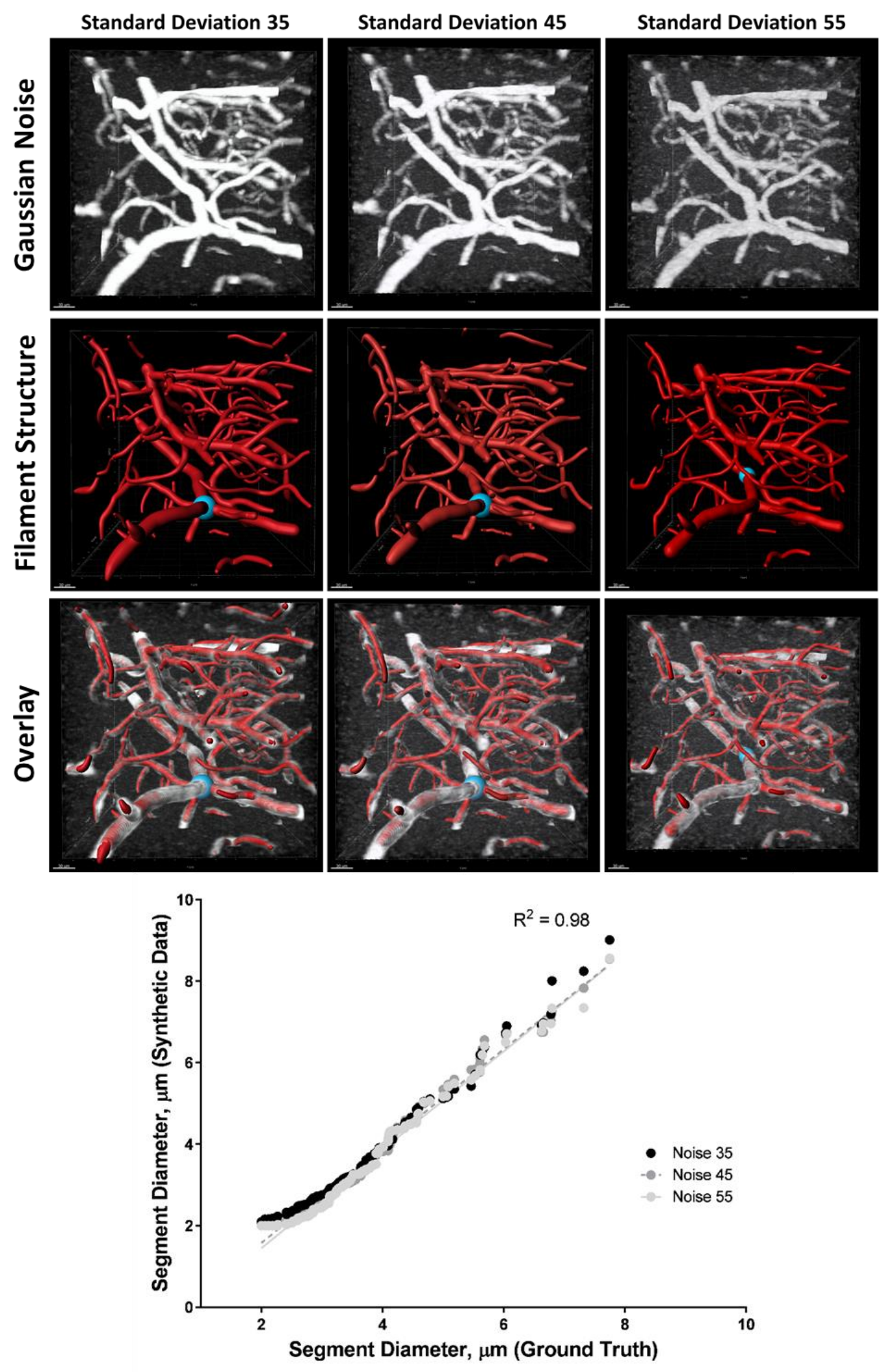


\section{Supplemental Figure 5.5. Effect of image noise on the three-dimensional vascular}

network reconstruction and analysis. Gaussian noise (top row) at a standard deviation of 35,45 , or 55 , was artificially introduced into a $250 \times 250 \times 250 \mu \mathrm{m}^{3}$ data set. The quality of the generated filament structures (middle row) from each of the data sets was inspected. The specific positioning of the reconstructed filaments for each data set can be seen by overlay (bottom row). (A) Scatter plot of the measured filament diameters from each data set containing elevated Gaussian noise as a function to the filament diameters of the manually segmented and manually traced data set. Linear trend indicates agreement between the measured filament diameters. $\mathrm{R}^{2}$ value indicates the strength of fit to the linear model. 
Image Post-Processing

\begin{tabular}{lcl}
\hline Function & Parameter(s) & \multicolumn{1}{c}{ Image Adjustment } \\
\hline Multiplication & Image $=$ Image $\mathrm{1.5}$ & Used to shift up the average intensity of the original data set for thresholding. \\
Gaussian Filter & Sigma $=0.65$ pixels & Convolution with Gaussian function. Smooths intensity of structures to aid in thre sholding for binarization. \\
Unsharp Mask & Sigma = 1 pixel / Mask Weight = 0.06 & Enhances local contrast to increase spatial resolvability. \\
Binarization & Moments Algorithm & To convert the 8-bit gray scale data set into a binary data set. \\
Centerline Extraction & 3D Extraction from Binary Image & Used to obtain the center most pixel along the vessel structure's length in 3D space. \\
Maximum (Image Math) & Pixel I = Pixel I- (Pixel I- 160) & No pixel above 160. Primes image for centerline insertion at an intensity of 255. Only centerlines at 255. \\
\hline
\end{tabular}

Supplemental Table 1. Image post-processing functions used to optimize data sets prior to analysis. Multiplication: global pixel arithmatic; Gaussian Filter: convolving filter; Unsharp Mask: sharpen filter; Binarization: image format transformation; Centerline Extraction: morphological function; Maximum: global pixel arithmatic. 


\begin{tabular}{|c|c|c|c|c|c|c|}
\hline & \multicolumn{2}{|c|}{ Female } & \multicolumn{2}{|c|}{ Male } & \multirow[b]{2}{*}{ P-value } & \multirow[b]{2}{*}{ Units } \\
\hline & Value & SEM & Value & SEM & & \\
\hline Age & 3.0 & 0 & 3.0 & 0 & $n \cdot r$ & Months \\
\hline Weight & 30 & 0.5 & 30 & 0.5 & 0.489 & Grams \\
\hline Temperature & 36 & 0.1 & 36 & 0.1 & 0.125 & $\mathrm{C}^{\circ}$ \\
\hline Systolic Blood Pressure & 164 & 0.1 & 165 & 0.1 & 0.004 & $\mathrm{mmHg}$ \\
\hline Diastolic Blood Pressure & 142 & 0.2 & 140 & 0.4 & 0.027 & $\mathrm{mmHg}$ \\
\hline Mean Arterial Pressure & 149 & 0.2 & 148 & 0.3 & 0.057 & $\mathrm{mmHg}$ \\
\hline \multicolumn{7}{|c|}{ Whold Brain Analysis } \\
\hline Vessel Diameter Range & $2-220$ & - & $2-220$ & - & n.r. & $\mu \mathrm{m}$ \\
\hline Mean Diameter & 36.6 & 2.4 & 54.3 & 10.6 & 0.095 & $\mu \mathrm{m}$ \\
\hline Number of Vessels & $1.4 \times 10^{6}$ & $2.6 \times 10^{5}$ & $1.8 \times 10^{6}$ & $3.0 \times 10^{5}$ & 0.154 & Segments \\
\hline Intervessel Distance & 20 & 5 & 32 & 13 & 0.037 & $\mu \mathrm{m}$ \\
\hline Total Vascular Volume & $2.2 \times 10^{10}$ & $4.0 \times 10^{9}$ & $1.3 \times 10^{10}$ & $1.7 \times 10^{9}$ & 0.053 & $\mu \mathrm{m}^{3}$ \\
\hline Total Parencymal Volume & $1.5 \times 10^{11}$ & $5.1 \times 10^{10}$ & $4.7 \times 10^{10}$ & $1.7 \times 10^{10}$ & 0.065 & $\mu \mathrm{m}^{3}$ \\
\hline Parencymal Volume (\% Brain Volume) & 76.7 & 6.6 & 62.3 & 8.7 & 0.111 & $\%$ \\
\hline Vascular Surface Area & $5.5 \times 10^{9}$ & $1.1 \times 10^{9}$ & $3.2 \times 10^{9}$ & $6.2 \times 10^{8}$ & 0.075 & $\mu m^{2}$ \\
\hline Vessel Surface Area / Vessel Volume & 0.24 & 0.01 & 0.23 & 0.01 & 0.368 & $1 / \mu \mathrm{m}$ \\
\hline Vessel Population $<15 \mu \mathrm{m}$ & 50 & 6 & 40 & 3 & 0.084 & $\%$ of Total \\
\hline Vessel Population $20-80 \mu \mathrm{m}$ & 36.3 & 3 & 49 & 3 & 0.01 & $\%$ of Total \\
\hline BBB Interface $\left(S_{c} / V_{p}\right)$ & 17.8 & 3.2 & 17.8 & 3.2 & n.r. & $\mathrm{mm}^{2} / \mathrm{mm}^{3}$ \\
\hline Euler Number & $1.1 \times 10^{6}$ & $3.7 \times 10^{5}$ & $1.7 \times 10^{6}$ & $2.4 \times 10^{5}$ & 0.11 & Connections \\
\hline Connectivity Value (Redundancy) & $5.2 \times 10^{5}$ & $2.2 \times 10^{5}$ & $1.2 \times 10^{5}$ & $6.2 \times 10^{4}$ & 0.088 & Cuts \\
\hline Connective Density & $4.2 \times 10^{-5}$ & $8.0 \times 10^{-6}$ & $3.7 \times 10^{-5}$ & $4.7 \times 10^{-6}$ & 0.333 & $1 / \mu \mathrm{m}^{3}$ \\
\hline Fractal Dimension & 2.6 & 0.05 & 2.5 & 0.06 & 0.052 & \\
\hline \multicolumn{7}{|c|}{ Region Specific Analysis (Primary Somatosensory Cortex) } \\
\hline Number of Vessels & 660 & 54 & 468 & 107 & 0.071 & Segments \\
\hline Mean Diameter & 14.76 & 0.22 & 14.37 & 0.31 & 0.175 & $\mu \mathrm{m}$ \\
\hline Vascular Surface Area & $5.3 \times 10^{6}$ & $5.5 \times 10^{5}$ & $4.5 \times 10^{6}$ & $3.3 \times 10^{5}$ & 0.154 & $\mu \mathrm{m}^{2}$ \\
\hline Total Vascular Volume & $1.7 \times 10^{7}$ & $2.1 \times 10^{6}$ & $1.4 \times 10^{7}$ & $1.4 \times 10^{6}$ & 0.164 & $\mu \mathrm{m}^{3}$ \\
\hline Total Parencymal Volume & $1.0 \times 10^{8}$ & $2.1 \times 10^{6}$ & $1.1 \times 10^{8}$ & $1.4 \times 10^{6}$ & 0.164 & $\mu \mathrm{m}^{3}$ \\
\hline Intervessel Distance & 38.6 & 2.4 & 43.1 & 1.5 & 0.1 & $\mu \mathrm{m}$ \\
\hline Volume of Vessels $15 \mu \mathrm{m}$ & $6.5 \times 10^{6}$ & $1.2 \times 10^{6}$ & $3.8 \times 10^{6}$ & $7.7 \times 10^{5}$ & 0.01 & $\mu \mathrm{m}^{3}$ \\
\hline Parencymal Zones < $15 \mu \mathrm{m}$ & $3.2 \times 10^{4}$ & $6.5 \times 10^{3}$ & $2.4 \times 10^{4}$ & $2.7 \times 10^{3}$ & n.r. & Count \\
\hline Parencymal Zones $>15 \mu \mathrm{m}$ & $3.6 \times 10^{5}$ & $3.0 \times 10^{4}$ & $2.5 \times 10^{5}$ & $3.6 \times 10^{4}$ & 0.04 & Count \\
\hline Network Composition, Vessels $6 \mu \mathrm{m}$ & 1835 & 490 & 1082 & 102 & 0.01 & Segments \\
\hline Euler Number & 361 & 67 & 226 & 142 & 0.18 & Connections \\
\hline Connectivity Volue (Redundancy) & 587 & 134 & 366 & 95 & 0.14 & Cuts \\
\hline
\end{tabular}

Supplemental Table 2. Analysis of the whole brain cerebrovasculature and in the somatosensory cortex in female and male mice. Not reported $=$ n.r.. 


\section{CHAPTER 6}

\section{Microvascular Degeneration Occurs Before Plaque Onset and Progresses with Age in 3xTg AD Mice}

Dominic D. Quintana, Yamini Anantula, Jorge A. Garcia, Elizabeth B. Engler-Chiurazzi, Saumyendra N. Sarkar, Deborah R. Corbin, Candice M. Brown and James W. Simpkins

Department of Neuroscience

Center of Basic and Translational Stroke Research

Rockefeller Neuroscience Institute

West Virginia University, Morgantown, West Virginia, 26506

\section{Author Contribution Statement}

DDQ designed studies, conducted studies and composed the manuscript. YA, JAG and DRC conducted studies. SNS, EBEC and CMB revised the manuscript. JWS designed studies and revised the manuscript. 


\begin{abstract}
Cardiovascular disease and its corresponding risk factors are positively correlated with the incidence of $\mathrm{AD}$. Cerebrovascular hypoperfusion and microvascular dysfunction are common comorbidities in patients with Alzheimer's disease (AD). Although there is ostensible involvement of dysfunctional cerebrovasculature in AD pathophysiology, the characterization of the specific changes, and the time-course of the development of vascular injury during AD remains unclear. In the present study, we establish a time-course for the structural changes and degeneration of the angioarchitecture with the age-related progression of AD. We used cerebrovascular corrosion cast and $\mu \mathrm{CT}$ imaging to evaluate the geometry, topology, and complexity of the angioarchitecture in the brain of wild type and triple transgenic AD mice. We hypothesized that changes to the microvasculature could be identified during the early stages of the disease progression. We predicted that these early identifiable changes to the microvasculature would be more prominent in particular subregions of the brain that are implicated in the cognitive decline of AD. We found age- and disease-related deficits of cerebral blood flow in $3 \times \mathrm{Tg}$ AD mice. The whole-brain analysis of the angioarchitecture indicated early structural abnormalities and degeneration of microvascular networks in $3 \times \mathrm{Tg}$ AD. Our analysis of hippocampal and cortical subregions revealed microvascular degeneration with onset and progression that was subregion dependent.
\end{abstract}




\section{Significance Statement}

Cerebrovascular hypoperfusion and hypometabolism are fundamental neuropathological features of Alzheimer's disease. This study demonstrates in a mouse model of $\mathrm{AD}$, age-related changes to the angioarchitecture over the whole-brain that occurred early, before the onset of $A \beta$-plaque deposition and neurofibrillary tangles. Changes to the cerebrovasculature dramatically affected the microvasculature over the whole-brain and in key brain regions that are implicated in behavioral and cognitive deficits in AD. These results revealed a critical period of cerebrovascular dysfunction and degeneration during early presymptomatic stages of $\mathrm{AD}$ development. These findings provide a basis for development of novel therapeutic strategies targeting early cerebrovascular changes and may provide a prophylactic strategy for $\mathrm{AD}$ and indicate novel early biomarkers of the vasculature for diagnosis of the disease. 


\section{Introduction}

Alzheimer's disease (AD) is the most common form of dementia and is characterized by progressive neurodegeneration and cognitive decline. Histopathological examination of the postmortem brain from $A D$ patients demonstrates parenchymal deposition of amyloid- $\beta$ (A $\beta$ )plaques and neurofibrillary tangles formed by hyper-phosphorylated tau, both of which are neuropathological hallmarks of AD (Querfurth and LaFerla 2010; Castellani et al. 2010). The contribution of $\mathrm{A} \beta$ deposition to the development and progression of $\mathrm{AD}$ has been a central focus of $\mathrm{AD}$ research. Unfortunately, though many novel drugs targeting $\mathrm{A} \beta$ have entered clinical trials, none have been successful at altering the trajectory of the disease.

Comorbidities of the cerebrovasculature are frequently reported in $\mathrm{AD}$, as many as $92 \%$ of patients diagnosed with $\mathrm{AD}$ also demonstrate ischemic lesions of the white matter that resemble arteriosclerosis of small vessels (Rosenberg et al. 2016). It is well established that regional hypoperfusion, hypometabolism, and blood-brain barrier disruption are common pathological manifestations in patients with AD (de la Torre 2004; Brundel et al. 2012a; Brundel et al. 2012b). A $\beta$ is a potent vasoconstrictor (Thomas et al. 1996) and its production has been associated with impaired endothelium-dependent regulation of cortical microcirculation and aberrant functional hyperemia (Iadecola et al. 1999; Niwa et al. 2000). In rodents, transient exposure to brain ischemia results in increased neuronal tau, APP expression, and deposition of $\mathrm{A} \beta$ in the hippocampus and cortex (Iadecola 2004; Bailey et al. 2004). In addition, patients diagnosed with $\mathrm{AD}$ are at an increased risk for developing hemorrhagic stroke, cerebral microinfarctions, spontaneous cerebral emboli, and microhemorrhages (Brundel et al. 2012a; Chi et al. 2013; Purandare and Burns 2009; Tolppanen et al. 2013). Evidence from both clinical and animal studies has demonstrated that 
cerebrovascular impairment precedes the onset of neurodegeneration and progresses with agerelated cognitive decline and with dementia (de la Torre 2010; Jellinger 2010; Kalaria 2010; Fischer et al. 1990; Kalaria and Hedera 1995), suggesting that vascular dysfunction to participate in a causative manner in the emergence of $\mathrm{AD}$.

Remarkably, $90 \%$ of patients with $\mathrm{AD}$ demonstrate cerebral amyloid angiopathy (CAA), a neuropathological disease characterized by the deposition of amyloid- $\beta$ on the walls of cerebral vasculature (Janson 2015; DeSimone et al. 2017; Vinters 1987; Han et al. 2015). $\mathrm{A} \beta$ deposition on the basement membrane of blood vessels promotes local inflammation, and the activation of vascular endothelial cells, perivascular microglia, pericytes, and astrocytes (Bailey et al. 2004; Vinters and Farag 2003; Farkas et al. 2001), which contribute to the degradation of the vascular wall. In addition, $A \beta$ accumulation at blood vessels causes vascular smooth muscle cell (vSMC) degeneration (Van Nostrand et al. 2001), which together with the weakened blood vessel wall can increase the risk for hemorrhagic stroke that is common in patients with AD (Vinters and Farag 2003).

Despite demonstrations of strong evidence linking various pathology of the cerebrovasculature with $\mathrm{AD}$ etiology, the precise timeline and extent of cerebrovascular changes remain unclear, representing a critical barrier to research progress in the field. Data collected using an innovative whole-brain cerebrovascular casting approach demonstrated for the first time that $3 \times \mathrm{Tg} \mathrm{AD}$ mice display functionally significant disruptions in the cerebrovasculature that occur early in the disease time course and preceded the accumulation of $\mathrm{AD}$ neuropathological hallmarks such as $\mathrm{A} \beta$. 


\section{Materials and Methods}

\section{Animal Usage}

Procedures performed in this study that involved the use of laboratory animals were carried out in accordance with the National Institutes of Health guide for the care and use of laboratory animals (NIH Publication No. 8023, revised 1978) and in compliance with the ARRIVE guidelines. For the purposes of this study, we used the male B6; 129-Tg (APPSwe,

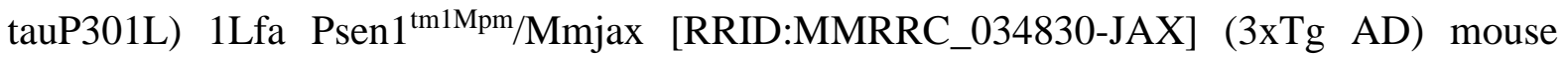
model of Alzheimer's disease and the B6129SF2/J (wild type, WT) mouse strains from the Mutant Mouse Resource and Research Center (MMRRC) at The Jackson Laboratory. To study the effects of aging, experiments were performed on these mice at 3 -months (WT $n=4,3 x T g$ AD n = 5), 6-months (WT n = 7, 3xTg AD n = 5), 12-months (WT n = 5, 3xTg AD n = 4), and 24-months (WT $n=5,3 x T g$ AD $n=4$ ) of age. Power analysis for ANOVA designs indicated a sample size of 4 mice per group (power $=0.999$ ) for an effect size of $\Delta=1.25$. Mice were maintained under a light/dark cycle $(12 \mathrm{~h}: 12 \mathrm{~h})$ with food and water available ad libitum at West Virginia University vivarium.

\section{Measurement of Cerebral Blood Flow}

Mice were deeply anesthetized with $4 \%$ isoflurane and maintained with $2 \%$ isoflurane diffused into a $30 \%$ oxygen and $70 \%$ nitrogen mixture. Mice were placed on a feedback controlled heating pad to maintain the body temperature at $37{ }^{\circ} \mathrm{C}$. Ophthalmic ointment was placed on each eye; then the surgical area was prepared by removing the fur and sanitizing the skin at the surgical site with isopropanol and betadine. A $1.0 \mathrm{~cm}$ incision was made on the 
scalp of each mouse. The skin was retracted to expose the skull and cleaned with a cotton swab. Ten consecutive measurements of cerebral blood flow (CBF) were acquired with a MoorFLPI laser speckle flowmeter (Moor Instruments, England) over the course of 5 minutes with an exposure of $200 \mathrm{~ms}$. After CBF measurements were acquired, the incision was closed by suturing (Quintana et al. 2018).

\section{Vascular Corrosion Casts}

For a detail description of the procedure used to prepare cerebrovascular corrosion casts, please refer to our previous report (Quintana et al. 2019). Briefly, mice were deeply anesthetized with $4 \%$ isoflurane diffused into a $30 \%$ oxygen and $70 \%$ nitrogen mixture. After confirming anesthetization via tail pinch, mice received an intraperitoneal injection of $25 \mathrm{U}$ heparin in $250 \mu 1$ saline intravenous solution. Mice were then transcardially perfused at 160 $\mathrm{mmHg}$ with $0.01 \mathrm{M}$ phosphate buffered saline (PBS) containing $25 \mathrm{U} / \mathrm{mL}$ of heparin followed by $4 \%$ paraformaldehyde (PFA) in 0.01M PBS at physiological $\mathrm{pH}$. The polyurethane (PU4ii, VasQtec) cast solution was prepared by adding $5 \mathrm{~g}(\boldsymbol{\ell})$ of polyurethane resin with $3 \mathrm{~g}(\boldsymbol{\ell})$ of

methyl ethyl ketone containing approximately $10 \mathrm{mg}$ of blue pigment. Shortly before perfusion of the cast resin, $0.8 \mathrm{~g}(\boldsymbol{\ell})$ of polyurethane hardener was added to the solution then immediately perfused into mice at $160 \mathrm{mmHg}$. Resin perfused mice were kept at room temperature for 4 hours to facilitate the hardening of the polyurethane resin. 


\section{Vascular Corrosion Cast Processing}

Perfused mice were decapitated and the skin was removed from the skull with dissecting scissors. The isolated skull was decalcified by immersion in $20 \mathrm{~mL}$ of $8 \%$ formic acid diluted in Milli-Q water for $5 \mathrm{~h}$ at $37{ }^{\circ} \mathrm{C}$. After decalcification, the skull was rinsed with distilled water then immersed in $20 \mathrm{~mL}$ of potassium hydroxide solution diluted in Milli-Q water for $4 \mathrm{~h}$ at $37^{\circ} \mathrm{C}$. The skull was rinsed with distilled water and the brain carefully extracted with a pair of iris scissors and forceps. The extracted brain was macerated by immersion into $20 \mathrm{~mL}$ of $8 \%$ potassium hydroxide diluted in Milli-Q water at $37{ }^{\circ} \mathrm{C}$ overnight. Macerated tissue was removed from the cerebrovascular cast by several washes with Milli-Q water, until only the casted vessels remained. At this point, the clean cerebrovascular cast was lyophilized with a benchtop freeze dryer system (Labconco) operating at $-54{ }^{\circ} \mathrm{C}$ and a vacuum pressure of $0.0025 \mathrm{mBar}$. To enhance the radio opacity of the cerebrovascular casts, $6 \mathrm{~mL}$ solution of $2 \%$ osmium tetroxide diluted in Milli-Q water was embedded onto the polyurethane cast by immersion overnight at $4{ }^{\circ} \mathrm{C}$. Osmium embedded casts were mounted with cyanoacrylate adhesive on custom-made hexagonal pedestals cut from Plexiglas.

\section{Micro-computerized Tomographic Imaging}

Tomographic images of the vascular network of the whole brain were acquired using a SkyScan $1272 \mu \mathrm{CT}$ (Bruker) operating at $35 \mathrm{kV}$ and $200 \mu \mathrm{A}$ with no filter and a frame size of 4032-by-2686. Acquisition parameters were as follows, a pixel size of $2 \mu \mathrm{m}$, and exposure settings that produce a maximum transmission of $35-40 \%$, minimum of $80-90 \%$, and an average of $65-75 \%$ through the sample. Tomographic images of each vascular cast were acquired over the total $360^{\circ}$ of the sample at a step size of $0.05^{\circ}$, and averaged by 5 images per 
rotation step. Before further processing, each dataset was corrected for misalignments, beam hardening, and ring artifacts then converted into a coronal image series. Each acquisition routine produced 7200 projection images and 4032 images in coronal series.

\section{Anatomical Selection of Volume of Interest}

Datasets containing the whole brain vasculature in coronal series were accessed on CTan (Bruker) to isolate the volume of interest (VOI). Guided by the Allen Mouse Brain Atlas (Lein et al. 2007), VOIs over the medial orbital prefrontal cortex (MO PFC), somatosensory cortex (SS CTX), cingulate cortex (CC), entorhinal cortex (ENT CTX), dentate gyrus (DG) and hippocampal CA1 and CA3 were located and selected by tracing. The traced regions were made in square selections of approximately $1.5 \mathrm{~mm}$ and dynamically interpolated in the $\mathrm{Z}$ direction for $1.5 \mathrm{~mm}$. These selected VOIs were converted into a BMP formatted series and saved as pre-processed VOIs.

\section{Image Processing and Optimization for Volume of Interest}

For a detailed description of VOI dataset optimization please refer to our previous report (Quintana et al. 2019). Briefly, VOI datasets were optimized for further image analysis using Image (NIH). Each VOI was cropped in three-dimensions to 500 x 500 x 500 (MO PFC, SS CTX, ENT CTX, CA1, and CA3) or $400 \times 400 \times 400$ (CC and DG) then rotated so that a preselected landmark was co-registered by rigid transformation across all datasets. For each dataset, the image series was duplicated and saved as TIFF formatted images. With the 3D/2D skeletonization plugin on ImageJ, the first duplicated dataset was used to produce centerlines 
of the vascular structures. The second duplicated dataset was filtered by maximum pixel intensity of 160 to produce a dataset with pixel intensities 160 and below. Finally, both image series containing the vessel centerlines and capped pixel intensity were merged so that the final dataset contains vessel structures composed of pixel intensities 160 and below and centerlines of vessels at an intensity of 255 .

\section{Vascular Network Analysis}

Datasets were analyzed with Imaris (Bitplane) software. Vascular networks were identified and quantified with the Imaris Filament Trace function using the automatic filament and path detection algorithm. Filament trace approximates the diameter of blood vessels in a volumetric data set by calculating the diameter of the largest sphere that fits within the cross sectional area of a vessel segment. We used the Loops algorithm and automatic threshold during the tracing procedure for an accurate identification of circular pathways between vessel segments. Traced networks were automatically re-centered to the centerlines of the vascular structures that were inserted into the volumetric dataset in each VOI. The identified vascular networks were manually inspected for errors that occurred during the tracing process before measurements were generated from the mapped vessel network(s). These measurements included vessel diameter, length, volume, area, tortuosity, branch level, and angle in degrees.

\section{Experimental Design and Statistical Analysis}

Power analysis for ANOVA designs indicated a sample size of 4 mice per group (power $=0.999)$ for an effect size of $\Delta=1.25$. To study the effects of aging and disease on the 
cerebrovasculature over the whole-brain, analyses were performed on mice at 3-months (WT, $\mathrm{n}=4 ; 3 \mathrm{xTg} \mathrm{AD}, \mathrm{n}=4$ ), 6-months (WT, $\mathrm{n}=3$; 3xTg AD, $\mathrm{n}=4$ ), 12-months (WT, $\mathrm{n}=4$; 3xTg $\mathrm{AD}, \mathrm{n}=3$ ), and 24-months (WT, $\mathrm{n}=4 ; 3 \times \mathrm{Tg} \mathrm{AD}, \mathrm{n}=4)$ of age. Analyses of the cerebrovasculature in specific brain regions were performed on mice at 3 -months (WT, $n=4$; 3xTg AD, n = 5), 6-months (WT, n = 7; 3xTg AD, n = 5), 12-months (WT, $\mathrm{n}=5$; 3xTg AD, $\mathrm{n}$ $=4$ ), and 24-months (WT, $\mathrm{n}=5 ; 3 \times \mathrm{Tg} \mathrm{AD}, \mathrm{n}=4$ ). All values are presented as mean $\pm \mathrm{SEM}$ for all data provided. All statistical analyses were performed on GraphPad Prism 8.4.2. The planned comparison for all data were the effects of genotype at each age. Before performing planned comparisons, 2-way ANOVA analyses of Genotype by Age were used to qualify the data from each of the experimental measurements. Details of each 2-way ANOVA, including degrees of freedom, F-values, and p-values are reported in the Results section of the text. To compare means, significant 2-way ANOVAs were probed for effects of Genotype with planned student's t-test at each age. All student's t-tests are two-tailed unless specified. Analysis of trend was performed with linear regression analysis. Details of each linear regression analysis, including $\mathrm{R}^{2}$-values and $\mathrm{p}$-values are reported in the Results section of the text. Values of $\mathrm{p}<$ 0.05 were considered as significant.

\section{Results}

We have reported previously a procedure for the acquisition and analysis of the entire brain cerebrovascular system at a microvessel-relevant resolution, to provide a detailed characterization of the morphological and topological properties of vessels and their networks (Quintana et al. 2019). In the present study, we use vascular corrosion casts to acquire data 
from complete networks of cerebral blood vessels in a state that preserves native connectivity. We performed an in-depth analysis of the cerebrovasculature in WT $(n=20)$ and 3xTg AD (n = 20) mice by assessing the function, morphology, topology, and complexity of vascular networks over the entire brain then focused on prominent brain regions critically affected during the progression of $\mathrm{AD}$ (Figure 6.1). Indeed, geometric characterization of the vasculature has provided valuable insight for identifying fundamental, yet broad changes to the cerebrovasculature during age and AD. However, these broad changes may only be detectable when the pathology of the cerebrovascular system is severe. It is likely that early changes to the organization of the vascular network cause dysregulated functional connectivity before the onset of detectable changes to broad parametric properties such as volume density of cumulative length. It is firmly accepted that with the progression of $\mathrm{AD}$, the development of vascular dysfunction is at increased risk (Santos et al. 2017). It is unknown whether the $3 \times \mathrm{Tg} \mathrm{AD}$ mouse line manifests functional deficits and degeneration of the vasculature that is similar to human patients with the disease. Therefore, our first goal was to determine if the 3xTg AD mouse line developed functional deficits in cerebral blood flow during aging and disease progression.

The mice used in this study were $3.0 \pm 0.5,6.0 \pm 0.3,12 \pm 0.6$, and $24 \pm 0.6$ months of age and had an average body temperature of $35.9 \pm 0.8{ }^{\circ} \mathrm{C}$. Systolic $(F(3,49)=5.969, p=$ 0.0015, 2-way ANOVA $)$ and diastolic $(F(3,49)=36.71, p<0.0001,2$-way ANOVA $)$ blood pressure fell within physiological range (Whitesall et al. 2004; Wilde et al. 2017). There were no group differences in blood pressure between WT and 3xTg AD mice at either 3- nor 6months of age. However, at later ages, both systolic (12-months, $t(12)=2.41, p=0.01$, $t$-test; 24-months, $t(16)=5.99, p<0.0001, t$-test $)$ and diastolic (12-months, $t(12)=3.31, p=0.001$, 


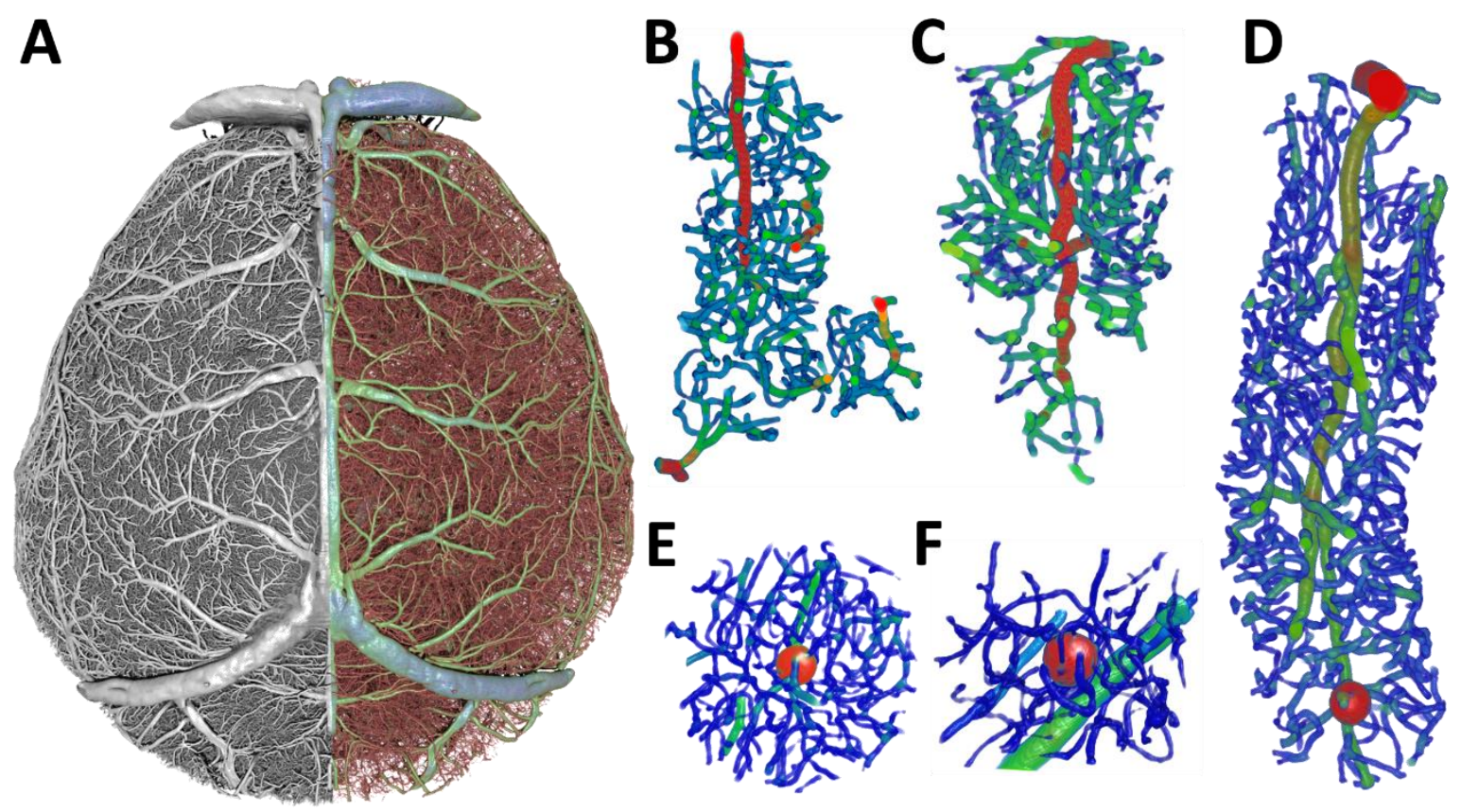

Figure 6.1. The cerebrovasculature in 3xTg AD mice develops pathology. Reconstructed cerebrovascular corrosion cast in three-dimensions, color-coded by vessel diameter of (A) whole brain, (B-E) cortex, and (F) hippocampus. Color-coded penetrating arteriole demonstrating (B) normal morphology compared to a (C and D) tortuous vessel from a 3xTg AD mouse. Color-coded vasculature demonstrating (D) arteriolar aneurysm and $(\mathrm{E}$ and $\mathrm{F})$ capillary aneurysm from $3 \mathrm{xTg} \mathrm{AD}$ mice. 
$t$-test; 24-months, $t(16)=13.58, p<0.0001$, $t$-test ) blood pressure were lower in $3 \times \mathrm{Xg} \mathrm{AD}$ mice compared to age-matched WT mice (Figure 6.2A and B). The mean arterial pressure (MAP) is the perfusion pressure that facilitates the delivery of blood to the brain. Inadequate MAP can result in ischemia to the brain and other organs (Wehrwein and Joyner 2013). Mean arterial pressure is determined by cardiac output during systole and the net driving force of blood back to the heart during diastole. $\operatorname{MAP}(F(3,49)=5.711, p=0.002,2$-way ANOVA) was lower in 3xTg AD mice at 12-months $(t(12)=2.0, p=0.05$, $t$-test $)$ and 24-months $(t(16)=$ 5.77, $p<0.0001, t$-test) of age compared to age-matched WT mice (Figure 6.2C). Although, we observed only a small reduction in heart rate $(F(3,49)=1.935, p=0.13,2$-way ANOVA $)$ at 3-months in 3xTg $\mathrm{AD}(t(14)=2.27, p=0.02$, t-test $)$ compared to WT mice, we found no change at 6-, 12- and 24-months of age (Figure 6.2D).

\section{Cerebrovascular hypoperfusion occurs early and progresses with age in 3xTg AD mice}

It is well documented that deficits in $\mathrm{CBF}$ occur in patients with $\mathrm{AD}$ (Thomas et al. 2015; Love and Miners 2016; Dong et al. 2018; Shi et al. 2019; Daulatzai 2017; Göttler et al. 2019; Zhai et al. 2016; Marrif and Juurlink 1999; Verclytte et al. 2016). However, it is yet unknown whether these deficits occur in 3xTg AD mice and if so, when these deficits take place during the disease. We measured global $\operatorname{CBF}(F(3,45)=4.095, p=0.01,2$-way ANOVA $)$ in 3-, 6-, 12-, and 24-month male WT and 3xTg AD mice using laser speckle flowmetry (Figure 6.2E). We found that deficits in CBF began early and progressed with age in 3xTg AD mice. At 3-months of age, 3xTg AD mice demonstrated similar $\mathrm{CBF}(t(12)=1.45, p=0.15$, $t$-test $)$ to age-matched WT mice. However, at 6-months of age, 3xTg AD mice demonstrated a deficit of $\mathrm{CBF}(t(11)=3.54, p=0.0009, t$-test $)$ relative to age-matched WT mice (Figure 6.2F). We 
found that the CBF deficit in 3xTg AD mice persisted at 12-months $(t(15)=3.70, p=0.002$, $t$-test $)$ and also at 24-months $(t(12)=5.99, p<0.0001, t$-test $)$ of age (Figure 6.2F).

\section{Whole-brain analysis revealed early structural changes to the angioarchitecture occur before the onset of vascular degeneration in 3xTg AD mice}

Our first goal was to characterize the extent of vascular disruption over the whole brain with age and the progression of $\mathrm{AD}$. To evaluate the evolution of the structural changes to the angioarchitecture, we calculated the overall means of the geometric properties over all vessels of the brain. Morphometric analysis of the vasculature over the entire brain indicated that the average vessel diameter at 3-months $(t(7)=1.897, p=0.05$, $t$-test $)$ and 6-months $(t(6)=2.310$, $p=0.03, t$-test $)$, but not at $12-(t(6)=0.135, p=0.4, t$-test $)$ and $24-(t(7)=1.234, p=0.13, t$ test) months were statistically different between the two genotypes (Figure 6.3A). A change to the average vessel diameter may indicate a compensatory adaptation of the vascular network, likely caused by either chronic injury to vessels or via disrupted angiogenesis.

Arborization of the vasculature is achieved by the growth of new vessel segments. With the growth of new segments, vascular density increases, enhancing the exchange of waste and nutrients with the surrounding tissue. Therefore, we quantified the total number of vessel segments $(F(3,22)=17.53, p<0.0001,2$-way ANOVA $)$ of the whole brain and found early changes to the number of segments in 3xTg AD mice that began at 6-months of age. At 6months of age, the cerebrovascular network in 3xTg AD mice was composed of a greater number of vessel segments $(t(6)=2.476, p=0.02$, $t$-test ) than in age-matched WT mice (Figure 6.3B). The total number of vessel segments in $3 \mathrm{xTg} \mathrm{AD}$ mice remained increased $(t(6)=2.222$, $p=0.04, t$-test ) at 12-months of age. Interestingly, at 24-months of age, the total number of 


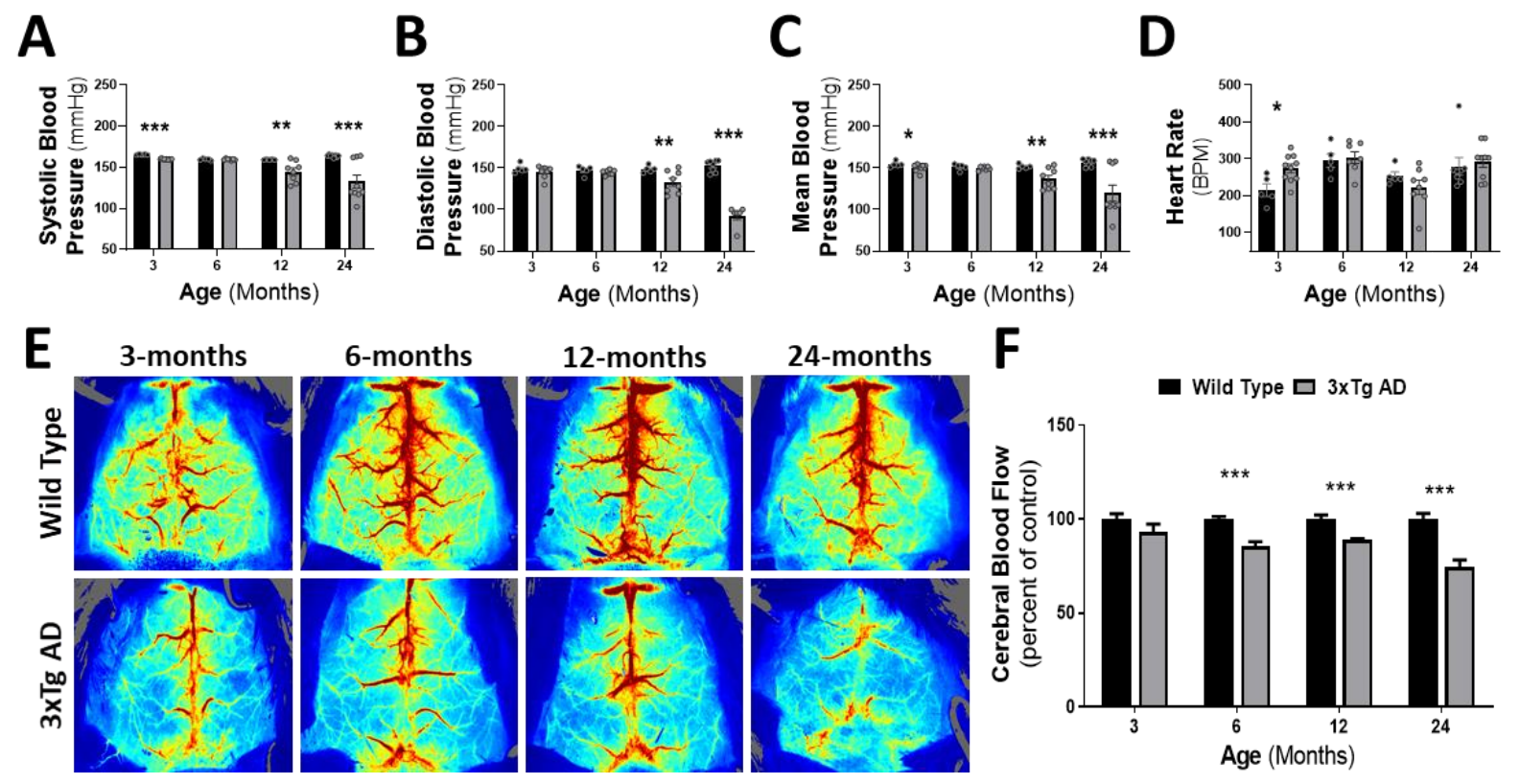

Figure 6.2. Progressive decline in cerebral blood flow in 3xTg AD mice. Bar graph(s) (mean \pm SEM) with individual data points depicting (A) systolic, (B) diastolic, (C) mean arterial blood pressure, and (D) heart rate in 3- (WT, $\mathrm{n}=5$; 3xTg AD, $\mathrm{n}=10), 6-(\mathrm{WT}, \mathrm{n}=$ 5; 3xTg AD, $\mathrm{n}=7$ ), 12- (WT, $\mathrm{n}=5 ; 3 \times \mathrm{Tg}$ AD, $\mathrm{n}=8)$, and 24- (WT, $\mathrm{n}=8$; 3xTg AD, $\mathrm{n}=$ 5) month mice. Cerebral blood flow (E) color coded by mean flux value in WT and 3xTg AD mice. Bar graph (mean \pm SEM) depicting cerebral blood flow as percentage of agematched control from 3- (WT, $\mathrm{n}=5$; 3xTg AD, $\mathrm{n}=8$ ), 6- (WT, $\mathrm{n}=5 ; 3 \mathrm{xTg}$ AD, $\mathrm{n}=6$ ), 12 (WT, $\mathrm{n}=5 ; 3 \times \mathrm{Tg}$ AD, $\mathrm{n}=9$ ), and 24- (WT, $\mathrm{n}=5 ; 3 \times \mathrm{Tg}$ AD, $\mathrm{n}=8)$ month mice. To compare means, significant 2-way ANOVAs were probed for effect of Genotype with planned student's t-test at each age $(*, \mathrm{p}<0.05 ; * *, \mathrm{p}<0.01 ; * * *, \mathrm{p}<0.001)$. 
vessel segments in 3xTg AD mice was substantially lower $(t(7)=6.806, p=0.0005$, $t$-test $)$ than the number of segments recorded in age-matched WT mice (Figure 6.3B).

These data support the notion that early changes to the cerebrovasculature are driven by compensatory adaptations to the network composition in response to chronic vascular injury associated with the disease. An essential physical property of blood vessels is the surface area they possess. The total surface area a vascular network possesses is directly proportional to the efficacy of solute exchange between the parenchyma and peripheral circulation. Regression analysis of the total surface area $(F(3,22)=4.882, p=0.009,2$-way ANOVA $)$ of all vessels revealed a linear trend that decreased with age in $3 \times \operatorname{Tg} \mathrm{AD}$ mice $\left(R^{2}=0.55, p=0.0013\right)$, whereas the linear trend in WT mice increases with age $\left(R^{2}=0.29, p=0.036\right)$. The total surface area of vessels was most affected at 24-months in 3xTg AD mice. At this age, the complete cerebrovascular network provided a total surface area that was substantially less $(t(7)=3.867$, $p=0.0008, t$-test) in 3xTg AD mice than in age-matched WT mice (Figure 6.3C).

Given these findings, since the majority of the surface area is provided by the microvasculature (Gould et al. 2017), we interpret these data to suggest that the age-dependent changes to the cerebrovasculature of $3 \mathrm{xTg} \mathrm{AD}$ mice are largely driven by microvascular degeneration. In order to determine the extent of vascular degeneration in 3xTg AD mice, we measured the total vascular volume of the entire brain with age. We found no early differences in the total volume occupied by vessels between the genotypes (Figure 6.3D). However, a regression analysis of these changes revealed an age-dependent reduction of volume in $3 \mathrm{xTg}$ AD mice (Figure 6.3D). In contrast, WT mice demonstrated a pattern of vessel volume that increased with age. When we compared means between genotypes, the total volume of vessels $(F(3,22)=4.621, p=0.01,2$-way ANOVA $)$ was concordant at $3-(t(7)=0.84, p=0.35, t$-test $)$, 
6- $(t(6)=0.59, p=0.14, t$-test $)$, and 12- $(t(6)=0.29, p=0.15, t$-test $)$ months of age in $3 \times \mathrm{Tg}$ AD and age-matched WT mice. At 24-months of age, 3xTg AD mice demonstrated a marked reduction of the total volume occupied by vessels $(t(7)=3.84, p=0.0009, t$-test $)$ compared to age-matched WT mice (Figure 6.3D). These data support the notion that early structural changes to the organization of the cerebrovasculature occur before the onset of large-scale changes, which may result in vascular degeneration.

We measured the average intervessel distance in three-dimensions as a proxy to assess the adequacy of vascularization. We found intervessel distance $(F(3,22)=28.34, p=0.0001$, 2-way ANOVA) to be a profoundly compromised property of the angioarchitecture in all ages of 3xTg AD mice (Figure 6.3E). At 3-months of age, the average distance between vessels was $\operatorname{larger}(t(7)=6.40, p<0.0001, t$-test $)$ in $3 \times \mathrm{Tg} \mathrm{AD}$ mice than in WT mice. Increased intervessel distance in 3xTg AD mice was also evident at 6-months $(t(6)=7.13, p<0.0001, t$-test $)$ and to 12-months $(t(6)=4.01, p=0.0006, t$-test $)$ of age compared to age-matched WT mice. Interestingly, we found that by 24 -months, intervessel distance $(t(7)=4.86, p<0.0001$, $t$-test $)$ was reduced in 3xTg AD mice than WT mice (Figure 6.3E). Regression analysis revealed that intervessel distance is inversely associated with age in $3 \mathrm{xTg}$ AD mice $\left(R^{2}=0.64, p=0.003\right)$ while positively associated with age in WT mice $\left(R^{2}=0.82, p<0.0001\right)$. These data indicate an early deficit of vessel density that persists to later stages of progression in 3xTg AD mice.

\section{Topological analysis of the vascular network over the whole brain revealed disrupted network organization in 3xTg AD mice that progresses with age}

Since morphometric analyses of the angioarchitecture provided evidence of early structural changes to the vasculature that preceded loss of vessels, we assessed the topology of 
the vascular network over the entire brain. We used a topological analysis to evaluate the organization and complexity of the vascular network with age. We first examined the connectedness of the vascular network. Consistent with the literature (Gross 2006; Sheth and Liebeskind 2014), we observed a dense network of vessels with elevated pathway redundancy in both WT and 3xTg AD mice at all ages.

We used an algorithm to calculate the Euler number and pathway redundancy of the vascular network in three-dimensions. The Euler number is calculated based on the classification of several morphological events: islands, bridges, and holes. We first quantified the number of connections in the vascular network by counting vessel-to-vessel junctions $(F(3,22)=10.96, p=0.0002,2$-way ANOVA $)$ within the entire brain. At 6-months of age, the number of vessel junctions in 3xTg AD mice were greater $(t(6)=2.72, p=0.05, t$-test $)$ than the number of junctions in WT mice. While we observed no difference in the number of connections at 12-months $(t(6)=0.74, p=0.46, t$-test $)$ between genotypes, by 24 -months of age, the total number of connections in $3 \mathrm{xTg}$ AD mice were lower $(t(7)=5.61, p<0.0001, t$ test) than those in WT mice (Figure 6.3F).

The cerebrovascular network provides a profound number of redundant pathways for the delivery of blood to any given region. These redundant pathways maintain perfusion by providing an alternate route to communicate shunted blood to a particular region. These collateral pathways for blood flow lend to the resilience of the network to maintain adequate blood delivery during an event of a pathway communicating blood becomes compromised. We next evaluated the redundancy of pathways $(F(3,22)=3.694, p=0.02,2$-way ANOVA $)$ within the vascular network by calculating the connectivity value and compared this across genotype. The connectivity value is topologically defined as the maximum number of cuts that can be 
performed on a network before splitting into two isolated subnetworks (Nyengaard 1999). Regression analysis of pathway redundancy indicated an inverse correlation $\left(R^{2}=0.32, p=\right.$ 0.02 ) with age in $3 \times \mathrm{Tg}$ AD mice. In contrast, pathway redundancy in WT mice correlated positively $\left(R^{2}=0.17, p=0.15\right)$ with age. Connectivity analysis indicated an elevated $(t(7)=$ 2.37, $p=0.03$, t-test) number of redundant pathways in 3xTg AD mice at 3-months of age. These pathways remained elevated $(t(6)=2.25, p=0.05$, $t$-test $)$ in $3 \times \mathrm{Tg} \mathrm{AD}$ mice relative to WT mice at 6-months of age. Interestingly, these differences were absent at 12 -months $(t(6)=$ 1.37, $p=0.18, t$-test $)$ and 24-months $(t(7)=1.27, p=0.21, t$-test $)$ of age (Figure 6.3G).

We next evaluated the complexity of the vascular network with age and progression of AD. We used Kolmogorov box counting method for fractal dimensions (Quintana et al. 2019) and found that the complexity of the vascular network progressively declines with the progression of age in 3xTg AD mice whereas in WT mice partially increases with age. The fractal dimension of a vascular structure quantitatively evaluates its branching patterns and their space-filling ability by assessing the relationship between its branches of like hierarchical order and the number of levels the network branches. Analysis of fractal dimension value $(F(3,22)=10.53, p=0.0002,2$-way ANOVA $)$ indicated no difference between the two genotypes at 3-months of age. However, at 6-months of age, the complexity of the vascular network of 3xTg AD mice was greater $(t(6)=2.96, p=0.007$, $t$-test $)$ than of age-matched WT mice. Following 6-months, the vascular network complexity of 3xTg AD mice begins to trend downward with age $\left(R^{2}=0.79, p=0.0002\right)$ whereas in WT mice, network complexity trends upward with age $\left(R^{2}=0.39, p=0.05\right)($ Figure $6.3 \mathrm{H})$. By 24-months of age, network complexity in $3 \mathrm{xTg} \mathrm{AD}$ mice was severely compromised $(t(7)=4.42, p=0.0002$, $t$-test $)$ when compared to age-matched WT mice (Figure 6.3H). 


\section{Age and disease related changes to vessel hierarchy over the whole brain occur in $3 \times \mathrm{Tg}$}

AD and WT mice

Specific differences at the level of vessel hierarchy may exist between age and genotype. We generated thickness maps of all vessels of the brain to classify and analyze vascular volume as a function of vessel diameter. Such that, the sum volume is calculated for all vessels of each successive diameter. We found that the distribution of vessel volume in 3xTg AD mice demonstrated large changes that occurred with age and the progression of the disease (Figure 6.3I - L). The distribution of vessels as a function of diameter depicted that the largest changes between genotypes per age was accounted for by vessels with an average diameter between 2 - 40 $\mu m$ (Figure 6.3I-L). When we compared the mean volume per vessel diameter between the two genotypes at each age we found that vessels with an average diameter between $2-20 \mu m$ appeared to decrease with age in 3xTg AD mice whereas in WT mice, these vessels increased with age (Figure 6.3M-P). Interestingly, 3xTg AD mice demonstrated an early increase $(F(7,40)=6.0, p<0.0001,2$-way ANOVA) of vessels with an average diameter between $2-20 \mu \mathrm{m}$ at 3 -months of age. This observation was also seen at 6months of age $(F(7,40)=10.97, p<0.0001,2$-way ANOVA $)$, relative to age matched WT mice (Figure 6.3M and N). However, by 12-months of age, vessels with an average diameter between $2-20 \mu \mathrm{m}$ was not significantly different $(F(7,40)=1.99, p=0.08,2$-way ANOVA) between the two genotypes and remained indifferent $(F(7,40)=1.49, p=0.19,2$-way ANOVA $)$ by 24-months of age (Figure $6.3 \mathrm{O}$ and $\mathrm{P}$ ). Furthermore, larger vessels with an average diameter between $20-40 \mu \mathrm{m}$ were reduced at later ages in 3xTg AD mice. Although we found no difference in the population of larger vessels at 3- and 6-months of age, we observed reduced 

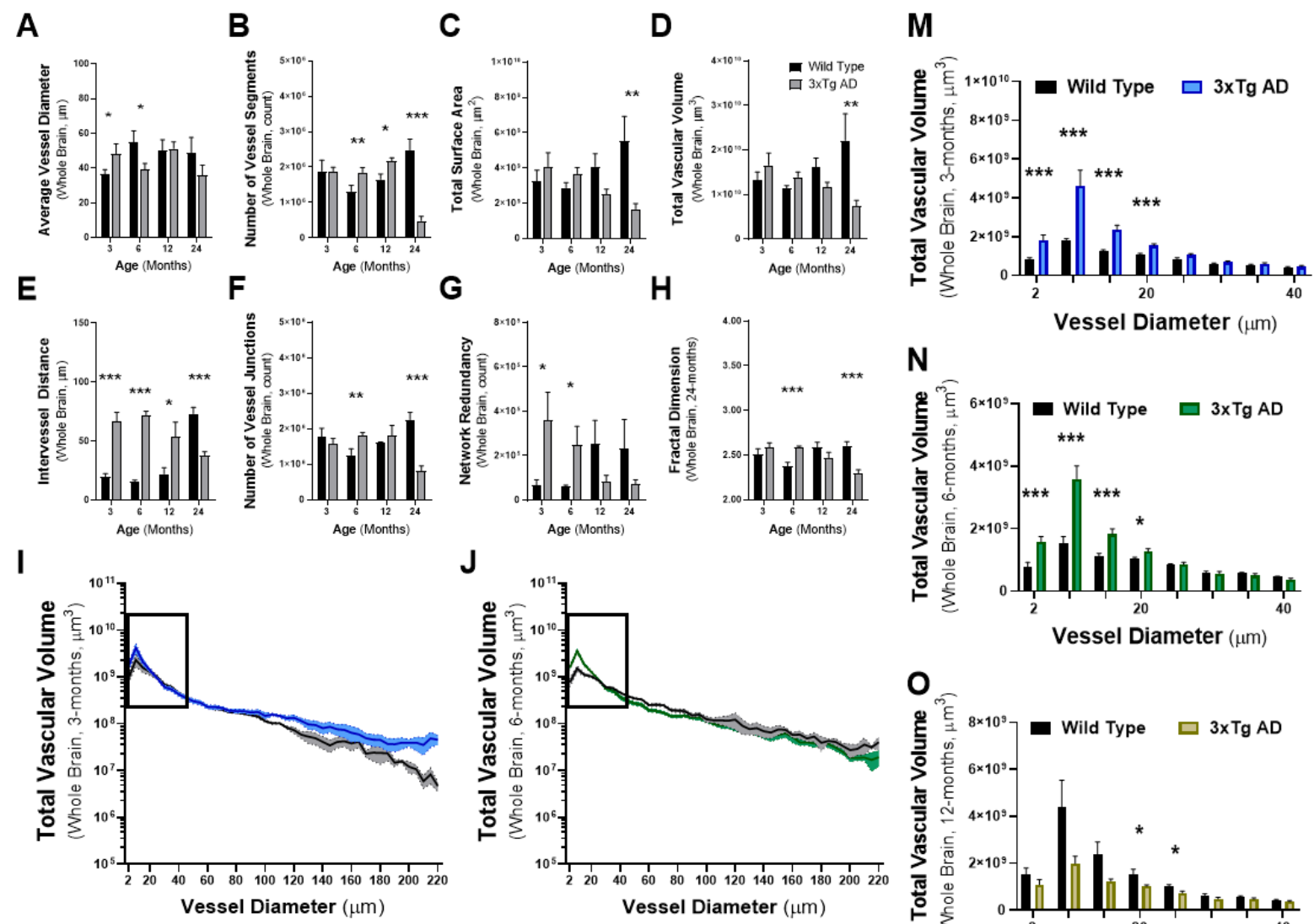

K
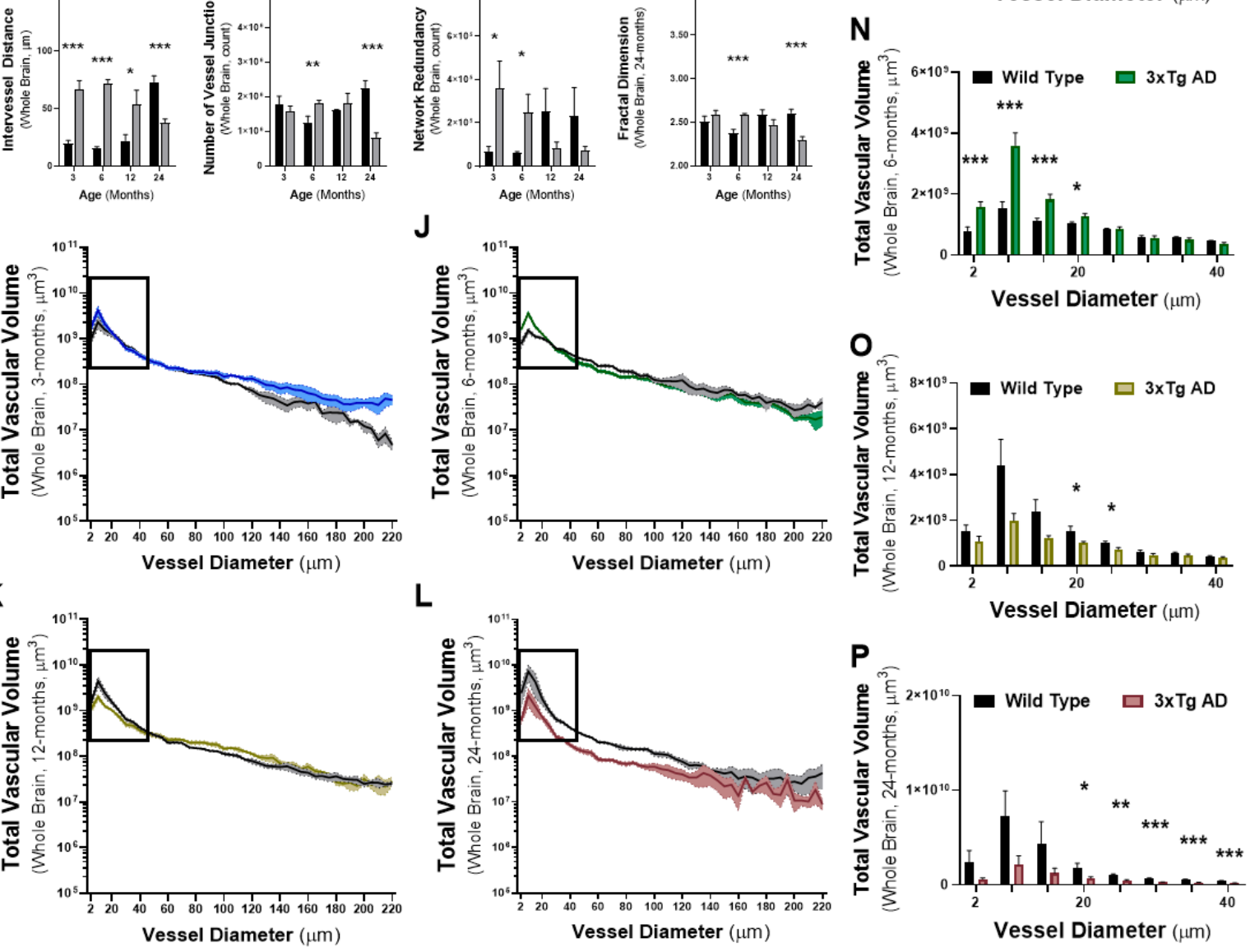
Figure 6.3. Brain-wide vascular degeneration in $3 \times \mathrm{Tg}$ AD mice that progresses with age. Bar graph(s) (mean \pm SEM) depicting (A) average vessel distance, (B) number of vessel segments, (C) total surface area, (D) total vascular volume, (E) intervessel distance, (F) number of junctions, $(\mathrm{G})$ network redundancy, and $(\mathrm{H})$ fractal dimension from 3- (WT, $\mathrm{n}=4 ; 3 \times \operatorname{Tg} \mathrm{AD}, \mathrm{n}=4), 6-(\mathrm{WT}, \mathrm{n}=3 ; 3 \times \operatorname{Tg} \mathrm{AD}, \mathrm{n}=4)$, 12- (WT, $\mathrm{n}=4 ; 3 \times \mathrm{Tg} \mathrm{AD}, \mathrm{n}=3$ ), and 24- (WT, $n=4 ; 3 x T g A D, n=4)$ month old mice. Line graph(s) (mean \pm SEM) depicting total vascular volume as a function of average vessel diameter from (I) 3- (WT, $n$ = 3; 3xTg AD, n = 4), (J) 6- (WT, n = 3; 3xTg AD, n = 4), (K) 12- (WT, n = 4; 3xTg AD, $\mathrm{n}=3$ ), and (L) 24- (WT, $\mathrm{n}=4$; 3xTg AD, $\mathrm{n}=4$ ) month old mice. Bar graph(s) (mean \pm SEM) depicting expanded data denoted by the box inserts (M-P). To compare means, significant 2-way ANOVAs were probed for effects of Genotype with planned student's ttest at each age or vessel diameter $\left(*, p<0.05 ; *^{*}, \mathrm{p}<0.01 ; * * *, \mathrm{p}<0.001\right)$. 
vessels with an average diameter with $20-40 \mu \mathrm{m}$ by 24 -months of age in $3 \times \mathrm{Tg}$ AD mice relative to WT mice.

These data indicate that the cerebrovascular network in 3xTg AD mice undergo an early vascularization by 3 -months of age then begin to progressively decline by 12 -months of age (Figure 6.4). To better demonstrate these changes with age, we measured the total volume of capillaries and non-capillaries for both genotypes at each age. Regression analysis revealed an age-associated decline $\left(R^{2}=0.53, p=0.002\right)$ of the total capillary volume in the brain of $3 \times \mathrm{Tg}$ AD mice, whereas in WT mice, the total capillary volume increased with age $\left(R^{2}=0.47\right.$, $p=0.006)$. When we compared means across genotype per age group $(F(3,21)=6.41, p=$ 0.003, 2-way ANOVA), we found that by 3-months the total volume of capillaries was increased $(t(6)=3.84, p=0.01, t$-test $)$ in $3 \times \mathrm{Tg} \mathrm{AD}$ mice compared to age-matched WT mice (Figure 6.4A). The total capillary volume remained elevated $(t(6)=3.70, p=0.01, t$-test $)$ by 6 -months in 3xTg AD mice, relative to age-matched WT mice (Figure 6.4A). By 12-months of age, the total volume of capillaries in 3xTg AD mice did not differ $(t(6)=1.70, p=0.14$, $t$-test $)$ from that in age-matched WT mice. At 24-months of age the total volume of capillaries was lower $(t(7)=2.37, p=0.05, t$-test $)$ in $3 \times \mathrm{Tg} \mathrm{AD}$ mice compared to age-matched WT mice (Figure $6.4 \mathrm{~A})$.

Similarly, regression analysis of the total volume of non-capillaries revealed an ageassociated decline $\left(R^{2}=0.76, p<0.0001\right)$ in $3 \times \mathrm{Tg}$ AD mice, whereas in WT mice, the total volume of non-capillaries increased with age $\left(R^{2}=0.15, p=0.15\right)$. When we compared means across genotype at each age group $(F(3,21)=6.29, p=0.003,2$-way ANOVA), we found no significant difference at 3- $(t(6)=1.79, p=0.06, t$-test $)$ and 6-months $(t(6)=0.17, p=0.43, t$ test) months of age (Figure 6.4B). However, at 12-months of age, the total volume of non- 
capillaries was reduced $(t(6)=1.98, p=0.05$, $t$-test $)$ compared to WT mice and continued to decline $(t(7)=3.14, p=0.009, t$-test $)$ by 24-months of age (Figure 6.4B).

\section{Cerebrovasculature of the hippocampal formation during ageing and the progression of} AD

We next evaluated the angioarchitecture of the hippocampal formation by measuring the composition and topology of the vascular network in order to characterize regional pathology to the vasculature network of the hippocampus and to establish a time-course for the onset and progression of vascular disruption in $3 \times \mathrm{Tg} \mathrm{AD}$ mice.

The hippocampus develops age-dependent neuropathy in 3xTg AD mice (Billings et al. 2005; Oddo et al. 2003b; Oddo et al. 2003a; Giménez-Llort et al. 2007; España et al. 2010) and is the first region of the brain to develop A $\beta$-plaque (Oddo et al. 2003b; Oddo et al. 2003a). Furthermore, the hippocampus is a major region of the brain for the deposition of A $\beta$ in hAPPJ20, APP/PS1, and 3xTg AD mice (Oddo et al. 2003a; Oddo et al. 2003b; Whitesall et al. 2004). To characterize the region-specific and age-associated, disease-related pathology to the vascular network of the hippocampus, we analyzed the angioarchitecture in subfield(s) CA1, CA3 and DG of the hippocampus proper and the ENT CTX of the hippocampal formation in 3xTg AD and WT mice.

Our initial assessment consisted of morphometric analyses of all vessels to evaluate the breadth of vessel branching (vessel segment count and number of vessel-to-vessel junctions), regional vascularization (total vessel volume and vessel surface area), and network efficacy (intervessel distance). We found that changes to the angioarchitecture in the hippocampus 

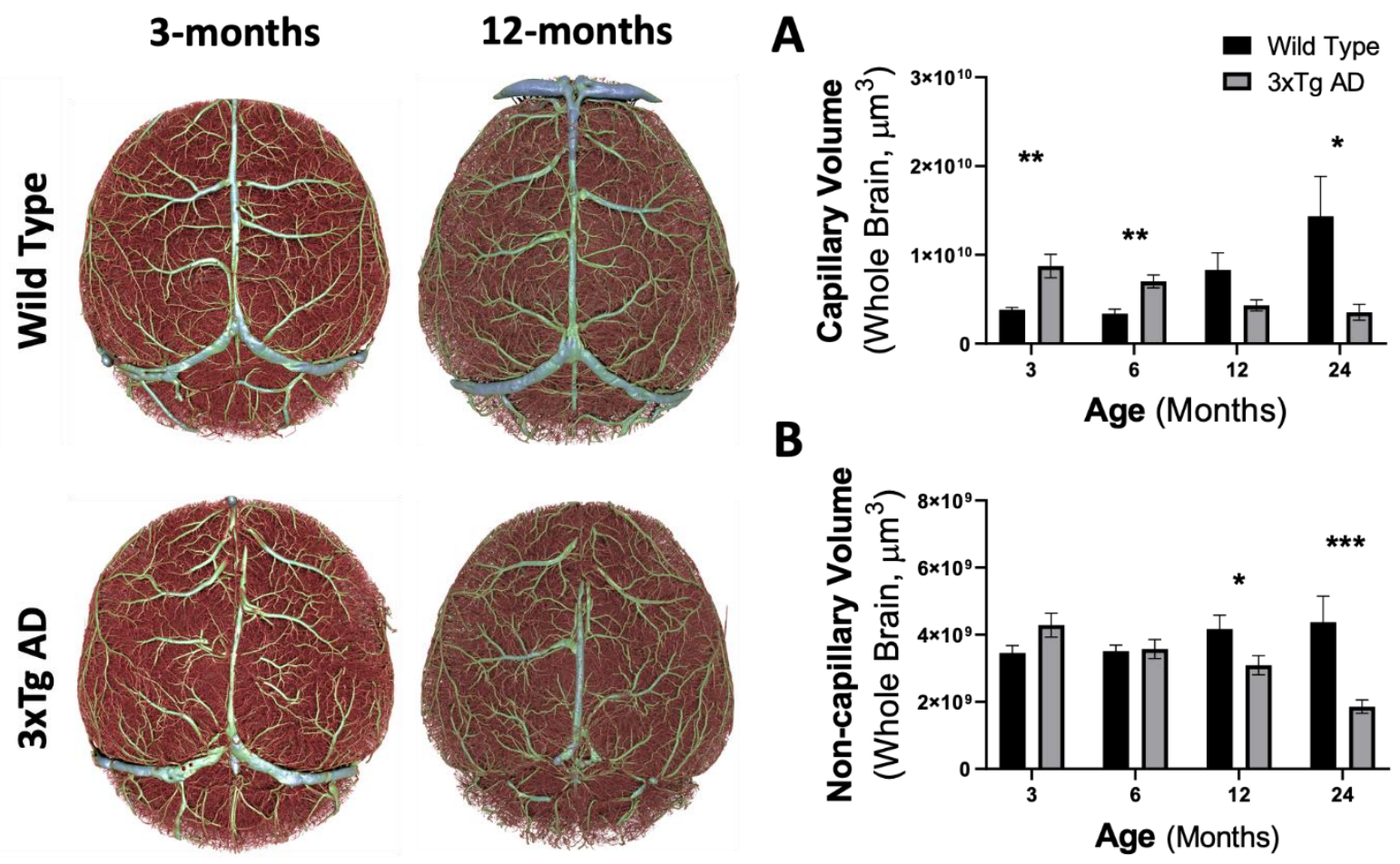

B

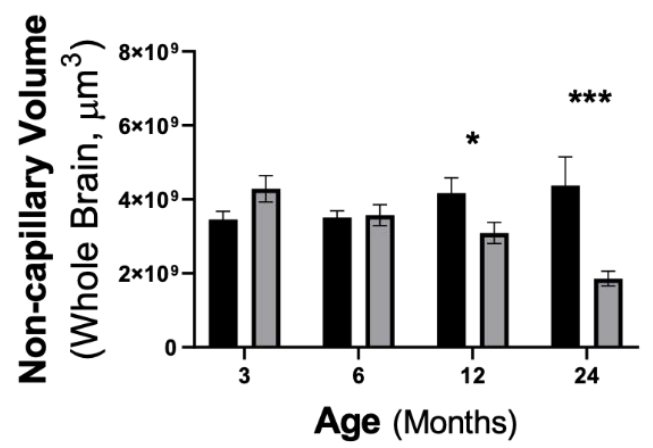

Figure 6.4. Topological analysis of the cerebrovasculature of the entire brain describes changes that progress with age in 3xTg AD mice. Reconstructed cerebrovascular corrosion cast, color-coded by vessel diameter from a 3-month and 12-month WT and 3xTg AD mouse. (A) The total volume of capillaries and (B) non-capillaries in WT and 3xTg AD mice at 3- (WT, $n=3 ; 3 x \operatorname{~AD,~} n=4), 6-(W T, n=3 ; 3 x T g A D, n=4), 12-(W T, n=4$; $3 \times \operatorname{Tg} \mathrm{AD}, \mathrm{n}=3$ ), and 24- (WT, $\mathrm{n}=4 ; 3 \times \mathrm{Tg} \mathrm{AD}, \mathrm{n}=4$ ) months of age. To compare means, significant 2-way ANOVAs were probed for effects of Genotype with planned student's ttest at each age $(*, \mathrm{p}<0.05 ; * *, \mathrm{p}<0.01 ; * * *, \mathrm{p}<0.001)$. 
occurs early, at 3-months of age (Figure 6.5), prior to the development of A $\beta$-plaques in $3 \times \mathrm{Tg}$ AD mice (Oddo et al. 2003a; Oddo et al. 2003b; Whitesall et al. 2004). Morphometry of the vasculature at the age of 3-months indicated that the earliest changes to the angioarchitecture consisted of a significantly reduced number of vessel segments in the CA1 $(t(8)=2.65, p=$ $0.01, t$-test $)$ and CA3 $(t(8)=2.05, p=0.005, t$-test $)$ hippocampal subfield(s) and a significantly reduced number of vessel-to-vessel junctions in the CA1 $(t(8)=2.77, p=0.01, t$-test $), \mathrm{CA} 3$ $(t(8)=2.32, p=0.02, t$-test $)$, and DG $(t(8)=2.25, p=0.05, t$-test $)$ subfield(s) (Figure 6.5AH). However, these differences diminished by 6-months and throughout age, whereas the total volume occupied by all vessels (Figure 6.5I-L) and the total surface area of all vessels (Figure 6.5M-P) significantly reduced, while the intervessel distance (Figure 6.5Q-T) significantly increased by 6-months and progressed with age. Furthermore, we found that the angioarchitecture of the ENT CTX to be weakly affected with age in 3xTg AD mice. Thus, we interpret these measurements to suggest that early deficits in vessel branching occur before the onset of age-associated progression of vessel loss at 6-months of age.

\section{Age and disease-related changes specific to vessel hierarchy occur in the hippocampus of 3xTg AD mice}

We next sought to extrapolate on the differences obtained by morphometric and geometric analysis of the vasculature to determine if these genotypic differences were a result of changes to vessels of a specific hierarchy. Therefore, by mapping the total length of vessels as a function of average vessel diameter, we searched for genotypic differences in the summated length of vessels per vessel diameter. We observed in 3xTg AD mice at 3-months, a major deficit of microvessels in the CA1 (Figure 6.6C), CA3 (Figure 6.6D), and DG 

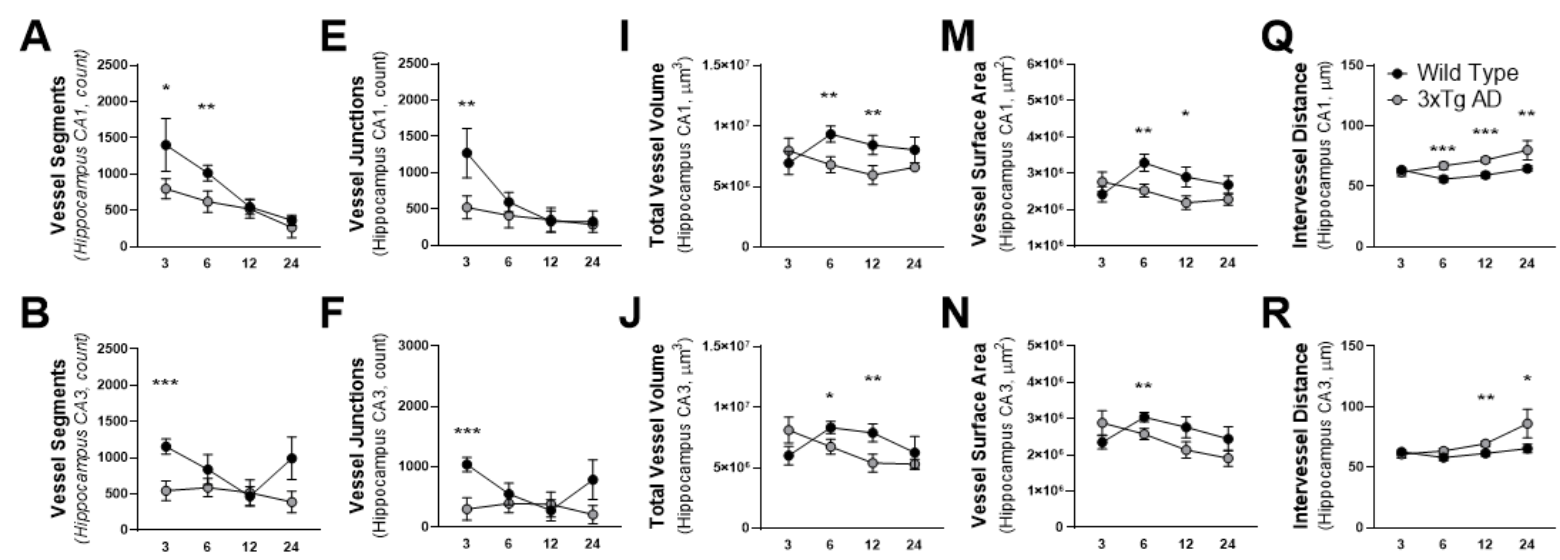

$\mathbf{R}$
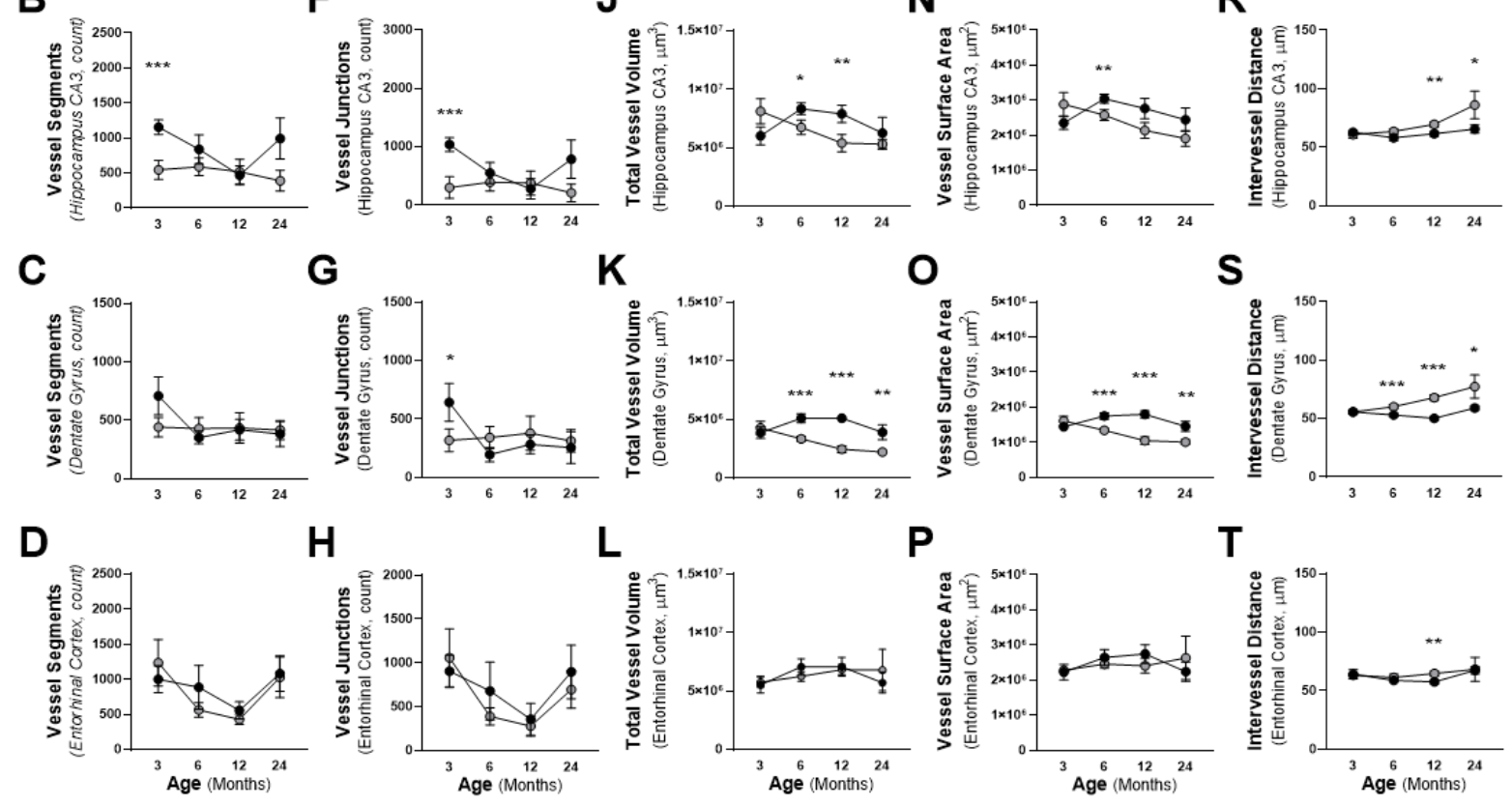

Figure 6.5. Vascular network analysis of the hippocampus indicates early and agedependent changes to the vasculature in 3xTg AD mice. Bar graph(s) (mean $\pm \mathrm{SEM}$ ) depicting (A-D) number of vessel segments, (E-H) number of vessel junctions, (I-L) total vascular volume, (M-P) total vascular surface area, and (Q-T) intervessel distance from 3(WT, $\mathrm{n}=4$; 3xTg AD, $\mathrm{n}=5$ ), 6- (WT, $\mathrm{n}=7$; 3xTg AD, $\mathrm{n}=5$ ), 12- (WT, $\mathrm{n}=5 ; 3 \mathrm{xTg} \mathrm{AD}$, $\mathrm{n}=5$ ), and 24- (WT, $\mathrm{n}=6$; $3 \times \mathrm{Tg} \mathrm{AD}, \mathrm{n}=3$ ) month $\mathrm{WT}$ and $3 \times \mathrm{Tg}$ AD mice. To compare means, significant 2-way ANOVAs within each brain region were probed for effects of Genotype with planned student's t-test at each age $\left(*, \mathrm{p}<0.05 ;{ }^{* *}, \mathrm{p}<0.01 ; * * *, \mathrm{p}<\right.$ $0.001)$. 
(Figure 6.6G) subfield(s) but not in the ENT CTX (Figure 6.6H). By 6-months, microvascular deficits worsened in the CA1 (Figure 6.6C) and persisted in the CA3 (Figure 6.6D) subregion(s) in 3xTg AD mice. In contrast, vessels with diameters larger than capillary caliber were considerably elevated in the CA1 (Figure 6.6C), CA3 (Figure 6.6D), and DG (Figure 6.6G) in $\mathrm{AD}$ mice at 3-months, then became concordant between genotypes in the CA1 and CA3 but not DG subfield(s) by 6-months of age. By this time, microvessels begin to decline and larger vessels increase in the ENT CTX in 3xTg AD mice (Figure 6.6H).

Although, capillary deficits in the CA1, CA3, and DG seem to be most severe at 3- and 6 -months of age, the deficits persist throughout age in 3xTg AD mice. Peculiar to us, is our observation of increased length of larger vessels that were seemingly transitorily affected with age in all of the measured hippocampal subregions. Nonetheless, since the data revealed profound changes to microvessels and only minor transitory changes to larger vessels we conclude that in the hippocampus of 3xTg AD mice, microvessels are the major population of vessels affected at both early and late stages of the disease progression and that these vessels account for many of the genotypic differences of the vascular network we observed via morphometric and geometric analyses.

\section{Changes to the cortical vasculature in 3xTg AD mice is age-associated and region- dependent}

We next examined the cortical vasculature via morphometric and geometric analyses to determine the time-course of the onset and progression of vascular disruption. In order to make these assessments, we measured the intensity of vessel branching, regional vascularization, and network efficacy in the MO PFC, SS CTX, and CC in 3xTg AD and WT 


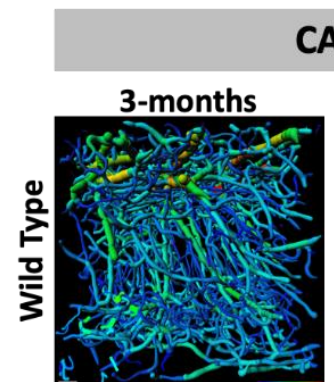

\section{CA1}
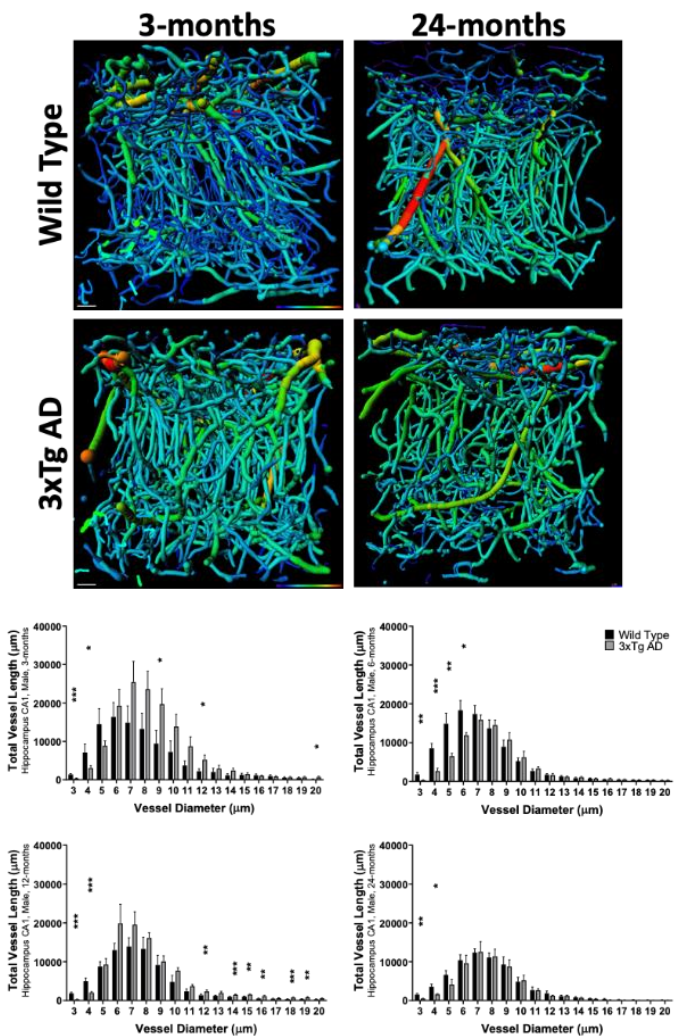

\section{Dentate Gyrus}
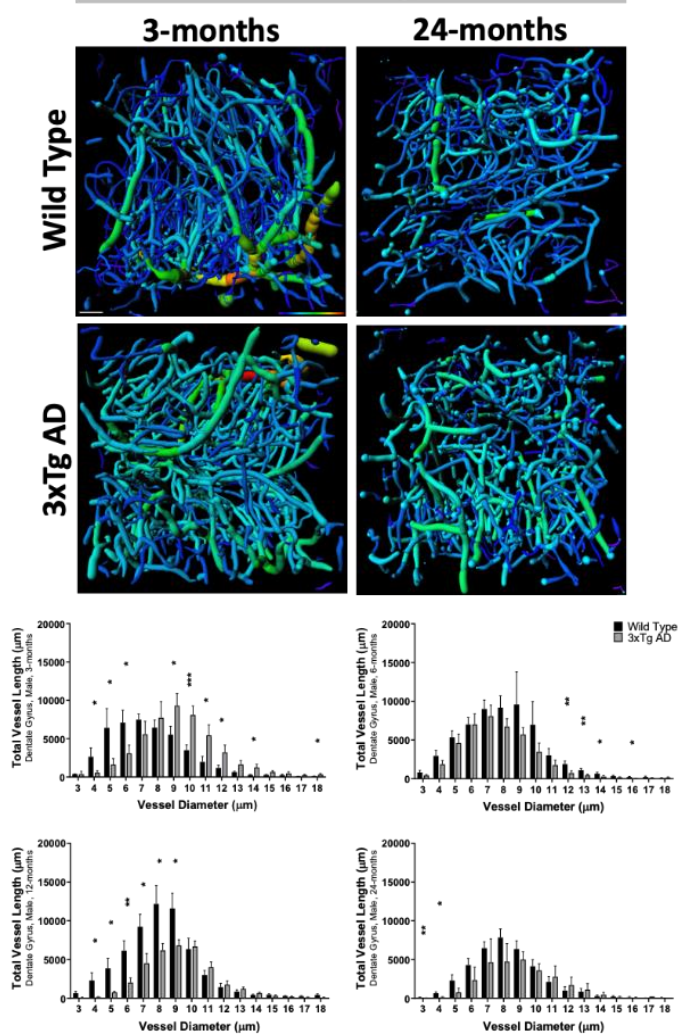

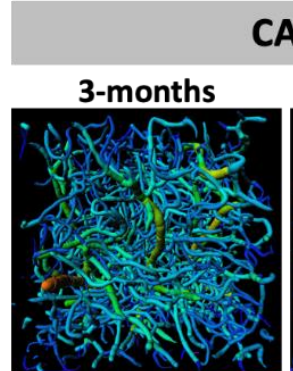

\section{CA3}
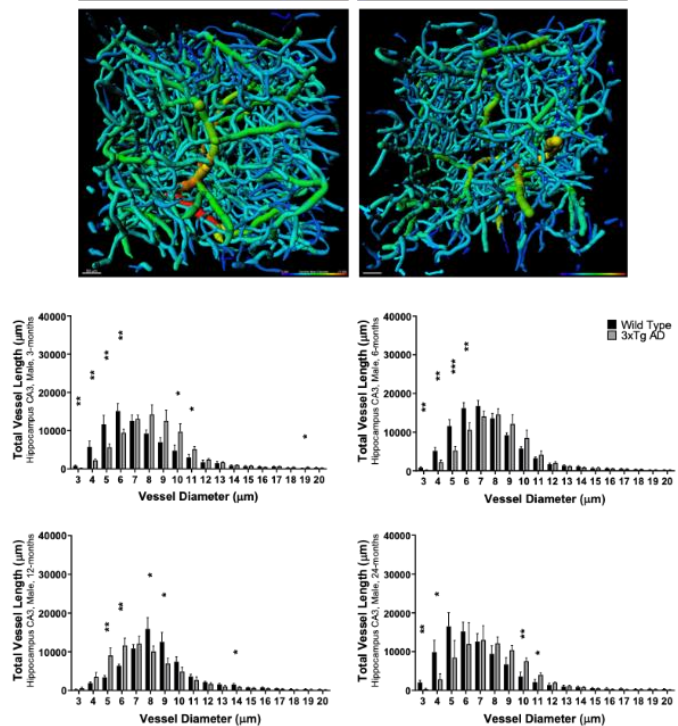

Entorhinal Cortex
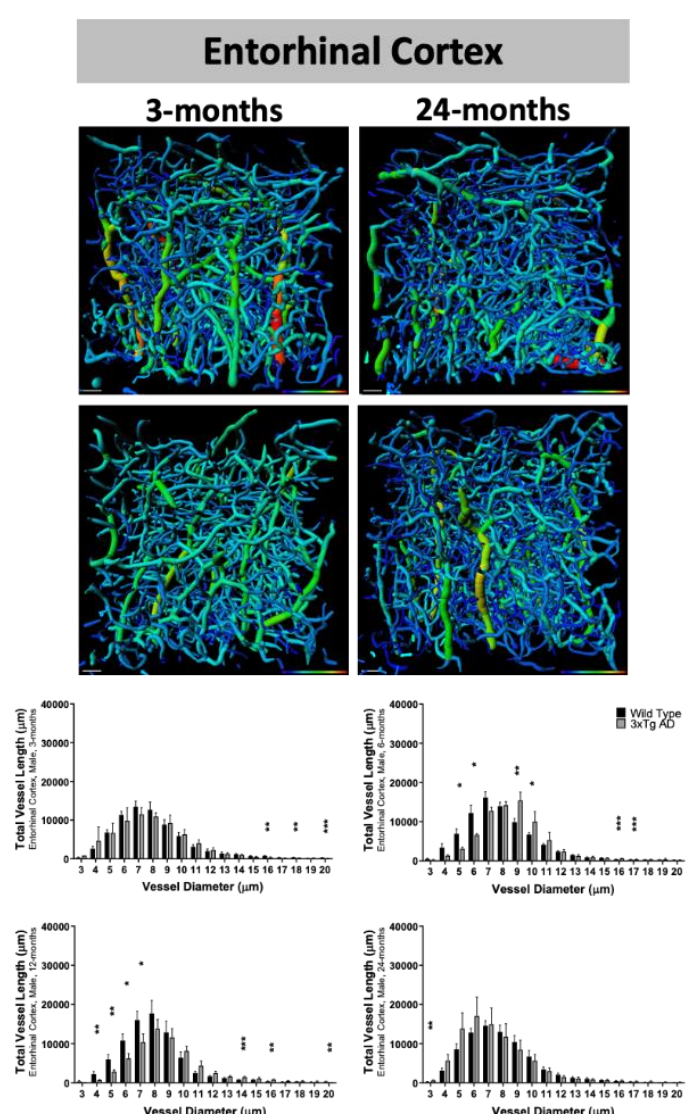
Figure 6.6. Microvascular degeneration in the hippocampus occurs early and progresses with age in a hippocampal subregion specific manner. Reconstructed vascular network, color-coded by vessel diameter from hippocampal subregions (A) CA1, (B) CA3, (C) DG, (D) and entorhinal cortex from WT and 3xTg AD mice. Grouped bar $\operatorname{graph}(\mathrm{s})$ (mean $\pm \mathrm{SEM}$ ) depicting the total length of vessels as a function of average vessel diameter in hippocampal subregion(s) (E-H) CA1, (I-L) CA3, (M-P) DG, and (Q-T) entorhinal cortex from 3- (WT, $n=4 ; 3 x T g A D, n=5), 6-(W T, n=7 ; 3 x T g A D, n=5)$, 12- (WT, $\mathrm{n}=5 ; 3 \times \operatorname{Tg}$ AD, $\mathrm{n}=5)$, and 24- (WT, $\mathrm{n}=5$; 3xTg AD, $\mathrm{n}=3$ ) month old WT and 3xTg AD mice. To compare means, significant 2-way ANOVAs within each age group and in each region were probed for effects of Genotype with planned student's t-test at each vessel diameter $(*, \mathrm{p}<0.05 ; * *, \mathrm{p}<0.01 ; * * *, \mathrm{p}<0.001)$. 
mice. Deposits of extracellular A $\beta$ in the MO PFC begin to appear at 6-months of age in layers 4 and 5 and becomes severe by 12-months (Oddo et al. 2003b; Oddo et al. 2003a). Similar to the neuropathological progression in 3xTg AD mice, deposition of $\mathrm{A} \beta$ in human patients with $\mathrm{AD}$ usually begin in the cortex and later in the hippocampus while tangles begin to develop in the limbic regions and progress to the cortex (Mesulam 1999). Our analyses revealed that the vascular network of the $\mathrm{CC}$ was the only cortical region to be affected at 3-months of age in 3xTg AD mice. Similar to that observed in the hippocampus, the number of vessel segments (Figure 6.7C) and number of vessel junctions (Figure 6.7F) were significantly reduced in 3xTg $\mathrm{AD}$ mice compared to age-matched WT mice. However, these genotypic differences diminished by 6-months and throughout age in the $\mathrm{CC}$. We found no differences to the number of vessel segments and the number of junctions in the MO PFC (Figure 6.7A) and SS CTX (Figure 6.7B) at 3-months, however by 6-months there were significantly fewer vessel segments and junctions in the MO PFC but not in the SS CTX of 3xTg AD mice. Compromised vascular volume was initially observed at 6-months in the MO PFC (Figure 6.7G) and CC (Figure 6.7I) and again at 12-months of age but only in the CC. In fact, at 6-months of age, the total surface area of all vessels and the intervessel distance was significantly reduced in all cortical regions evaluated. At 12-months of age, the surface area of all vessels in the MO PFC (Figure 6.7J) and CC (Figure 6.7L) was significantly reduced in 3xTg AD mice. Similarly, the intervessel distance at the MO PFC (Figure 6.7M), SS CTX (Figure 6.7N), and CC (Figure 6.7O) of 3xTg AD mice was significantly higher at 6-months, an effect that largely persisted at later ages. 

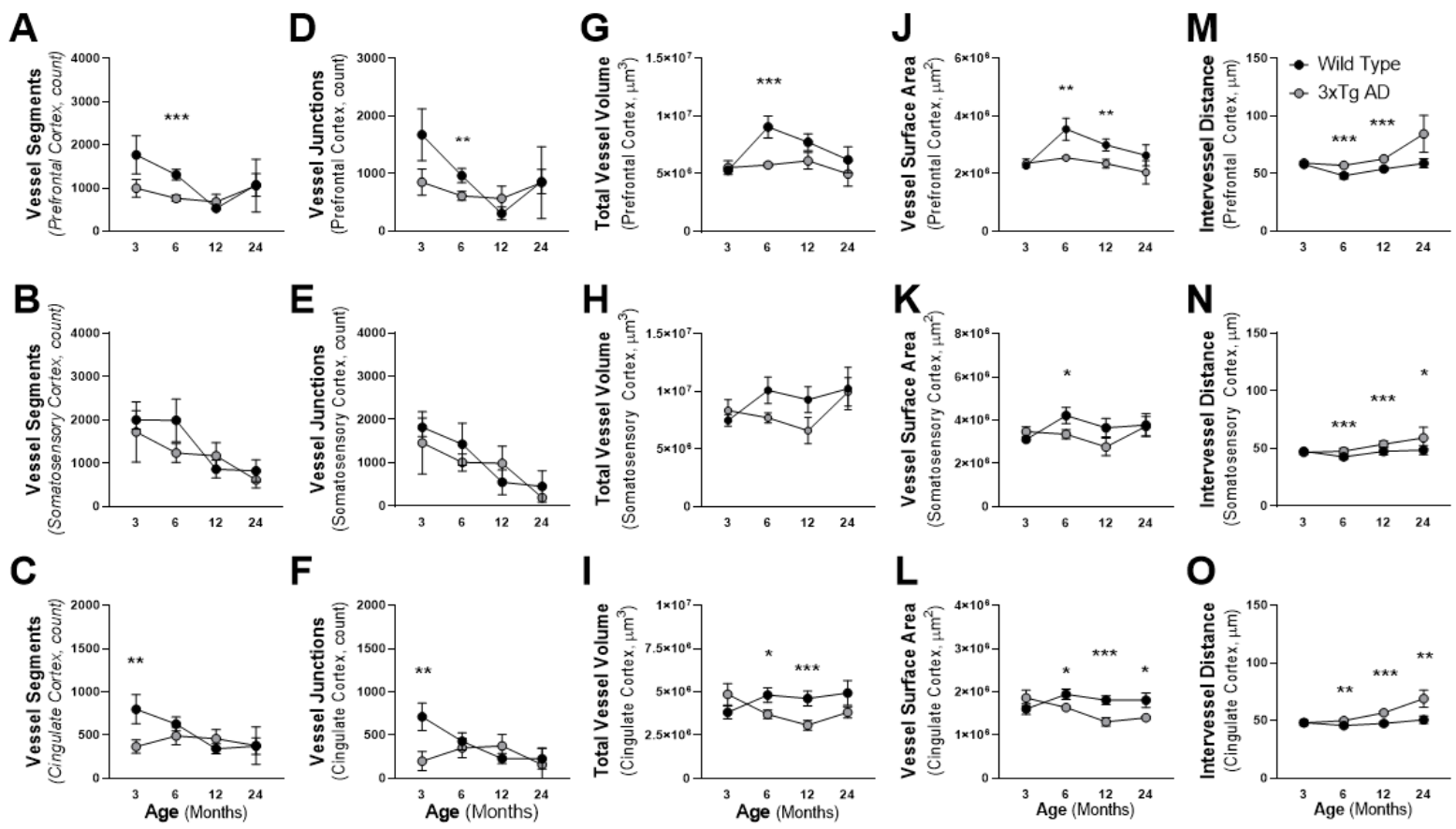

Figure 6.7. Vascular network analysis of the cortex indicates early and age-dependent changes to the vasculature in 3xTg AD mice. Bar graph(s) (mean \pm SEM) depicting (AC) number of vessel segments, (D-F) number of vessel junctions, (G-I) total vascular volume, (J-L) total vascular surface area, and (M-O) intervessel distance from 3- (WT, $\mathrm{n}=$ 4; 3xTg AD, $n=5$ ), 6- (WT, $n=7 ; 3 x \operatorname{Tg} A D, n=5), 12-(\mathrm{WT}, \mathrm{n}=5 ; 3 \times \operatorname{Tg} \mathrm{AD}, \mathrm{n}=5)$, and 24- (WT, $\mathrm{n}=5$; $3 \times \mathrm{Tg} \mathrm{AD}, \mathrm{n}=4$ ) month $\mathrm{WT}$ and $3 \times \mathrm{Tg} \mathrm{AD}$ mice. To compare means, significant 2-way ANOVAs within each brain region were probed for effects of Genotype with planned student's t-test at each age $\left(*, \mathrm{p}<0.05 ; *^{*}, \mathrm{p}<0.01 ; * *, \mathrm{p}<0.001\right)$. 


\section{Early deficits of cortical microvessels progress with age in 3xTg AD mice}

We next extrapolated on these differences by evaluating the changes that occur to vessels of specific hierarchy. Using local thickness maps of vessels, we calculated the total length of vessels as a function of average vessel diameter. We found that the earliest changes to the vasculature occur in the SS CTX and CC at the age of 3-months. In both of these regions, 3xTg AD mice demonstrated a deficit of microvessels that had an average diameter less than $5 \mu \mathrm{m}$ and a significant increase of vessels with a diameter greater than $7 \mu \mathrm{m}$ (Figure 6.8). At 6-months, microvessels with an average diameter of 3 and $4 \mu \mathrm{m}$ were significantly reduced in all three cortical regions (Figure 6.8A-C). However, at this age, larger vessels in the MO PFC and $\mathrm{CC}$ were reduced in 3xTg AD compared to WT mice (Figure 6.8). At 12-months of age, microvessel deficits were observed only in the SS CTX and MO PFC (Figure 6.8). Interestingly, larger vessels were increased in the MO PFC while reduced in the SS CTX and $\mathrm{CC}$ in $3 \mathrm{xTg} \mathrm{AD}$ mice. By 24-months of age, microvessel deficits were observed only in the MO PFC of 3xTg AD mice. Whereas, deficits of larger vessels were observed in the MO PFC and $\mathrm{CC}$ of $3 \times \mathrm{Tg} \mathrm{AD}$ mice at 24-months of age (Figure 6.8).

\section{Discussion}

Although numerous neuroimaging studies in patients with $\mathrm{AD}$ confirm a reduction in $\mathrm{CBF}$ during early and late stages of $\mathrm{AD}$, it remains unclear whether cerebrovascular hypoperfusion is a cause or consequence of AD. Pathological changes to the structure of the microvasculature have been shown to be more prominent in demented than in non-demented individuals (Brown et al. 2009). Further evaluation of the pathological changes that occur to 

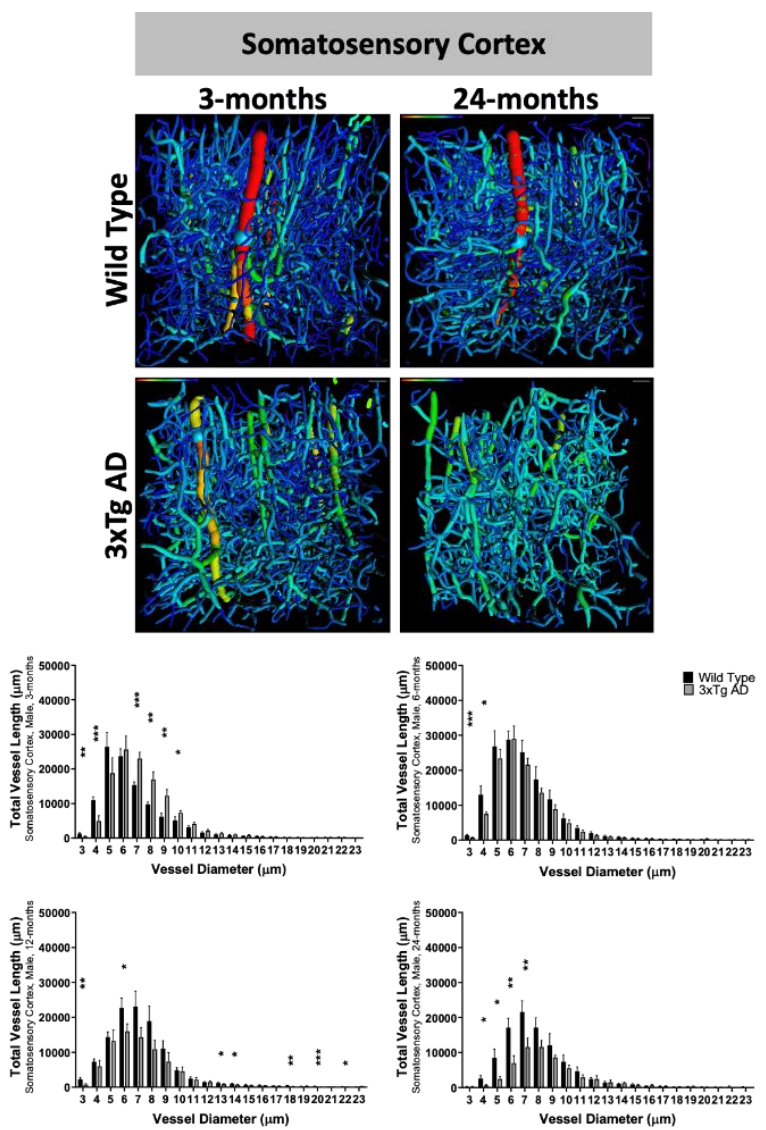

\section{Cingulate Cortex}

3-months
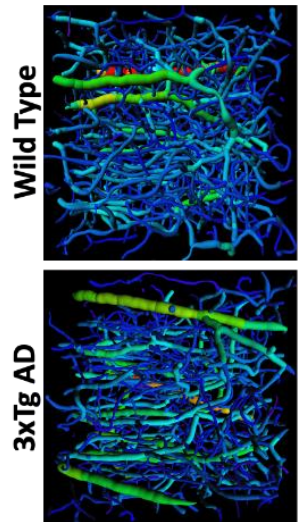

24-months
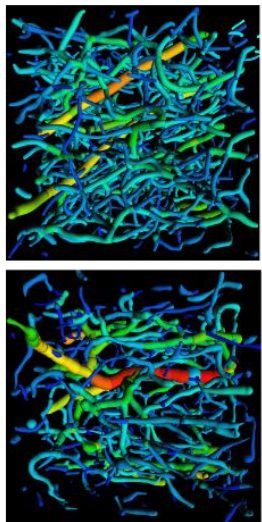
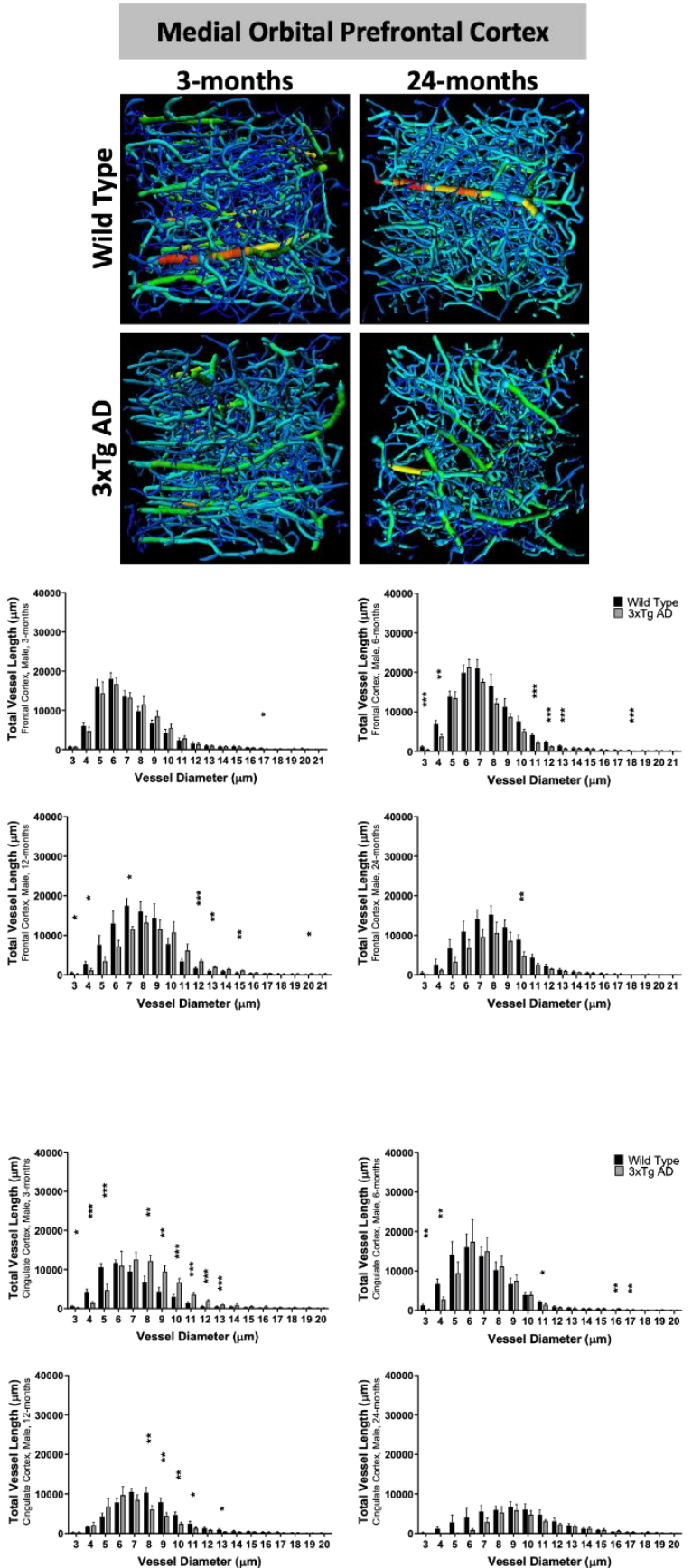
Figure 6.8. Microvascular degeneration in the cortex occurs early and progresses with age in a subregion specific manner. Reconstructed vascular network color-coded by vessel diameter from hippocampal subregions (A) somatosensory cortex (SS CTX), (B) medial orbital prefrontal cortex (MO PFC), and (C) cingulate cortex from WT and 3xTg AD mice. Grouped bar graph(s) (mean \pm SEM) depicting the total length of vessels as a function of average vessel diameter in cortical subregion(s) (D-G) SS CTX, (H-K) MO PFC, and (L-O) cingulate cortex from 3- (WT, $\mathrm{n}=4$; 3xTg AD, $\mathrm{n}=5)$, 6- $(\mathrm{WT}, \mathrm{n}=7$; 3xTg $\mathrm{AD}, \mathrm{n}=5), 12-(\mathrm{WT}, \mathrm{n}=5 ; 3 \times \mathrm{Tg} \mathrm{AD}, \mathrm{n}=5)$, and 24- (WT, $\mathrm{n}=5 ; 3 \times \mathrm{Tg}$ AD, $\mathrm{n}=4)$ month old WT and 3xTg AD mice. To compare means, significant 2-way ANOVAs within each age group and in each region were probed for effects of Genotype with planned student's ttest at each vessel diameter $(*, \mathrm{p}<0.05 ; * *, \mathrm{p}<0.01 ; * * *, \mathrm{p}<0.001)$. 
the cerebrovasculature during $\mathrm{AD}$ offers a promising approach to identify useful biomarkers for the detection of $\mathrm{AD}$ at early stages.

In the present study, our goal was to characterize the changes to the cerebrovasculature in $3 \times \mathrm{Tg} \mathrm{AD}$ mice by establishing the time-course for vascular degeneration with age and the progression of $\mathrm{AD}$. We hypothesized that the degeneration of the microvasculature is a major component of the pathophysiology of AD. We addressed this hypothesis by evaluating the microvasculature of the whole brain and in key brain regions with age and the progression of $\mathrm{AD}$ in $3 \mathrm{xTg} \mathrm{AD}$ mice.

Vascular risk factors for hypoperfusion can cause hemodynamic changes to the brain microvasculature that can result in cognitive impairment by preventing optimal delivery of oxygen and glucose to the brain (Duron and Hanon 2008). Furthermore, previous studies conducted with $\mathrm{AD}$ patients suggest that hypoxia increases the levels of $\mathrm{A} \beta$ in the vasculature of the brain (Peers et al. 2009). It is believed that $\mathrm{A} \beta$ exerts its toxicity to the vasculature by dysregulating intracellular calcium homeostasis, which can lead to the dysfunction of many cellular processes (Quintana et al. 2020).

Reconstruction of cerebrovascular corrosion casts in three-dimension demonstrated visual evidence of chronic cerebrovascular injury in 3xTg AD mice including, tortuous vessels in the cortex (Figure 6.1B and C) and aneurysms of capillaries and arterioles (Figure 6.1D-F). Progressive weakening of the structural elements of the vascular wall is responsible for both the formation of aneurysms and tortuosity vessels (Dobrin et al. 1988). Elastin is an important extracellular matrix protein of the vessel wall and its degradation can compromise the structural integrity of the vascular wall (Dobrin and Canfield 1984). Elastin deficiency has been implicated in the formation of tortuous vessels in patients with arterial tortuosity 
syndrome and in transgenic mice (Taarnhøj et al. 2008; Nakamura et al. 2002; Coucke et al. 2006; Yanagisawa et al. 2002; Carta et al. 2009). Elastolytic enzymes have been noted to be elevated in the aneurysmal wall, including neutrophil elastase and members of the matrix metalloprotease (MMP) class. It is likely that the deposition of $A \beta$ on the wall of cerebral blood vessels causes damage to the extracellular matrix and degeneration of the vessel wall. Noteworthy, vessel stress can cause the activation of vascular smooth muscle cells (vSMC) resulting in a phenotype shift from a contractile state into a biosynthetic state. Activated vSMC directly participate in the destabilization of the vascular wall via the production of matrixdegrading proteases such as MMPs. Activation of vSMC may provide a potential mechanism for the $A \beta$-induced damage to cerebral blood vessels and to the formation of aneurysms and tortuous vessels.

Cerebrovascular hypoperfusion causes metabolic stress and degeneration of the brain parenchyma (Quintana et al. 2018). We found that at 6-months, CBF begins to decline progressively with age in $3 \mathrm{xTg} \mathrm{AD}$ mice (Figure 6.2). As cerebrovascular hypoperfusion progresses, reactive oxygen species (ROS) are produced and accumulate within mitochondria (Halestrap and Pasdois 2009; Clanton 2007). Concomitantly, hypoperfusion results in ATP depletion, causing high intracellular $\mathrm{Na}^{+}$concentration, which compromises the $\mathrm{Na}^{+} / \mathrm{Ca}^{+}$ antiporter resulting in the accumulation of intracellular $\mathrm{Ca}^{+}$(Halestrap and Pasdois 2009). Increased intracellular $\mathrm{Ca}^{+}$concentration together with elevated levels of mitochondrial ROS induce the formation of the mitochondrial membrane permeability transition pore (mPTP), facilitating the pathway for necrotic cell death (Halestrap 2010; Loor et al. 2011).

Geometric analysis of the angioarchitecture revealed early changes to the cerebrovasculature that indicated morphological response to chronic injury. Interestingly, 
these early changes occurred at 3-months of age, and included alterations to the average vessel diameter, intervessel distance, and pathway redundancy of the vascular network, suggesting an alteration to the network composition. This was in contrast to the changes to the angioarchitecture that occurred following 6-months of age. In 3xTg AD mice at later ages, whole brain analyses of the angioarchitecture demonstrated changes that indicated degeneration of the cerebrovasculature. These changes included a decline in number of vessel segments that occurred with reduced vascular volume, vessel surface area and a greater distance between vessels. Along with these degenerative changes to the angioarchitecture, late age was also associated with reduced number of vessel-to-vessel junctions and compromised network complexity in 3xTg AD mice.

Consistent with the literature, whole brain analyses of vessel volume as a function of average vessel diameter revealed smaller vessels to be most affected by age in 3xTg AD mice, indicating that the majority of the changes to the angioarchitecture are accounted for by changes to the microvasculature.

When we assessed the region specific changes to the hippocampal and cortical vasculature with age, we found early differences to the number of vessel segments and vesselto-vessel junctions at 3-months of age. The most severely affected region of the hippocampus was the CA1, DG, CA3, and then ENT CTX, in that order. Similar to our whole brain analyses, changes to the hippocampal vasculature in $3 \times \mathrm{Tg} \mathrm{AD}$ mice indicated network adaptations to chronic injury that occurs early and progresses with age. Hippocampal vasculature began to demonstrate evidence of vascular degeneration at 6-month that progressed with age in $3 \times \mathrm{Tg}$ $\mathrm{AD}$ mice. The hippocampus is among the first region of the brain to accumulate $\mathrm{A} \beta$ plaque in patients and mice with Alzheimer's disease. In 3xTg AD mice, the hippocampus develops 
sparse $\mathrm{A} \beta$ plaques by 6-months of age and by 12-months plaque deposition is dense (Oddo $e t$ al. 2003a). We found that the major contribution to these changes were via alterations to the microvasculature. Our analysis of the distribution of vessels by hierarchy as a function of average vessel diameter indicated that changes to the microvascular network occurred with age and differed between genotype. These data indicate that vascular damage in the hippocampus occurs before the onset of plaque deposition in 3xTg AD mice.

Hypoperfusion of the frontal cortex is implicated in causing the unawareness of cognitive deficits in patients with AD (Amanzio et al. 2011). Likewise, hypoperfusion of the cingulate cortex is associated with the impaired orientation for time in AD patients (Yamashita et al. 2019). Region specific analyses of the cortical vasculature indicated it to be less affected than regions of the hippocampus. The deposition of $A \beta$ plaques in cortical regions is first detected at 12-months of age in 3xTg AD mice (Belfiore et al. 2019). We found the angioarchitecture of the MO PFC to be the most severely affected cortical region. At 3-months, the vasculature of the CC and not the MO PFC and SS CTX, demonstrated a reduced number of vessel segments and a lower count of vessel-to-vessel junctions. At 6-months of age, the MO PFC contained a reduced number of segments, vessel junctions, total vessel volume, surface area, and intervessel distance. Whereas, the angioarchitecture of the SS CTX and CC provided a reduced surface area of vessels and a greater distance between vessels relative to age-matched WT mice. Interestingly, at 12- and 24-months of age, an increased intervessel distance was the only measured parameter to be affected and in all cortical regions assessed.

Overall, our data demonstrate that the cerebrovasculature of $3 \times \mathrm{Tg} A D$ mice is affected early during aging, and is characterized by morphological and structural adaptations that are likely caused by chronic injury to vessels. These data indicated that alteration to the 
angioarchitecture occurs before the onset of plaque development. Once plaque deposition occurs, changes to the angioarchitecture begin to indicate structural changes that occur via vascular degeneration. These data suggest that changes to the vasculature that occur early during $\mathrm{AD}$ may progress, resulting in cerebrovascular degeneration, initiating the onset of clinically detectable $\mathrm{AD}$ in patients.

\section{References}

Amanzio M., Torta D. M. E., Sacco K., Cauda F., D’Agata F., Duca S., Leotta D., Palermo S., Geminiani G. C. (2011) Unawareness of deficits in Alzheimer's disease: role of the cingulate cortex. Brain 134, 1061-1076.

Bailey T. L., Rivara C. B., Rocher A. B., Hof P. R. (2004) The nature and effects of cortical microvascular pathology in aging and Alzheimer's disease. Neurol. Res. 26, 573-578.

Belfiore R., Rodin A., Ferreira E., Velazquez R., Branca C., Caccamo A., Oddo S. (2019) Temporal and regional progression of Alzheimer's disease-like pathology in 3xTg-AD mice. Aging Cell 18, e12873.

Billings L. M., Oddo S., Green K. N., McGaugh J. L., LaFerla F. M. (2005) Intraneuronal Abeta causes the onset of early Alzheimer's disease-related cognitive deficits in transgenic mice. Neuron 45, 675-688.

Brown W. R., Moody D. M., Thore C. R., Anstrom J. A., Challa V. R. (2009) Microvascular changes in the white mater in dementia. J. Neurol. Sci. 283, 28-31.

Brundel M., Bresser J. de, Dillen J. J. van, Kappelle L. J., Biessels G. J. (2012a) Cerebral microinfarcts: a systematic review of neuropathological studies. J. Cereb. Blood Flow Metab. 32, 425-436.

Brundel M., Heringa S. M., Bresser J. de, Koek H. L., Zwanenburg J. J. M., Jaap Kappelle L., Luijten P. R., Biessels G. J. (2012b) High prevalence of cerebral microbleeds at 7Tesla MRI in patients with early Alzheimer's disease. J. Alzheimers. Dis. 31, 259-263. 
Carta L., Wagenseil J. E., Knutsen R. H., Mariko B., Faury G., Davis E. C., Starcher B., Mecham R. P., Ramirez F. (2009) Discrete contributions of elastic fiber components to arterial development and mechanical compliance. Arterioscler. Thromb. Vasc. Biol. 29, 2083-2089.

Castellani R. J., Rolston R. K., Smith M. A. (2010) Alzheimer disease. Dis. Mon. 56, 484546.

Chi N.-F., Chien L.-N., Ku H.-L., Hu C.-J., Chiou H.-Y. (2013) Alzheimer disease and risk of stroke: a population-based cohort study. Neurology $\mathbf{8 0}, 705-711$.

Clanton T. L. (2007) Hypoxia-induced reactive oxygen species formation in skeletal muscle. J. Appl. Physiol. 102, 2379-2388.

Coucke P. J., Willaert A., Wessels M. W., Callewaert B., Zoppi N., Backer J. De, Fox J. E., et al. (2006) Mutations in the facilitative glucose transporter GLUT10 alter angiogenesis and cause arterial tortuosity syndrome. Nat. Genet. 38, 452-457.

Daulatzai M. A. (2017) Cerebral hypoperfusion and glucose hypometabolism: Key pathophysiological modulators promote neurodegeneration, cognitive impairment, and Alzheimer's disease. J. Neurosci. Res. 95, 943-972.

DeSimone C. V., Graff-Radford J., El-Harasis M. A., Rabinstein A. A., Asirvatham S. J., Holmes D. R. (2017) Cerebral Amyloid Angiopathy: Diagnosis, Clinical Implications, and Management Strategies in Atrial Fibrillation. J. Am. Coll. Cardiol. 70, 1173-1182.

Dobrin P. B., Canfield T. R. (1984) Elastase, collagenase, and the biaxial elastic properties of dog carotid artery. Am. J. Physiol. 247, H124-31.

Dobrin P. B., Schwarcz T. H., Baker W. H. (1988) Mechanisms of arterial and aneurysmal tortuosity. Surgery 104, 568-571.

Dong S., Maniar S., Manole M. D., Sun D. (2018) Cerebral Hypoperfusion and Other Shared Brain Pathologies in Ischemic Stroke and Alzheimer's Disease. Transl. Stroke Res. 9, $238-250$.

Duron E., Hanon O. (2008) Vascular risk factors, cognitive decline, and dementia. Vasc. Health Risk Manag. 4, 363-381. 
España J., Giménez-Llort L., Valero J., Miñano A., Rábano A., Rodriguez-Alvarez J., LaFerla F. M., Saura C. A. (2010) Intraneuronal beta-amyloid accumulation in the amygdala enhances fear and anxiety in Alzheimer's disease transgenic mice. Biol. Psychiatry 67, 513-521.

Farkas E., Jong G. I. De, Apro E., Keuker J. I., Luiten P. G. (2001) Calcium antagonists decrease capillary wall damage in aging hypertensive rat brain. Neurobiol. Aging 22 , 299-309.

Fischer V. W., Siddiqi A., Yusufaly Y. (1990) Altered angioarchitecture in selected areas of brains with Alzheimer's disease. Acta Neuropathol. 79, 672-679.

Giménez-Llort L., Blázquez G., Cañete T., Johansson B., Oddo S., Tobeña A., LaFerla F. M., Fernández-Teruel A. (2007) Modeling behavioral and neuronal symptoms of Alzheimer's disease in mice: a role for intraneuronal amyloid. Neurosci. Biobehav. Rev. 31, $125-147$.

Göttler J., Preibisch C., Riederer I., Pasquini L., Alexopoulos P., Bohn K. P., Yakushev I., et al. (2019) Reduced blood oxygenation level dependent connectivity is related to hypoperfusion in Alzheimer's disease. J. Cereb. bloodflow Metab. Off. J. Int. Soc. Cereb. Blood Flow Metab. 39, 1314-1325.

Gould I. G., Tsai P., Kleinfeld D., Linninger A. (2017) The capillary bed offers the largest hemodynamic resistance to the cortical blood supply. J. Cereb. Blood Flow Metab. 37, $52-68$.

Gross L. (2006) Redundancy in cortical surface vessels supports persistent blood flow. PLoS Biol. 4, e43.

Halestrap A. P. (2010) A pore way to die: the role of mitochondria in reperfusion injury and cardioprotection. England.

Halestrap A. P., Pasdois P. (2009) The role of the mitochondrial permeability transition pore in heart disease. Biochim. Biophys. Acta 1787, 1402-1415.

Han B. H., Zhou M.-L., Johnson A. W., Singh I., Liao F., Vellimana A. K., Nelson J. W., et al. (2015) Contribution of reactive oxygen species to cerebral amyloid angiopathy, vasomotor dysfunction, and microhemorrhage in aged Tg2576 mice. Proc. Natl. Acad. 
Sci. U. S. A. 112, E881-90.

Iadecola C. (2004) Neurovascular regulation in the normal brain and in Alzheimer's disease. Nat. Rev. Neurosci. 5, 347-360.

Iadecola C., Zhang F., Niwa K., Eckman C., Turner S. K., Fischer E., Younkin S., Borchelt D. R., Hsiao K. K., Carlson G. A. (1999) SOD1 rescues cerebral endothelial dysfunction in mice overexpressing amyloid precursor protein. Nat. Neurosci. 2, 157-161.

Janson C. G. (2015) AD and CAA: Independent risk factors for dementia. Sci. Transl. Med. 7, 318ec214-318ec214.

Jellinger K. A. (2010) Prevalence and impact of cerebrovascular lesions in Alzheimer and lewy body diseases. Neurodegener. Dis. 7, 112-115.

Kalaria R. N. (2010) Vascular basis for brain degeneration: faltering controls and risk factors for dementia. Nutr. Rev. 68 Suppl 2, S74-87.

Kalaria R. N., Hedera P. (1995) Differential degeneration of the cerebral microvasculature in Alzheimer's disease. Neuroreport 6, 477-480.

la Torre J. C. de (2004) Alzheimer's disease is a vasocognopathy: a new term to describe its nature. Neurol. Res. 26, 517-524.

la Torre J. C. de (2010) Vascular risk factor detection and control may prevent Alzheimer's disease. Ageing Res. Rev. 9, 218-225.

Lein E. S., Hawrylycz M. J., Ao N., Ayres M., Bensinger A., Bernard A., Boe A. F., et al. (2007) Genome-wide atlas of gene expression in the adult mouse brain. Nature $\mathbf{4 4 5}$, $168-176$.

Loor G., Kondapalli J., Iwase H., Chandel N. S., Waypa G. B., Guzy R. D., Hoek T. L. Vanden, Schumacker P. T. (2011) Mitochondrial oxidant stress triggers cell death in simulated ischemia-reperfusion. Biochim. Biophys. Acta 1813, 1382-1394.

Love S., Miners J. S. (2016) Cerebral Hypoperfusion and the Energy Deficit in Alzheimer's Disease. Brain Pathol. 26, 607-617.

Marrif H., Juurlink B. H. J. (1999) Astrocytes respond to hypoxia by increasing glycolytic capacity. J. Neurosci. Res. 57, 255-260. 
Mesulam M. M. (1999) Neuroplasticity failure in Alzheimer's disease: bridging the gap between plaques and tangles. Neuron 24, 521-529.

Nakamura T., Lozano P. R., Ikeda Y., Iwanaga Y., Hinek A., Minamisawa S., Cheng C.-F., et al. (2002) Fibulin-5/DANCE is essential for elastogenesis in vivo. Nature 415, 171175.

Niwa K., Younkin L., Ebeling C., Turner S. K., Westaway D., Younkin S., Ashe K. H., et al. (2000) Abeta 1-40-related reduction in functional hyperemia in mouse neocortex during somatosensory activation. Proc. Natl. Acad. Sci. U. S. A. 97, 9735-40.

Nostrand W. E. Van, Melchor J. P., Cho H. S., Greenberg S. M., Rebeck G. W. (2001) Pathogenic effects of D23N Iowa mutant amyloid beta -protein. J. Biol. Chem. 276, $32860-32866$.

Nyengaard J. R. (1999) Stereologic methods and their application in kidney research. J. Am. Soc. Nephrol. 10, 1100-23.

Oddo S., Caccamo A., Kitazawa M., Tseng B. P., LaFerla F. M. (2003a) Amyloid deposition precedes tangle formation in a triple transgenic model of Alzheimer's disease. Neurobiol. Aging 24, 1063-1070.

Oddo S., Caccamo A., Shepherd J. D., Murphy M. P., Golde T. E., Kayed R., Metherate R., Mattson M. P., Akbari Y., LaFerla F. M. (2003b) Triple-transgenic model of Alzheimer's disease with plaques and tangles: intracellular Abeta and synaptic dysfunction. Neuron 39, 409-421.

Peers C., Dallas M. L., Boycott H. E., Scragg J. L., Pearson H. A., Boyle J. P. (2009) Hypoxia and neurodegeneration. Ann. N. Y. Acad. Sci. 1177, 169-177.

Purandare N., Burns A. (2009) Cerebral emboli in the genesis of dementia. J. Neurol. Sci. 283, 17-20.

Querfurth H. W., LaFerla F. M. (2010) Alzheimer's Disease. N. Engl. J. Med. 362, 329-344. Quintana D. D., Garcia J. A., Anantula Y., Rellick S. L., Engler-Chiurazzi E. B., Sarkar S. N., Brown C. M., Simpkins J. W. (2020) Amyloid- $\beta$ Causes Mitochondrial Dysfunction via a Ca2+-Driven Upregulation of Oxidative Phosphorylation and Superoxide 
Production in Cerebrovascular Endothelial Cells. J. Alzheimers. Dis. 75, 119-138.

Quintana D. D., Lewis S. E., Anantula Y., Garcia J. A., Sarkar S. N., Cavendish J. Z., Brown C. M., Simpkins J. W. (2019) The cerebral angiome: High resolution MicroCT imaging of the whole brain cerebrovasculature in female and male mice. Neuroimage, 116109.

Quintana D. D., Ren X., Hu H., Engler-Chiurazzi E. B., Rellick S. L., Lewis S. E., Povroznik J. M., Simpkins J. W., Alvi M. (2018) Gradual common carotid artery occlusion as a novel model for cerebrovascular Hypoperfusion. Metab. Brain Dis. 33, 2039-2044.

Rosenberg G. A., Wallin A., Wardlaw J. M., Markus H. S., Montaner J., Wolfson L., Iadecola C., et al. (2016) Consensus statement for diagnosis of subcortical small vessel disease. J. Cereb. blood flow Metab. Off. J. Int. Soc. Cereb. Blood Flow Metab. 36, 625 .

Santos C. Y., Snyder P. J., Wu W. C., Zhang M., Echeverria A., Alber J. (2017) Pathophysiologic relationship between Alzheimer's disease, cerebrovascular disease, and cardiovascular risk: A review and synthesis. Elsevier Inc.

Sheth S. A., Liebeskind D. S. (2014) \&quot;Imaging Evaluation of Collaterals in the Brain: Physiology and Clinical Translation\&quot; Curr. Radiol. Rep. $2,29$.

Shi X., Ohta Y., Liu X., Shang J., Morihara R., Nakano Y., Feng T., et al. (2019) Chronic Cerebral Hypoperfusion Activates the Coagulation and Complement Cascades in Alzheimer's Disease Mice. Neuroscience 416, 126-136.

Taarnhøj N. C. B. B., Munch I. C., Sander B., Kessel L., Hougaard J. L., Kyvik K., Sørensen T. I. A., Larsen M. (2008) Straight versus tortuous retinal arteries in relation to blood pressure and genetics. Br. J. Ophthalmol. 92, 1055-1060.

Thomas T., Miners S., Love S. (2015) Post-mortem assessment of hypoperfusion of cerebral cortex in Alzheimer's disease and vascular dementia. Brain 138, 1059-1069.

Thomas T., Thomas G., McLendon C., Sutton T., Mullan M. (1996) B-Amyloid-mediated vasoactivity and vascular endothelial damage. Nature 380, 168-171.

Tolppanen A.-M., Lavikainen P., Solomon A., Kivipelto M., Soininen H., Hartikainen S. (2013) Incidence of stroke in people with Alzheimer disease: a national register-based 
approach. Neurology 80, 353-358.

Verclytte S., Lopes R., Lenfant P., Rollin A., Semah F., Leclerc X., Pasquier F., Delmaire C. (2016) Cerebral Hypoperfusion and Hypometabolism Detected by Arterial Spin Labeling MRI and FDG-PET in Early-Onset Alzheimer's Disease. J. Neuroimaging 26, 207-212.

Vinters H. V (1987) Cerebral amyloid angiopathy. A critical review. Stroke 18, 311-324.

Vinters H. V, Farag E. S. (2003) Amyloidosis of cerebral arteries. Adv. Neurol. 92, 105-112.

Wehrwein E. A., Joyner M. J. (2013) Regulation of blood pressure by the arterial baroreflex and autonomic nervous system. Handb. Clin. Neurol. 117, 89-102.

Whitesall S. E., Hoff J. B., Vollmer A. P., D’Alecy L. G. (2004) Comparison of simultaneous measurement of mouse systolic arterial blood pressure by radiotelemetry and tail-cuff methods. Am. J. Physiol. Heart Circ. Physiol. 286, H2408-15.

Wilde E., Aubdool A. A., Thakore P., Baldissera L., Alawi K. M., Keeble J., Nandi M., Brain S. D. (2017) Tail-Cuff Technique and Its Influence on Central Blood Pressure in the Mouse. J. Am. Heart Assoc. 6.

Yamashita K.-I., Uehara T., Prawiroharjo P., Yamashita K., Togao O., Hiwatashi A., Taniwaki Y., et al. (2019) Functional connectivity change between posterior cingulate cortex and ventral attention network relates to the impairment of orientation for time in Alzheimer's disease patients. Brain Imaging Behav. 13, 154-161.

Yanagisawa H., Davis E. C., Starcher B. C., Ouchi T., Yanagisawa M., Richardson J. A., Olson E. N. (2002) Fibulin-5 is an elastin-binding protein essential for elastic fibre development in vivo. Nature 415, 168-171.

Zhai Y., Yamashita T., Nakano Y., Sun Z., Shang J., Feng T., Morihara R., et al. (2016) Chronic Cerebral Hypoperfusion Accelerates Alzheimer's Disease Pathology with Cerebrovascular Remodeling in a Novel Mouse Model. J. Alzheimers. Dis. 53, 893-905. 


\section{Chapter 7}

\section{Discussion}




\section{Cerebral Amyloid Angiopathy and Cerebrovascular Hypoperfusion}

The decline of cerebral blood flow is known to occur during normal aging (Chen et al. 2011). However, the age-related mechanisms that account for, or are a result of, reduced cerebral blood flow during age is unknown. Observational studies in healthy adults have demonstrated that reduced cerebral blood flow during aging is independent of region-specific atrophy of the gray matter (Chen et al. 2011). Similarly, capillary density decreases with age in humans, non-human primates, and rodents (Amano et al. 1982; Bell and Ball 1990; Goldman et al. 1987; Jucker et al. 1990; Melamed et al. 1980) which may indicate an age-associated decline in density of the capillary network. It is possible that a threshold exists, separating the age-associated decline in capillary density and cerebral blood flow during normal aging from disease.

Cerebrovascular hypoperfusion is frequently observed in patients with $\mathrm{AD}$ (Johnson et al. 2005; Chen et al. 2011). The onset of cerebral hypoperfusion in the etiology of AD occurs early, before the development of A $\beta$ plaques, NFT tangles, and cerebral hypometabolism (Thomas et al. 2015; Zhang et al. 2018). The manifestation of hypoperfusion during the early stages of AD development supports its use as a clinical biomarker for the disease (Alsop et al. 2010; Chao et al. 2010). As in healthy aging, the mechanisms that underlie the deficits in cerebrovascular perfusion in $\mathrm{AD}$ remain unclear. Studies on transgenic mice that overexpress the APP gene have demonstrated a reduction in cerebral blood flow as part of the pathological events of AD progression (Weidensteiner et al. 2009; Faure et al. 2011; Poisnel et al. 2012; Hébert et al. 2013; Massaad et al. 2010). A critical question to the field that remains unanswered is why these transgenic mice that overexpress APP, demonstrate reduced cerebrovascular perfusion. The APP protein is a precursor to $A \beta_{1-42}$ and $A \beta_{1-40}$ production, both 
of which are found deposited in the parenchyma and on the walls of vessels in these transgenic mice. Although histopathological analysis of the brain in mice have demonstrated a reduced density of capillaries in regions around $\mathrm{A} \beta$ plaques (Kouznetsova et al. 2006), the deficits of cerebrovascular perfusion in these mice did not correlate to the density of the plaques (Weidensteiner et al. 2009).

\section{Cerebral Hypometabolism in Alzheimer's Disease}

Reduced cerebral metabolic demand may be another potential cause of the cerebrovascular hypoperfusion in APP mice. However, when cerebral glucose uptake is measured with $\left[{ }^{18} \mathrm{~F}\right]$-fluoro-2-deoxy-D-glucose PET, no correlation was found between metabolic deficit and the perfusion of cerebral tissue (Poisnel et al. 2012). In APP/PS1 transgenic mice at 3-months of age, cerebral glucose uptake is decreased in the hippocampus, cortex, and striatum; however, by 12-months of age, glucose uptake was increased in brain regions with persistent deficits in cerebrovascular perfusion. Impaired vascular function including vascular signaling (Niwa et al. 2001; Niwa et al. 2000), renin-angiotensin mechanism (Takeda et al. 2009), vascular tone, and blood perfusion, occur in APP mice prior to the onset of A $\beta$ plaque deposition (Han et al. 2008; Park et al. 2013; Faure et al. 2011; Hébert et al. 2013).

It is currently unknown whether CAA aggravates the functional deficits, or is a prerequisite to the dysfunction, of the vasculature. However, it is known that $A \beta$ can act directly on cerebral blood vessels resulting in dysfunction. This suggests that low levels of A $\beta$, before its accumulation on vessel walls and the development of CAA can induce vascular dysfunction and contribute to the development of cerebrovascular hypoperfusion in humans 
and rodents with $\mathrm{AD}$. The age at onset and the rate of $\mathrm{A} \beta$ deposition on the cerebral vasculature in mice is dependent on the specific mutation harbored by the APP gene. An earlier onset of CAA is observed in mice that harbor more than one autosomal dominant mutation as do Thy1APP751, TgCRND8, and Tg-SwDI transgenic mice. The development of CAA at later ages is seen in mice carrying a single familial mutation of the APP gene such as in Tg2576, APPDutch, APP23, APP/ London, PDAPP, and TgAPPArc transgenic mice. The age at onset of CAA can be shifted earlier if the mice carry a mutated presenilin gene in addition to an APP mutation as seen in APPswe/PS1dE9 and Thy1-APP751SLxHMG-PS1M146L transgenic mice. Empirical evidence has indicated that the ratio between $A \beta_{1-40}$ and $A \beta_{1-42}$ production is influential to the severity of AD and the age at onset of CAA (Herzig et al. 2004; Herzig et al. 2006; Herzig et al. 2007). In Tg-SwDI transgenic mice that carry the Swedish, Dutch, and Iowa APP mutation develop severe CAA with an onset at 6-months of age (Davis et al. 2004; Miao et al. 2005; Xu et al. 2007). Similarly, in Thy1-APP751SLxHMG-PS1M146L mice that carry the Swedish and London APP mutation develop severe CAA with an onset at 3-months of age (Blanchard et al. 2003; Schmitz et al. 2004; El Tayara et al. 2007; El Tannir El Tayara et al. 2010). In the 3xTg AD mouse line, which carry the PS1 M146V presenilin mutation, the Swedish hAPP695 APP mutation, in addition to K679M, N671L + htau (P301L) mutated tau develop A $\beta$ plaques at 6-months of age, however the onset of CAA remains to be determined (Oddo et al. 2003b; Oddo et al. 2003a; Billings et al. 2005; Fuhrmann et al. 2010). Hence, our first goal was to establish the severity and age at onset of cerebrovascular $A \beta$ accumulation in the $3 \times \operatorname{Tg} A D$ mouse model of $\mathrm{AD}$. To label $\mathrm{A} \beta$ in the brain of $3 \mathrm{xTg} A D$ mice we used anti- $\mathrm{A} \beta_{1-42}$ antibodies, 

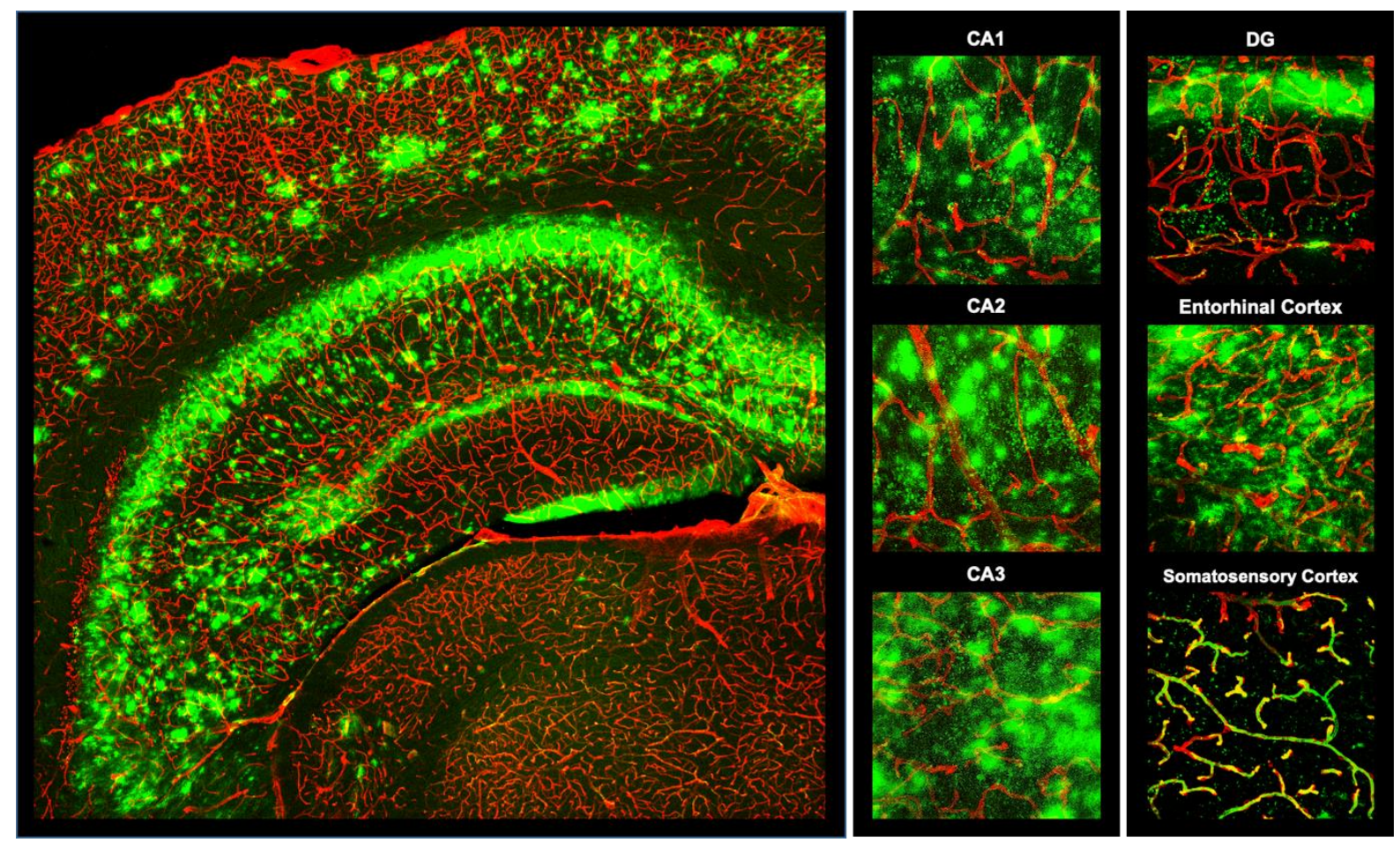

Figure 7.1. Image demonstrating the distribution and pattern of $A \beta$ deposition in the hippocampus and cortex. Confocal micrographs depicting the maximum intensity projection of a z-stack containing immunofluorescent labeling of collagen IV (red) and amyloid- $\beta$ 1-42 (green) in tissue sections of the brain from 24-month old 3xTg AD mice. Micrographs acquired at high magnification depict dense A $\beta$-plaque deposition in hippocampal subregions and vascular deposition in the cortex. 
methoxy-X04, and Congo red histological procedures for both the in vivo and ex vivo detection of $A \beta$ deposits in the parenchyma and cerebrovascular walls.

We found that the brain of $3 \times \mathrm{Tg}$ AD mice were severely affected by amyloidosis, having substantial deposition of $A \beta$ in both the parenchyma and around cerebral blood vessels at many of the critical brain regions that are implicated in the disease (Figure 7.1). These affected regions demonstrated much of the deposition of $A \beta$ on cerebral blood vessels occurs between the endothelium and the lamina densa of the basal lamina (Figure 7.2). This unique pattern of deposition indicates a compromised trans-endothelial cell transport system, a process that is essential for the clearance of $A \beta$ through the $\mathrm{BBB}$ and out of the brain.

It seems that during the early stages of $\mathrm{AD}$, prior to the deposition of $\mathrm{A} \beta$-plaque, tangles, hypometabolism, and hypoperfusion, the increased production of $A \beta$ exerts a state of chronic stress to the vascular endothelium. As age progresses and stress persists, the vascular endothelium cannot sustain their physiological functions within normal parameters, which initiates a cascade of pathological events including, reduced $A \beta$ clearance, which exacerbates endothelial dysfunction, ultimately resulting in vascular dysfunction and the degeneration of cerebral tissue of affected regions and the major pathological features of AD.

\section{Cerebrovascular Hypoperfusion, Mitochondrial Deficits, and Inflammation}

Astrocytes and microglia are central players involved in the development of inflammation of the central nervous system and both cells have been demonstrated to be associated with the localized inflammation of vascular $\mathrm{A} \beta$ deposits in $\mathrm{AD}$ (Grabowski et al. 2001; Eng et al. 2004; Zabel et al. 2013). Therefore, an aim of this project was to explore the metabolic changes in astrocytes that are associated with hypoxia as a model of hypoperfusion 

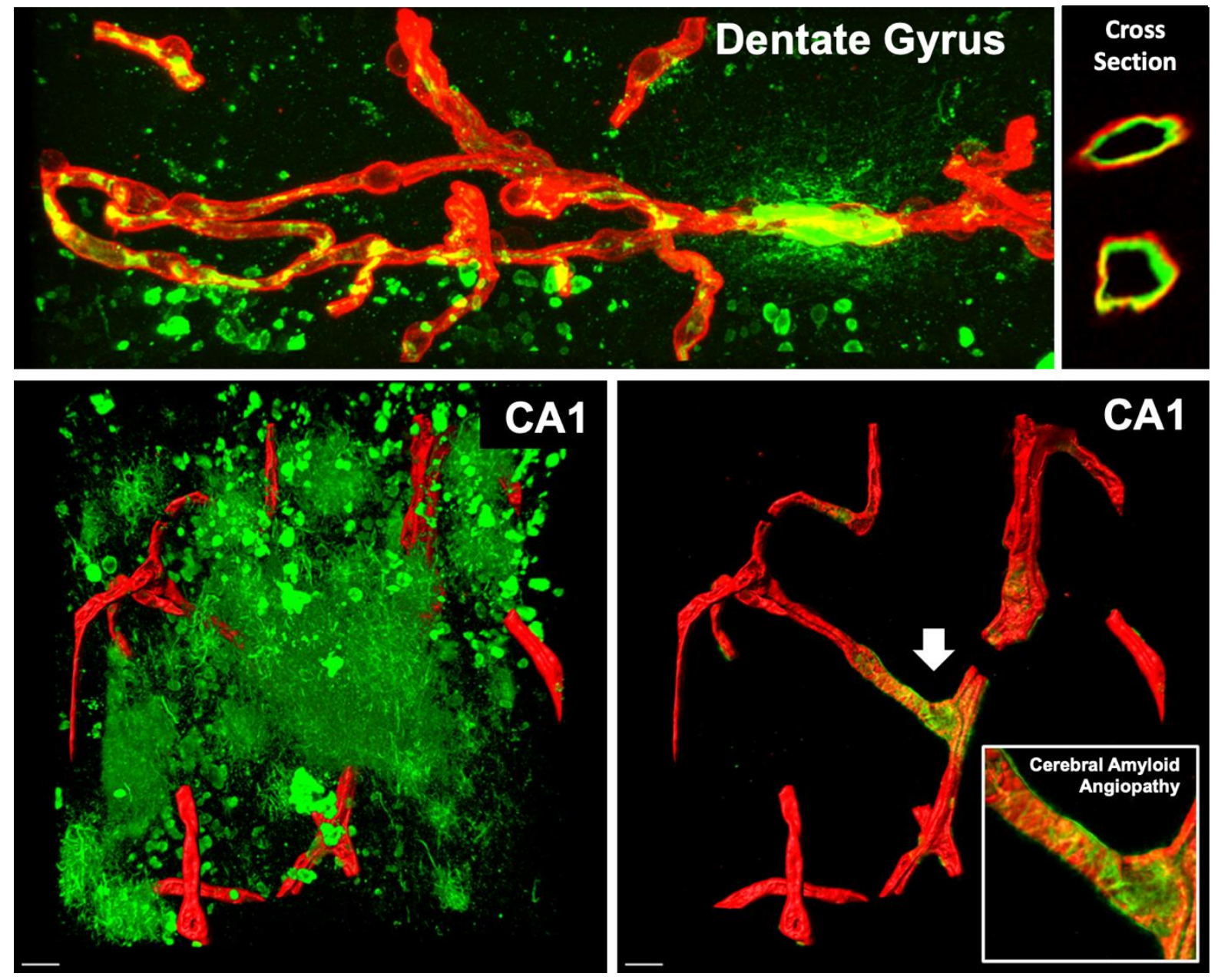

Figure 7.2. Image demonstrating the accumulation of amyloid- $\beta$ on the wall of cerebral blood vessels. Confocal micrographs at 63x magnification depicting immunofluorescence labeling of collagen IV (red) and amyloid- $\beta$ 1-42 (green) in the dentate gyrus and CA1 regions of the hippocampus in tissue sections of the brain from 24-month old 3xTg AD mice. Boxed region depicts a magnified area of a blood vessel with cerebral amyloid angiopathy. 
in vitro. The results obtained from this study suggest that hypoxia induces metabolic stressrelated changes to the mitochondrial network. In astrocytes exposed to 3-hours of hypoxia, the size distribution of the mitochondrial network shifted in favor of smaller mitochondria. Hypoxia resulted in the excessive fission of astrocytic mitochondria producing a numerous population of small mitochondria that resembled punctate. Mitochondrial fission was caused by the dephosphorylation of serine at residue 637 of the Drp-1 mitochondrial membrane protein. When astrocytes were reoxygenated for 10-hours, we observed a modest recovery to the original size distribution of mitochondria and the complete re-phosphorylation of the Drp1 protein. During hypoxia and following reoxygenation caused mitophagy of the hypoxiainduced population of small mitochondria, which resulted in a deficit of the total number of mitochondria and to the total mitochondrial area within astrocytes. These observations suggest that hypoperfusion in $\mathrm{AD}$ can cause mitochondrial deficits via upregulated mitophagy in astrocytes but also in cerebrovascular endothelial cells. Perhaps, the mitochondrial deficit we observed in astrocytes in response to hypoxia may occur from the hypoperfusion of tissue, and provide a reason for the development of hypometabolism in $\mathrm{AD}$ patients and in animal models. In astrocytes, we observed as many as $40 \%$ of all mitochondria being targeted for mitophagy following post-hypoxia reoxygenation. Indeed, previous studies have demonstrated mitochondrial number and functional deficits in human and animal models of AD with evidence of cerebrovascular hypoperfusion (Aliev et al. 2004; Moreira et al. 2010; Aliev et al. 2003a; Aliev et al. 2003b). We found that the intracellular ATP content after exposing primary astrocyte cultures to hypoxia for 3-hours caused a $64 \%$ decline in available ATP, suggesting that cellular ATP levels are likely depleted in hypoperfused mice in AD. During a metabolic crisis such as in AD, reduced intracellular ATP content may be insufficient to maintain normal 
cellular functions including $A \beta$ clearance by vascular endothelial cells. As stated above, we observed vascular deposition of $A \beta$ between the endothelium and lamina densa from fluorescently labeled tissue of 3xTg AD mice (Figure 7.2), which provides evidence of a faulty transendothelial clearance mechanism for $A \beta$ potentially explained by the acute sensitivity of mitochondria to hypoxic conditions, such as during cerebrovascular hypoperfusion.

\section{Cerebrovascular Hypoperfusion and Ischemic Injury in Alzheimer's}

\section{Disease}

The hallmark pathology of $\mathrm{AD}$ is the appearance of $\mathrm{A} \beta$ plaques and NFT in the brain, which leads to neuronal degeneration and cortical thinning (Walsh and Selkoe 2007). Additionally, it is well known that AD patients display white matter changes that are associated with the pathophysiology of AD (Barber et al. 1999; Englund 1998; Kim et al. 2015). The study presented in chapter 3 was aimed to determine if cerebrovascular hypoperfusion alone can produce parenchymal damage that is similar to the histopathology seen in AD. To address this aim, we developed a model to gradually induce cerebral hypoperfusion by bilateral carotid artery stenosis in mice using microcoils and ameroid constrictor rings. This procedure resulted in a gradual hypoperfusion that reduced cerebral blood flow to $43 \%$ of baseline over the course of 34 days. Histopathological analyses of the brain revealed neuronal and axonal damage throughout the brain, where the hippocampus was the most severely affected structure. We found evidence of gray matter injury that included; pyramidal cell loss in the CA1, CA2, and CA3 of the hippocampus; granular cell loss in the dentate gyrus; and parenchymal lesions in the globus pallidus internus and globus pallidus externus. In addition, we found white matter injury that was characterized by axonal disorganization and degeneration that most extensively 
affected the hippocampus and corpus callosum. The gradual development of hypoperfusion in these mice produced degenerative parenchymal injury that is similar to the neuronal degeneration in $\mathrm{AD}$. The progression of neuropathological changes in $\mathrm{AD}$ begin in the entorhinal cortex and the hippocampal formation, then spread to the temporal, parietal, and finally to the frontal association cortices (Braak and Braak 1996; Braak and Braak 1991; Hyman et al. 1984). Neuronal degeneration in AD is most severe in regions involved in memory function, including the entorhinal cortex and hippocampus and in cortical regions involved in language and executive function (Verma and Howard 2012; Lehmann et al. 2013). Cortical and hippocampal atrophy is seen in patients with AD (Sabuncu et al. 2011). Hippocampal atrophy is a marker of $\mathrm{AD}$ and the rate to which it occurs correlates with the change in clinical status of AD (Jack et al. 2000; Sabuncu et al. 2011). During healthy aging, $0.2-0.4 \%$ of the brain volume is lost per year, in aging patients with $\mathrm{AD}$, up to $10 \%$ of the brain volume is lost per year, particularly in regions that are vulnerable, such as the hippocampus (Fox et al. 1996; Fox et al. 2000; Andrews et al. 2013). Indeed, we found hippocampal and cortical atrophy in the brain of hypoperfused mice. The atrophic changes to the hippocampus included, pyramidal cell loss in the CA1, CA2, and CA3; granular cell loss in the DG; and diffuse axonal degeneration throughout the hippocampus. Similarly, loss of pyramidal cells in the neocortex and hippocampus is seen in $\mathrm{AD}$ and is a fundamental neuropathology of the disease (Mann 1996). The loss of hippocampal pyramidal cells in AD seems to predominantly occur in regions of the hippocampal formation (Davies et al. 1992). In a study the quantified the density of pyramidal cells in the postmortem brain of histopathologically confirmed AD patients found that the density of these cells decreased $44 \%$ in the subiculum, $28 \%$ in the prosubiculum, and $41 \%$ in the CA1 region of the hippocampus 
(Davies et al. 1992). Additionally, plaque deposition and NFT are more densely populated in regions affected with pyramidal cell loss (Davies et al. 1988). Hippocampal pyramidal cells are essential for the normal function of the hippocampus. Expectedly, the extent of pyramidal cell loss correlates with the clinical severity of dementia (Jack et al. 2000). A study of 144 patients that met the diagnostic criteria for $\mathrm{AD}$ that underwent magnetic resonance imaging (MRI) demonstrated that reduced hippocampal volume in patients with $\mathrm{AD}$ was more severe in patients with one or more vascular risk factors (Dhikav and Anand 2011), linking the vascular factors to the development of hippocampal atrophy in AD (Dhikav and Anand 2012).

We found white matter damage in hypoperfused mice that consisted of diffused axonal injury, axonal disorganization, axonal degeneration, and vacuolation. Additionally, we observed corpus callosal thinning and degeneration to the fornix in the brain of hypoperfused mice. Parenchymal vacuolation, also called spongiform degeneration, typically occurs in white matter regions of the brain where dendrites and myelinated axons degenerate. Cerebrovascular injury and microcirculation dysfunction can result in oligodendrocyte apoptosis and the vacuolation of the parenchyma in heroin addicts (Yin et al. 2013). Vacuolation in AD is found in the medial temporal lobe, amygdala, and upper layers of the isocortex and less common in the limbic lobe (Smith et al. 1987; Mancardi et al. 1982). In a study using spin-echo magnetic resonance imaging and proton magnetic spectroscopic imaging revealed axonal injury and membrane alterations in AD patients (Meyerhoff et al. 1994). Axonal transport may fail during the progression of AD (Cash et al. 2003). Senile plaques are linked to alterations of the axonal compartment (Terry 1996), as they are composed of dystrophic neurites that correspond to axonal swellings containing aberrant accumulations of axonal cargo and tau (Masliah et al. 1993). These observations indicate that the prominent white matter injury in AD patients, that 
is characterized by axonal injury, vacuolation, axonal transport failure, and dystrophic neurites can be caused by vascular dysfunction alone. This is further supported by a study of AD patients with cerebrovascular disease, which demonstrated extensive swelling and vacuolation of white matter astrocytes that contained beaded and/or disintegrated processes (Tomimoto $e t$ al. 1997). These astrocytes were immunoreactive for serum proteins, which are known to induce astrocyte activation (Tomimoto et al. 1997). This suggests that vascular dysfunction and impaired BBB is sufficient to induce the activation of astrocytes and white matter damage. This is in agreement with our study reported in chapter 2, demonstrating that the exposure of primary astrocytes to hypoxia can cause metabolic changes and retraction of astrocytic extensions, indicative of cellular reactivity and the activation of astrocytes.

Astrocytes perform functions that are essential to the homeostasis and ongoing function of the central nervous system. One of these important functions is the regulation of immune response by modulating inflammation via inflammatory signaling. In $\mathrm{AD}$, astrocytes express apolipoprotein E and participate in the degradation and removal of $\mathrm{A} \beta$ (González-Reyes et al. 2017). Excessive cerebral inflammation and/or reactive oxygen species can affect astrocytic process that involve calcium signaling regulation, NADPH oxidase, glutamate uptake, and mitochondrial function (González-Reyes et al. 2017). These cellular changes can induce damage to the parenchyma including, oxidation of proteins and lipids, calcium dysregulation, and excitotoxicity (González-Reyes et al. 2017). We found polynuclear lesions in white matter regions of hypoperfused mice. These lesions are likely a result of activated astrocytes that result from the hypoxic environment, serum proteins, and damage associated molecular patterns that were caused by hypoperfusion-related injury. In $\mathrm{AD}$, astrocytes are a major area of research, as they are commonly associated with the neuronal damage seen in AD. It is likely 
that early cerebrovascular dysfunction results in astrocyte activation leading to the initial neuronal damage in AD. Furthermore, activation of astrocytes by $\mathrm{A} \beta$ can be potentiated by oxidants (García-Matas et al. 2010), such as hypoxia during hypoperfusion. Since nonactivated astrocytic function is reduced when activation occurs, astrocytic degradation of $\mathrm{A} \beta$ and its removal should also be reduced. These changes to the cellular environment would exacerbate parenchymal damage, including enhanced vascular injury. In addition to the increased oxidative stress and inflammatory signaling, vascular endothelial cells would be required to clear parenchymal $A \beta$ without astrocytic degradation of the polymers, a potential initiating factor leading to the development of CAA.

\section{Amyloid-ß-mediates Mitochondrial Dysfunction and Cell Death in Vascular}

\section{Endothelial Cells}

The mitochondrial cascade hypothesis attempts to explain late-onset $\mathrm{AD}$, where the dysfunction of mitochondria initiates the deposition of $A \beta$, NFT formation, and synaptic degeneration (Swerdlow et al. 2014; Swerdlow and Khan 2009). Mitochondrial dysfunction and oxidative stress are important factors in the early pathogenesis of AD (Moreira et al. 2006; Moreira et al. 2009; Su et al. 2008; Nunomura et al. 2001). A $\beta$ plaques are found clustered around regions with damaged and degenerated mitochondria (Xie et al. 2013). Dysfunctional

mitochondrial enzymes including, cytochrome c oxidase (COX), $\alpha$-ketoglutarate dehydrogenase complex, and pyruvate dehydrogenase complex also contribute to the pathogenesis of AD (Silva et al. 2012; Maurer et al. 2000; Parker et al. 1990). Reduced COX activity has been documented in hippocampal neurons (Cottrell et al. 2002; Maurer et al. 2000) and in cortical neurons (Mutisya et al. 1994; Maurer et al. 2000) of AD patients. 
Previously, the specific intracellular events induced by A $\beta$ that mediate the changes to mitochondria in $\mathrm{AD}$ is largely unknown. The goal of the study described in chapter 4 aimed to determine the mechanism in which $\mathrm{A} \beta$ mediates the dysfunction of mitochondria in $\mathrm{AD}$. To address this aim, we studied the effects of $\mathrm{A} \beta$ on the bEnd.3 cell line and primary cerebrovascular endothelial cell cultures. We found that the exposure of cerebrovascular endothelial cells to $\mathrm{A} \beta$ accelerated mitochondrial oxygen consumption and increased superoxide production. We found that these changes occurred via accumulated calcium in the mitochondrial matrix and could be prevented by chelating extracellular calcium. Although dysregulated calcium homeostasis is well documented in AD research (Jadiya et al. 2019; LaFerla 2002), its roll in cerebrovascular dysfunction in AD is limited. Calcium signaling mediates vascular endothelial cell sensing of shear stress via P2X4 receptor (Yamamoto et al. 2000; Kwan et al. 2003), an important mechanism that allows the vascular endothelium to adapt to dynamic changes of blood flow. Disrupted endothelial cell calcium signaling is likely a factor involved in the pathological events leading to the development of tortuous vessels. Additional calcium entering the cell by mitochondria-produced superoxide anions via redoxsensitive NSCC can increase mitochondrial calcium accumulation, progressing the respiratory dysfunction. Although, metabolic function is reduced in patients at late stages of $\mathrm{AD}$, studies have documented a hypermetabolic phenotype in rodent models at earlier stages (Knight et al. 2012; Snellman et al. 2019; Bouter and Bouter 2019; Luo et al. 2012). This early elevation of mitochondrial respiration may mediate the initial mitochondrial damage and endothelial cell stress that results in dysfunction of critical cellular process and contribute to $\mathrm{A} \beta$ accumulation as the disease progresses. 
Deficits in function and the transport of mitochondria have been reported in primary cultures exposed to A $\beta$ (Calkins and Reddy 2011; Rui and Zheng 2016; Rui et al. 2006) and from 3xTg AD cultured neurons overexpressing mutated APP (Cavendish et al. 2019). Additionally, mitochondrial morphology is altered AD (Hirai et al. 2001b; Moreira et al. 2007). When we exposed vascular endothelial cells to $A \beta$ we found that mitochondrial size reduced via a process that was consistent with stress-induced fission. This is consistent with the literature that documents excessive mitochondrial fission in the brain of both patients and animal models of AD (Manczak et al. 2011; Kandimalla and Reddy 2016; Hirai et al. 2001a; Lu et al. 2018). This provides a potential mechanism for the observation of increased mitochondrial fission in $\mathrm{AD}$, where $\mathrm{A} \beta$-induced stress causes mitochondrial fission in vascular endothelial cells and likely in other cell types. Additionally, the $A \beta$-induced mitochondrial fission could be prevented when we chelated extracellular calcium, indicating a prominent role in the stress-related signaling that results from $A \beta$ exposure.

Ultimately, endothelial cell exposure to $A \beta$ resulted in cell death, possibly via a mitochondrial mediated mechanism.

\section{Cerebrovascular Corrosion Casting and MicroCT Imaging for the Assessment of the Cerebrovasculature}

Imaging of the mammalian brain and its systems has long been a goal of neuroscience research. However, acquiring images of structures deep within the brain has proven difficult to obtain without the need to slice the tissue into thin sections. This difficulty is caused by interference from the tissue, which results in reduced efficiency and resolution of the collected images. This is a serious limitation; poor-quality images produce inaccurate interpretation of 
the data and potentially mislead the scientific community. The development of more advanced imaging methods for deep-brain structures is crucial for continuing progress of the field. This is especially true for highly complex systems such as the cerebrovascular network. The unique nature of the blood vessel geometry and the complex network(s) that they comprise are highly dimensional, as such, image data collection of vascular networks from sections of sliced tissue is impractical for analyses of vascular network composition and organization.

Therefore, the study described in chapter 5 aimed to develop the methodology required to study the complete cerebrovascular network from the entire brain. A secondary goal of this study was to develop an array of analyses that could be used to evaluate the angioarchitecture in a biologically meaningful manner. We focused on developing a procedure that enables the collection of volumetric imaging data at micron-level resolution of the complete cerebrovascular network of the whole brain of adult female and male mice. This is a crucial advance, as many contemporary imaging studies of the cerebrovasculature have been limited to cubic millimeters of brain. Additionally, literature on sex differences of the cerebrovasculature beyond vessel counting is limited. In complex systems such as vascular networks, major differences can be found beyond the absolute number of vessels, such as the particular topology of vascular networks, which can influence the efficiency, stability, or redundancy of blood flow to the brain and its subregions.

We reported for the first time, three-dimensional data sets of the cerebrovascular network over the entire brain from nine mice and evaluate the angioarchitecture at the macroand microscopic level in female and male mice. We provided a characterization of the cerebrovasculature by measuring the geometry of the angioarchitecture. We then mapped the topology of the vascular network to generate morphometric descriptions of deconstructed 
network components, assessed the connectivity, and provided global metrics of the network angioarchitecture. These analyses revealed sexual dimorphic traits of the organization of the vascular network, where female mice demonstrated more complex and thorough network structures. Additionally, female mice contained on average a greater density of microvessels demonstrating smaller intervessel distances than did male mice. Overall, we performed over 30 separate analyses to characterize the cerebrovasculature in female and male mice and revealed a number of parameters that qualified as sexually dimorphic. This work may prove crucial to the field by providing a new perspective to explain the reasons why sensitivity to some diseases are different in females and males.

A thorough assessment of the cerebrovasculature in $\mathrm{AD}$ has not been previously reported. This shortcoming is broadly due to the difficulty of acquiring volumetric datasets of cerebrovascular networks that are sufficiently robust for accurate interpretations of the dimensional properties of network structures. This is important for AD research, in that, due to the dynamic nature of the cerebrovasculature, chronic neurodegenerative diseases that progress with age are likely to cause structural changes to the vascular network, which may participate in the pathophysiology of disease. For example, cerebrovascular malformations that can develop in response to hemodynamic stress over long periods of time. Vascular tortuosity is a prominent cerebrovascular malformation found in the brain of patients and rodents with AD (Dorr, J. Brain). Elevated blood flow can injure the wall of blood vessels, causing the aberrant deposition of basement membrane components, the degradation of elastin, and the elongation of blood vessels. These pathogenic changes to the vessel wall can cause the blood vessel to become dysmorphic and often results in a vessel becoming tortuous (Dobrin et al. 1988; Carta et al. 2009; Taarnhøj et al. 2008; Coucke et al. 2006). 
In $\mathrm{AD}$, a major source of damage to the cerebrovasculature is caused by $\mathrm{A} \beta$ (Selkoe and Hardy 2016). Particularly, cerebral capillaries are responsible for $85 \%$ of the clearance of A $\beta$ from the brain (Deane et al. 2008; Deane et al. 2004; Shibata et al. 2000; Zlokovic 2008). It is conceivable that age-associated capillary dysfunction compromises the efficiency of A $\beta$ clearance through capillary endothelial cells, initiating the onset of CAA and subsequently, the pathogenic cascade responsible for the manifestation of $\mathrm{AD}$. The particular vascular risk factor(s) or combination, harbored by an individual may be decisive to whether capillary function is compromised during aging. Cerebrovascular dysfunction that weakens transendothelial transport and/or lysosomal degradation would result in the progressive accumulation of $A \beta$, enhancing endothelial cell stress and promoting apoptosis. Early endothelial cell death can cause vessel regression and the thinning of capillary network density. Early loss of capillary network density and concomitant dysfunction of transendothelial cell transport may mark the onset of A $\beta$-plaque development and CAA. A goal for the study described in chapter 5 was to develop analytical solutions to characterize the magnitude, complexity, and organization of the angioarchitecture in the brain. These assessments would allow us to determine if early changes to the cerebrovasculature occur in $\mathrm{AD}$ and to characterize the progression of these changes with age.

\section{Changes to the Cerebrovasculature in AD Occurs Early and Progresses with}

\section{Age}

In his seminal work, Alzheimer documented observations of vascular pathologies and their comorbidities in his original report (Alzheimer 1907). Since then, a growing body of research has established an association with the occurrence of $\mathrm{AD}$ and vascular factors (Montagne et al. 2016; Dickstein et al. 2010; Murray et al. 2011; Shin et al. 2007; Grammas 
et al. 2002). Although, reports documenting changes to the cerebrovasculature in $\mathrm{AD}$ have overwhelmingly indicated that vascular network density decreases with AD (Sagare et al. 2013; Vinters et al. 1994; Kalaria and Hedera 1995; Miyakawa et al. 1988; Hashimura et al. 1991), several studies have demonstrated the opposite observation, where vascular density is increased (Desai et al. 2009; Durrant et al. 2020; Ek Olofsson and Englund 2019). This is further confounded by conflicting studies that demonstrate both hypermetabolism and hypometabolism in mouse models of AD (Knight et al. 2012; Snellman et al. 2019; Bouter and Bouter 2019; Luo et al. 2012). However, past studies of the cerebrovasculature in humans and in rodents with $\mathrm{AD}$ have largely been limited to tissue sections and small volumetric datasets restricted to selected brain regions. Although these past studies have provided important information, there exists a need to describe the vascular changes over the entire brain while also providing information regarding the connective organization of the cerebrovasculature, which is more closely tied to its function. Therefore, the study described in chapter 6 was aimed to characterize the changes to the cerebrovasculature at early stages of $\mathrm{AD}$ and how these changes progress with age. Reconstruction of the cerebrovasculature in three-dimensions revealed evidence of chronic vascular injury in $3 \times \mathrm{Tg} \mathrm{AD}$ mice including, tortuous vessels in the cortex and aneurysms of capillaries and arterioles. Morphometric analysis of the angioarchitecture revealed early changes to the cerebrovasculature that indicated a structural response to chronic injury. Interestingly, these early changes were observed at 3-months of age, and included alterations to the average vessel diameter, intervessel distance, and the redundancy of pathways, suggesting altered network composition. Consistent with the literature, analysis of the whole-brain that measured the volume of vessels as a function of average vessel diameter indicated that the majority of these early changes were accounted for 
by changes to the microvasculature. Although, reports documenting changes to the cerebrovasculature in $\mathrm{AD}$ have overwhelmingly indicated that vascular network density decreases with $\mathrm{AD}$, several studies have demonstrated the opposite observation, where vascular density is increased. Indeed, at 3-months, 3xTg AD mice demonstrated an increased amount of microvessels compared to age-matched WT mice. This elevated microvascular content in $3 \times \mathrm{Tg} \mathrm{AD}$ mice persisted to 6-months of age. Increased microvascular volume in $3 \times \operatorname{Tg} \mathrm{AD}$ mice may be associated with the proangiogenic properties of $\mathrm{A} \beta$ (Biron et al. 2011; Zand et al. 2005; Fioravanzo et al. 2010). Additionally, endothelial cell stress can stimulate cell proliferation and the growth of new vessels. Early chronic damage to the microvasculature can cause reoccurring regression and growth of microvessels that may drastically change the connectivity of the network and affect the pathways and efficiency of blood flow through the vascular network.

The changes at 3-and 6-months were in contrast to the changes to the angioarchitecture at later ages. Whole brain analysis indicated a decline in the number of vessel segments that occurred along with reduced vascular volume, vessel surface area, and a greater intervessel distance in 3xTg AD mice at late ages. Late age in 3xTg AD mice was also associated with reduced number of vessel-to-vessel junctions and compromised network complexity in $3 \times \mathrm{Tg}$ $\mathrm{AD}$ mice. These late changes are consistent with the majority of the literature that suggest a reduced capillary density in $\mathrm{AD}$.

Declarative memory is the first cognitive domain affected during the progression of AD. The hippocampus and the medial temporal lobe are critical regions for declarative memory function (Stoub et al. 2006; Zola-Morgan et al. 1986). When we evaluated the cerebrovasculature of the hippocampal formation, we found early differences to the number of 
vessel segments and vessel-to-vessel junctions at 3-months of age. These early changes most severely affected the CA1, DG, CA3, and then ENT CTX, in that order. Similar to our wholebrain analysis, the hippocampus demonstrated microvascular degeneration that began at 6months and progressed with age.

Sensory input form the primary somatosensory cortex and association cortex project information to the hippocampus through the perforant path; the loss of layer II neurons in the entorhinal cortex can cause a disconnection of information transmitted to the hippocampus (Hyman et al. 1984; Amaral et al. 1987; Van Hoesen et al. 1975). We found the angioarchitecture of the MO PFC to be the most severely affected cortical region. At 3-months, the vasculature of the CC and not the MO PFC and SS CTX, demonstrated a reduced number of vessel segments and a lower count of vessel-to-vessel junctions. At 6-months of age, the MO PFC contained a reduced number of segments, vessel junctions, total vessel volume, surface area, and intervessel distance. Whereas, the angioarchitecture of the SS CTX and CC provided a reduced surface area of vessels and a greater distance between vessels relative to age-matched WT mice. Interestingly, at 12- and 24-months of age, an increased intervessel distance was the only measured parameter to be affected and in all cortical regions assessed.

Overall, our data demonstrate that the cerebrovasculature of 3xTg AD mice is affected early during aging and is characterized by morphological and structural adaptations that are likely caused by chronic injury to vessels. These data indicated that alteration to the angioarchitecture occurs before the onset of plaque development. Once plaque deposition occurs, changes to the angioarchitecture begin to indicate structural changes that occur via vascular degeneration. These data suggest that changes to the vasculature that occur early 
during $\mathrm{AD}$ may progress, resulting in cerebrovascular degeneration, initiating the onset of clinically detectable $\mathrm{AD}$ in patients.

\section{Summary of Dissertation Research}

We have demonstrated that pathological changes to the cerebrovasculature is a prominent event during $\mathrm{AD}$. We have demonstrated age-related changes to the angioarchitecture over the whole-brain in 3xTg $\mathrm{AD}$ mice that occurred early, before the onset of A $\beta$-plaque deposition and NFT development in 3xTg AD mice. We have provided evidence indicating that the microvasculature is dramatically affected over the entire brain and in key brain regions that are implicated in the behavioral and cognitive deficits in AD. Additionally, we have demonstrated that $A \beta$ is cytotoxic to vascular endothelial cell, causing dysregulated oxidative phosphorylation that resulted in aberrant ATP synthesis and enhanced superoxide production. We found that mitochondrial calcium is critically involved in the $A \beta$-induced changes to mitochondrial function and endothelial cell death. We have provided visual evidence depicting $A \beta$ deposition on cerebrovascular walls, including microvessels. The proximity of $A \beta$ to the endothelium of these vessels suggests nascent endothelial injury and apoptosis via the mechanisms described above. Vascular degeneration, especially microvascular degeneration can result in an insufficient perfusion of blood to the parenchyma. We have demonstrated in astrocytes, a major participant of CNS inflammatory regulation that reduced oxygen delivery can cause metabolic stress, characterized by excessive mitochondrial fission, mitophagy, and potentially astrocytic activation. These changes to astrocytes may prime the CNS for an inflammatory state. Furthermore, we have demonstrated that hypoperfusion alone, results in parenchymal injury that presents itself similarly to the neuronal degeneration in AD. Hypoperfusion caused both gray matter and white matter injury that 
primarily affected the hippocampus and cortex of mice. The damage to white matter included axonal dystrophy, disarrangement, and degeneration that is similar to the histological observations from AD tissue. Additionally, we observed vacuolation of the parenchyma in hypoperfused mice, which is known to form after the degeneration of dendrites and axons.

Overall, these findings provide a basis for the development of novel therapeutic strategies that target the changes to the cerebrovasculature for the treatment of AD. Our data revealed a critical period of cerebrovascular dysfunction and degeneration during the early presymptomatic stages of $\mathrm{AD}$. This observation may pave the way for the development of a prophylactic strategy for the treatment of $\mathrm{AD}$ by preventing the initial factors that lead to compromised cerebrovasculature.

\section{References}

Aliev G., Obrenovich M. E., Smith M. A., Perry G. (2003a) Hypoperfusion, Mitochondria Failure, Oxidative Stress, and Alzheimer Disease. J. Biomed. Biotechnol. 2003, $162-$ 163.

Aliev G., Smith M. A., la Torre J. C. de, Perry G. (2004) Mitochondria as a primary target for vascular hypoperfusion and oxidative stress in Alzheimer's disease. Mitochondrion 4, 649-63.

Aliev G., Smith M. A., Obrenovich M. E., La Torre J. C. De, Perry G. (2003b) Role of vascular hypoperfusion-induced oxidative stress and mitochondria failure in the pathogenesis of Alzheimer disease. Springer New York LLC.

Alsop D. C., Dai W., Grossman M., Detre J. A. (2010) Arterial spin labeling blood flow MRI: Its Role in the early characterization of Alzheimer's disease. IOS Press.

Alzheimer A. (1907) Ueber eine eigenartige erkrankung der hirnrinde. Z Gesamte. Neurol Psychiatr 18, 177-179. 
Amano T., Meyer J. S., Okabe T., Shaw T., Mortel K. F. (1982) Stable xenon ct cerebral blood flow measurements computed by a single compartment double integration model in normal aging and dementia. J. Comput. Assist. Tomogr. 6, 923-932.

Amaral D. G., Insausti R., Cowan W. M. (1987) The entorhinal cortex of the monkey: I. Cytoarchitectonic organization. J. Comp. Neurol. 264, 326-355.

Andrews K. A., Modat M., Macdonald K. E., Yeatman T., Cardoso M. J., Leung K. K., Barnes J., et al. (2013) Atrophy rates in asymptomatic amyloidosis: implications for Alzheimer prevention trials. PLoS One 8, e58816.

Barber R., Scheltens P., Gholkar A., Ballard C., McKeith I., Ince P., Perry R., O’Brien J. (1999) White matter lesions on magnetic resonance imaging in dementia with Lewy bodies, Alzheimer's disease, vascular dementia, and normal aging. J. Neurol. Neurosurg. Psychiatry 67, 66-72.

Bell M. A., Ball M. J. (1990) Neuritic plaques and vessels of visual cortex in aging and Alzheimer's dementia. Neurobiol. Aging 11, 359-370.

Billings L. M., Oddo S., Green K. N., McGaugh J. L., LaFerla F. M. (2005) Intraneuronal Abeta causes the onset of early Alzheimer's disease-related cognitive deficits in transgenic mice. Neuron 45, 675-688.

Biron K. E., Dickstein D. L., Gopaul R., Jefferies W. A. (2011) Amyloid Triggers Extensive Cerebral Angiogenesis Causing Blood Brain Barrier Permeability and Hypervascularity in Alzheimer's Disease. PLoS One 6, e23789.

Blanchard V., Moussaoui S., Czech C., Touchet N., Bonici B., Planche M., Canton T., et al. (2003) Time sequence of maturation of dystrophic neurites associated with $A \beta$ deposits in APP/PS1 transgenic mice. Exp. Neurol. 184, 247-263.

Bouter C., Bouter Y. (2019) (18)F-FDG-PET in Mouse Models of Alzheimer's Disease. Front. Med. 6, 71.

Braak H., Braak E. (1991) Neuropathological stageing of Alzheimer-related changes. Acta Neuropathol. 82, 239-259.

Braak H., Braak E. (1996) Development of Alzheimer-related neurofibrillary changes in the 
neocortex inversely recapitulates cortical myelogenesis. Acta Neuropathol. 92, 197201.

Calkins M. J., Reddy P. H. (2011) Amyloid beta impairs mitochondrial anterograde transport and degenerates synapses in Alzheimer's disease neurons. Biochim. Biophys. Acta 1812, 507-513.

Carta L., Wagenseil J. E., Knutsen R. H., Mariko B., Faury G., Davis E. C., Starcher B., Mecham R. P., Ramirez F. (2009) Discrete contributions of elastic fiber components to arterial development and mechanical compliance. Arterioscler. Thromb. Vasc. Biol. 29, 2083-2089.

Cash A. D., Aliev G., Siedlak S. L., Nunomura A., Fujioka H., Zhu X., Raina A. K., et al. (2003) Microtubule reduction in Alzheimer's disease and aging is independent of tau filament formation. Am. J. Pathol. 162, 1623-1627.

Cavendish J. Z., Sarkar S. N., Colantonio M. A., Quintana D. D., Ahmed N., White B. A., Engler-Chiurazzi E. B., Simpkins J. W. (2019) Mitochondrial Movement and Number Deficits in Embryonic Cortical Neurons from 3xTg-AD Mice. J. Alzheimers. Dis. 70, $139-151$.

Chao L. L., Buckley S. T., Kornak J., Schuff N., Madison C., Yaffe K., Miller B. L., Kramer J. H., Weiner M. W. (2010) ASL perfusion MRI predicts cognitive decline and conversion from MCI to dementia. Alzheimer Dis. Assoc. Disord. 24, 19-27.

Chen J. J., Rosas H. D., Salat D. H. (2011) Age-associated reductions in cerebral blood flow are independent from regional atrophy. Neuroimage 55, 468-478.

Cottrell D. A., Borthwick G. M., Johnson M. A., Ince P. G., Turnbull D. M. (2002) The role of cytochrome c oxidase deficient hippocampal neurones in Alzheimer's disease. Neuropathol. Appl. Neurobiol. 28, 390-396.

Coucke P. J., Willaert A., Wessels M. W., Callewaert B., Zoppi N., Backer J. De, Fox J. E., et al. (2006) Mutations in the facilitative glucose transporter GLUT10 alter angiogenesis and cause arterial tortuosity syndrome. Nat. Genet. 38, 452-457.

Davies D. C., Horwood N., Isaacs S. L., Mann D. M. (1992) The effect of age and Alzheimer's disease on pyramidal neuron density in the individual fields of the 
hippocampal formation. Acta Neuropathol. 83, 510-517.

Davies D. C., Wilmott A. C., Mann D. M. (1988) Senile plaques are concentrated in the subicular region of the hippocampal formation in Alzheimer's disease. Neurosci. Lett. 94, 228-233.

Davis J., Xu F., Deane R., Romanov G., Previti M. Lou, Zeigler K., Zlokovic B. V, Nostrand W. E. Van (2004) Early-onset and robust cerebral microvascular accumulation of amyloid beta-protein in transgenic mice expressing low levels of a vasculotropic Dutch/Iowa mutant form of amyloid beta-protein precursor. J. Biol. Chem. 279, 2029620306.

Deane R., Sagare A., Hamm K., Parisi M., Lane S., Finn M. B., Holtzman D. M., Zlokovic B. V (2008) apoE isoform-specific disruption of amyloid beta peptide clearance from mouse brain. J. Clin. Invest. 118, 4002-4013.

Deane R., Wu Z., Sagare A., Davis J., Yan S. Du, Hamm K., Xu F., et al. (2004) LRP/amyloid beta-peptide interaction mediates differential brain efflux of Abeta isoforms. Neuron 43, 333-344.

Desai B. S., Schneider J. A., Li J.-L., Carvey P. M., Hendey B. (2009) Evidence of angiogenic vessels in Alzheimer's disease. J. Neural Transm. 116, 587-597.

Dhikav V., Anand K. (2011) Potential predictors of hippocampal atrophy in Alzheimer's disease. Drugs Aging 28, 1-11.

Dhikav V., Anand K. S. (2012) Are vascular factors linked to the development of hippocampal atrophy in Alzheimer's disease? J. Alzheimers. Dis. 32, 711-718.

Dickstein D. L., Walsh J., Brautigam H., Stockton S. D. J., Gandy S., Hof P. R. (2010) Role of vascular risk factors and vascular dysfunction in Alzheimer's disease. Mt. Sinai J. Med. 77, 82-102.

Dobrin P. B., Schwarcz T. H., Baker W. H. (1988) Mechanisms of arterial and aneurysmal tortuosity. Surgery 104, 568-571.

Durrant C. S., Ruscher K., Sheppard O., Coleman M. P., Özen I. (2020) Beta secretase 1dependent amyloid precursor protein processing promotes excessive vascular sprouting 
through NOTCH3 signalling. Cell Death Dis. 11, 98.

Ek Olofsson H., Englund E. (2019) A cortical microvascular structure in vascular dementia, Alzheimer's disease, frontotemporal lobar degeneration and nondemented controls: a sign of angiogenesis due to brain ischaemia? Neuropathol. Appl. Neurobiol. 45, 557569.

Eng J. A., Frosch M. P., Choi K., Rebeck G. W., Greenberg S. M. (2004) Clinical manifestations of cerebral amyloid angiopathy-related inflammation. Ann. Neurol. 55, 250-256.

Englund E. (1998) Neuropathology of white matter changes in Alzheimer's disease and vascular dementia. Dement. Geriatr. Cogn. Disord. 9 Suppl 1, 6-12.

Faure A., Verret L., Bozon B., Tannir El Tayara N. El, Ly M., Kober F., Dhenain M., Rampon C., Delatour B. (2011) Impaired neurogenesis, neuronal loss, and brain functional deficits in the APPxPS1-Ki mouse model of Alzheimer's disease. Neurobiol. Aging 32, 407-418.

Fioravanzo L., Venturini M., D. Liddo R., Marchi F., Grandi C., P. Parnigotto P., Folin M. (2010) Involvement of Rat Hippocampal Astrocytes in b-Amyloid-Induced Angiogenesis and Neuroinflammation. Curr. Alzheimer Res. 7, 591-601.

Fox N. C., Cousens S., Scahill R., Harvey R. J., Rossor M. N. (2000) Using serial registered brain magnetic resonance imaging to measure disease progression in Alzheimer disease: power calculations and estimates of sample size to detect treatment effects. Arch. Neurol. 57, 339-344.

Fox N. C., Freeborough P. A., Rossor M. N. (1996) Visualisation and quantification of rates of atrophy in Alzheimer's disease. Lancet (London, England) 348, 94-97.

Fuhrmann M., Bittner T., Jung C. K. E., Burgold S., Page R. M., Mitteregger G., Haass C., Laferla F. M., Kretzschmar H., Herms J. (2010) Microglial Cx3cr1 knockout prevents neuron loss in a mouse model of Alzheimer's disease. Nat. Neurosci. 13, 411-413.

García-Matas S., Vera N. de, Aznar A. O., Marimon J. M., Adell A., Planas A. M., Cristòfol R., Sanfeliu C. (2010) In vitro and in vivo activation of astrocytes by amyloid-beta is potentiated by pro-oxidant agents. J. Alzheimers. Dis. 20, 229-245. 
Goldman H., Berman R. F., Gershon S., Murphy S. L., Altman H. J. (1987) Correlation of behavioral and cerebrovascular functions in the aging rat. Neurobiol. Aging 8, 409-416.

González-Reyes R. E., Nava-Mesa M. O., Vargas-Sánchez K., Ariza-Salamanca D., MoraMuñoz L. (2017) Involvement of Astrocytes in Alzheimer's Disease from a Neuroinflammatory and Oxidative Stress Perspective. Front. Mol. Neurosci. 10, 427.

Grabowski T. J., Cho H. S., Vonsattel J. P., Rebeck G. W., Greenberg S. M. (2001) Novel amyloid precursor protein mutation in an Iowa family with dementia and severe cerebral amyloid angiopathy. Ann. Neurol. 49, 697-705.

Grammas P., Yamada M., Zlokovic B. (2002) The cerebromicrovasculature: a key player in the pathogenesis of Alzheimer's disease. J. Alzheimers. Dis. 4, 217-223.

Han B. H., Zhou M. L., Abousaleh F., Brendza R. P., Dietrich H. H., Koenigsknecht-Talboo J., Cirrito J. R., Milner E., Holtzman D. M., Zipfel G. J. (2008) Cerebrovascular dysfunction in amyloid precursor protein transgenic mice: Contribution of soluble and insoluble amyloid- $\beta$ peptide, partial restoration via $\gamma$-secretase inhibition. J. Neurosci. 28, 13542-13550.

Hashimura T., Kimura T., Miyakawa T. (1991) Morphological changes of blood vessels in the brain with Alzheimer's disease. Jpn. J. Psychiatry Neurol. 45, 661-665.

Hébert F., Grand’Maison M., Ho M. K., Lerch J. P., Hamel E., Bedell B. J. (2013) Cortical atrophy and hypoperfusion in a transgenic mouse model of Alzheimer's disease. Neurobiol. Aging 34, 1644-1652.

Herzig M. C., Nostrand W. E. Van, Jucker M. (2006) Mechanism of cerebral beta-amyloid angiopathy: murine and cellular models. Brain Pathol. 16, 40-54.

Herzig M. C., Paganetti P., Staufenbiel M., Jucker M. (2007) BACE1 and mutated presenilin-1 differently modulate Abeta40 and Abeta42 levels and cerebral amyloidosis in APPDutch transgenic mice. Neurodegener. Dis. 4, 127-135.

Herzig M. C., Winkler D. T., Burgermeister P., Pfeifer M., Kohler E., Schmidt S. D., Danner S., et al. (2004) Abeta is targeted to the vasculature in a mouse model of hereditary cerebral hemorrhage with amyloidosis. Nat. Neurosci. 7, 954-960. 
Hirai K., Aliev G., Nunomura A., Fujioka H., Russell R. L., Atwood C. S., Johnson A. B., et al. (2001a) Mitochondrial abnormalities in Alzheimer's disease. J. Neurosci. 21, 30173023.

Hirai K., Aliev G., Nunomura A., Fujioka H., Russell R. L., Atwood C. S., Johnson A. B., et al. (2001b) Mitochondrial abnormalities in Alzheimer's disease. J. Neurosci. 21, 301723.

Hoesen G. Van, Pandya D. N., Butters N. (1975) Some connections of the entorhinal (area 28) and perirhinal (area 35) cortices of the rhesus monkey. II. Frontal lobe afferents. Brain Res. 95, 25-38.

Hyman B. T., Hoesen G. W. Van, Damasio A. R., Barnes C. L. (1984) Alzheimer's disease: cell-specific pathology isolates the hippocampal formation. Science 225, 1168-1170.

Jack C. R. J., Petersen R. C., Xu Y., O’Brien P. C., Smith G. E., Ivnik R. J., Boeve B. F., Tangalos E. G., Kokmen E. (2000) Rates of hippocampal atrophy correlate with change in clinical status in aging and AD. Neurology 55, 484-489.

Jadiya P., Kolmetzky D. W., Tomar D., Meco A. Di, Lombardi A. A., Lambert J. P., Luongo T. S., Ludtmann M. H., Praticò D., Elrod J. W. (2019) Impaired mitochondrial calcium efflux contributes to disease progression in models of Alzheimer's disease. Nat.

Commun. 10, 3885.

Johnson N. A., Jahng G.-H., Weiner M. W., Miller B. L., Chui H. C., Jagust W. J., GornoTempini M. L., Schuff N. (2005) Pattern of cerebral hypoperfusion in Alzheimer disease and mild cognitive impairment measured with arterial spin-labeling MR imaging: initial experience. Radiology 234, 851-859.

Jucker M., Bättig K., Meier-Ruge W. (1990) Effects of aging and vincamine derivatives on pericapillary microenvironment: stereological characterization of the cerebral capillary network. Neurobiol. Aging 11, 39-46.

Kalaria R. N., Hedera P. (1995) Differential degeneration of the cerebral microvasculature in Alzheimer's disease. Neuroreport 6, 477-480.

Kandimalla R., Reddy P. H. (2016) Multiple faces of dynamin-related protein 1 and its role in Alzheimer's disease pathogenesis. Biochim. Biophys. Acta 1862, 814-828. 
Kim Y. J., Kwon H. K., Lee J.-M., Kim Y. J., Kim H. J., Jung N.-Y., Kim S. T., Lee K. H., Na D. L., Seo S. W. (2015) White matter microstructural changes in pure Alzheimer's disease and subcortical vascular dementia. Eur. J. Neurol. 22, 709-716.

Knight E. M., Verkhratsky A., Luckman S. M., Allan S. M., Lawrence C. B. (2012) Hypermetabolism in a triple-transgenic mouse model of Alzheimer's disease. Neurobiol. Aging 33, 187-193.

Kouznetsova E., Klingner M., Sorger D., Sabri O., Großmann U., Steinbach J., Scheunemann M., Schliebs R. (2006) Developmental and amyloid plaque-related changes in cerebral cortical capillaries in transgenic Tg2576 Alzheimer mice, in Int. J. Dev. Neurosci., Vol. 24, pp. 187-193. Int J Dev Neurosci.

Kwan H.-Y., Leung P.-C., Huang Y., Yao X. (2003) Depletion of intracellular Ca2+ stores sensitizes the flow-induced Ca2+ influx in rat endothelial cells. Circ. Res. 92, 286-292.

LaFerla F. M. (2002) Calcium dyshomeostasis and intracellular signalling in alzheimer's disease. Nat. Rev. Neurosci. 3, 862-872.

Lehmann M., Madison C. M., Ghosh P. M., Seeley W. W., Mormino E., Greicius M. D., Gorno-Tempini M. L., et al. (2013) Intrinsic connectivity networks in healthy subjects explain clinical variability in Alzheimer's disease. Proc. Natl. Acad. Sci. U. S. A. 110 , 11606-11611.

Lu M.-H., Zhao X.-Y., Yao P.-P., Xu D.-E., Ma Q.-H. (2018) The Mitochondrion: A Potential Therapeutic Target for Alzheimer's Disease. Neurosci. Bull. 34, 1127-1130.

Luo F., Rustay N. R., Ebert U., Hradil V. P., Cole T. B., Llano D. A., Mudd S. R., Zhang Y., Fox G. B., Day M. (2012) Characterization of 7- and 19-month-old Tg2576 mice using multimodal in vivo imaging: limitations as a translatable model of Alzheimer's disease. Neurobiol. Aging 33, 933-944.

Mancardi G. L., Mandybur T. I., Liwnicz B. H. (1982) Spongiform-like changes in Alzheimer's disease. An ultrastructural study. Acta Neuropathol. 56, 146-150.

Manczak M., Calkins M. J., Reddy P. H. (2011) Impaired mitochondrial dynamics and abnormal interaction of amyloid beta with mitochondrial protein Drp1 in neurons from patients with Alzheimer's disease: implications for neuronal damage. Hum. Mol. Genet. 
20, 2495-2509.

Mann D. M. (1996) Pyramidal nerve cell loss in Alzheimer's disease. Neurodegener. a J. Neurodegener. Disord. neuroprotection, neuroregeneration 5, 423-427.

Masliah E., Mallory M., Deerinck T., DeTeresa R., Lamont S., Miller A., Terry R. D., Carragher B., Ellisman M. (1993) Re-evaluation of the structural organization of neuritic plaques in Alzheimer's disease. J. Neuropathol. Exp. Neurol. 52, 619-632.

Massaad C. A., Amin S. K., Hu L., Mei Y., Klann E., Pautler R. G. (2010) Mitochondrial superoxide contributes to blood flow and axonal transport deficits in the $\mathrm{Tg} 2576$ mouse model of Alzheimer's disease. PLoS One 5.

Maurer I., Zierz S., Möller H. J. (2000) A selective defect of cytochrome c oxidase is present in brain of Alzheimer disease patients. Neurobiol. Aging 21, 455-462.

Melamed E., Lavy S., Bentin S., Cooper G., Rinot Y. (1980) Reduction in regional cerebral blood flow during normal aging in man. Stroke 11, 31-35.

Meyerhoff D. J., MacKay S., Constans J. M., Norman D., Dyke C. Van, Fein G., Weiner M. W. (1994) Axonal injury and membrane alterations in Alzheimer's disease suggested by in vivo proton magnetic resonance spectroscopic imaging. Ann. Neurol. 36, 40-47.

Miao J., Vitek M. P., Xu F., Previti M. Lou, Davis J., Nostrand W. E. Van (2005) Reducing cerebral microvascular amyloid-beta protein deposition diminishes regional neuroinflammation in vasculotropic mutant amyloid precursor protein transgenic mice. J. Neurosci. 25, 6271-6277.

Miyakawa T., Uehara Y., Desaki J., Kimura T., Kuramoto R. (1988) Morphological changes of microvessels in the brain with Alzheimer's disease. Jpn. J. Psychiatry Neurol. 42, 819-824.

Montagne A., Nation D. A., Pa J., Sweeney M. D., Toga A. W., Zlokovic B. V (2016) Brain imaging of neurovascular dysfunction in Alzheimer's disease. Acta Neuropathol. 131, 687-707.

Moreira P. I., Cardoso S. M., Santos M. S., Oliveira C. R. (2006) The key role of mitochondria in Alzheimer's disease. J. Alzheimers. Dis. 9, 101-110. 
Moreira P. I., Carvalho C., Zhu X., Smith M. A., Perry G. (2010) Mitochondrial dysfunction is a trigger of Alzheimer's disease pathophysiology. Biochim Biophys Acta.

Moreira P. I., Duarte A. I., Santos M. S., Rego A. C., Oliveira C. R. (2009) An integrative view of the role of oxidative stress, mitochondria and insulin in Alzheimer's disease. $J$. Alzheimers. Dis. 16, 741-761.

Moreira P. I., Siedlak S. L., Wang X., Santos M. S., Oliveira C. R., Tabaton M., Nunomura A., et al. (2007) Increased autophagic degradation of mitochondria in Alzheimer disease. Autophagy 3, 614-615.

Murray I. V. J., Proza J. F., Sohrabji F., Lawler J. M. (2011) Vascular and metabolic dysfunction in Alzheimer's disease: a review. Exp. Biol. Med. (Maywood). 236, 772782.

Mutisya E. M., Bowling A. C., Beal M. F. (1994) Cortical cytochrome oxidase activity is reduced in Alzheimer's disease. J. Neurochem. 63, 2179-2184.

Niwa K., Porter V. A., Kazama K. E. N., Cornfield D., Carlson G. A., Iadecola C. (2001) A $\beta$ peptides enhance vasoconstriction in cerebral circulation. Am. J. Physiol. - Hear. Circ. Physiol. 281.

Niwa K., Younkin L., Ebeling C., Turner S. K., Westaway D., Younkin S., Ashe K. H., et al. (2000) Abeta 1-40-related reduction in functional hyperemia in mouse neocortex during somatosensory activation. Proc. Natl. Acad. Sci. U. S. A. 97, 9735-40.

Nunomura A., Perry G., Aliev G., Hirai K., Takeda A., Balraj E. K., Jones P. K., et al. (2001) Oxidative damage is the earliest event in Alzheimer disease. J. Neuropathol. Exp. Neurol. 60, 759-767.

Oddo S., Caccamo A., Kitazawa M., Tseng B. P., LaFerla F. M. (2003a) Amyloid deposition precedes tangle formation in a triple transgenic model of Alzheimer's disease. Neurobiol. Aging 24, 1063-1070.

Oddo S., Caccamo A., Shepherd J. D., Murphy M. P., Golde T. E., Kayed R., Metherate R., Mattson M. P., Akbari Y., LaFerla F. M. (2003b) Triple-transgenic model of Alzheimer's disease with plaques and tangles: intracellular Abeta and synaptic dysfunction. Neuron 39, 409-421. 
Park L., Zhou P., Koizumi K., Jamal S. El, Previti M. Lou, Nostrand W. E. Van, Carlson G., Iadecola C. (2013) Brain and circulating levels of A $\beta 1-40$ differentially contribute to vasomotor dysfunction in the mouse brain. Stroke 44, 198-204.

Parker W. D. J., Filley C. M., Parks J. K. (1990) Cytochrome oxidase deficiency in Alzheimer's disease. Neurology 40, 1302-1303.

Poisnel G., Hérard A. S., Tannir El Tayara N. El, Bourrin E., Volk A., Kober F., Delatour B., et al. (2012) Increased regional cerebral glucose uptake in an APP/PS1 model of Alzheimer's disease. Neurobiol. Aging 33, 1995-2005.

Rui Y., Tiwari P., Xie Z., Zheng J. Q. (2006) Acute impairment of mitochondrial trafficking by beta-amyloid peptides in hippocampal neurons. J. Neurosci. 26, 10480-10487.

Rui Y., Zheng J. Q. (2016) Amyloid $\beta$ oligomers elicit mitochondrial transport defects and fragmentation in a time-dependent and pathway-specific manner. Mol. Brain 9, 79.

Sabuncu M. R., Desikan R. S., Sepulcre J., Yeo B. T. T., Liu H., Schmansky N. J., Reuter M., et al. (2011) The dynamics of cortical and hippocampal atrophy in Alzheimer disease. Arch. Neurol. 68, 1040-1048.

Sagare A. P., Bell R. D., Zlokovic B. V (2013) Neurovascular defects and faulty amyloid- $\beta$ vascular clearance in Alzheimer's disease. J. Alzheimers. Dis. 33 Suppl 1, S87-100.

Schmitz C., Rutten B. P. F., Pielen A., Schäfer S., Wirths O., Tremp G., Czech C., et al. (2004) Hippocampal Neuron Loss Exceeds Amyloid Plaque Load in a Transgenic Mouse Model of Alzheimer's Disease. Am. J. Pathol. 164, 1495-1502.

Selkoe D. J., Hardy J. (2016) The amyloid hypothesis of Alzheimer's disease at 25 years. EMBO Mol. Med. 8, 595-608.

Shibata M., Yamada S., Kumar S. R., Calero M., Bading J., Frangione B., Holtzman D. M., et al. (2000) Clearance of Alzheimer's amyloid-ss(1-40) peptide from brain by LDL receptor-related protein-1 at the blood-brain barrier. J. Clin. Invest. 106, 1489-1499.

Shin H. K., Jones P. B., Garcia-Alloza M., Borrelli L., Greenberg S. M., Bacskai B. J., Frosch M. P., Hyman B. T., Moskowitz M. A., Ayata C. (2007) Age-dependent cerebrovascular dysfunction in a transgenic mouse model of cerebral amyloid 
angiopathy. Brain 130, 2310-2319.

Silva D. F., Selfridge J. E., Lu J., E L., Cardoso S. M., Swerdlow R. H. (2012) Mitochondrial abnormalities in Alzheimer's disease: possible targets for therapeutic intervention. Adv. Pharmacol. 64, 83-126.

Smith T. W., Anwer U., DeGirolami U., Drachman D. A. (1987) Vacuolar change in Alzheimer's disease. Arch. Neurol. 44, 1225-1228.

Snellman A., Takkinen J. S., López-Picón F. R., Eskola O., Solin O., Rinne J. O., Haaparanta-Solin M. (2019) Effect of genotype and age on cerebral [(18)F]FDG uptake varies between transgenic APP(Swe)-PS1(dE9) and Tg2576 mouse models of Alzheimer's disease. Sci. Rep. 9, 5700.

Stoub T. R., deToledo-Morrell L., Stebbins G. T., Leurgans S., Bennett D. A., Shah R. C. (2006) Hippocampal disconnection contributes to memory dysfunction in individuals at risk for Alzheimer's disease. Proc. Natl. Acad. Sci. U. S. A. 103, 10041-10045.

Su B., Wang X., Nunomura A., Moreira P. I., Lee H., Perry G., Smith M. A., Zhu X. (2008) Oxidative stress signaling in Alzheimer's disease. Curr. Alzheimer Res. 5, 525-532.

Swerdlow R. H., Burns J. M., Khan S. M. (2014) The Alzheimer's disease mitochondrial cascade hypothesis: progress and perspectives. Biochim. Biophys. Acta 1842, 12191231.

Swerdlow R. H., Khan S. M. (2009) The Alzheimer's disease mitochondrial cascade hypothesis: an update. Exp. Neurol. 218, 308-315.

Taarnhøj N. C. B. B., Munch I. C., Sander B., Kessel L., Hougaard J. L., Kyvik K., Sørensen T. I. A., Larsen M. (2008) Straight versus tortuous retinal arteries in relation to blood pressure and genetics. Br. J. Ophthalmol. 92, 1055-1060.

Takeda S., Sato N., Takeuchi D., Kurinami H., Shinohara M., Niisato K., Kano M., Ogihara T., Rakugi H., Morishita R. (2009) Angiotensin receptor blocker prevented $\beta$-amyloidinduced cognitive impairment associated with recovery of neurovascular coupling. Hypertension 54, 1345-1352.

Tannir El Tayara N. El, Delatour B., Volk A., Dhenain M. (2010) Detection of vascular 
alterations by in vivo magnetic resonance angiography and histology in APP/PS1 mouse model of Alzheimer's disease. Magn. Reson. Mater. Physics, Biol. Med. 23, 53-64.

Tayara N. E. T. El, Volk A., Dhenain M., Delatour B. (2007) Transverse relaxation time reflects brain amyloidosis in young APP/PS1 transgenic mice. Magn. Reson. Med. 58, $179-184$.

Terry R. D. (1996) The pathogenesis of Alzheimer disease: an alternative to the amyloid hypothesis. J. Neuropathol. Exp. Neurol. 55, 1023-1025.

Thomas T., Miners S., Love S. (2015) Post-mortem assessment of hypoperfusion of cerebral cortex in Alzheimer's disease and vascular dementia. Brain 138, 1059-1069.

Tomimoto H., Akiguchi I., Wakita H., Suenaga T., Nakamura S., Kimura J. (1997) Regressive changes of astroglia in white matter lesions in cerebrovascular disease and Alzheimer's disease patients. Acta Neuropathol. 94, 146-152.

Verma M., Howard R. J. (2012) Semantic memory and language dysfunction in early Alzheimer's disease: a review. Int. J. Geriatr. Psychiatry 27, 1209-1217.

Vinters H. V, Secor D. L., Read S. L., Frazee J. G., Tomiyasu U., Stanley T. M., Ferreiro J. A., Akers M. A. (1994) Microvasculature in brain biopsy specimens from patients with Alzheimer's disease: an immunohistochemical and ultrastructural study. Ultrastruct. Pathol. 18, 333-348.

Walsh D. M., Selkoe D. J. (2007) A beta oligomers - a decade of discovery. J. Neurochem. 101, 1172-1184.

Weidensteiner C., Metzger F., Bruns A., Bohrmann B., Kuennecke B., Kienlin M. Von (2009) Cortical hypoperfusion in the B6.PS2APP mouse model for Alzheimer's disease: Comprehensive phenotyping of vascular and tissular parameters by MRI. Magn. Reson. Med. 62, 35-45.

Xie H., Guan J., Borrelli L. A., Xu J., Serrano-Pozo A., Bacskai B. J. (2013) Mitochondrial alterations near amyloid plaques in an Alzheimer's disease mouse model. J. Neurosci. 33, 17042-51.

Xu F., Grande A. M., Robinson J. K., Previti M. L., Vasek M., Davis J., Nostrand W. E. Van 
(2007) Early-onset subicular microvascular amyloid and neuroinflammation correlate with behavioral deficits in vasculotropic mutant amyloid beta-protein precursor transgenic mice. Neuroscience 146, 98-107.

Yamamoto K., Korenaga R., Kamiya A., Qi Z., Sokabe M., Ando J. (2000) P2X(4) receptors mediate ATP-induced calcium influx in human vascular endothelial cells. Am. $J$. Physiol. Heart Circ. Physiol. 279, H285-92.

Yin R., Lu C., Chen Q., Fan J., Lu J. (2013) Microvascular damage is involved in the pathogenesis of heroin induced spongiform leukoencephalopathy. Int. J. Med. Sci. 10, 299-306.

Zabel M., Schrag M., Crofton A., Tung S., Beaufond P., Ornam J. Van, Dininni A., Vinters H. V, Coppola G., Kirsch W. M. (2013) A shift in microglial $\beta$-amyloid binding in Alzheimer's disease is associated with cerebral amyloid angiopathy. Brain Pathol. 23, $390-401$.

Zand L., Ryu J. K., McLarnon J. G. (2005) Induction of angiogenesis in the $\beta$-amyloid peptide-injected rat hippocampus. Neuroreport 16, 129-132.

Zhang X., Fu Z., Meng L., He M., Zhang Z. (2018) The Early Events That Initiate $\beta$ Amyloid Aggregation in Alzheimer's Disease. Front. Aging Neurosci. 10.

Zlokovic B. V (2008) New therapeutic targets in the neurovascular pathway in Alzheimer's disease. Neurotherapeutics 5, 409-414.

Zola-Morgan S., Squire L. R., Amaral D. G. (1986) Human amnesia and the medial temporal region: enduring memory impairment following a bilateral lesion limited to field CA1 of the hippocampus. J. Neurosci. 6, 2950-2967. 